Immune responses in neonatal and adult plos following Joll-like receptor agonist| adjuvanted vaccination

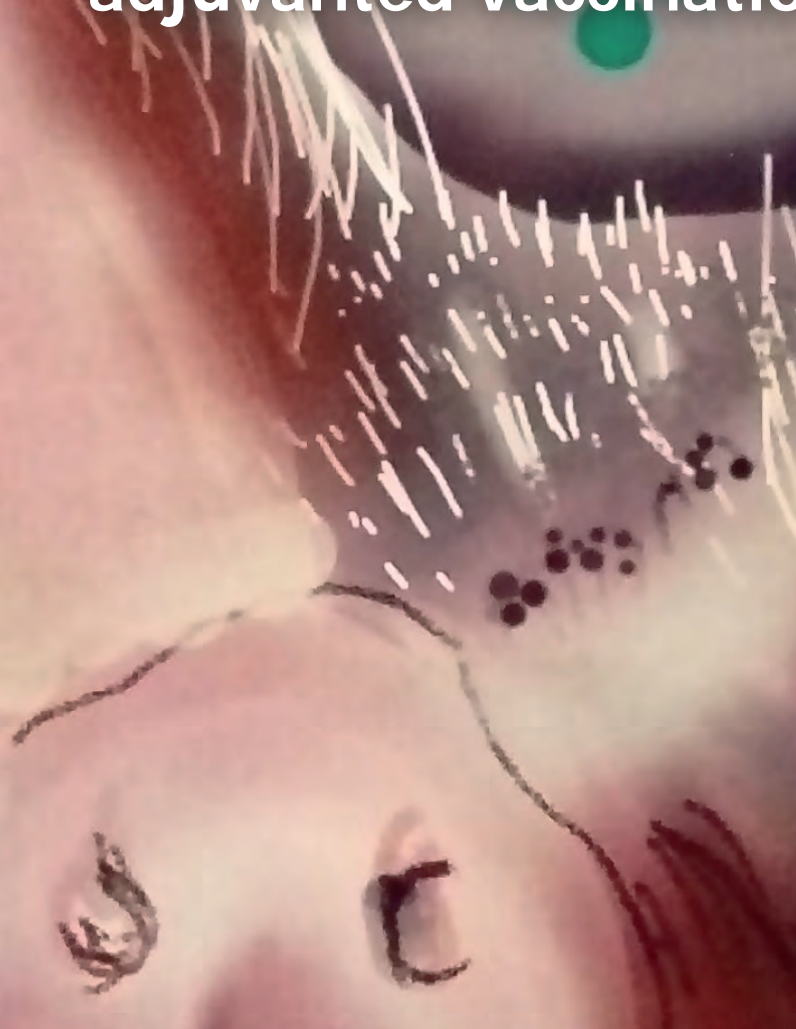




\section{Propositions}

1. Toll-like receptor $7 / 8$ agonists are high potentials for neonatal vaccines (this thesis).

2. Regarding the number of immune-stimulators in an adjuvant formulation, less can be more (this thesis).

3. Considering plasmacytoid dendritic cells as professional antigen presenting cells is questionable.

4. The final group size is determined by careful statistics and random fate.

5. Histopathologic analysis is critical to validate the relevance of detected pathogens.

6. Only in politics, in vitro research can replace in vivo research.

7. Vaccination is a sustainable method to reduce disease risk, but is not meant as a tool to intensify livestock farming.

8. Pigs are of major importance for human health, although most people only value their spare ribs.

Propositions belonging to the thesis, entitled:

‘ Immune responses in neonatal and adult pigs following Toll-like receptor agonist adjuvanted vaccination'

Sandra Vreman

Wageningen, $7^{\text {th }}$ of October, 2020 

Immune responses in neonatal and adult pigs

following Toll-like receptor agonist

adjuvanted vaccination

Sandra Vreman 


\section{Thesis committee}

\section{Promotor}

Prof. Dr H.F.J. Savelkoul

Personal chair, Cell Biology and Immunology group

Wageningen University \& Research

\section{Co-promotors}

Prof. Dr J.M.J. Rebel

Special professor, Healthy and Resilient Livestock

Wageningen University \& Research

Dr N. Stockhofe-Zurwieden

Researcher, Infection Biology

Wageningen University \& Research

\section{Other members}

Prof. Dr W.M.H. van der Poel, Wageningen University \& Research

Prof. Dr A. Gröne, University of Utrecht

Prof. Dr V.P.M.G. Rutten, University of Utrecht

Dr I. Schwartz-Cornil, French National Institute for Agriculture, Food, and Environment, Jouy-en-Josas, France

This research was conducted under the auspices of the Graduate School Wageningen Institute of Animal Sciences 


\title{
Immune responses in neonatal and adult pigs following Toll-like receptor agonist adjuvanted vaccination
}

\author{
Sandra Vreman
}

Thesis

submitted in fulfilment of the requirements for the degree of doctor at Wageningen University

by the authority of the Rector Magnificus,

Prof. Dr A.P.J. Mol, in the presence of the

Thesis Committee appointed by the Academic Board to be defended in public on Wednesday 7 October 2020 at 4 p.m. in the Aula. 


\section{Sandra Vreman}

Immune responses in neonatal and adult pigs following Toll-like receptor agonist adjuvanted vaccination, 206 pages

PhD thesis, Wageningen University, Wageningen, the Netherlands (2020)

With references, with summaries in Dutch and English

ISBN: 978-94-6395-367-2

https://doi.org/10.18174/518637 
Voor mamma en pappa 
People who look for the first time through a microscope say now I see this and then I see thatand even a skilled observer can be fooled. On these observations I have spent more time than many will believe, but I have done them with joy, and I have taken no notice of those who have said why take so much trouble and what good is it?

Antonie van Leeuwenhoek 


\section{Table of contents}

$\begin{array}{lll}\text { Chapter } 1 \text { General introduction } & 9\end{array}$

Chapter 2 Neonatal porcine blood derived dendritic cell subsets show activation after TLR2 or TLR9 stimulation

Chapter 3 Early immune responses in skin and lymph node after skin vaccination in neonatal and adult pigs

Chapter 4 Toll-Like receptor agonists as adjuvants for inactivated porcine reproductive and respiratory syndrome virus (PRRSV) vaccine

Chapter 5 Immune responses induced by inactivated porcine reproductive and respiratory syndrome Virus (PRRSV) vaccine in neonatal pigs using different adjuvants

Chapter 6 General discussion

Appendix Abbreviations 182

Summary 185

Samenvatting 188

About the author 194

Dankwoord 200 
General introduction 



\section{Introduction}

Early-life is a critical period in the development of piglets towards a more mature immune status and is a phase in life with a high risk of encountering infectious agents. In swine farms, infections of young piglets often causes diseases that can result in serious health and welfare problems leading to severe economic losses worldwide ${ }^{1-4}$. To protect against these infections, vaccination is widely considered to be one of the most powerful strategies ${ }^{5}$. However, newborns are known to respond differently to vaccines than adults (see section on neonatal immune responses), which complicates vaccine development for neonatal pigs. In this thesis, we study potent adjuvants (see section on vaccine formulation and delivery), such as synthetic Toll-like receptor (TLR) agonists, and a novel delivery system for skin vaccination in adult or immunocompetent ( $>$ 8-week-old) and neonatal or newborn ( $<1$ - week-old) pigs, as means to enhance the immune response after vaccination, and to investigate to necessity of age-dependent vaccine formulations.

\section{Professional cells for vaccine-antigen presentation: dendritic cells}

In the immune system, several cell types have the ability to present vaccine-antigen to naïve T-cells. These cells are named antigen-presenting cells (APCs). APCs include cell such as dendritic cells (DCs), macrophages and B-cells. Among those, DCs are the most potent $A P C s^{6}$. The various DC subsets form a heterogenous group of cells that share their capacity for highly efficient presentation of antigens to naive T-cells. Thus, DCs are an important link between the innate and adaptive immune system ${ }^{7}$. DCs reside predominantly in an immature state in a wide variety of tissues and in blood. They are especially abundant near mucosal surfaces and in the skin, where their dendrites are easily exposed to different endogenous and exogeneous antigens or pathogens. All DCs display various pattern recognition receptors (PRR) that are required to detect and control pathogens. DCs can possess different PRR types such as Toll-like receptors (TLRs), NOD-like receptors (NLRs), RIG-like helicases (RLHs), and C-type lectin-like receptors (CLRs) ${ }^{8}$. After recognition of the antigen by the PRR and endocytosis of the antigen by the DC, DCs get activated and migrate to the draining lymph node to present the antigen to naive-T-cells. Cytokines such as TNF, IL-1 and IL-12 can stimulate this migration ${ }^{6,9}$. In order to activate naïve-T-cells the cell-surface of migrated and mature DCs needs to contain major histocompatibility complex (MHC) II and co-stimulatory molecules such as CD40 and CD80/86 ${ }^{10}$. Compared to mature DCs, immature DCs express only a limited amount of $\mathrm{MHCll}$ and co-stimulatory molecules on their cell-surfaces, but show more endocytic potential compared to mature $\mathrm{DCs}{ }^{11}$.

\section{Blood derived DCs and monocyte derived DCs}

The different DC subsets, their activation and subsequent maturation have been extensively studied and reviewed in mice and humans ${ }^{12,13}$, and also more recently in pigs ${ }^{14,15}$. Most researchers have used blood-derived monocytes or bone marrow cells cultured 
in vitro with GM-CSF and/or IL-4 ${ }^{16,17}$, thus creating monocyte derived DCs (MoDCs) or bone marrow derived DCs (BMDCs), respectively. These cultured DCs are relatively easy to generate in sufficient numbers. However, the MoDCs in particular do not fully represent the steady-state/resident DCs, but are more related to an inflammatory type DC, which is derived from extravasated monocytes ${ }^{18}$. The typical steady-state $D C$ that is found in tissues or in blood originates from bone marrow DC precursors and are dependent on the growth factor fms-like tyrosine kinase 3 ligand (FLT3L) and its receptor FLT3 ${ }^{19}$.

To identify and investigate the competence and capacity of the different steady-state $D C$ subsets in vitro, blood derived $D C s$ (BDCs) are a widely used alternative for MoDCs and BMDCs. BDCs can be identified within peripheral blood mononuclear cells (PBMCs) isolated from whole blood using flow cytometry to identify specific surface-markers. However, BDCs are more difficult to obtain and to study because of their limited number in the PBMC population ${ }^{20}$. These BDCs consist of different subsets, which have recently also been identified in pigs: plasmacytoid DCs (pDCs) and conventional DCs (cDCs), which can be further divided into a $\mathrm{CDC} 1$ and a $\mathrm{CDC} 2$ subset ${ }^{21-23}$. $\mathrm{CDCs}$ are most efficient at presenting antigen and activating naïve T-cells ${ }^{24}$, while pDCs are especially important for anti-viral responses through production of high amounts of type I interferon (IFN- $\alpha / \beta)$ and other cytokines, such as TNF and IL-12 ${ }^{15,22}$.

Because BDCs are closely related to the steady-state DCs, and are more age-specific, due to no additional culture being needed, we used BDCs in chapter 2 . In this in vitro experiment, we applied different surface-markers (Table 1) to isolated PBMCs to study the pDCs, cDC1 and cDC2 subsets in blood of adult and neonatal pigs.

Table 1: Overview cell-surface-markers to identify blood derived dendritic cell (BDC) subsets.

\begin{tabular}{lllll}
\hline & \multicolumn{2}{l}{ Blood derived DC } & \multicolumn{2}{l}{ Description of the marker } \\
\hline Surface-marker & pDC & CDC1 & CDC2 & \\
\hline CD4 & + & - & - & $\begin{array}{l}\text { Co-receptor of T-cell receptor to assists the interaction } \\
\text { with APC }\end{array}$ \\
\hline CD14 & - & - & - & $\begin{array}{l}\text { Co-receptor with TLR2 and MD2 for detection of } \\
\text { bacterial LPS }\end{array}$ \\
\hline CD172a & + & low & high & SIRPa, which binds to CD47 (phagocytosis) 25 \\
\hline CADM-1 & - & + & + & Cell adhesion molecule 1, organization of cell junction \\
\hline CD80/86 & $-/ l o w$ & + & + & $\begin{array}{l}\text { Binds to CD28 on T-cells, co-stimulatory signal for T-cell } \\
\text { activation }\end{array}$ \\
\hline MHCII & $-/ l o w$ & + & + & $\begin{array}{l}\text { Presenting antigens from extracellular proteins after } \\
\text { endocytosis }\end{array}$ \\
\hline
\end{tabular}

Different surface-markers were used to identify three different DC subsets of BDCs (plamacytoid $\mathrm{DC}(\mathrm{pDC})$ and conventional $\mathrm{DC}(\mathrm{CDC} 1$ and $\mathrm{CDC} 2))$ in porcine peripheral blood mononuclear cells (PBMCs) ${ }^{21,22}$. Abbreviations: cluster of differentiation (CD); antigen presenting cell (APC); Toll-like receptor (TLR); lipopolysaccharide (LPS); lymphocyte antigen 96 (MD2); signal regulatory protein a (SIRPa) major histocompatibility complex (MHC) II. 


\section{Skin DCs}

The skin forms a protective barrier against exposure to pathogens and other environmental influences, and contains a large number of APCs, closely connected to draining lymph vessels (Figure 1). This makes the skin an attractive organ for vaccine delivery ${ }^{26}$ in which skin DCs play an essential role in presenting the vaccine-antigen to naive-T-cells. The outer avascular epithelial layer of the skin (epidermis) harbours a special subset of DCs, the Langerhans cells (LCs) that express Langerin (CD207). LCs originate in the fetal liver and are not derived from a bone marrow precursor as the previously described DC subsets. They proliferate by self-renewal in quiescent skin, or after skin inflammation new LCs may derive from monocytes or precursors in the bone marrow ${ }^{27,28}$.

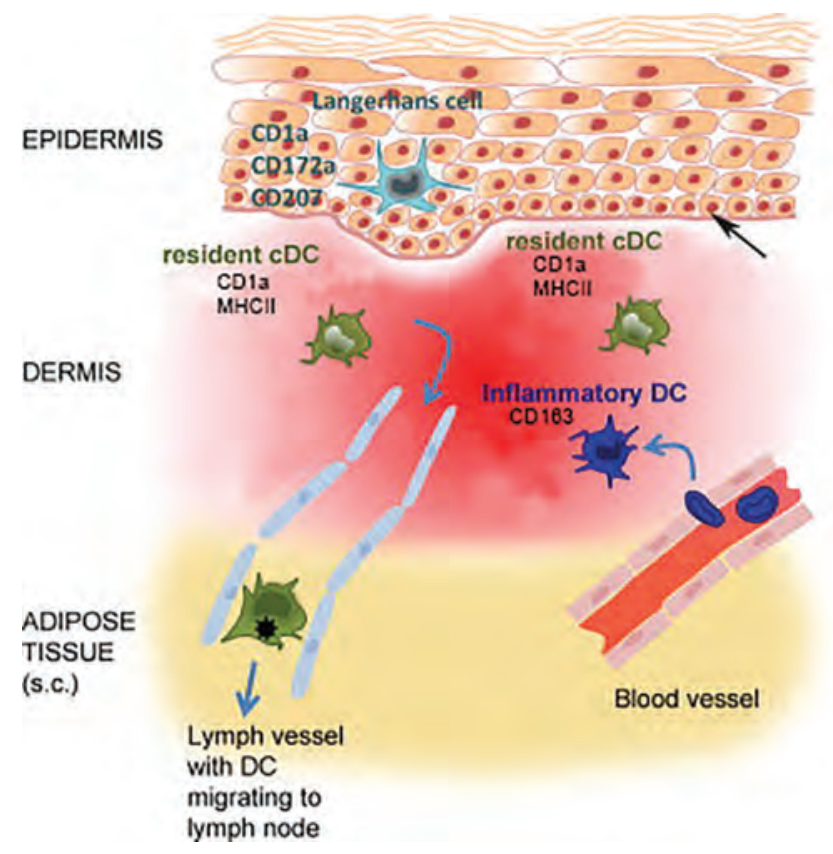

Figure 1: Antigen presenting cells (APCs) in different layer of the porcine skin.

The most important markers to identify different APCs in the skin are presented. The following APCs are pictured: Langerhans cell (LC), resident dermal dendritic cells (dDC), inflammatory dDC (MoDC) and dermal macrophages (M $\phi)$ (adapted from Summerfield et al. ${ }^{29}$, arrow points to the basement membrane.

The dermis of the skin, which is mainly composed of type I collagen and elastin fibers, is firmly attached by a basement membrane to the epidermis, and contains dermal DCs (dDCs). Human and mice dDCs have been extensively reviewed ${ }^{30}$ and a large number of markers has been tested to identify the different dDCs subsets and their role in the 
induction and progression of the immune response. In chapter 3 we used the surfacemarkers CD1a, CD163 and MHCII to identify and quantify different APCs in situ in the skin of adult and neonatal pigs with immunofluorescence (IF) microscopy based on research in human ${ }^{27,31}$ and porcine ${ }^{32}$ skin (Table 2). With these surface markers we identified, in addition to the epidermal LCs, two different dermal DC subsets: resident dDCs (dDCs) and inflammatory dDCs (MoDCs), and dermal macrophages (M $\phi)$. The different skin APCs could be further specified based on their origin: bone marrow precursor, monocytederived or fetal origin.

Table 2: Specification of antigen presenting cell (APC) populations within porcine skin.

\begin{tabular}{llllll}
\hline & \multicolumn{2}{l}{ Skin APC } & & Description of the marker and references \\
\hline Location skin & E & D & D & D & Epidermis (E) or dermis (D) \\
\hline Surface marker & & & & & \\
\hline CD1a & + & + & $+/-$ & - & Mediate presentation of antigen to T-cells ${ }^{29}$ \\
\hline CD163 & - & $-/ l o w$ & + & + & Scavenger receptor ${ }^{33}$ \\
\hline MHCII & + & + & + & - & $\begin{array}{l}\text { Presenting antigens from extracellular proteins } \\
\text { after endocytosis }{ }^{29,32}\end{array}$ \\
\hline Origin & FL & BM & Mo & BM/Mo & $\begin{array}{l}\text { Derived from bone marrow precursor (BM), } \\
\text { monocytes (Mo) or fetal liver (FL) }\end{array}$ \\
\hline
\end{tabular}

Different subsets of APCs (Langerhans cell (LC), dermal dendritic cell (dDC), monocyte derived DC (MoDC) and dermal macrophage $(M \phi)$ ) were analysed for location in skin, expression of surfacemarkers and origin. Abbreviations: cluster of differentiation (CD), major histocompatibility complex (MHC) II.

\section{Vaccine-specific immune responses}

Both the innate and the adaptive immune system are required to induce an effective vaccine response. The innate immune system consists of cells (neutrophils, dendritic cells (DCs), macrophages and natural killer (NK) cells), and soluble molecules like cytokines, chemokines, acute phase proteins, and the complement system ${ }^{34}$. This system reacts quickly, but lacks specificity and is not equipped to induce a vaccine-specific long-lasting immunological memory. It is therefore not a suitable target to induce a vaccine-related protective immune response. Rapid antigen detection by APCs followed by endocytosis and antigen delivery, are important pre-conditions to shape an adaptive immune response ${ }^{7,35}$. The adaptive immune system is highly antigen-specific and uses precise antigen-specific receptors on T-cells and B-cells. Although, the successful formation of an antigen-specific memory is much slower in onset (days to weeks), it is this antigen-specific response which is the basis of vaccination. 
Before a vaccine is able to produce this immunologic memory, naive CD4+ ${ }^{+}$-cells are transformed into $\mathrm{CD}^{+} \mathrm{T}$ helper (Th) cells or effector $\mathrm{CD} 4^{+} \mathrm{T}$-cells. This process is mediated by different cytokine profiles produced by APCs and other innate cells in the microenvironment, under the direction of different subsets of Th cells ${ }^{36}$. Different Th celldirected responses have been characterized in the context of vaccine efficacy. The Th1induced cell-mediated response is especially important for vaccines against intracellular and viral infections, and is characterized by activation of cytotoxic T-cells and macrophages under the influence of polarizing cytokines IL-12, IFN- $\alpha / \beta$ and IFN- - . The Th2-response is characterized by the production of polarizing cytokines IL-4, IL-5 and IL-13, and supports the production of antigen-specific antibodies ${ }^{37}$. Next to the classical Th1/Th2 response, other effector CD4+ ${ }^{+}$-cells such as Th17 cells (enhanced neutrophil response and a subsequent inflammatory response), $T$ follicular helper cells $\left(T_{F H}\right)$ (activation of B-cell maturation) and $T$ regulatory cells ( $T_{\text {REG }}$ ) (suppressing the proliferation and function of other Th-cells) and their related cytokines can also contribute to effective vaccine responses ${ }^{38}$. In summary, DC activation/migration, Th cell polarizing cytokines, the formation of memory $T$ and Bcells all are essential for effective vaccine responses.

\section{Neonatal immune responses}

In general, neonatal immune systems are less responsive to vaccination compared to adults due to distinct differences in their innate and adaptive immune system, which have been extensively reviewed in humans ${ }^{39,40}$. Fundamental research on porcine neonatal responses to vaccines is limited. However, because the porcine immune system is, in general, similar to the human immune system ${ }^{41,42}$, discussions here about porcine neonatal vaccine responses are based on human research and further substantiated where available with porcine studies.

\section{Neonatal T helper cell responses limit adaptive vaccine responses}

The overall immune response in newborns is skewed towards a Th2-response, most likely to avoid the in utero Th1-mediated alloimmune reactions between mother and foetus, and also to allow microbial colonisation without inducing an inflammatory response ${ }^{43}$. High levels of Th2-directed cytokines (IL-4 and IL-13) are easily generated by neonatal immune cells, while the production of Th1 cytokines (IL-12, IFN- $\alpha / \beta$ and IFN- $\gamma$ ) is impaired ${ }^{44,45}$. Moreover, the high concentrations of adenosine in human neonatal plasma have been shown to inhibit production of Th1-polarizing cytokines ${ }^{46}$. More recently, the influence of other Th subsets on the neonatal immune response have come to attention. Neonatal immune cells produce more Th17-directed pro-inflammatory cytokines (IL-6 and IL-23) compared to adults ${ }^{44,47}$ and this pro-inflammatory condition could be beneficial for protection against microbial infections ${ }^{48,49}$, although an exaggerated inflammation could also lead to pathological conditions. Additionally, there is an increase in suppressive $\mathrm{T}_{\mathrm{REG}}$ cells and IL-10 production, which enhances the induction of tolerance of APCs and 
limits other effector Th-cell responses ${ }^{47}$. Altogether, neonatal Th cell responses hamper the protection against intracellular pathogens and the induction of effective vaccine responses (Figure 2).

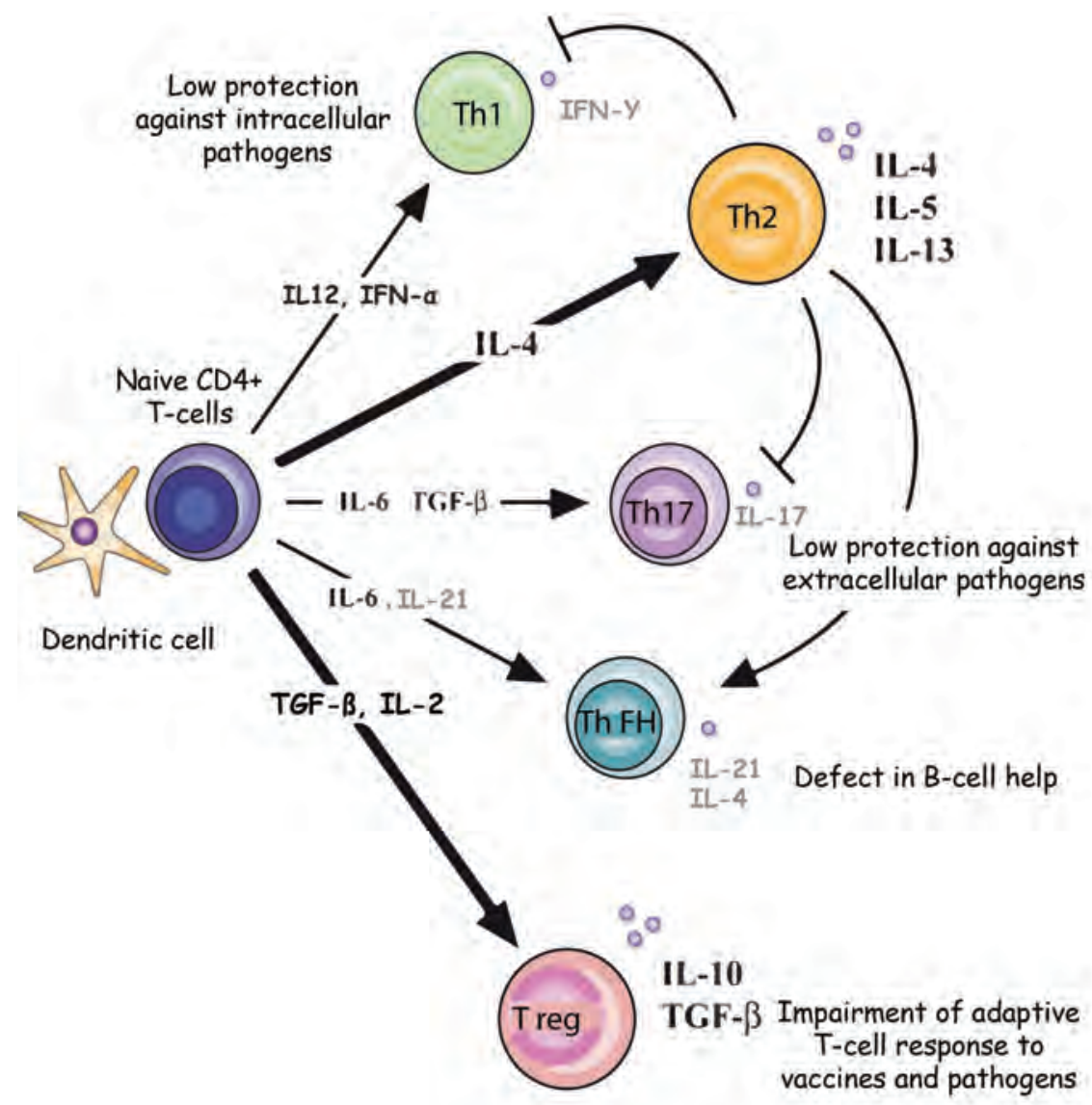

Figure 2: $\mathrm{CD4}^{+} \mathrm{T}$-cell differentiation in human neonates.

Enhanced Th2 and $\mathrm{T}_{\mathrm{REG}}$ differentiation and restricted $\mathrm{Th} 1$ and $\mathrm{T}_{\mathrm{FH}}$ impairs the adaptive $\mathrm{T}$-cell responses after vaccination (adapted from Debock et al. ${ }^{47}$ ).

\section{Neonates develop lower levels of specific antibodies}

The humoral immune response is initiated differently in human ${ }^{39}$ and porcine ${ }^{50}$ neonates. Newborns develop a lower and less constant specific antibody response compared to their immunocompetent adult counterparts after vaccination. Several mechanisms contribute to this decreased neonatal B-cell response, as reviewed by Siegrist et al. ${ }^{51}$. They conclude that neonates primarily develop memory B-cells instead of the antibody-producing 
plasma cells after antigen exposure, and that $\mathrm{T}_{\mathrm{FH}}$ are the key factor for this altered balance 52 (Figure 2). Neonatal pigs have an immature B-cell population with a high percentage of un-primed B-cells $\left(C D 2{ }^{+} C D 21^{+}\right)$. This $C D 2^{+}$expression is down-regulated as the pigs get older ${ }^{53-55}$ and are exposed to an intestinal microflora, inducing primed B-cells (CD2 $\mathrm{CD}^{2} 1^{+}$). It is possible that the higher percentage of primed B-cells in adult pigs, which could be more prone to switch to antibody producing plasma cells, contributes to a more effective humoral immune response in adults compared to neonates.

\section{Antigen presenting properties of neonatal DCs could be less developed}

Priming of the adaptive immune response in neonates could be complicated by impaired functioning of APCs ${ }^{56,57}$. Studies on human umbilical-cord blood and mice splenocytes have suggested functional deficits in neonatal DCs, because of their lower induced expression of co-stimulatory molecules CD80/86 and lesser expression of $\mathrm{MHCll}$, combined with a reduced production of Th1 polarizing cytokines. In addition, in neonatal cord blood the $\mathrm{pDC}-\mathrm{cDC}$ ratio $(3: 1)$ is reversed compared to adults $(1: 3)^{58}$. This could indicate that the neonatal DC population is less antigen-presenting orientated, as CDCs are mainly involved in presentation of antigens. In contrast, the limited research focused on neonatal porcine DCs, showed that there was no difference in the percentage of pDCs in neonatal and adult PBMC population isolated from whole blood ${ }^{21}$. However, the pDC-cDC ratio was not investigated in this porcine study. Moreover, the same study found no differences between neonates and adults in the expression of CD80/86 on BDCs, suggesting that the maturation status of porcine DCs of both age groups is most likely at an equal level.

As porcine skin shows a high degree of similarity with human skin ${ }^{29,59}$, we restricted ourselves to the available literature describing skin DCs in human and porcine neonates. Human neonatal skin harbours a comparable number of DCs as adult skin ${ }^{60}$ and neonatal LCs already show an 'adult-like' phenotype ${ }^{61}$ around birth. Unfortunately, only limited information is available about the migratory capacity of the neonatal DCs towards draining lymph nodes. The normal, unstimulated human neonatal epidermis, contains a higher level of IL-1 $\beta$ and a lower level of TNF compared to adults ${ }^{62}$. As both cytokines are involved in DC migration ${ }^{10}$, this could potentially influence the migration of DCs after skin vaccination. To our knowledge, the quantitative and qualitative properties of porcine DCs in neonatal skin have not been studied previously and will be investigated in chapter $\mathbf{3}$.

\section{Vaccine formulation and delivery}

\section{Vaccine antigen: inactivated porcine reproductive and respiratory syndrome virus (iPRRSV)}

Porcine reproductive and respiratory syndrome virus (PRRSV) is one of the most important worldwide porcine pathogens, which causes abortions in sows, respiratory diseases, and increased susceptibility to other infections in nursery and fattening pigs ${ }^{63}$. PRRSV is an enveloped single-stranded RNA virus (family Arteriviridae) and pigs are its 
only natural host. Two distinct species of PRRSV are recognized: PRRSV-type 1 (European origin, PRRSV-1) and PRRSV-type 2 (North American origin, PRRSV-2) ${ }^{64}$. The interaction of PRRSV with the immune system has been extensively reviewed ${ }^{65-67}$, but the application of different study methods, different PRRSV types/strains, and different pig breeds can lead to ambiguity in interpreting the results. On the other hand, the basic concepts of PRRSVrelated immunology are widely recognized. The main target-cells for PRRSV are cells of the monocyte/macrophage lineage, which need to express CD163 ${ }^{68}$ for virus internalization. Macrophages from genome-edited pigs lacking CD163 are fully resistant to both PRRSV types while maintaining their biological function ${ }^{69}$. PRRSV-infected macrophages produce less IFN- $a$ and are polarized to a state considered less efficient in viral clearance ${ }^{70}$. Moreover, decreased NK-cell function and increased anti-inflammatory IL-10 production induce an overall meagre innate immune response, resulting in the delayed development of an adaptive immune response. These characteristics result in a poor response to the PRRSV-antigen in vaccines and emphasizes the need for highly immuno-stimulatory adjuvants, especially when the antigen is inactivated which further weakens its immunostimulatory effect.

Besides the interaction PRRSV with the immune system, the high mutation rate of the virus ${ }^{71}$ is also a challenge for PRRSV vaccine development. Both hamper the protective immune responses ${ }^{68}$ after vaccination. Live-or attenuated PRRSV vaccines are the most effective vaccines at the moment, but they can also induce viral shedding or mutation of the viral antigen (as occurred in Denmark in $2019{ }^{72}$ ), and are therefore considered less safe ${ }^{73}$. In this thesis, we aimed to increase the potency of inactivated vaccines for PRRSV. The inactivated (i)- PRRSV-antigen selected for the vaccination studies (PRRSV 07 V063) in this research has already been used successfully in adult pigs and has showed enhanced immunogenic properties compared to a commercial iPRRSV vaccine ${ }^{74,75}$. Throughout this thesis, we used the iPRRSV $07 \mathrm{~V} 063$ antigen combined with various adjuvant combinations in all the vaccines.

\section{Adjuvant selection}

Adjuvants are vaccine compounds that enhance the host vaccine-specific immune response, without interfering with it. Thus, the selection of an appropriate immunostimulator or adjuvant is of major importance, especially when combined with a weak/inactivated antigen ${ }^{76}$ like the iPRRSV. An effective adjuvant can enhance the immunogenicity of the vaccine antigen by eliciting a pro-inflammatory environment at the injection site via recruitment and maturation of APCs (macrophages and DCs), induction of the cytokine production, enhancing of the maturation of DCs, all of which improve vaccine antigen presentation ${ }^{77}$. Moreover, adjuvants can reduce the dose of the antigen and/or diminish the number of immunisations required for a protective vaccine response ${ }^{78}$. Considering the focus on neonatal vaccines, we were especially interested in stimulation of the subdued Th1 response in newborn pigs ${ }^{79}$. Additionally, stimulation 
of the B-cell response and activation of APCs are also desired adjuvant properties, as is discussed in the "Neonatal immune responses" section. Currently there are numerous different adjuvant formulations under research, often containing a mixture of immunostimulatory components that can be included in/ or mixed with a passive vehicle, such as an emulsion of water and oil 77,80 .

\section{Immuno-stimulator targeting Toll-Like Receptors}

The name TLR is derived from the drosophila gene Toll. TLRs are one of the best studied family of PRRs that recognize specific molecular patterns present in pathogens such as viruses, bacteria or fungi ${ }^{81,82}$. To date, 10 different TLRs have been described in pigs ${ }^{83}$, which are expressed on the cell-surface (TLR1, TLR2, TLR4, TLR5, TLR6 and TLR10) or in the intracellular compartment (TLR3, TLR7, TLR8 and TLR9). Signalling after TLR activation is different for the specific TLRs, however all of them, except for TLR3, use the MyD88-depent pathway, inducing production of inflammatory cytokines such as TNF and IL-1 $\beta$. Besides the MyD88 pathway, TLR7/8 and TLR9 also use the IRF7-pathway, inducing the production of type I interferon (IFN- $\alpha$ and IFN- $\beta)^{84}$ (Figure 3). Porcine TLR-expression can vary between different cell-types and age groups ${ }^{85}$. TLR1/2, TLR7/8 and TLR9 are expressed on a variety of immune cells, such as DCs, macrophages, T-cells and B-cells ${ }^{35,86,87}$. TLR 7/8 and TLR9 are especially highly expressed on pDCs that also express high levels of IRF-7, making pDCs the most potent IFN-a producing cell type ${ }^{88}$.

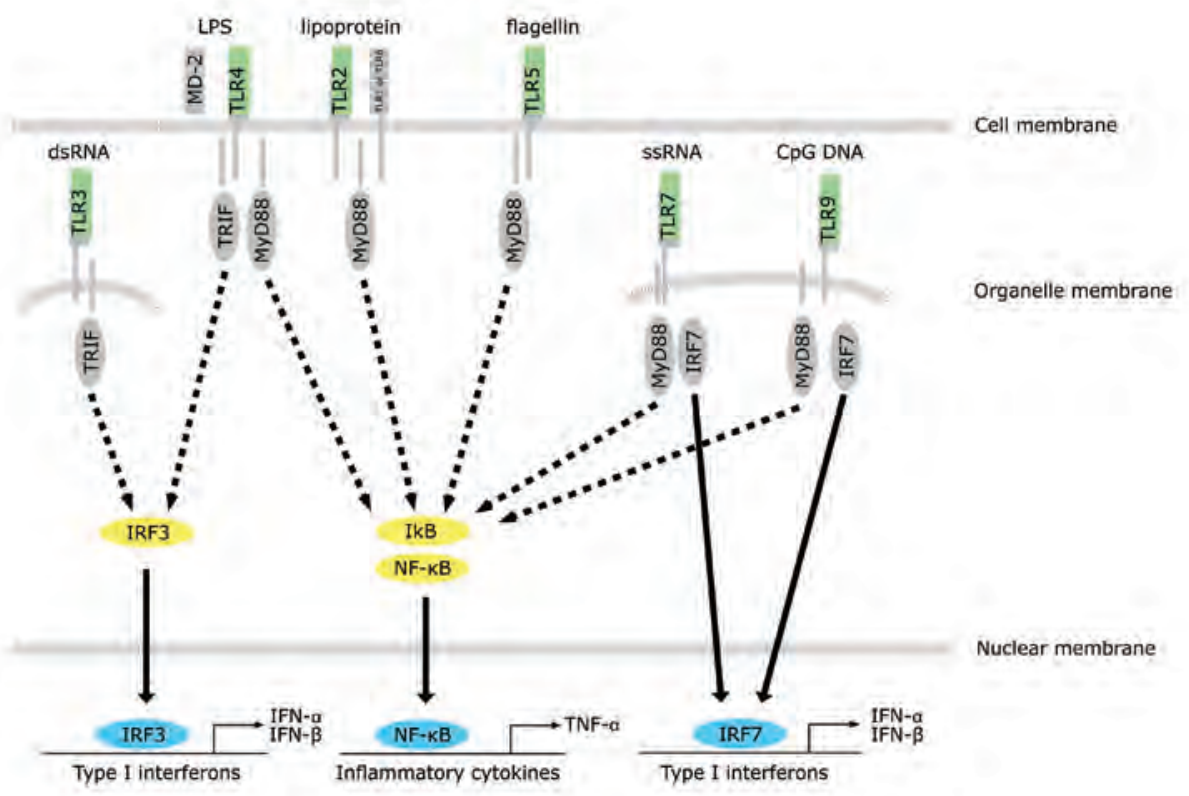

Figure 3: Overview TLR signalling.

Schematic overview of the main signalling pathways presenting the most characteristic genes of the differential TLR signalling, adapted from Uenishi ${ }^{83}$. Abbreviations: see appendix. 
Activation of the innate immune response with TLR agonists induces a cascade of responses, which will steer the adaptive immune response post-vaccination. TLR agonists have the potential to activate DCs, modulate the adaptive immune response towards different Th profiles, and promote antigen cross-presentation by $\mathrm{MHC}$ I to cytotoxic T-cells ${ }^{89,90}$. Studies have shown that porcine neonatal BDCs were stimulated by TLR7 and TLR9 agonists in a similar way as adult BDCS ${ }^{86}$, and that TLR2, TLR7/8 and TLR9 induced activation of porcine adult BDCs ${ }^{21}$. Based on the porcine studies and the promising results in other species that will be described in the next paragraph, we selected TLR2, TLR7/8 and TLR9 agonists as immuno-stimulators in this thesis.

TLR2 combines with TLR1 or TLR6 to form a cell membrane-bound heterodimer, which binds bacterial and fungal cell wall compounds. Diacyl lipoproteins are recognized by TLR2/6 (similar to the synthetic agonist Pam2Cys) and triacyl lipoproteins are recognized by TLR1/2 (similar to the synthetic Pam3Cys, named a TLR2 ligand in chapter 2 and used in this thesis) ${ }^{91}$. TLR2 agonists have the potential to stimulate the immune system as shown for veterinary vaccines ${ }^{92}$ and the human neonatal tuberculosis vaccine ${ }^{93}$. The TLR7/8 combination is not a heterodimer, but both TLR7 and TLR8 recognize single-stranded (ss) RNA and are often stimulated in combination. Several synthetic TLR7/8 agonists have been studied for their adjuvant properties in pigs and humans ${ }^{94-96}$, where they showed potency to stimulate the adaptive immune response in adults and neonates ${ }^{97,98}$. In this thesis, we tested the widely used Resiquimod (R848) for TLR7/8 stimulation. TLR9 is a receptor for unmethylated $\mathrm{CpG}$ DNA from bacteria or viruses and is mainly expressed on (p)DCs and B-cells ${ }^{84}$. Synthetic CpG oligodeoxynucleotides (ODN) stimulation can induce strong Th1 responses ${ }^{99}$ in different species ${ }^{100,101}$. Different classes $(A, B$, and $C$ ) of CpG ODN have been identified based on the CPG arrangement and immune response ${ }^{102}$. For this thesis we selected CpG ODN type-A (also named type-D), which is able to induce strong IFN-a responses ${ }^{103}$ and was successfully used in porcine studies in adults and neonates 21,86 .

\section{Emulsion based adjuvants}

Freund's complete adjuvant, the first developed emulsion adjuvant, is a water-in-mineral oil (W/O) emulsion, which induced potent immune responses, but also abscesses and granulomas due to the induction of a chronic inflammatory state at the injection site ${ }^{104}$. Oil-in-water (O/W) emulsion are more fluid and induce less reaction at the infection site, however, they are still able to support immunogenicity of vaccines ${ }^{105}$. The characteristics of an O/W emulsion is determined by the droplet-size of the mineral or non-mineral oil, the viscosity of the emulsion, and the properties of the surfactant (emulsifier). In the vaccine studies of this thesis (chapter $\mathbf{4}$ and 5) we used the commercial O/W emulsion

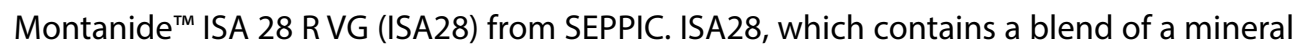
and non-mineral oil and an optimized surfactant system, and which induces a strong short-term immunity within veterinary vaccines ${ }^{106}$. Besides ISA28, O/W emulsions based 
on squalene were used as adjuvants. Squalene (originally obtained from shark liver oil) is a highly metabolizable oil that induces limited side effects, while maintaining the capacity to evoke a cellular and humoral immune response ${ }^{107}$. Two different squalene emulsions were applied: squalene-based O/W emulsion (SWE) for the intramuscular vaccination ${ }^{108}$ and a stable O/W emulsion with squalene (SE) for the skin vaccination ${ }^{109}$. Both SE and SWE were mixed with TLR1/2, 7/8 and 9 agonists to form an experimental adjuvant formulation in chapter 5.

\section{Vaccine delivery: dissolving microneedle- patches}

The skin is well supplied with APCs, consisting of LCs in the epidermis and dDCs and macrophages in the dermis. This makes the skin an attractive target for vaccine delivery because it has the advantage of giving a dose reduction of vaccine-antigen, improved immune responses and needle-free delivery (less painful and can be applied by a less qualified person) compared to traditional intramuscular or subcutaneous delivery ${ }^{110,111}$. The terms used to describe skin vaccination are not standard and depend on which part of the skin (epidermis, dermis, or both) are targeted. Examples of terminology reflecting the route of vaccine delivery are: transcutaneous (surface of skin), epidermal (epidermis), intradermal (dermis), percutaneous (epidermis and dermis), cutaneous (applicable for epidermal, intradermal and transcutaneous) and transdermal (transcutaneous or epidermal) ${ }^{112,113}$. In this thesis we used dissolving microneedle (DMN) patches ${ }^{114}$, which are applied on the surface of the skin where the small needles (500-600 $\mu \mathrm{m}$ in length) penetrate the epidermis and superficial dermis. Because of this, a percutaneous or cutaneous vaccination type might be appropriate terms to refer to method of delivery. However, to avoid confusion we use "skin vaccination" to indicate vaccine delivery in the skin with DMN-patches in this thesis.

\section{Outline of thesis}

The objective of this thesis was to investigate and to improve the understanding of vaccineinduced immune responses in neonatal pigs by comparing their immune responses with adult pigs. Three different TLR agonists were applied as immuno-stimulators, and skin vaccination was used as an additional delivery route to intramuscular vaccination. By using the iPRRSV-antigen with weak immunogenic properties, the TLR receptor stimulation would be an essential prerequisite to provoke an effective immune response in the different age groups. Skin vaccination was used to study the immunogenic potential of this delivery route, and to increase our knowledge about porcine neonatal skin immunity for which only limited information is currently available. In chapter 1 I discussed the aim and outline of this thesis, and introduced relevant background information for the different studies: the DCs, vaccine immunology in neonatal and adult pigs and vaccine formulations/delivery. Table 3 provides an overview of the different immune responses studied in this thesis after TLR stimulation and/or vaccination. 
Table 3: Immune responses studied in this thesis.

\begin{tabular}{|c|c|c|c|}
\hline & Immune response & Type of immune cells or sample & Assay \\
\hline \multirow[t]{3}{*}{ Chapter 2} & DC activation & DCs and monocytes & $\begin{array}{l}\text { Flow cytometry: DC subsets (Table } \\
\text { 1) and intracellular TNF staining }\end{array}$ \\
\hline & $\begin{array}{l}\text { Th1 and Th2 } \\
\text { directed cytokines }\end{array}$ & PBMC & $\begin{array}{l}\text { Cytokine production } \\
\text { (Luminex) }\end{array}$ \\
\hline & & $\begin{array}{l}\text { mRNA from enriched } \\
\text { mononuclear cells (PBMC/CD3-) }\end{array}$ & $\begin{array}{l}\text { mRNA upregulation cytokines } \\
\text { (qPCR) and TLR expression }\end{array}$ \\
\hline \multirow[t]{3}{*}{ Chapter 3} & $\begin{array}{l}\text { Innate immune } \\
\text { response skin }\end{array}$ & $\begin{array}{l}\text { Neutrophils and } \\
\text { mononuclear cells (in situ) }\end{array}$ & histopathology \\
\hline & APC activation & DCs and M $\phi$ in skin in situ & IF microscopy and IHC \\
\hline & $\begin{array}{l}\text { general innate } \\
\text { immune response }\end{array}$ & $\begin{array}{l}\text { mRNA from vaccinated skin and } \\
\text { draining lymph node }\end{array}$ & $\begin{array}{l}\text { Transcriptome analysis } \\
\text { Fluidigm }\end{array}$ \\
\hline \multirow[t]{3}{*}{ Chapter 4/5 } & $\begin{array}{l}\text { Specific Humoral } \\
\text { immune response } \\
\text { (Th2 response) }\end{array}$ & $\begin{array}{l}\text { Serum, PRRSV specific lgG } \\
\text { production by plasma cells }\end{array}$ & ELISA \\
\hline & $\begin{array}{l}\text { Th1 memory } \\
\text { response (cellular } \\
\text { immune response) }\end{array}$ & Th1 memory cells and NK cells & IFN-Y ELIspot \\
\hline & $\begin{array}{l}\text { Protective immune } \\
\text { response }\end{array}$ & PRRSV titer in serum & Virus titration \\
\hline \multirow[t]{2}{*}{ Chapter 5} & $\begin{array}{l}\text { Th1/2/17 directed } \\
\text { innate immune } \\
\text { response }\end{array}$ & PBMC & Cytokine production (Luminex): \\
\hline & $\begin{array}{l}\text { Th1 memory } \\
\text { response }\end{array}$ & $\begin{array}{l}\text { Naive } \mathrm{CD}^{+} \text {-cells, Th memory } \\
\text { cells and cytotoxic T-cells } \\
\text { and B-cells }\end{array}$ & $\begin{array}{l}\text { Flow cytometry: T-cell subsets with } \\
\text { intracellular staining for TNF and } \\
\text { IFN-Y and B-cells }\end{array}$ \\
\hline
\end{tabular}

Abbreviations: see appendix.

We started this project with in vitro research, where we investigated the potential of the TLR1/2 and TLR9 agonists that were rationally selected, to stimulate blood of adult and neonatal pigs (chapter 2). In that chapter we focused on the activation of DCs, which are an important link between the innate and adaptive immune response and thereby essential for the induction of adequate vaccine responses. Based on these results, three different TLR agonists were selected as possible effective immuno-stimulators (adjuvants) in an iPRRSV vaccine for adult and neonatal pigs. In chapter $\mathbf{3}$ we investigated the early and local immune response after skin vaccination with different DMN-patches in neonatal and adult pigs. There, the selected TLR agonists were combined with iPRRSV-antigen in DMN-patches and adult and neonatal pigs were immunized in the skin with three different vaccine preparations containing TLR1/2, 7/8 or 9 agonists as adjuvants. Local immune responses after DMN-patch application were assessed in the skin and draining lymph node $24 \mathrm{~h}$ after vaccination. Additionally, non-vaccinated or normal skin and lymph nodes of adult and neonatal pigs were compared to identify possible age-depend differences, which could influence the effect of skin vaccination. 
Besides the local innate immune response, it is essential that a vaccine establishes a systemic and specific immune response and specifically protects against the potential pathogen. In a prime-booster vaccination experiment followed by a challenge with PRRSV in adult pigs (chapter 4) we assessed the immunogenicity and efficacy of the three different DMN-patches used in chapter 3. In order to determine the potency of skin vaccination, the same vaccine formulations as incorporated in the DMN-patches were applied via the intramuscular route in adult pigs. The vaccine-antigen and the prime, booster and challenge regimen for the neonatal follow-up study (chapter 5) were identical to the adult study in chapter 4, only different adjuvant formulations were used for the neonatal study in order to increase the efficacy of the iPRRSV vaccine. Neonatal pigs were vaccinated with an adjuvant formulation composed of a mixture of the three previously used TLR agonists combined with a squalene-in-water emulsion (SWE) and iPRRSV. This vaccine was delivered in the skin with DMN-patches and also intramuscularly. Corresponding assays as those employed in the adult vaccination experiment were used to assess the immunogenicity and efficacy of the vaccines after a prime vaccination on 3-days of age. Chapter $\mathbf{6}$ summarizes and discusses the main findings of this thesis with suggestions for future research. 


\section{References}

1 Nieuwenhuis, N., Duinhof, T. F. \& van Nes, A. Economic analysis of outbreaks of porcine reproductive and respiratory syndrome virus in nine sow herds. The Veterinary record 170, 225, doi:10.1136/vr.100101 (2012).

2 Nathues, H. et al. Modelling the economic efficiency of using different strategies to control Porcine Reproductive \& Respiratory Syndrome at herd level. Preventive veterinary medicine 152, 89-102, doi:10.1016/j.prevetmed.2018.02.005 (2018).

3 Schulz, L. L. \& Tonsor, G. T. Assessment of the economic impacts of porcine epidemic diarrhea virus in the United States. J. Anim. Sci. 93, 5111-5118, doi:10.2527/jas.2015-9136 (2015).

4 Alarcon, P., Rushton, J. \& Wieland, B. Cost of post-weaning multi-systemic wasting syndrome and porcine circovirus type-2 subclinical infection in England - an economic disease model. Preventive veterinary medicine 110, 88-102, doi:10.1016/j.prevetmed.2013.02.010 (2013).

5 Rose, N. \& Andraud, M. The use of vaccines to control pathogen spread in pig populations. Porcine health management 3, 8, doi:10.1186/s40813-017-0053-6 (2017).

6 Murphy, K. \& Weaver, C. Janeway's immunobiology. (2017).

7 Banchereau, J. \& Steinman, R. M. Dendritic cells and the control of immunity. Nature 392, 245-252, doi:10.1038/32588 (1998).

8 Uenishi, H., Shinkai, H., Morozumi, T. \& Muneta, Y. Genomic survey of polymorphisms in pattern recognition receptors and their possible relationship to infections in pigs. Veterinary immunology and immunopathology 148, 69-73, doi:10.1016/j.vetimm.2011.07.019 (2012).

9 Randolph, G. J., Ochando, J. \& Partida-Sanchez, S. Migration of dendritic cell subsets and their precursors. Annual review of immunology 26, 293-316, doi:10.1146/annurev.immunol.26.021607.090254 (2008).

10 Forster, R., Braun, A. \& Worbs, T. Lymph node homing of T cells and dendritic cells via afferent lymphatics. Trends in immunology 33, 271-280, doi:10.1016/j.it.2012.02.007 (2012).

11 Banchereau, J. et al. Immunobiology of Dendritic Cells. Annu. Rev. Immunol. 18, 767-811, doi:10.1146/ annurev.immunol.18.1.767 (2000).

12 Collin, M. \& Bigley, V. Human dendritic cell subsets: an update. Immunology 154, 3-20, doi:10.1111/ imm.12888 (2018).

13 Segura, E. in Dendritic Cell Protocols (eds Elodie Segura \& Nobuyuki Onai) 3-15 (Springer New York, 2016).

14 Summerfield, A. \& McCullough, K. C. The porcine dendritic cell family. Developmental and comparative immunology 33, 299-309, doi:10.1016/j.dci.2008.05.005 (2009).

15 Franzoni, G., Graham, S. P., Dei Giudici, S. \& Oggiano, A. Porcine Dendritic Cells and Viruses: An Update. Viruses 11, doi:10.3390/v11050445 (2019).

16 Carrasco, C. P. et al. Porcine dendritic cells generated in vitro: morphological, phenotypic and functional properties. Immunology 104, 175-184, doi:10.1046/j.1365-2567.2001.01299.x (2001).

17 Paillot, R., Laval, F., Audonnet, J.-C., Andreoni, C. \& Juillard, V. Functional and phenotypic characterization of distinct porcine dendritic cells derived from peripheral blood monocytes. Immunology 102, 396-404, doi:10.1046/j.1365-2567.2001.01200.x (2001).

18 Schlitzer, A., McGovern, N. \& Ginhoux, F. Dendritic cells and monocyte-derived cells: Two complementary and integrated functional systems. Semin. Cell Dev. Biol. 41, 9-22, doi:10.1016/j.semcdb.2015.03.011 (2015). 
19 Guzylack-Piriou, L., Alves, M. P., McCullough, K. C. \& Summerfield, A. Porcine Flt3 ligand and its receptor: generation of dendritic cells and identification of a new marker for porcine dendritic cells. Developmental and comparative immunology 34, 455-464, doi:10.1016/j.dci.2009.12.006 (2010).

20 Summerfield, A. et al. Porcine peripheral blood dendritic cells and natural interferon-producing cells. Immunology 110, 440-449 (2003).

21 Auray, G. et al. Characterization and Transcriptomic Analysis of Porcine Blood Conventional and Plasmacytoid Dendritic Cells Reveals Striking Species-Specific Differences. Journal of immunology 197, 4791-4806, doi:10.4049/jimmunol.1600672 (2016).

22 Summerfield, A., Auray, G. \& Ricklin, M. Comparative dendritic cell biology of veterinary mammals. Annual review of animal biosciences 3, 533-557, doi:10.1146/annurev-animal-022114-111009 (2015).

23 Parra-Sanchez, H., Puebla-Clark, L., Resendiz, M., Valenzuela, O. \& Hernandez, J. Characterization and expression of DEC205 in the CDC1 and CDC2 subsets of porcine dendritic cells from spleen, tonsil, and submaxillary and mesenteric lymph nodes. Molecular immunology 96, 1-7, doi:10.1016/j. molimm.2018.02.003 (2018).

24 Dutertre, C. A., Wang, L. F. \& Ginhoux, F. Aligning bona fide dendritic cell populations across species. Cell. Immunol. 291, 3-10, doi:10.1016/j.cellimm.2014.08.006 (2014).

25 Murata, Y., Kotani, T., Ohnishi, H. \& Matozaki, T. The CD47-SIRPalpha signalling system: its physiological roles and therapeutic application. J Biochem 155, 335-344, doi:10.1093/jb/mvu017 (2014).

26 Khiao In, M. et al. Histological and functional comparisons of four anatomical regions of porcine skin with human abdominal skin. Anatomia, histologia, embryologia 48, 207-217, doi:10.1111/ahe.12425 (2019).

27 Haniffa, M., Gunawan, M. \& Jardine, L. Human skin dendritic cells in health and disease. J Dermatol Sci 77, 85-92, doi:10.1016/j.jdermsci.2014.08.012 (2015).

28 Collin, M. \& Milne, P. Langerhans cell origin and regulation. Curr. Opin. Hematol. 23, 28-35, doi:10.1097/ moh.0000000000000202 (2016).

29 Summerfield, A., Meurens, F. \& Ricklin, M. E. The immunology of the porcine skin and its value as a model for human skin. Molecular immunology 66, 14-21, doi:10.1016/j.molimm.2014.10.023 (2015).

30 Kashem, S. W., Haniffa, M. \& Kaplan, D. H. Antigen-Presenting Cells in the Skin. Annual review of immunology 35, 469-499, doi:10.1146/annurev-immunol-051116-052215 (2017).

31 Fehres, C. M. et al. Phenotypic and Functional Properties of Human Steady State CD14+ and CD1a+ Antigen Presenting Cells and Epidermal Langerhans Cells. PloS one 10, e0143519, doi:10.1371/journal. pone.0143519 (2015).

32 Marquet, F. et al. Pig skin includes dendritic cell subsets transcriptomically related to human CD1a and CD14 dendritic cells presenting different migrating behaviors and T cell activation capacities. Journal of immunology 193, 5883-5893, doi:10.4049/jimmunol.1303150 (2014).

33 Marquet, F. et al. Characterization of dendritic cells subpopulations in skin and afferent lymph in the swine model. PloS one 6, e16320, doi:10.1371/journal.pone.0016320 (2011).

Mair, K. H. et al. The porcine innate immune system: an update. Developmental and comparative immunology 45, 321-343, doi:10.1016/j.dci.2014.03.022 (2014).

35 Iwasaki, A. \& Medzhitov, R. Control of adaptive immunity by the innate immune system. Nature immunology 16, 343-353, doi:10.1038/ni.3123 (2015). 
36 Ebner, F. et al. Pathogen-Reactive T Helper Cell Analysis in the Pig. Frontiers in immunology 8, 565, doi:10.3389/fimmu.2017.00565 (2017).

37 Romagnani, S. T-cell subsets (Th1 versus Th2). Ann Allergy Asthma Immunol 85, 9-18; quiz 18, 21, doi:10.1016/s1081-1206(10)62426-x (2000).

38 Pulendran, B. \& Ahmed, R. Immunological mechanisms of vaccination. Nature immunology 12, 509-517, doi:10.1038/ni.2039 (2011).

39 Mohr, E. \& Siegrist, C. A. Vaccination in early life: standing up to the challenges. Current opinion in immunology 41, 1-8, doi:10.1016/j.coi.2016.04.004 (2016).

40 Saso, A. \& Kampmann, B. Vaccine responses in newborns. Seminars in immunopathology 39, 627-642, doi:10.1007/s00281-017-0654-9 (2017).

41 Meurens, F., Summerfield, A., Nauwynck, H., Saif, L. \& Gerdts, V. The pig: a model for human infectious diseases. Trends Microbiol. 20, 50-57, doi:10.1016/j.tim.2011.11.002 (2012).

42 Levast, B., Schulz, S., Hurk, S. \& Gerdts, V. Animal models for neonatal diseases in humans. Vaccine 31, 24892499, doi:10.1016/j.vaccine.2012.11.089 (2013).

43 Kumar, S. K. \& Bhat, B. V. Distinct mechanisms of the newborn innate immunity. Immunol Lett 173, 42-54, doi:10.1016/j.imlet.2016.03.009 (2016).

44 Kollmann, T. R. et al. Neonatal innate TLR-mediated responses are distinct from those of adults. Journal of immunology 183, 7150-7160, doi:10.4049/jimmunol.0901481 (2009).

45 Zaghouani, H., Hoeman, C. M. \& Adkins, B. Neonatal immunity: faulty T-helpers and the shortcomings of dendritic cells. Trends in immunology 30, 585-591, doi:10.1016/j.it.2009.09.002 (2009).

46 Levy, O. Innate immunity of the newborn: basic mechanisms and clinical correlates. Nature reviews. Immunology 7, 379-390, doi:10.1038/nri2075 (2007).

47 Debock, I. \& Flamand, V. Unbalanced Neonatal CD4+ T-Cell Immunity. Front. Immunol. 5, 393, doi:10.3389/ fimmu.2014.00393 (2014).

48 Lindenstrom, T. et al. Vaccine-induced th17 cells are maintained long-term postvaccination as a distinct and phenotypically stable memory subset. Infect. Immun. 80, 3533-3544, doi:10.1128/iai.00550-12 (2012).

49 McGeachy, M. J. Th17 memory cells: live long and proliferate. Journal of leukocyte biology 94, 921-926, doi:10.1189/jlb.0313113 (2013).

50 Sinkora, M. \& Butler, J. E. The ontogeny of the porcine immune system. Developmental and comparative immunology 33, 273-283, doi:10.1016/j.dci.2008.07.011 (2009).

51 Siegrist, C. A. \& Aspinall, R. B-cell responses to vaccination at the extremes of age. Nature reviews. Immunology 9, 185-194, doi:10.1038/nri2508 (2009).

52 Vono, M. et al. Overcoming the Neonatal Limitations of Inducing Germinal Centers through LiposomeBased Adjuvants Including C-Type Lectin Agonists Trehalose Dibehenate or Curdlan. Frontiers in immunology 9, 381, doi:10.3389/fimmu.2018.00381 (2018).

53 Butler, J. E. et al. The piglet as a model for B cell and immune system development. Veterinary immunology and immunopathology 128, 147-170, doi:10.1016/j.vetimm.2008.10.321 (2009).

54 Sinkora, J. et al. Expression of CD2 on porcine B lymphocytes. Immunology 95, 443-449, doi:10.1046/j.13652567.1998.00621.x (1998). 
55 Sinkora, J., Rehakova, Z., Sinkora, M., Cukrowska, B. \& Tlaskalova-Hogenova, H. Early development of immune system in pigs. Veterinary immunology and immunopathology 87, 301-306, doi:10.1016/s01652427(02)00056-9 (2002).

56 Willems, F., Vollstedt, S. \& Suter, M. Phenotype and function of neonatal DC. European journal of immunology 39, 26-35, doi:10.1002/eji.200838391 (2009).

57 Velilla, P. A., Rugeles, M. T. \& Chougnet, C. A. Defective antigen-presenting cell function in human neonates. Clinical immunology (Orlando, Fla.) 121, 251-259, doi:10.1016/j.clim.2006.08.010 (2006).

58 Borras, F. E., Matthews, N. C., Lowdell, M. W. \& Navarrete, C. V. Identification of both myeloid CD11C+ and lymphoid CD11c- dendritic cell subsets in cord blood. Br. J. Haematol. 113, 925-931, doi:10.1046/j.13652141.2001.02840.x (2001).

59 Debeer, S. et al. Comparative histology and immunohistochemistry of porcine versus human skin. Eur. J. Dermatol. 23, 456-466, doi:10.1684/ejd.2013.2060 (2013).

60 Visscher, M. \& Narendran, V. The Ontogeny of Skin. Advances in wound care 3, 291-303, doi:10.1089/ wound.2013.0467 (2014).

61 Elbe-Burger, A. \& Schuster, C. Development of the prenatal cutaneous antigen-presenting cell network. Immunology and cell biology 88, 393-399, doi:10.1038/icb.2010.13 (2010).

62 Narendran, V., Visscher, M. O., Abril, I., Hendrix, S. W. \& Hoath, S. B. Biomarkers of epidermal innate immunity in premature and full-term infants. Pediatric research 67, 382-386, doi:10.1203/PDR.0b013e3181d00b73 (2010).

63 Rossow, K. D. Porcine reproductive and respiratory syndrome. Veterinary pathology 35, 1-20, doi:10.1177/030098589803500101 (1998).

64 King, A. M. Q. et al. Changes to taxonomy and the International Code of Virus Classification and Nomenclature ratified by the International Committee on Taxonomy of Viruses (2018). Archives of virology, doi:10.1007/s00705-018-3847-1 (2018).

65 Loving, C. L., Osorio, F. A., Murtaugh, M. P. \& Zuckermann, F. A. Innate and adaptive immunity against Porcine Reproductive and Respiratory Syndrome Virus. Veterinary immunology and immunopathology 167, 1-14, doi:10.1016/j.vetimm.2015.07.003 (2015).

66 Butler, J. E. et al. Porcine reproductive and respiratory syndrome (PRRS): an immune dysregulatory pandemic. Immunol. Res. 59, 81-108, doi:10.1007/s12026-014-8549-5 (2014).

67 Lunney, J. K. et al. Porcine Reproductive and Respiratory Syndrome Virus (PRRSV): Pathogenesis and Interaction with the Immune System. Annual review of animal biosciences 4, 129-154, doi:10.1146/annurevanimal-022114-111025 (2016).

68 Rahe, M. C. \& Murtaugh, M. P. Effector mechanisms of humoral immunity to porcine reproductive and respiratory syndrome virus. Veterinary immunology and immunopathology 186, 15-18, doi:10.1016/j. vetimm.2017.02.002 (2017).

69 Burkard, C. et al. Precision engineering for PRRSV resistance in pigs: Macrophages from genome edited pigs lacking CD163 SRCR5 domain are fully resistant to both PRRSV genotypes while maintaining biological function. PLoS Pathog. 13, e1006206, doi:10.1371/journal.ppat.1006206 (2017).

70 Crisci, E., Fraile, L. \& Montoya, M. Cellular Innate Immunity against PRRSV and Swine Influenza Viruses. Veterinary sciences 6, doi:10.3390/vetsci6010026 (2019). 
71 Kappes, M. A. \& Faaberg, K. S. PRRSV structure, replication and recombination: Origin of phenotype and genotype diversity. Virology 479-480, 475-486, doi:10.1016/j.virol.2015.02.012 (2015).

72 European Medicines Agency, E. CVMP recommendation on the use of live attenuated PRRSV vaccines Committee for Medicinal Products for Veterinary Use (CVMP) meeting of 3-5 December 2019 (2019).

73 Nan, Y. et al. Improved Vaccine against PRRSV: Current Progress and Future Perspective. Frontiers in microbiology 8, 1635, doi:10.3389/fmicb.2017.01635 (2017).

74 Vanhee, M., Delputte, P. L., Delrue, I., Geldhof, M. F. \& Nauwynck, H. J. Development of an experimental inactivated PRRSV vaccine that induces virus-neutralizing antibodies. Veterinary research 40, 63, doi:10.1051/vetres/2009046 (2009).

75 Geldhof, M. F. et al. Comparison of the efficacy of autogenous inactivated Porcine Reproductive and Respiratory Syndrome Virus (PRRSV) vaccines with that of commercial vaccines against homologous and heterologous challenges. BMC veterinary research 8, 182, doi:10.1186/1746-6148-8-182 (2012).

76 Charerntantanakul, W. Adjuvants for porcine reproductive and respiratory syndrome virus vaccines. Veterinary immunology and immunopathology 129, 1-13, doi:10.1016/j.vetimm.2008.12.018 (2009).

77 Savelkoul, H. F., Ferro, V. A., Strioga, M. M. \& Schijns, V. E. Choice and Design of Adjuvants for Parenteral and Mucosal Vaccines. Vaccines 3, 148-171, doi:10.3390/vaccines3010148 (2015).

78 Kasturi, S. P. et al. Programming the magnitude and persistence of antibody responses with innate immunity. Nature 470, 543-547, doi:10.1038/nature09737 (2011).

79 Duthie, M. S., Windish, H. P., Fox, C. B. \& Reed, S. G. Use of defined TLR ligands as adjuvants within human vaccines. Immunol. Rev. 239, 178-196, doi:10.1111/j.1600-065X.2010.00978.x (2011).

80 McKee, A. S. \& Marrack, P. Old and new adjuvants. Current opinion in immunology 47, 44-51, doi:10.1016/j. coi.2017.06.005 (2017).

81 Akira, S. \& Takeda, K. Toll-like receptor signalling. Nature reviews. Immunology 4, 499-511, doi:10.1038/ nri1391 (2004).

82 Anthoney, N., Foldi, I. \& Hidalgo, A. Toll and Toll-like receptor signalling in development. Development 145, doi:10.1242/dev.156018 (2018).

83 Uenishi, H. \& Shinkai, H. Porcine Toll-like receptors: the front line of pathogen monitoring and possible implications for disease resistance. Developmental and comparative immunology 33, 353-361, doi:10.1016/j. dci.2008.06.001 (2009).

84 Takeda, K. \& Akira, S. Toll-like receptors in innate immunity. International immunology 17, 1-14, doi:10.1093/ intimm/dxh186 (2005).

85 Uddin, M. J. et al. Expression patterns of porcine Toll-like receptors family set of genes (TLR1-10) in gut-associated lymphoid tissues alter with age. Research in veterinary science 95, 92-102, doi:10.1016/j. rvsc.2013.01.027 (2013).

86 Auray, G. et al. Porcine neonatal blood dendritic cells, but not monocytes, are more responsive to TLRs stimulation than their adult counterparts. PloS one 8, e59629, doi:10.1371/journal.pone.0059629 (2013).

87 Braun, R. O., Python, S. \& Summerfield, A. Porcine B Cell Subset Responses to Toll-like Receptor Ligands. Frontiers in immunology 8, 1044, doi:10.3389/fimmu.2017.01044 (2017).

88 Fitzgerald-Bocarsly, P., Dai, J. \& Singh, S. Plasmacytoid dendritic cells and type I IFN: 50 years of convergent history. Cytokine \& growth factor reviews 19, 3-19, doi:10.1016/j.cytogfr.2007.10.006 (2008). 
89 Maisonneuve, C., Bertholet, S., Philpott, D. J. \& De Gregorio, E. Unleashing the potential of NOD- and Tolllike agonists as vaccine adjuvants. Proceedings of the National Academy of Sciences of the United States of America 111, 12294-12299, doi:10.1073/pnas.1400478111 (2014).

90 Lahiri, A., Das, P. \& Chakravortty, D. Engagement of TLR signaling as adjuvant: towards smarter vaccine and beyond. Vaccine 26, 6777-6783, doi:10.1016/j.vaccine.2008.09.045 (2008).

91 Oliveira-Nascimento, L., Massari, P. \& Wetzler, L. M. The Role of TLR2 in Infection and Immunity. Frontiers in immunology 3, 79, doi:10.3389/fimmu.2012.00079 (2012).

92 Basto, A. P. \& Leitao, A. Targeting TLR2 for vaccine development. Journal of immunology research 2014, 619410, doi:10.1155/2014/619410 (2014).

93 Kativhu, C. L. \& Libraty, D. H. A Model to Explain How the Bacille Calmette Guerin (BCG) Vaccine Drives Interleukin-12 Production in Neonates. PloS one 11, e0162148, doi:10.1371/journal.pone.0162148 (2016).

94 McKay, P. F. et al. TLR4 and TLR7/8 Adjuvant Combinations Generate Different Vaccine Antigen-Specific Immune Outcomes in Minipigs when Administered via the ID or IN Routes. PloS one 11, e0148984, doi:10.1371/journal.pone.0148984 (2016).

95 Smith, A. J. et al. Evaluation of novel synthetic TLR7/8 agonists as vaccine adjuvants. Vaccine 34, 4304-4312, doi:10.1016/j.vaccine.2016.06.080 (2016).

96 Dowling, D. J. Recent Advances in the Discovery and Delivery of TLR7/8 Agonists as Vaccine Adjuvants. Immunohorizons 2, 185-197, doi:10.4049/immunohorizons.1700063 (2018).

97 Dowling, D. J.et al. TLR7/8 adjuvant overcomes newborn hyporesponsiveness to pneumococcal conjugate vaccine at birth. JCl insight 2, e91020, doi:10.1172/jci.insight.91020 (2017).

van Haren, S. D. et al. Age-Specific Adjuvant Synergy: Dual TLR7/8 and Mincle Activation of Human Newborn Dendritic Cells Enables Th1 Polarization. Journal of immunology 197, 4413-4424, doi:10.4049/ jimmunol.1600282 (2016).

99 Heeg, K. \& Zimmermann, S. CpG DNA as a Th1 trigger. International archives of allergy and immunology 121, 87-97, doi:10.1159/000024303 (2000).

100 Shi, S. et al. Vaccine adjuvants: Understanding the structure and mechanism of adjuvanticity. Vaccine 37, 3167-3178, doi:10.1016/j.vaccine.2019.04.055 (2019).

101 Van der Stede, Y., Verdonck, F., Vancaeneghem, S., Cox, E. \& Goddeeris, B. M. CpG-oligodinucleotides as an effective adjuvant in pigs for intramuscular immunizations. Veterinary immunology and immunopathology 86, 31-41 (2002).

102 Scheiermann, J. \& Klinman, D. M. Clinical evaluation of CpG oligonucleotides as adjuvants for vaccines targeting infectious diseases and cancer. Vaccine 32, 6377-6389, doi:10.1016/j.vaccine.2014.06.065 (2014).

103 Hartmann, E. et al. Analysis of plasmacytoid and myeloid dendritic cells in nasal epithelium. Clinical and vaccine immunology: CVI 13, 1278-1286, doi:10.1128/CVI.00172-06 (2006).

104 Martinon, S. et al. Chemical and Immunological Characteristics of Aluminum-Based, Oil-Water Emulsion, and Bacterial-Origin Adjuvants. Journal of immunology research 2019, 3974127, doi:10.1155/2019/3974127 (2019).

105 Aucouturier, J., Dupuis, L. \& Ganne, V. Adjuvants designed for veterinary and human vaccines. Vaccine 19, 2666-2672 (2001). 
106 Ibrahim Eel, S. et al. Comparative study on the immunopotentiator effect of ISA 201, ISA 61, ISA 50, ISA 206 used in trivalent foot and mouth disease vaccine. Vet World 8, 1189-1198, doi:10.14202/ vetworld.2015.1189-1198 (2015).

107 Fox, C. B. \& Haensler, J. An update on safety and immunogenicity of vaccines containing emulsion-based adjuvants. Expert review of vaccines 12, 747-758, doi:10.1586/14760584.2013.811188 (2013).

108 Ventura, R. et al. Technology transfer of an oil-in-water vaccine-adjuvant for strengthening pandemic influenza preparedness in Indonesia. Vaccine 31, 1641-1645, doi:10.1016/j.vaccine.2012.07.074 (2013).

109 Shah, R. R. et al. The development of self-emulsifying oil-in-water emulsion adjuvant and an evaluation of the impact of droplet size on performance. Journal of pharmaceutical sciences 104, 1352-1361, doi:10.1002/ jps.24337 (2015).

110 Prausnitz, M. R. Engineering Microneedle Patches for Vaccination and Drug Delivery to Skin. Annu Rev Chem Biomol Eng 8, 177-200, doi:10.1146/annurev-chembioeng-060816-101514 (2017).

$111 \mathrm{Haq}, \mathrm{M}$. I. et al. Clinical administration of microneedles: skin puncture, pain and sensation. Biomedical microdevices 11, 35-47, doi:10.1007/s10544-008-9208-1 (2009).

112 Nicolas, J. F. \& Guy, B. Intradermal, epidermal and transcutaneous vaccination: from immunology to clinical practice. Expert review of vaccines 7, 1201-1214, doi:10.1586/14760584.7.8.1201 (2008).

113 Lambert, P. H. \& Laurent, P. E. Intradermal vaccine delivery: will new delivery systems transform vaccine administration? Vaccine 26, 3197-3208, doi:10.1016/j.vaccine.2008.03.095 (2008).

114 Marshall, S., Sahm, L. J. \& Moore, A. C. The success of microneedle-mediated vaccine delivery into skin. Hum. Vaccin. Immunother., 0, doi:10.1080/21645515.2016.1171440 (2016). 


\section{Neonatal porcine blood derived dendritic cell subsets show activation after TLR2 or TLR9 stimulation}

Sandra Vreman', Gael Auray 3,4 , Huub F.J. Savelkoul ${ }^{3}$, Annemarie Rebel', Artur Summerfield ${ }^{3,4}$, Norbert Stockhofe-Zurwieden ${ }^{1}$

1 Wageningen Bioveterinary Research, Wageningen University \& Research, P.O. Box 29703, 2502 LS The Hague, The Netherlands

${ }^{2}$ Cell Biology \& Immunology group, Wageningen University \& Research P.O. Box 338, 6700 HA Wageningen, The Netherlands

${ }^{3}$ Institute of Virology and Immunology, Sensemattstrasse 293, 3147 Mittelhausern, Switzerland

${ }^{4}$ Department of Infectious Diseases and Pathobiology, University of Bern, Switzerland 


\section{Abstract}

The present study investigated the innate immune response in vitro to determine porcine neonate responses with Toll-like receptor (TLR)2 ligand (Pam3Cys) or TLR9 ligand (CpG) and compared these with adults. We identified the same phenotypically defined dendritic cell (DC) subsets and DC proportions in porcine neonate and adult blood by flow cytometry, which were plasmacytoid DCs ( $\mathrm{pDCs}$ : $\mathrm{CD} 144^{-} \mathrm{CD} 4^{+} \mathrm{CD} 172 \mathrm{a}^{+} \mathrm{CADM1}$ ) and conventional DCs (CDCs), being further divided into a $\mathrm{CDC} 1\left(\mathrm{CD} 14 \mathrm{CD} 4 \mathrm{CD} 172 \mathrm{a}^{\text {low }} \mathrm{CADM} 1^{+}\right)$and a $\mathrm{CDC} 2$ $\left(C D 14-C D 4-C D 172 a^{+} C A D M 1^{+}\right)$subset. With neonatal cells, the TLR2 ligand induced a stronger TNF expression in monocytes and pDCs, and a stronger CD80/86 upregulation in CDC1, when compared to adult cells. Furthermore, in neonatal mononuclear cells TLR9 ligand was more potent at inducing IL12p40 mRNA expression. These results indicate clear responses of porcine neonatal antigen presenting cells after TLR2 and TLR9 stimulation, suggesting that corresponding ligands could be promising candidates for neonatal adjuvant application. 


\section{Introduction}

The early-life is a critical period characterized by high exposure to pathogens and subsequent development of infectious diseases. In swine farms diseased newborns and young piglets show a high mortality rate, causing severe welfare problems and economic losses. Vaccination is one of the most powerful strategies to protect against early-life infections ${ }^{1,2}$. However, due to differences in both innate and adaptive immune response in neonates and adults ${ }^{3,4}$ and the presence of maternal derived antibodies ${ }^{5}$ vaccines are less effective in neonates.

Newborns have experienced minimal antigen exposure in utero and depend heavily on their innate immune system. The neonatal innate response is characterized by cytokine patterns that are different from the response in adults ${ }^{6,7}$, often resulting in a less effective Th1 response ${ }^{8,9}$. Stimulation of the neonate innate immune system can provide insights for application of novel adjuvants, such as synthetic Toll like receptor (TLR) ligands 10,11, and can be a promising strategy to strengthen the immune response to vaccination of different age groups and induce a more effective Th1 or Th1/Th2 balanced response.

In vitro studies with human blood cells have already demonstrated that neonates respond differently after specific TLR stimulation ${ }^{8}$. In general, neonatal cord blood cells produce less IFN- $a$ after stimulation ${ }^{12,13}$; while IL-6 response of peripheral blood mononuclear cells (PBMCs) appears stronger in neonates compared to adults, ${ }^{14}$. Nonetheless, adult-like responses after TLR stimulation in human cord blood and in goat kids PBMCs can also be reached when cells are appropriately stimulated ${ }^{15-17}$. Therefore, TLR ligands are interesting candidates for adjuvant application in neonatal vaccination.

The main target of vaccine adjuvants are dendritic cells (DCs), potent antigen presenting cells (APCs), which are an essential link between the innate and adaptive immune response ${ }^{18,19}$. After stimulation, DCs mature and migrate to the draining lymph node to activate Th cells and induce an adaptive immune response. Different DC subsets have recently been described in porcine adult PBMCs: plasmacytoid DCs (pDCs: CD14 $\left.\mathrm{CD}^{+}{ }^{+} \mathrm{CD} 172 \mathrm{a}^{+} \mathrm{CADM1}^{-}\right)$and conventional DCs (CDCs), being further divided into a $\mathrm{CDC} 1$ $\left(C D 14-C D 4-C D 172 a^{\text {low }}\right.$ CADM1+) and a cDC2 (CD14-CD4-CD172a+CADM1+) subset ${ }^{20,21}$. pDCs are especially important for anti-viral responses, as they are the main producers of type I interferon (IFN)- $a^{22,23}$ and other cytokines, such as TNF and IL-12, especially after TLR7 and TLR9 stimulation in pigs ${ }^{20}$. $\mathrm{CDCs}$ are most efficient in presenting antigen and activating naïve T-cells by using MHC-class II molecules and costimulatory molecules, such as CD40, CD80 or CD86 ${ }^{21}$.

In this study, we focus on two synthetic TLR ligands that recently have proven to be efficient in stimulating the different adult porcine DC subsets: TLR2 ligand Pam3Cys and TLR 9 ligand CpG ODN, and could also be efficient in stimulating the porcine neonate DC subsets ${ }^{20}$. Auray et al. showed that TLR2 ligands were a potent activators of monocytes and DCs for pro-inflammatory responses in many monocytic cells, while TLR9 ligands typically induced strong IFN- $a$ and IL-12 responses in pDCs. With these ligands we therefore 
covered to different types of innate immune responses and ensured to have responses in all DC subsets and monocytes. First we identified the DC subsets and proportions of DC subsets and monocytes in the neonatal pig and compared these with adult pigs. Innate immune responses after TLR2 or TLR9 stimulation were compared between neonatal and adult pigs. DC activation was assessed by upregulation of cell-surface molecule MHCII and costimulatory molecule CD80/86, combined with intra-cellular staining for TNF. The same parameters were measured in monocytes. In the supernatant we measured the overall PBMC cytokine production and we evaluated the cytokine and TLR mRNA expression in an enriched mononuclear cell population.

\section{Materials and methods}

\section{Animals}

Blood was collected from four-day-old female piglets (neonates, $n=12$ ) or twelve-weekold female immunocompetent pigs (considered adults, $n=7$ ). All pigs were purchased from the same high health status pig farm in the Netherlands, reared under controlled conditions and were having the same genetic background. (Topigs Norsvin: Z-line (sow) $x$ Tempo line (boar)). The neonates were randomly selected from different sows and received sufficient colostrum. In total we used 12 neonates and 7 adults, as we were not able to perform all different assays on one animal due to small blood volume of the neonates. Pigs were euthanized with Euthasol ${ }^{\circ}$ and immediately exsanguinated. The blood was collected aseptically using $0.1 \%$ heparin (Heparine LEO, $5.000 \mathrm{I.E} / \mathrm{ml}$ ) as anticoagulant. All experiments were conducted in accordance to the Dutch animal experimental and ethical requirements and the project license application was approved by the Dutch Central Authority for Scientific Procedures on Animals (CCD) (Permit number: ADV401002015356).

\section{PBMC isolation and TLR specific stimulation}

Collected blood was diluted 1:1 with PBS containing 0.5 mM EDTA within 2 hours after collection and converted to a Leucosep ${ }^{\circledR}$ tube using a $60 \%$ FICOLL-PAQUE ${ }^{\mathrm{TM}}$ Plus densitygradient to isolate the PBMCs. Cells rested overnight at $4^{\circ} \mathrm{C}$ on ice and were plated in 12-well plates with $2.5 \times 106$ cells/well in $1 \mathrm{ml}$ RPMI 1640 medium (Gibco ${ }^{\circledR}$ ) supplemented with $10 \%$ fetal bovine serum and $1 \%$ Penicillin-Streptomycin (Gibco ${ }^{\circledR}$ ). Cells were stimulated with $10 \mu \mathrm{g} / \mathrm{ml}$ Pam3Cys-SKKKK (TLR2 ligand), (PAM3Cys L2000, EMC microcollections) or $5 \mu \mathrm{g} / \mathrm{ml} \mathrm{CpG}$ oligo-deoxynucleotide type A (TLR9 ligand) (CpG, sequence D32, ggTGCGTCGACGCAGggggg, Eurofins genomics), or they were left unstimulated as control. The TLR ligands from the same batch were diluted with PBS at the beginning of the study and stored in small aliquots of $100 \mathrm{ul}(1 \mathrm{mg} / \mathrm{ml})$ at $-20^{\circ} \mathrm{C}$. For each independent experiment new aliquots from the same batch were used to stimulate the samples. 


\section{PBMC Cytokine production measured by multiplex immunoassay}

PBMCs were stimulated for 7 hours with TLR2 or TLR9 ligand, or were left unstimulated as control. Supernatants of PBMC cultures were collected and frozen at $-80^{\circ} \mathrm{C}$ until analysis. Protein concentration of IFN-a, TNF, and IL- 6 in the supernatant were measured in duplicate with a custom-designed multiplex Cytometric Bead Array (PorcineProcartaplex ${ }^{\circledast}$; eBioscience) according to the manufacturer's instructions and read on a Luminex machine (Luminex ${ }^{\circledR} 200^{\mathrm{TM}}$ ). Cytokine concentrations were determined using XPONENT $^{\circledR}$ software. The detection limits of the cytokines were $0.72 \mathrm{pg} / \mathrm{ml}$ (IFN-a), $7.57 \mathrm{pg} / \mathrm{ml}$ (TNF) and 6.32 $\mathrm{pg} / \mathrm{ml}$ (IL-6), respectively.

\section{Cytokine and TLR mRNA expression in enriched innate mononuclear cell population}

The CD3- fraction was generated as an enriched innate mononuclear cell population. For CD3 depletion, stimulated PBMCs were first incubated with anti-porcine CD3 $\varepsilon$ antibody (clone PPT3, from SouthernBiotech) and then with anti-mouse lgG1 microbeads (Miltenyi Biotec). The CD3- fraction was then sorted using the Magnetic Activated Cell Sorting system (MACS ${ }^{\circledR}$, Miltenyi Biotec) with LD columns. Purity of the isolated cells was confirmed by flow cytometry ( $>95 \%$ purity). This CD3- fraction was harvested in TRIZOL ${ }^{\circledR}$ and stored at $-80^{\circ} \mathrm{C}$ until mRNA extraction was performed. mRNA was extracted by using the Directzol ${ }^{\circledR}$ RNA MiniPrep according to the manufacturer's instructions. mRNA quantity was accessed with the NanoDrop $1000^{\mathrm{TM}}$ (Thermo Fisher Scientific) by evaluating the optical density (OD) at $260 \mathrm{~nm}$ and the OD 260/280 ratio was then used to evaluate the quality.

The mRNA expression of TLR2 and TLR9, and the cytokines IFN- $\alpha, T N F$, IL- 6 and IL12p40 extracted from the PBMC/CD3- fraction was detected by RT-qPCR as previously described 24. Briefly, the SuperScript II Reverse Transcriptase (Invitrogen) was used to generate CDNA according to the manufacturer's instructions. CDNA was quantified by SYBR green incorporation by using the Applied Biosystems 7500/7500 standard ${ }^{\mathrm{TM}}$. All primer sequences were obtained from de Greeff et al. ${ }^{25}$.

Peptidyl-prolyl cis-trans isomerase $A(P P I A)$ was used for normalisation of the results given the stable expression in adult and neonatal PBMC/CD3 fraction. Quantification was done using serial dilutions of a plasmid with the gene of interest, which were used as internal standards. The efficiency of the PCR reaction was $90-100 \%$ for all reactions .

\section{DC subsets identification by flow cytometry}

After stimulation, PBMCs were harvested and resuspended in staining buffer (PBS containing $0.5 \mathrm{mM}$ EDTA and $0.25 \% \mathrm{BSA}$ ) and stained with antibodies (Abs) for DC subset identification as described previously ${ }^{21}$. Briefly, PBMCs were first incubated with antiCD172a (clone 74-22-15A from KINGFISHER) and anti-CADM1 (clone 3E1 from MBL) Abs. Cells were then incubated with the corresponding secondary Abs goat anti-mouse lgG2b PE (SouthernBiotec) and biotin-conjugated goat anti-chicken IgY (JacksonlmmunoResearch) 
respectively. Ig blocking was then performed with whole mouse IgG molecules (JacksonImmunoResearch). Finally, cells were incubated with the following conjugated Abs: anti-CD14-FITC (clone MIL2 from Abd Serotec) and anti-CD4-PerCP-Cy5.5 ${ }^{\text {TM }}$ (clone 74-12-4 from BD Bioscience) and V500-coupled streptavidin (BD Bioscience). PBMCs were then run on a FACSCANTO ${ }^{\text {TM }}$ (BD Biosciences) using the BD FACSDiva ${ }^{T M}$ software. The flow cytometry data were analysed with the $\mathrm{Flowjo}^{\mathrm{TM}}$ software version 10.0 .

\section{Assessment of cell-surface markers and intracellular TNF staining in DC subsets and monocytes by flow cytometry}

For the assessment of the cell-surface markers and intracellular TNF staining, PBMCs were harvested 5 hours after stimulation. Cell were then stained as described before for DC subsets and to assess the cell surface molecule expression, anti-CD80/86 (antiCD152 lg Fusion protein, clone BHK from Ancell) or anti-MHCII (anti-SLA class II DQ, clone K274.3G8 from AbD Serotec) were added in the first step of the DC subset staining. In the second step the corresponding secondary $A b$ was added: goat anti-mouse IgG2a secondary Ab AlexaFluor ${ }^{\circledast}$ (Invitrogen) for CD80/86 or goat anti-mouse IgG1 secondary Ab AlexaFluor ${ }^{\circledast}$ (Invitrogen) for MHCII.

For the intracellular staining, Brefeldin A ( $1 \mathrm{ng} / \mathrm{ml}$ eBioscience) was added one hour after stimulation to the cultures to stop the cytokine secretion. Cells were further cultured for 4 hours before being harvested for flow cytometry staining. For the intracellular staining, the cells were first stained with the Abs for the DC subsets as described previously and after this fixed in $4 \%$ paraformaldehyde. After a wash with $0.1 \%$ saponin (Panreac Applichem), cells were incubated with anti-TNF AlexaFluor ${ }^{\otimes} 647$ (clone Mab11 from BioLegend) in $0.3 \%$ saponin followed by another $0.1 \%$ saponin wash.

\section{Statistical analysis}

All statistics were calculated in GraphPad Prism 7.02 software. The two-way ANOVA followed by a Tukey's multiple comparisons test was used to assess significance for flow cytometry surface molecule expression and intracellular cytokine production in the DC subsets and the cytokine protein production of the PBMCs. The non-parametric MannWhitney $\mathrm{U}$ test was used to assess statistical significance of the RT-qPCR data and to evaluate the proportions of the DC subsets and monocytes $\left({ }^{* *} p<0.001,{ }^{* *} p<0.01\right.$ and $\left.{ }^{*} p<0.05\right)$.

\section{Results}

\section{Blood collection, PBMC isolation and number of animals}

From 12 neonates an average volume of $62 \pm 14 \mathrm{ml}$ blood was collected and subsequently $42 \pm 29 \times 10^{6}$ PBMCs were isolated. Due to this limited and variable amount of PBMCs more than one neonate was used to perform the different assays (flow cytometry, cytokine production and mRNA expression). From the 7 adult animals we collected sufficient blood 
and PBMCs (average of $120 \mathrm{ml}$ blood with $200 \pm 100 \times 10^{6}$ PBMCs). For the identification of the DC subsets and proportions by flow cytometry we used 6 animals from each age-group. For flow cytometry analysis for DC maturation and cytokine production measurement 4 animals from each age-group were used. For the mRNA expression (cytokines and TLRs) we used the data of 7 neonates and 4-5 adults. All analysis were performed in duplicate, which showed a high correlation $\left(R^{2}=0.96 \pm 0.05\right)$.

\section{PBMC cytokine production following TLR stimulation}

To investigate the impact of the neonatal and adult environment on monocytic cell and DC responses, we evaluated the cytokine production capacity of the whole PBMC population after TLR2 (Pam3Cys) and TLR9 (CpG) stimulation. Stimulation of PBMCs with CpG induced a significant increase of IFN- $a$, TNF and IL- 6 production in PBMCs of adults and a significant increase in IFN- $a$ production in neonates, with IFN- $a$ being the most abundant cytokine produced when compared to TNF and IL-6. IFN- $a$ and TNF production was significantly higher in the adult group compared to the neonates after $\mathrm{CpG}$ stimulation (Figs.1A and B).

PBMC stimulation with Pam3Cys induced a similar increase in IL-6, but not IFN-a production compared to unstimulated control PBMCs in all animals of both age groups (Fig. 1C). There was only one adult and one neonate that showed a significant higher level of TNF production from Pam3Cys stimulated PBMC compared to the average control (Fig. 1B).
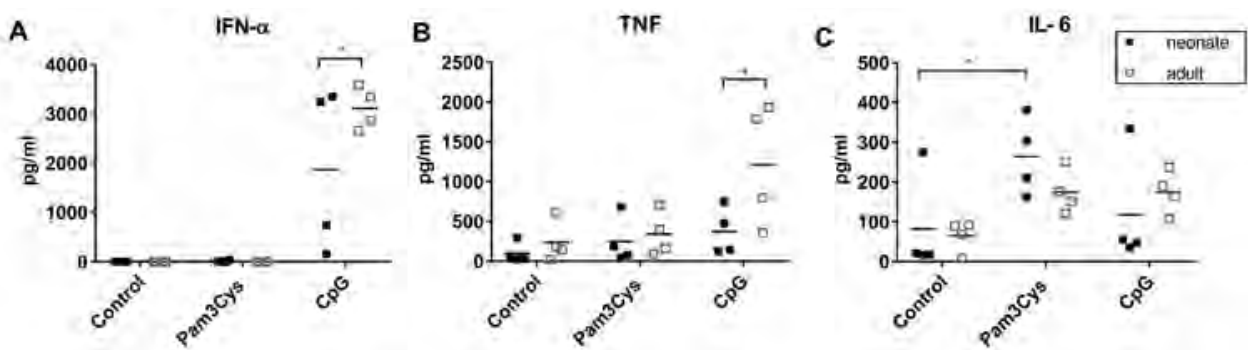

Figure 1: PBMC cytokine production following TLR stimulation.

PBMCs of neonates $(\square)$ and adults $(\square)$ were stimulated for 7 hours with $10 \mu \mathrm{g} / \mathrm{ml}$ Pam3Cys or $5 \mu \mathrm{g} / \mathrm{ml}$ $\mathrm{CpG}$ or were left unstimulated as control. INF-a, TNF, and IL- 6 production in $\mathrm{pg} / \mathrm{ml}$ are presented from neonates and adults $(n=4)$. Each symbol represents the average of the duplicate from one animal and the mean is shown for each data set. The results shown are from 4 independent experiments, which were performed on different days. Statistical significance was calculated using a two-way ANOVA followed by a Tukey's multiple comparisons test. ( ${ }^{* *} p<0.01,{ }^{*} p<0.05$ ). 


\section{Cytokine mRNA expression in enriched mononuclear cells following TLR stimulation}

The PBMC/CD3 fraction was generated to represent an enriched innate mononuclear cell population by removing the lymphocytes. Only $\mathrm{CpG}$ induced a clear upregulation (fold change) of mRNA IFN- $a$ expression in both age groups with no significant difference between the adults and the neonates (Fig. 2A). Pam3Cys and CpG induced upregulation of TNF and IL-6 mRNA expression, but there was no significant difference in upregulation between the age groups (Figs. 2B and C). CpG induced only in neonates a significant upregulation of IL-12p40 mRNA expression (Fig. 2D).
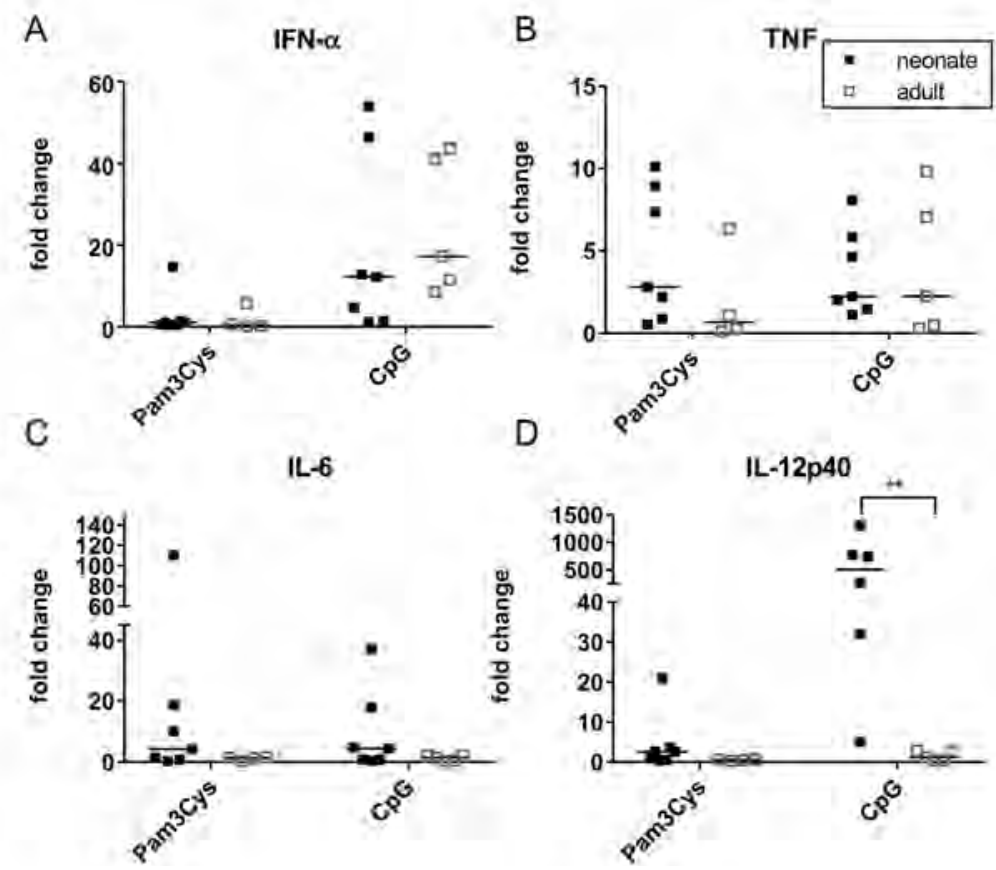

\section{Figure 2: Cytokine mRNA expression in enriched mononuclear cells following TLR} stimulation.

PBMCs of neonates ( $\square$ ) and adults ( $\square$ ) were stimulated for 7 hours with $10 \mu \mathrm{g} / \mathrm{ml} \mathrm{Pam3Cys} \mathrm{or} 5 \mu \mathrm{g} /$ $\mathrm{ml} \mathrm{CpG}$ or were left unstimulated as control. The fold change of IFN- $\mathrm{a}(\mathbf{A}), T N F(\mathbf{B}), I L-6(\mathbf{C})$ and IL12p40 (D) expression in PBMC/CD3- fraction is shown in the Pam3Cys and in the $\mathrm{CpG}$ stimulated animals. Each symbol represents one animal and the median is shown for each data set. Cytokine expression was assessed with RT-qPCR using PPIA expression to normalize the data. The results shown are from 6 independent experiments (neonates $n=7$ and adults $n=4-5$ ), which were performed on different days. Statistical significance was calculated using a Mann- Whitney U test, $\left({ }^{* *} p<0.01,{ }^{*} p<0.05\right)$. 


\section{TLR mRNA expression in enriched mononuclear cells following TLR stimulation}

To investigate if any differences observed between neonates and adults were related to TLR mRNA expression, we assessed this expression in the enriched mononuclear cells (PBMC/CD3- fraction). Basal TLR2 mRNA expression in the control PBMC/CD3- fraction showed no significant difference between neonates and adults and was not induced by the TLR ligands in any age group. Nevertheless, TLR2 mRNA expression was significantly higher in neonates compared to adults after Pam3Cys and CpG stimulation (Fig. 3A).

Basal TLR9 mRNA expression also showed no age-dependent difference in the control PBMC/CD3-fraction. However, only adult TLR9 mRNA expression showed a trend (although not significant) for downregulation after Pam3Cys and CpG stimulation and there was a significant higher expression for neonates after CpG stimulation (Fig. 3B).

A

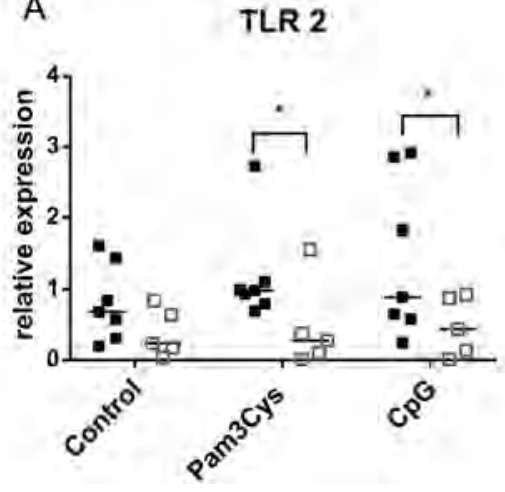

B

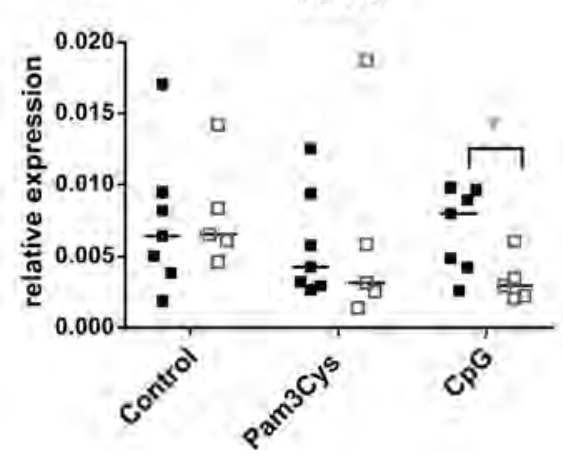

Figure 3: TLR mRNA expression in enriched mononuclear cells following TLR stimulation.

PBMCs of neonates ( $\square$ ) and adults ( $\square$ ) were stimulated for 7 hours with $10 \mu \mathrm{g} / \mathrm{ml} \mathrm{Pam3Cys} \mathrm{or} 5 \mu \mathrm{g} /$ $\mathrm{ml} \mathrm{CpG}$ or were left unstimulated as control. The relative expression of TLR2 (A) and TLR9 (B) in PBMC/CD3- cells is shown in the unstimulated control samples (basal expression), in the Pam3Cys and in the $\mathrm{CpG}$ stimulated animals. Each symbol represents one animal and the median is shown for the data set. Expression of TLR2 and TLR9 was assessed with RT-qPCR using PPIA expression to normalize the data. The results shown are from 6 independent experiments (neonates $n=7$ and adults $n=5$ ), which were performed on different days. Statistical significance was calculated using a Mann-Whitney $U$ test $\left({ }^{*} p<0.05\right)$.

\section{Identification of DC subsets in neonate and adult PBMCs}

The phenotype of the different DC subsets and monocytes in adults were determined by flow cytometry as previously described ${ }^{20}$ (Fig. 4A). After doublet discrimination, gates were set on cells with high forward and side scatter (big cells; excluding the lymphocytes) and the four surface markers CD14, CD172a, CADM1 and CD4 were used to identify the different $D C$ subsets. The $C D 14^{+}$cells were monocytes whereas $\mathrm{PDCs}$ were CD14- 
$\mathrm{CD}^{+}{ }^{+} \mathrm{CD} 172 \mathrm{a}^{+} \mathrm{CADM1} 1^{-}$, $\mathrm{CDC} 1$ were $\mathrm{CD} 14^{-} \mathrm{CD} 4^{-} \mathrm{CD} 172 \mathrm{a}^{\text {low }} \mathrm{CADM} 1^{+}$; and $\mathrm{CDC} 2$ were $\mathrm{CD} 14$ CD4 ${ }^{-} \mathrm{CD} 172 \mathrm{a}^{+} \mathrm{CADM1}{ }^{+}$. The same markers were used to identify these subsets in PBMCs isolated from neonatal pigs (Fig. 4B). The neonatal DC subsets presented similar levels of these markers compared to their adult counterparts. To investigate the possible difference in proportions of the $\mathrm{DC}$ subsets in neonates and adults, the percentage of pDCs, $\mathrm{CDC} 1$ and $\mathrm{CDC} 2$ cells in the whole PBMC population was calculated. Compared to monocytes these $D C$ frequencies were very low $(<0.5 \%)$ and there were no significance differences in the proportion of the different DC subsets in neonates compared to adults (Figs. 4C-E). However, the neonatal PBMCs contained significantly more monocytes (median $17.18 \%$ ) compared to the adult PBMCs (median 6.75\% monocytes in PBMCs) (Fig. 4F).
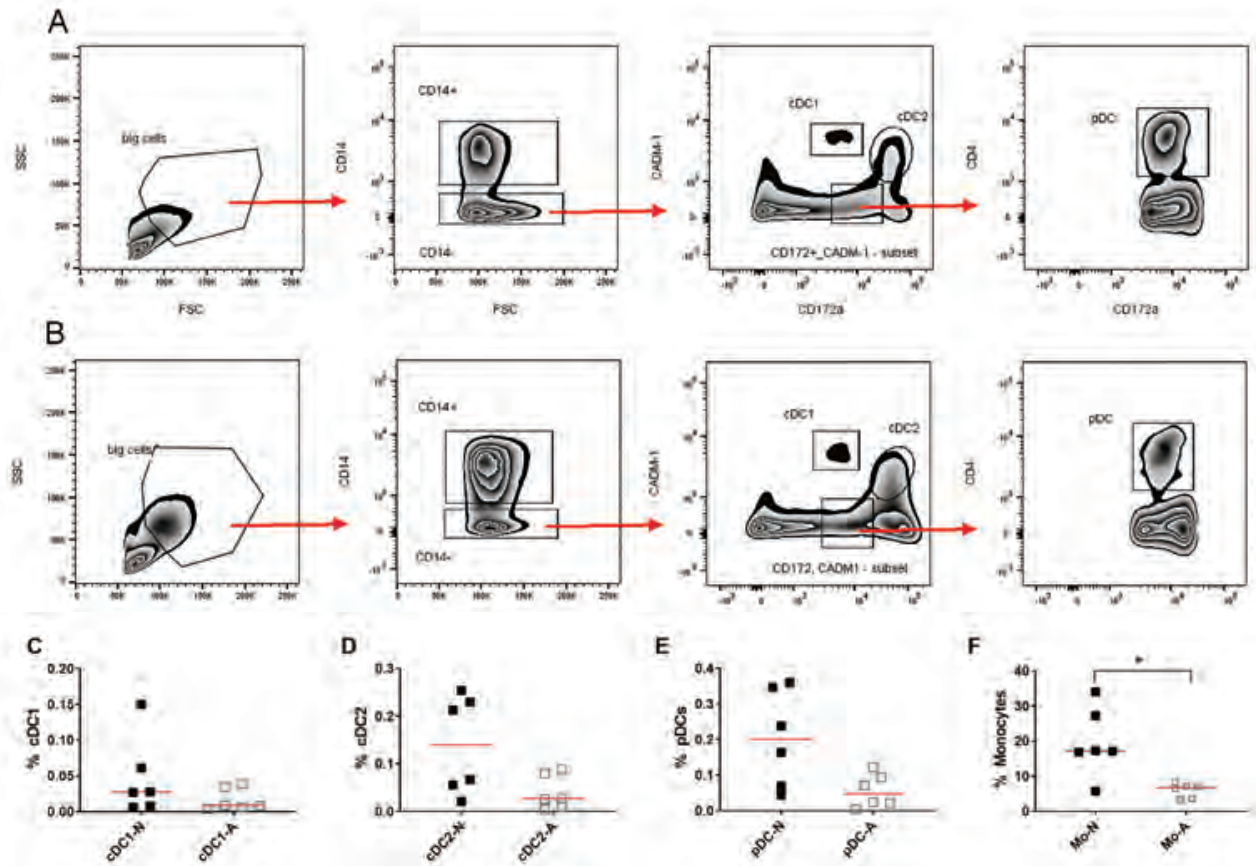

$\mathbf{F}$

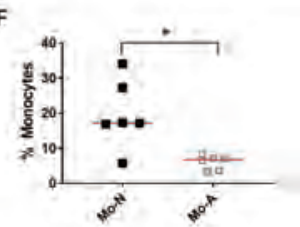

Figure 4: Phenotype and proportions of DC subsets and monocytes.

Gating strategy following five-colour flow cytometry by using antibodies against CD14, CD172a, CADM1 and CD4 in adult (A) and neonatal (B) PBMCs. After doublet discrimination, gates were set on cells with high forward and side scatter (big cells). Monocytes were defined as CD14+ cells and

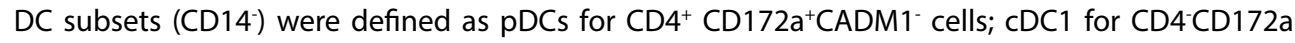
${ }^{\text {low }} \mathrm{CADM} 1^{+}$cells; $\mathrm{CDC} 2$ for $\mathrm{CD} 172 \mathrm{a}^{+} \mathrm{CADM} 1^{+}$cells.

The proportions of the different DC subsets (C-E) and monocytes (F) in neonatal ( $\mathbf{})$ and adult ( $\square$ ) PBMCs were determined in duplicate, each symbol represents the average of two measurement from one individual animal and the median is shown for each data set. The results shown are from 4 independent experiments $(n=6)$, which were performed on different days. Statistical significance was calculated using a Mann-Whitney U test, $\left({ }^{*} p<0.05\right)$. 


\section{CD80/86 and MHCIl expression in DC subsets and monocytes following TLR stimulation}

The maturation of the different DC subsets after specific TLR stimulation was investigated by the expression of the cell-surface molecule MHCII and costimulatory molecules CD80 and CD86 in PBMCs of neonates and adults. Monocytes were assessed for the same parameters as the DC subsets. Cell-surface expression of CD80/86 was most prominent in the neonatal and adult $\mathrm{CDC} 1$ and $\mathrm{CDC} 2$ subset and a lower expression was found in the pDCs and monocytes (Figs. 5A and B). Only in the adult cDC1 and cDC2 subsets, CpG induced a significant CD80/86 upregulation (Figs 5A and B). For Pam3Cys (TLR2), there was a significant CD80/86 upregulation in neonates and adults for the CDC1 subset, where the neonatal $\mathrm{CDC} 1$ were more responsive to Pam3Cys stimulation compared to adults age groups (Figure 5C). The adults showed also a CD80/86 upregulation in the CDC2 subset after Pam3Cys stimulation. MHCIl expression was observed in $\mathrm{CDC1}, \mathrm{CDC} 2$ and $\mathrm{pDC}$ subset in the neonatal and adult PBMCs with no difference between unstimulated control and TLR specific stimulated samples (Figs. 5D and E). The neonate CDC1 subset showed a higher expression of $\mathrm{MHCll}$ in the control samples compared to adults (Fig. 5F).
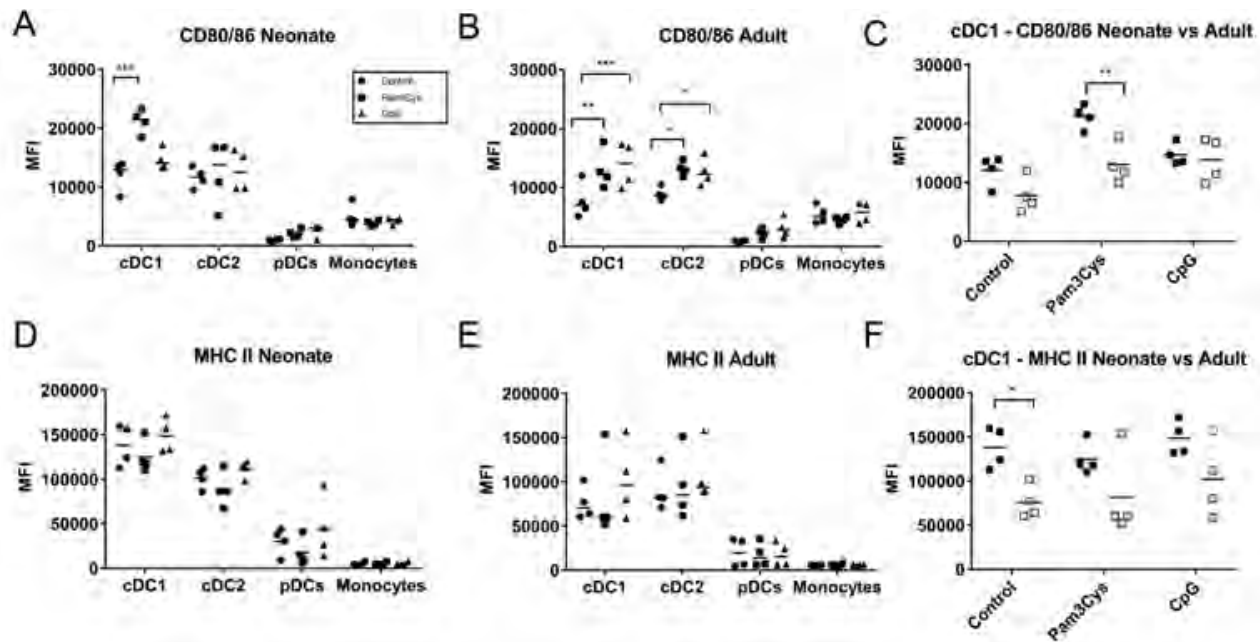

\section{Figure 5: CD80/86 and MHCIl expression in DC subsets and monocytes following TLR stimulation.}

Whole PBMCs were stimulated for $5 \mathrm{~h}$ with $10 \mathrm{ug} / \mathrm{ml}$ Pam3Cys or $5 \mathrm{ug} / \mathrm{ml} \mathrm{CpG} \mathrm{or} \mathrm{left} \mathrm{unstimulated}$ as control. The mean fluorescence intensity (MFI) for CD80/86 and MHCIl expression was measured in duplicate for neonates ( $A$ and $D)(n=4)$ and adults $(B$ and $E)(n=4)$, each symbol represents the average of two measurement from one individual animal and the mean is shown for each data set. For the CDC1 subset the MFI for CD80/86 (C) and MHC II (F) was compared between neonates ( $\mathbf{\square})$ and adults( $\square)$. The results shown are from 4 independent experiments, which were performed on different days. Statistical significance was calculated using a two-way ANOVA followed by a Tukey's multiple comparisons test. $\left({ }^{* * *} p<0.001,{ }^{* *} p<0.01,{ }^{*} p<0.05\right)$. 
A

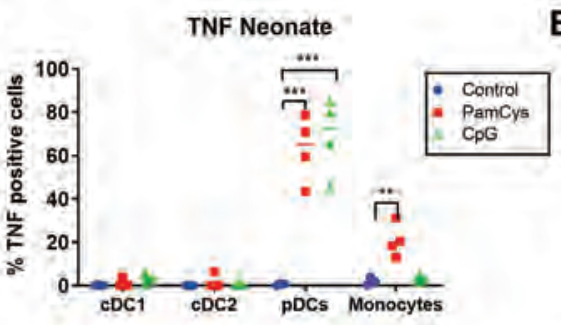

C

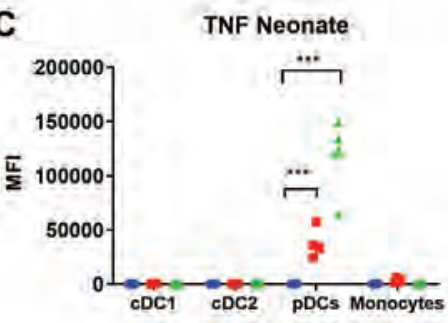

E

$\mathbf{G}$

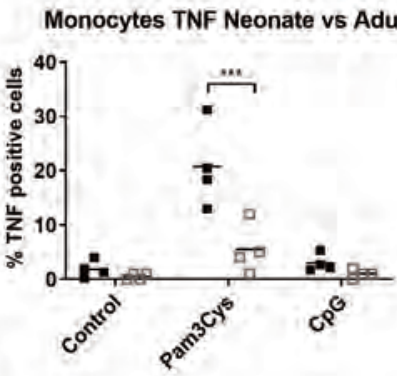

pDC TNF Neonate vs Adult

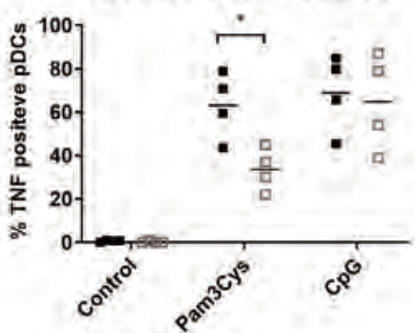

B

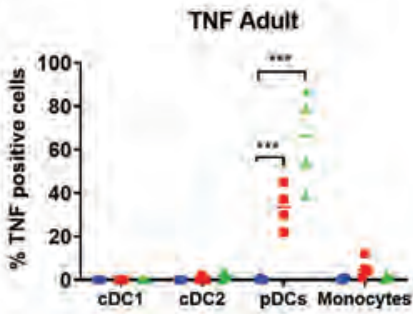

D

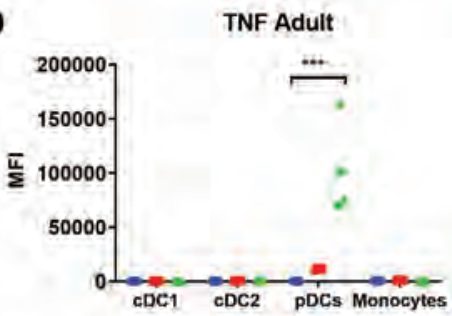

$\mathbf{F}$

H
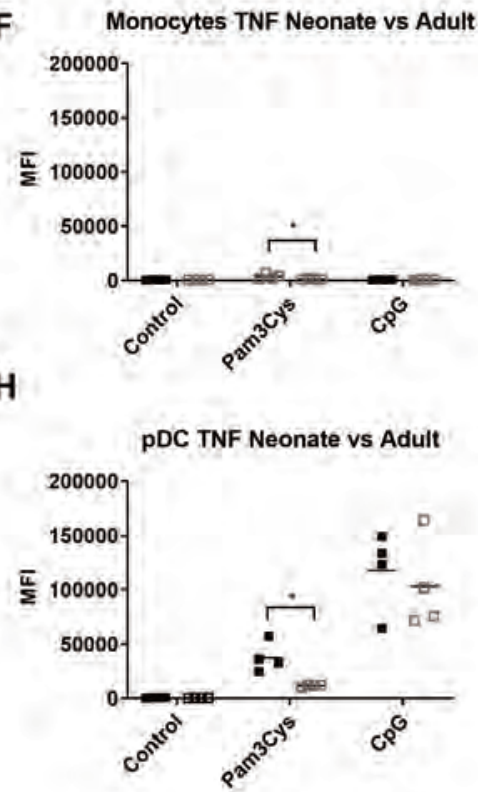

Figure 6: Intracellular TNF expression in DCs subsets following TLR stimulation.

Whole PBMCs were stimulated with $10 \mathrm{ug} / \mathrm{ml}$ Pam3Cys or $5 \mathrm{ug} / \mathrm{ml} \mathrm{CpG}$ or left unstimulated as control. Results are expressed either as the percentage of TNF positive cells (A,B,E and $\mathbf{G})$ or as the mean fluorescence intensity (MFI) of the cell subset (C, D, $\mathbf{F}$ and $\mathbf{H})$. The TNF was measured in duplicate for neonates $(n=4)$ and adults $(n=4)$, each symbol represents the average of the duplicate from one animal and the mean is shown for each data set. The TNF response of the pDCs (E and F) and monocytes (G and $\mathbf{H}$ ) was compared between neonates ( $\square$ ) and adults( $\square$ ). The results shown are from 4 independent experiments, which were performed on different days. Statistical significance was calculated using a two-way ANOVA followed by a Tukey's multiple comparisons test. $\left({ }^{* * *} p<0.001,{ }^{* *} p<0.01,{ }^{*} p<0.05\right)$. 


\section{TNF expression in DC subsets and monocytes following TLR stimulation}

Another characteristic of activated DCs is their capacity to produce cytokines. We stimulated PBMCs and performed intra-cellular flow cytometry staining to assess TNF production of the different DC subsets and monocytes. Following TLR2 stimulation, pDCs and monocytes were the only subsets to produce TNF in both age groups. pDCs were the only cell type responding to CpG. In addition, stimulated pDC clearly had the highest percentage of TNF expressing cells as well as TNF mean fluorescence intensity (MFI, reflecting the amount of cytokine) (Figs. 6A-D).

When comparing the responses of neonates with adults we found that Pam3Cys, but not CpG induced a significant higher percentage of TNF expressing pDCs as well as TNF $\mathrm{MFI}$ in neonates (Fig. 6E and F). Interestingly, all neonates tested showed a significant TNF response in monocytes after Pam3Cys stimulation, while monocytes from adult pigs generally not responded (Figs. 6G and $\mathrm{H}$ ).

\section{Discussion}

The innate immune response was investigated in mononuclear cells of four-day-old neonatal pigs who received sufficient colostrum and were reared under the same conditions with the same genetic background as the adult pigs in this study. We aimed to identify neonatal responsiveness after specific TLR stimulation to explore strategies for optimisation of neonatal responses to vaccination. To this end, we decided to stimulate TLR2 and TLR9 with synthetic ligands in neonates, which have proven to be effective in adult porcine blood DC subsets: TLR2 ligand Pam3Cys and TLR 9 ligand CpG ODN ${ }^{20}$. TLR2 targeting as adjuvant gave promising, yet contradictory results for vaccine application in mice and humans ${ }^{26}$. TLR9 stimulation generally induces a Th1 biased immune response ${ }^{27}$, which could be beneficial to redirect the Th2 skewed response in neonates.

Using whole PBMCs or enriched mononuclear cells (PBMC/CD3- fraction), we found that Pam3Cys induced similar levels of IL-6 and TNF, but no IFN-a production in both age groups relating to previous observations ${ }^{20}$. Nevertheless, neonates showed a consistent trend, albeit not significant, of producing more IL-6 and expressing more IL-6 mRNA after Pam3Cys stimulation compared to adults, which was also found in moDCs derived from human cord blood monocytes ${ }^{28}$. Together with the corresponding TNF response in adults and neonates, this suggest that the TLR2 mediated immune response in neonatal PBMCs is at least at an adult level.

Whereas CpG stimulation induced IFN- $a$ and TNF production in neonates and adults, only the neonates showed a significant increase of IL12p40 mRNA expression in the enriched mononuclear cells after CpG stimulation. This higher IL-12p40 mRNA expression in neonates was described before in pigs ${ }^{29}$ and goats ${ }^{30}$. Auray et al. ${ }^{20}$ have shown that only pDCs produce IL12p40 and that this is associated with IL12p35 expression, which would suggest that the IL 12p40 expression measured in the present study reflects IL12 production. For IFN-a we found an equal mRNA expression level of the enriched mononuclear cells in 
adults and neonates, indicating that neonates are able to upregulate the expression of this cytokine after CpG stimulation as found in other studies on cord blood for neonatal horses ${ }^{31}$ and humans ${ }^{17}$. Together, this clear IFN- $a$ - and IL12p40 response would reflect the ability of CpG to induce a Th1 type cytokine response in porcine neonates.

To investigate cell-specific differences, we focused on the innate immune cells of the mononuclear phagocyte system: $\mathrm{CDC} 1, \mathrm{CDC} 2, \mathrm{pDCs}$ and monocytes. The present study demonstrated that neonates have similar DC subsets and proportion of pDCs, CDC1 and CDC2 in the total PBMC population as adult animals, although we noticed larger individual variation in the subsets of the neonates compared to adults. Our results are in agreement with an earlier study demonstrating this for pDCs in pigs ${ }^{29}$. The larger proportion of monocytes found in the neonatal PBMCs compared to adults was also found in human studies with cord blood ${ }^{32,33}$ and could reflect a higher rate of monocytopoiesis in the bone marrow. We also investigated expression of cell-surface markers on DC subsets at steadystate. In our study the porcine neonates expressed higher levels of MHC II on CDC1 and no differences were seen concerning the levels of the costimulatory molecules CD80/86. In contrast, human studies have demonstrated lower steady-state expression of MHCII and CD80/86 on APC in neonates ${ }^{34}$, but these studies used foetal cord blood, which is different from the neonatal blood of four-day-old piglets we used in our study.

The ability of neonatal porcine DCs to get efficiently activated was measured and compared to adults by the expression of costimulatory molecules and cytokine production. As already shown in adult pigs, neonatal pDCs were the main producers of TNF ${ }^{20}$. In neonates TLR2 stimulation with Pam3Cys induced a stronger TNF expression in pDCs, and upregulation of CD80/86 in CDC1 compared to what was observed in adult cells. These results suggest an increased maturation capacity of porcine neonatal DCs after Pam3Cys stimulation compared to adults. Studies on human cord blood DCs ${ }^{28}$, horse PBMCs ${ }^{31}$ and human naïve T-cells ${ }^{35}$ have also reported this potency of TLR2 stimulation in neonates for vaccination. A remarkable finding was that only after Pam3Cys stimulation neonatal porcine monocytes responded with high numbers of TNF expressing cells. This response was not observed in adult porcine monocytes as demonstrated by Auray et al. ${ }^{20}$. However, in humans, both neonatal and adult monocytes showed increased TNF expression after Pam3Cys stimulation ${ }^{36}$ suggesting development-related species differences for Pam3Cys stimulation.

After TLR9 stimulation with CPG the different DC subsets of neonates and adults showed an equivalent response in terms of TNF production by pDCs and CD80/86 expression by $\mathrm{CDC1}$, demonstrating that in vitro $\mathrm{DCs}$ of neonates are able to mount an adult-like response ${ }^{37}$. Auray et al. (2013) showed that porcine neonatal BDCs after CpG stimulation displayed a more mature phenotype (CD80/86 expression) compared to adults, while other studies in human and horse cord blood ${ }^{15,17}$ found a lower responsiveness after $\mathrm{CpG}$ stimulation in neonates compared to adults. These differences in upregulation of CD80/86 between studies could be a result of disparities between age-groups, 
experimental conditions, DC populations and species, which complicates the comparison of neonate in vitro experiments, especially where DCs are involved ${ }^{38,39}$.

Finally, we investigated the TLR2 and TLR9 expression in the enriched mononuclear cells to see if any difference in response between neonates and adults could be correlated to differences in TLR expression. At steady-state we found no significant difference in TLR2 and TLR9 mRNA expression between neonates and adults in the PBMC/CD3fraction, as previously shown for TLR9 expression in porcine monocytes ${ }^{29}$. This suggest that microenvironment and downstream NF- $\beta \beta$ activation play an important role in this differential responsiveness between neonates and adults after specific TLR stimulation as suggested before in other studies ${ }^{40,41}$. Nonetheless, after stimulation we found agedependent differences, which were most likely caused by the not significant trend of neonatal upregulation of TLR2 expression and downregulation of adult TLR9 expression in our study. Another study in neonatal and adult horses has also demonstrated this upregulation of TLR2 expression and downregulation of TLR9 expression after Pam3Cys stimulation ${ }^{31}$. Further research is needed to elucidate the mechanism of this agedependent response in porcine DCs and monocytes after TLR2 stimulation.

Transcribing our results to possible adjuvant application, TLR2 stimulation with Pam3Cys provided in neonates a stronger activation of DCs and monocytes compared to adult pigs. Nonetheless, this neonatal Pam3Cys response was without IFN-a and IL-12p40 induction and with a higher II-6 response compared to $\mathrm{CpG}$ and by this not necessarily Th1 directed. TLR9 stimulation with CpG generated a distinct Th1 skewed response, in neonates as found by other studies in goats ${ }^{16}$ and humans ${ }^{42,43}$. CpG administration has induced in vivo also a Th1 skewed response in neonatal piglets ${ }^{44}$, supporting the application as adjuvant in neonatal vaccine research.

Because both TLRs showed different properties, co-stimulation of TLR2 and TLR9 could be an interesting option, as suggested by other studies for different TLRs ${ }^{45-47}$.

Individual variation, as commonly seen in outbred animals and a small blood volume in neonates reducing the number of assays per animal are potential limitations of our study. However, our consistent in vitro findings, despite the individual variation, contribute to the further understanding of the porcine neonate immune response. Our data demonstrate that porcine neonates comprised the same DC subsets and proportions and are not generally incompetent in responding to TLR2 and TLR9 ligands. However, the DC response in our study cannot explain why neonates are generally less immunocompetent when compared to adults and it cannot be concluded that DC or APC functionality is not of importance for this difference. For instance, it could be that other functions of the APCs are less potent in neonates or that the frequency of the DCs in neonatal lymphoid tissue is different. Next steps could be to have a closer look at APCs in the lymphoid tissue combined with a focus on the lymphoid structure and stromal components.

We also have to be aware that the in vitro selection process cannot entirely reflect the complex interactions occurring in vivo, because of the lack of the essential 
microenvironment (neonatal plasma and the organs and tissues essential in the immune system). This is especially true for the $\mathrm{pDC}$ fraction in neonates resulting in different outcomes for comparable in vivo experiments ${ }^{48}$. So caution is needed for projection of our results to possible in vivo applications.

\section{Conclusion}

We showed that the porcine neonate PBMCs comprised the same DC subsets and similar proportions and a higher proportion of monocytes, as their adult counterparts. Porcine neonate $\mathrm{pDCs}, \mathrm{CDC} 1$ and monocytes were more responsive after TLR2 stimulation with respect to TNF expression ( $\mathrm{pDC}$ and monocytes) and CD80/86 upregulation (cDC1). Furthermore TLR9 stimulation induced increased IL12p40 mRNA expression only in neonate mononuclear cells. Overall, our results indicate responsiveness of neonates after TLR2 and TLR9 stimulation with age-dependent differences in porcine innate immune response, also suggesting that $\mathrm{CpG}$ and Pam3Cys could be interesting components to stimulate the neonate innate immune system for future vaccine research.

\section{Acknowledgments}

We thank Ben Meijer for assistance with the flow cytometry. This project has received funding from the European Union's Horizon 2020 Programme for research, technological development and demonstration under the Grant Agreement $n^{\circ} 633184$. This publication reflects the views only of the author, and not the European Commission (EC). The EC is not liable for any use that may be made of the information contained herein. 


\section{References}

1 Rose, N. \& Andraud, M. The use of vaccines to control pathogen spread in pig populations. Porcine health management 3, 8, doi:10.1186/s40813-017-0053-6 (2017).

2 Murtaugh, M. P. Advances in swine immunology help move vaccine technology forward. Veterinary immunology and immunopathology 159, 202-207, doi:10.1016/j.vetimm.2014.02.017 (2014).

3 Kollmann, T. R. \& Marchant, A. Towards Predicting Protective Vaccine Responses in the Very Young. Trends in immunology 37, 523-534, doi:10.1016/j.it.2016.05.005 (2016).

4 Mohr, E. \& Siegrist, C. A. Vaccination in early life: standing up to the challenges. Current opinion in immunology 41, 1-8, doi:10.1016/j.coi.2016.04.004 (2016).

5 Siegrist, C. A. Mechanisms by which maternal antibodies influence infant vaccine responses: review of hypotheses and definition of main determinants. Vaccine 21, 3406-3412 (2003).

6 Kumar, S. K. \& Bhat, B. V. Distinct mechanisms of the newborn innate immunity. Immunol Lett 173, 42-54, doi:10.1016/j.imlet.2016.03.009 (2016).

7 Levy, O. Innate immunity of the newborn: basic mechanisms and clinical correlates. Nature reviews. Immunology 7, 379-390, doi:10.1038/nri2075 (2007).

8 Kollmann, T. R. et al. Neonatal innate TLR-mediated responses are distinct from those of adults. Journal of immunology 183, 7150-7160, doi:10.4049/jimmunol.0901481 (2009).

9 Holt, P. G. \& Jones, C. A. The development of the immune system during pregnancy and early life. Allergy 55, 688-697 (2000).

10 Savelkoul, H. F., Ferro, V. A., Strioga, M. M. \& Schijns, V. E. Choice and Design of Adjuvants for Parenteral and Mucosal Vaccines. Vaccines 3, 148-171, doi:10.3390/vaccines3010148 (2015).

11 Toussi, D. N. \& Massari, P. Immune Adjuvant Effect of Molecularly-defined Toll-Like Receptor Ligands. Vaccines 2, 323-353, doi:10.3390/vaccines2020323 (2014).

12 Danis, B. et al. Interferon regulatory factor 7-mediated responses are defective in cord blood plasmacytoid dendritic cells. European journal of immunology 38, 507-517, doi:10.1002/eji.200737760 (2008).

13 Aksoy, E. et al. Interferon regulatory factor 3-dependent responses to lipopolysaccharide are selectively blunted in cord blood cells. Blood 109, 2887-2893, doi:10.1182/blood-2006-06-027862 (2007).

14 Angelone, D. F. et al. Innate immunity of the human newborn is polarized toward a high ratio of IL-6/TNFalpha production in vitro and in vivo. Pediatric research 60, 205-209, doi:10.1203/01.pdr.0000228319.10481. ea (2006).

15 Nguyen, M. et al. Acquisition of adult-like TLR4 and TLR9 responses during the first year of life. PloS one 5, e10407, doi:10.1371/journal.pone.0010407 (2010).

16 Tourais-Esteves, I., Bernardet, N., Lacroix-Lamande, S., Ferret-Bernard, S. \& Laurent, F. Neonatal goats display a stronger TH1-type cytokine response to TLR ligands than adults. Developmental and comparative immunology 32, 1231-1241, doi:10.1016/j.dci.2008.03.011 (2008).

17 Schuller, S. et al. The TLR-specific adjuvants R-848 and CpG-B endorse the immunological reaction of neonatal antigen-presenting cells. Pediatric research 80, 311-318, doi:10.1038/pr.2016.71 (2016).

18 Dutertre, C. A., Wang, L. F. \& Ginhoux, F. Aligning bona fide dendritic cell populations across species. Cell. Immunol. 291, 3-10, doi:10.1016/j.cellimm.2014.08.006 (2014). 
19 McCullough, K. C. \& Summerfield, A. Targeting the porcine immune system--particulate vaccines in the 21st century. Developmental and comparative immunology 33, 394-409, doi:10.1016/j.dci.2008.07.015 (2009).

20 Auray, G. et al. Characterization and Transcriptomic Analysis of Porcine Blood Conventional and Plasmacytoid Dendritic Cells Reveals Striking Species-Specific Differences. Journal of immunology 197, 4791-4806, doi:10.4049/jimmunol.1600672 (2016).

21 Summerfield, A., Auray, G. \& Ricklin, M. Comparative dendritic cell biology of veterinary mammals. Annual review of animal biosciences 3, 533-557, doi:10.1146/annurev-animal-022114-111009 (2015).

22 Summerfield, A. et al. Porcine peripheral blood dendritic cells and natural interferon-producing cells. Immunology 110, 440-449 (2003).

23 Reizis, B., Colonna, M., Trinchieri, G., Barrat, F. \& Gilliet, M. Plasmacytoid dendritic cells: one-trick ponies or workhorses of the immune system? Nature reviews. Immunology 11, 558-565, doi:10.1038/nri3027 (2011). Wichgers Schreur, P. J., Rebel, J. M., Smits, M. A., van Putten, J. P. \& Smith, H. E. Lgt processing is an essential step in Streptococcus suis lipoprotein mediated innate immune activation. PloS one 6, e22299, doi:10.1371/ journal.pone.0022299 (2011).

25 de Greeff, A. et al. Involvement of NF-kappaB and MAP-kinases in the transcriptional response of alveolar macrophages to Streptococcus suis. Veterinary microbiology 141, 59-67, doi:10.1016/j.vetmic.2009.07.031 (2010).

26 Basto, A. P. \& Leitao, A. Targeting TLR2 for vaccine development. Journal of immunology research 2014, 619410, doi:10.1155/2014/619410 (2014).

27 Scheiermann, J. \& Klinman, D. M. Clinical evaluation of CpG oligonucleotides as adjuvants for vaccines targeting infectious diseases and cancer. Vaccine 32, 6377-6389, doi:10.1016/j.vaccine.2014.06.065 (2014). Nohmi, K. et al. Zymosan Induces Immune Responses Comparable with Those of Adults in Monocytes, Dendritic Cells, and Monocyte-Derived Dendritic Cells from Cord Blood. J. Pediatr. 167, 155-162 e151-152, doi:10.1016/j.jpeds.2015.03.035 (2015).

29 Auray, G. et al. Porcine neonatal blood dendritic cells, but not monocytes, are more responsive to TLRs stimulation than their adult counterparts. PloS one 8, e59629, doi:10.1371/journal.pone.0059629 (2013).

30 Ferret-Bernard, S. et al. Mesenteric lymph node cells from neonates present a prominent IL-12 response to CpG oligodeoxynucleotide via an IL-15 feedback loop of amplification. Veterinary research 42, 19, doi:10.1186/1297-9716-42-19 (2011).

31 Vendrig, J. C., Coffeng, L. E. \& Fink-Gremmels, J. Effects of Separate and Concomitant TLR-2 and TLR4 Activation in Peripheral Blood Mononuclear Cells of Newborn and Adult Horses. PloS one 8, e66897, doi:10.1371/journal.pone.0066897 (2013).

32 Prabhu, S. B. et al. Comparison of Human Neonatal and Adult Blood Leukocyte Subset Composition Phenotypes. PloS one 11, e0162242, doi:10.1371/journal.pone.0162242 (2016).

33 Zenarruzabeitia, O. et al. The expression and function of human CD300 receptors on blood circulating mononuclear cells are distinct in neonates and adults. Scientific reports 6, 32693, doi:10.1038/srep32693 (2016).

34 Velilla, P. A., Rugeles, M. T. \& Chougnet, C. A. Defective antigen-presenting cell function in human neonates. Clinical immunology (Orlando, Fla.) 121, 251-259, doi:10.1016/j.clim.2006.08.010 (2006). 
35 Sinnott, B. D., Park, B., Boer, M. C., Lewinsohn, D. A. \& Lancioni, C. L. Direct TLR-2 Costimulation Unmasks the Proinflammatory Potential of Neonatal CD4+ T Cells. Journal of immunology 197, 68-77, doi:10.4049/ jimmunol.1501297 (2016).

36 Dreschers, S. et al. Reduced PICD in Monocytes Mounts Altered Neonate Immune Response to Candida albicans. PloS one 11, e0166648, doi:10.1371/journal.pone.0166648 (2016).

37 Zhang, X. et al. Neonatal plasmacytoid dendritic cells (pDCs) display subset variation but can elicit potent anti-viral innate responses. PloS one 8, e52003, doi:10.1371/journal.pone.0052003 (2013).

38 Auray, G. et al. Differential activation and maturation of two porcine DC populations following TLR ligand stimulation. Molecular immunology 47, 2103-2111, doi:10.1016/j.molimm.2010.03.016 (2010).

39 Facci, M. R. et al. A comparison between isolated blood dendritic cells and monocyte-derived dendritic cells in pigs. Immunology 129, 396-405, doi:10.1111/j.1365-2567.2009.03192.x (2010).

40 Kollmann, T. R., Levy, O., Montgomery, R. R. \& Goriely, S. Innate immune function by Toll-like receptors: distinct responses in newborns and the elderly. Immunity 37, 771-783, doi:10.1016/j.immuni.2012.10.014 (2012).

41 Shao, L., Fischer, D. D., Kandasamy, S., Saif, L. J. \& Vlasova, A. N. Tissue-specific mRNA expression profiles of porcine Toll-like receptors at different ages in germ-free and conventional pigs. Veterinary immunology and immunopathology 171, 7-16, doi:10.1016/j.vetimm.2016.01.008 (2016).

42 Gay, N. et al. Age-Dependent Maturation of Toll-Like Receptor-Mediated Cytokine Responses in Gambian Infants. PloS one 6, e18185, doi:10.1371/journal.pone.0018185 (2011).

43 Zhang, X. et al. Plasmacytoid dendritic cells engagement by influenza vaccine as a surrogate strategy for driving T-helper type 1 responses in human neonatal settings. The Journal of infectious diseases 210, 424434, doi:10.1093/infdis/jiu103 (2014).

44 Cai, H. et al. CpG oligodeoxynucleotide protect neonatal piglets from challenge with the enterotoxigenic $\mathrm{E}$. coli. Veterinary immunology and immunopathology 161, 66-76, doi:10.1016/j.vetimm.2014.07.003 (2014).

45 Sugitharini, V., Shahana, P., Prema, A. \& Berla Thangam, E. TLR2 and TLR4 co-activation utilizes distinct signaling pathways for the production of Th1/Th2/Th17 cytokines in neonatal immune cells. Cytokine 85, 191-200, doi:10.1016/j.cyto.2016.06.024 (2016).

46 van Haren, S. D. et al. Age-Specific Adjuvant Synergy: Dual TLR7/8 and Mincle Activation of Human Newborn Dendritic Cells Enables Th1 Polarization. Journal of immunology 197, 4413-4424, doi:10.4049/ jimmunol.1600282 (2016).

$47 \mathrm{Hu}$, Y. et al. Synergy of TLR3 and 7 ligands significantly enhances function of DCs to present inactivated PRRSV antigen through TRIF/MyD88-NF-kappaB signaling pathway. Scientific reports 6, 23977, doi:10.1038/ srep23977 (2016).

48 Belnoue, E. et al. Functional limitations of plasmacytoid dendritic cells limit type I interferon, T cell responses and virus control in early life. PloS one 8, e85302, doi:10.1371/journal.pone.0085302 (2013). 


\title{
Early immune responses in skin and lymph node after skin vaccination in neonatal and adult pigs
}

\author{
Sandra Vremann ${ }^{1,2}$, Johanna M.J. Rebel ${ }^{4}$, Joanne McCaffrey ${ }^{3}$, Kristina Ledl' \\ Ksenia Arkhipova', Damien Collins ${ }^{3}$, Dennis McDaid ${ }^{3}$, Huub F.J. Savelkoul', \\ Kerstin Skovgaard ${ }^{5}$, Anne C. Moore ${ }^{6}$ and Norbert Stockhofe-Zurwieden ${ }^{1}$
}

${ }^{1}$ Wageningen Bioveterinary Research, Wageningen University \& Research, P.O. Box 29703, 2502 LS The Hague, The Netherlands

${ }^{2}$ Cell Biology \& Immunology group, Wageningen University \& Research P.O. Box 338, 6700 HA Wageningen, The Netherlands

${ }^{3}$ Xeolas, Pharmaceuticals, Dublin Ireland

${ }^{4}$ Wageningen Livestock Research, Wageningen University \& Research, The Netherlands

${ }^{5}$ Department of Biotechnology and Biomedicine, Technical University of Denmark, Lyngby, Denmark

${ }^{6}$ School of Biochemistry and Cell Biology, School of Pharmacy University College Cork, Cork, Ireland 


\begin{abstract}
The skin is potentially an important vaccine delivery route facilitated by a high number of resident antigen presenting cells (APCs), are known to be stimulated by different Toll-like receptor agonists (TLRa). In this study adult and neonatal pigs were vaccinated in the skin using dissolving microneedle patches to investigate the immuno-stimulatory potential of different TLRa and possible age-dependent differences early after vaccination. These patches contained TLR1/2a (Pam3Cys), TLR7/8a (R848) or TLR9a (CpG ODN) combined with inactivated porcine reproductive and respiratory syndrome virus (PRRSV) or with an oil-in-water stable emulsion. Vaccinated skin and draining lymph nodes were analysed for immune response genes using microfluidic high-throughput qPCR to evaluate the early immune response and activation of APCs. Skin pathology and immunohistochemistry were used to evaluate the local immune responses and APCs in the vaccinated skin respectively.

In both neonatal and adult pigs, skin vaccination with TLR7/8a induced the most prominent early inflammatory and immune cell responses, particularly in the skin. Skin histopathology and immunohistochemistry of APCs showed comparable results for neonatal and adult pigs after vaccination with the different TLRa vaccines. However, in vaccinated neonatal pigs in the skin and draining lymph node more immune response related genes were upregulated compared to adult pigs. We showed that both neonatal and adult skin could be stimulated to develop an immune response, particularly after TLR7/8a vaccination, with age-dependent differences in regulation of immune genes. Therefore, age-dependent differences in local early immune responses should be considered when developing skin vaccines.
\end{abstract}




\section{Introduction}

The skin is the largest organ in the body and forms a physical and immunological boundary to protect from injury and pathogens ${ }^{1,2}$. Because of its immunological role the skin is an attractive delivery route for vaccination. Skin vaccination can induce immune responses quantitatively equivalent or qualitatively enhanced compared to conventional intramuscular (i.m.) vaccination ${ }^{3,4}$, sometimes with a reduction of the vaccine antigendose ${ }^{5,6}$. The epidermis and dermis are the most important layers that determine the immune responses within the skin ${ }^{7,8}$. These layers can be physically breached by dissolving microneedle (DMN)-patches, a needle-free delivery system for skin vaccination ${ }^{9}$, also termed "microarray patches". DMN-patches can target the high number of APCs present in the both the epidermis and dermis, which reside in a quiescent/immature state in the skin. After vaccination, activated APCs mature and transport the vaccine-antigen to the draining lymph nodes (LNs), where the antigen is presented to naïve T cells ${ }^{10,11}$ to induce an immune response. Besides APCs, epidermal keratinocytes and newly recruited dermal immune cells also contribute to this skin vaccinated induced immune response ${ }^{11,12}$.

Dendritic cells (DCs) are professional APCs, which are essential to induce effective immune responses after vaccination ${ }^{13,14}$. We studied two different DC subsets in the skin of adult and neonatal pigs: Langerhans cells (LC) in the epidermis and dermal DCs $(\mathrm{dDC})$ in the dermis, by detecting the expression of cell-surface markers (CD1a' MHCIl and $\mathrm{CD} 163)^{7,15,16}$ combined with their localisation within the skin. In the dermis, macrophages $(\mathrm{M} \varphi)$, expressing cell-surface marker CD163 can also act as APC. In the steady state, dermal resident macrophages are rare in the porcine skin ${ }^{7}$, but after injury, inflammation or vaccination circulating monocytes recruited from blood differentiate into mature macrophages in the skin ${ }^{15,17}$.

Inactivated porcine reproductive and respiratory syndrome virus (iPRRSV) was selected as vaccine-antigen to explore early immune response following skin vaccination. PRRSV is an important swine pathogen causing major worldwide economic losses ${ }^{18-20}$. As such, there is a need for effective PRRSV vaccines ${ }^{21,22}$, which can already be administered to newborn or neonatal piglets ${ }^{23}$. Neonatal immune responses are directed towards a Th2 response, limiting both Th1 and B-cell responses in neonates compared to adults, often resulting in a reduced vaccine efficacy ${ }^{24-26}$. Moreover, murine studies have shown that neonatal LCs ${ }^{27-29}$ were less effective APCs than adult LCs, which could influence skin vaccination in neonatal pigs. Therefore, it seems appropriate to apply a strategy that intensifies immune responses in the skin by stimulating APCs. It has been reported that potent immuno-stimulators, such as TLR agonist (TLRa) enhanced neonatal immune responses ${ }^{30,31}$. Therefore these could contribute to more effective vaccine responses in neonates after skin vaccination. In this study three different TLRa, i.e.TLR1/2a (Pam3Cys), TLR7/8a (R848) and TLR9a (CpG ODN) were selected as they have shown potential to contribute to APC activation in blood of neonatal and adult pigs ${ }^{32-34}$. TLR7/8a and TLR9a have already proven efficacy after skin vaccination in adult pigs ${ }^{35,36}$ and mice ${ }^{37}$, but to our 
knowledge no studies have evaluated the efficacy of skin vaccination in neonatal pigs with TLRa.

In the present study, neonatal and adult pigs were administered vaccines to the skin using DMN-patches containing iPRRSV-antigen with different TLRa as adjuvant. We investigated the potential of different TLRa to induce early immune responses in the skin and draining $\mathrm{LN}$ and whether these early immune responses were age-dependent.

\section{Materials and methods}

\section{Vaccines}

The vaccine-antigen consisted of binary ethylenimine (BEI) inactivated PRRSV type 1 strain $07 V 063$ (iPRRSV, each dose is $1.0 \times 10^{8} \mathrm{TCID}_{50}$ ). This antigen was prepared as described previously ${ }^{38}$. Three different vaccines were prepared for the vaccination of neonatal and adult pigs each containing a different TLRa as adjuvant: TLR1/2a, Pam3Cys L2000 from EMC Micro-collections; TLR7/8a, R848, Resiquimod from InvivoGen; and TLR9a, CpG ODNtype A sequence D32, ggTGCGTCGACGCAGggggg, from Eurofins Genomics. A full vaccine dose contained $250 \mu \mathrm{g}$ of the individual TLRa mixed with iPRRSV.

Additionally, three different DMN-patches were produced for use in neonates only, that contained either a) the vaccine-antigen (iPRRSV) only or b) iPRRSV with an oilin water (O/W) stable squalene (SE) emulsion ${ }^{39}(29 \%$ volume per volume $(\mathrm{v} / \mathrm{v}))$ and a mixture of the three TLRa (SE+TLRa) or c) squalene emulsion without the TLRa mixture (SE). This TLRa combination mixture contained $80 \mu \mathrm{g}$ of each of the three TLR agonists mentioned above. The SE and SE+TLRa patches were only evaluated for macroscopic and histopathologic changes. The DMN-patches were prepared as previously described ${ }^{38,40}$, using trehalose and polyvinylalcohol (PVA) as excipients. The iPRRSV and TLRa were dispersed homogenously throughout the full volume of the microneedle pore. One full vaccine dose was contained in 200 microneedles, which were $500 \mu \mathrm{m}$ in height. Placebo patches, containing excipients only, were also produced and administered.

\section{Animals and experimental design}

Six $(n=6)$ male twelve-week-old pigs and twelve $(n=12)$ male four-day-old piglets (Topigs Norsvin Z-line, commercial breed) from 4 different sows with the same parity were used. The pigs were purchased from a PRRSV-negative, defined high health status farm (van Beek SPF Varkens B.V., the Netherlands). The adult experiment started after one week of acclimatization, the neonatal experiment started after two days of acclimatization (neonatal pigs were weaned at 1-2 days of age). The animal experiment was conducted in accordance with the Dutch animal experimental and ethical requirements and the project was approved by the Dutch Central Authority for Scientific Procedures on Animals (CCD) (Permit number: ADV401002015356).

All of the pigs, adult and neonate, received one vaccine-dose with one specific TLRa on the medial side of each hind-leg ( $n=4$ for every TLRa, Table 1). In adults, on the skin of 
the the left leg two patches (200 DMN in total) containing iPRRSV and either TLR1/2a or TLR9a $(250 \mu \mathrm{g})$ and one placebo patch (Table 1) were administered. On the right leg of adult pigs 2 patches (200 DMN in total) containing iPRRSV and either TLR9a or TLR7/8a $(250 \mu \mathrm{g})$ and one placebo patch (Table 1 and supplementary Fig1A) were administered. Due to the smaller size of neonates and more limited skin surface we used a different shape of patches in neonates, however the total vaccine dose was the same as in the adult pigs. However, in neonates the placebo patch i.e. without vaccine, was administered to separate animals. In neonates, the left leg was administered with four patches (200 DMN in total) containing a full dose of iPRRSV or iPRRSV with TLR1/2a or TLR9a ( $250 \mu \mathrm{g})$. Neonatal right legs received 4 patches containing iPRRSV with TLR7/8a, $(250 \mu \mathrm{g})$ or SE or SE+TLRa or they received placebo patches (Table 1 and supplementary Fig 1B).

To macroscopically evaluate the local reaction in the skin, six hours post-administration, half of the vaccine-patches were removed from each leg (one vaccine patch from adults or 2 vaccine patches from neonates) and one placebo patch was removed from each adult pig. The remaining patches were removed at 24 hours, which was the time of necropsy. During necropsy skin biopsies ( $8 \mathrm{~mm}$ punch biopsies) were taken from treated and untreated skin (non-vaccinated; NV). The skin of the caudal ventral abdomen on the right side (comparable skin thickness as medial side hind-leg) was selected as NV skin. The right axillary lymph node was sampled as control lymph node next to the draining lymph node (superficial inguinal lymph node).

Table 1: Skin vaccines used in adults and neonatal pigs.

\begin{tabular}{|c|c|c|c|c|}
\hline Animal & \multicolumn{2}{|c|}{ Left leg } & \multicolumn{2}{|c|}{ Right leg } \\
\hline \multicolumn{5}{|l|}{ Adult } \\
\hline$\# 1,2$ & TLR1/2a & placebo & TLR9a & placebo \\
\hline$\# 3,4$ & TLR1/2a & placebo & TLR7/8a & placebo \\
\hline$\# 5,6$ & TLR9a & placebo & TLR7/8a & placebo \\
\hline \multicolumn{5}{|c|}{ Neonate } \\
\hline$\# 1$ to 4 & \multicolumn{2}{|c|}{ TLR1/2a } & \multicolumn{2}{|c|}{ TLR7/8a } \\
\hline$\# 5$ to 8 & \multicolumn{2}{|c|}{ Antigen } & \multicolumn{2}{|c|}{ Placebo } \\
\hline$\# 9,10$ & \multicolumn{2}{|c|}{ TLR9a } & \multicolumn{2}{|c|}{ SE } \\
\hline$\# 11,12$ & \multicolumn{2}{|c|}{ TLR9a } & \multicolumn{2}{|c|}{$\mathrm{SE}+\mathrm{TLRa}$} \\
\hline
\end{tabular}

The dissolving-microneedle (DMN) patches contained different Toll-like receptor agonists (TLRa) as adjuvant: TLR1/2a (Pam3Cys), TLR7a (R848) and TLR9a (CpG ODN) combined with inactivated porcine reproductive and respiratory syndrome virus (iPRRSV). Each pig received two different DMN-patches, one on the left leg and on one on the right leg. Placebo patches without vaccine were applied on each leg of the adult pig next to the vaccine. Only the neonates received patches with only iPRRSV (antigen) and additionally patches with a stable emulsion (SE) with a mixture from the three TLRa (SE+TLRa) or without (SE). 


\section{Assessment of post-vaccination reaction: macroscopy and histology}

After removal of the DMN-patches, at $6 \mathrm{~h}$ and $24 \mathrm{~h}$ after patch application, the skin was graded from 0 (no visible change) to 3 (discolouration and/or swelling of the skin) ${ }^{38}$. Skin biopsies taken $24 \mathrm{~h}$ after patch application were fixed in $10 \%$ neutral buffered formalin and routinely processed into paraffin-embedded tissue samples. Three consecutive $4 \mu \mathrm{m}$ thick sections were cut and stained with haematoxylin and eosin (HE). HE stained slides were semi-quantitatively analysed in a "blinded" manner per animal by a board-certified veterinary pathologist. Each section was graded for number of inflammatory cells, hyperaemia and dermal oedema, resulting in an overall score from 0-3. Detailed scoring of histology is described in supplementary data (Table S1A). Additionally, the NV neonatal and adult skin was compared by measuring the epidermal and dermal thickness (three measurements per animal, objective $2 \mathrm{x}$ ) with cellSense software $\mathrm{V} 1.16$ from Olympus.

\section{Identification of antigen presenting cells in the skin}

For cell phenotyping skin biopsies were snap-frozen on dry-ice in Tissue-Tek ${ }^{\oplus}$ optimal cutting temperature compound (O.C.T. $\left.{ }^{\mathrm{TM}}\right)$. Cryosections $(8 \mu \mathrm{m})$ were cut with a Leica CM3050S cryostat. Before processing for immunohistochemistry (IHC) with 3,3'-diaminobenzidine (DAB) or immunofluorescence (IF) microscopy, tissue sections were air-dried, fixed in cold acetone and stained with mouse anti-pig CD1a (IgG2a, clone 76-7-4 from Southern Biotech). For IHC the anti-CD1a was incubated for $4 \mathrm{~h}$ at room temperature. The ChemMate Envision Detection $\mathrm{kit}^{\mathrm{TM}}$ /mouse with DAB from Dako was used according to manufactures' instruction for antigen visualisation. IHC slides were analysed semiquantitatively in a "blinded" manner and graded based on the number of positive staining cells in epidermis (0-3 for LC) and dermis (0-3 for dDC). Detailed IHC-scoring is described in the supplementary data (Table S1B).

For IF microscopy, skin samples were stained using the three primary antibodies: CD1a (as earlier described), mouse-anti-pig MHCII (IgG1, clone K247.3G8 from Bio-rad) and mouse anti-pig CD163 (IgG1, clone 2A10/11 from Bio-rad). The following secondary antibodies were used: Alexa Fluor $647^{\circ}$ goat anti-mouse (lgG2a fro6m Bio-rad) and Alexa Fluor $568^{\circ}$ goat anti-mouse (IgG1a from Bio-rad) (supplementary data Table S2). Hoechst 33342 staining dye solution (Sigma-Aldrich) was applied for nuclear staining. From every skin sample 3 different images were acquired using consistent microscope settings (Leica DM6b upright microscope, 20x objective) and positive cells were selected manually and counted with the Leica Application Suite X software (version 2.0.0.14332) . For negative controls (IHC and IF microscopy), the primary antibodies were replaced by isotype controls or only the secondary antibodies were used. 


\section{Gene analysis with high throughput qPCR}

\section{Gene selection}

Based on literature search and previous porcine expression studies of subcutaneous tissue ${ }^{41}$, we selected 86 genes, likely involved in the early immune response after skin vaccination. For 10 important genes two different primer sets were selected. The genes were divided into 5 main clusters, which are presented in the results section. Primer sequences, amplicon lengths and if applicable literature reference for selected genes are described in the supplementary data. (Table S3).

\section{RNA extraction and CDNA synthesis}

Skin biopsies and lymph nodes were snap-frozen on dry-ice and stored at $-80^{\circ} \mathrm{C}$. Before RNA extraction samples were homogenized in TRIZOL ${ }^{\circ}$ and RNA was extracted by using the Directzol ${ }^{\circ}$ RNA MiniPrep (Zymo Research) according to the manufacturer's instructions. RNA quantity and purity were assessed with the NanoDrop $1000^{\mathrm{Tm}}$ (Thermo Fisher Scientific). The purity was evaluated based on optical density (OD) using the A260/280 and A260/230 ratios. RNA-quality was assessed by measuring the RNA integrity number (RIN) on an Agilent Bioanalyzer (Agilent Technologies) using the RNA 6000 nano kit (Agilent Technologies). Samples with a RIN $>5$ were considered appropriate for further processing. The lymph node samples had a mean RNA integrity of $7.9 \pm 0.8$ (SD), while the skin samples had a lower average RIN value of $6.6 \pm 0.8$ (SD) (results not shown). From each RNA sample, duplicate CDNA syntheses were made from $500 \mathrm{ng}$ extracted RNA using the QuantiTect Reverse Transcription kit from Qiagen according to manufacturer's instructions. The CDNA was diluted 1:10 in low-ethylenediaminetetraacetic acid (EDTA) TE-buffer (Panreac AppliChem) before pre-amplification. $3 \mu \mathrm{l}$ TaqMan PreAmp Master Mix (Applied Biosystems), $2.5 \mu \mathrm{l} 200 \mathrm{mM}$ mix of all primers used subsequently for qPCR, $24 \mu$ low- EDTA TE-buffer (Panreac Applichem) and $2.5 \mu$ l diluted CDNA was mixed and incubated at $95^{\circ} \mathrm{C}$ for 10 minutes followed by 20 cycles of $95^{\circ} \mathrm{C}$ for 10 seconds and $60^{\circ} \mathrm{C}$ for four minutes. The pre-amplified cDNA was treated with $16 \mathrm{U}$ Exonuclease I (New England Biolabs) for 30 minutes at $37^{\circ} \mathrm{C}$ and stored at $-20^{\circ} \mathrm{C}$ until further processing.

\section{High-throughput $q P C R$}

qPCR was performed using 96.96 Dynamic array integrated fluid circuit (IFC) chips (Fluidigm) in the BioMark HD System (Fluidigm), combining 96 primer sets with 96 samples. Each primer mix contained $3 \mu \mathrm{l} 2 \mathrm{X}$ Assay Loading Reagent (Fluidigm) and $3 \mu \mathrm{l}$ of $20 \mathrm{mM}$ forward and reverse primers suspended in low-EDTA TE-buffer (Panreac AppliChem). The sample mixes were prepared using $3 \mu \mathrm{l} 2 \mathrm{X}$ TaqMan Gene Expression Master Mix (Applied Biosystems), $0.3 \mu \mathrm{l}$ 20X DNA Binding Dye Sample Loading reagent (Fluidigm), $0.3 \mu \mathrm{l} 20 \mathrm{X}$ Evagreen (Panreac AppliChem), $0.9 \mu$ l low-EDTA TE-buffer (Panreac AppliChem) and 1.5 $\mu \mathrm{l}$ pre-amplified diluted (1:10) cDNA. Before loading samples and primers, the chip was primed in an HX IFC controller (Fluidigm). After priming, $5 \mu \mathrm{l}$ of each sample mix and 
primer mix were distributed into the appropriate compartments and loaded into the chip in the HX IFC controller. Thereafter the chip was inserted in the BioMark real-time PCR instrument (Fluidigm) and the following program was used: two minutes at $50^{\circ} \mathrm{C}$ and 10 minutes at $95^{\circ} \mathrm{C}$, next 35 cycles with 15 seconds at $95^{\circ} \mathrm{C}$ and one minute at $60^{\circ} \mathrm{C}$. Non-template controls and non-reverse transcriptase controls were included to indicate problems with contamination, non-specific amplification or genomic DNA, respectively. Standard curves constructed from three separate dilution series of pooled cDNA of all samples to determine the efficiency of each primer. Based on the melting curves, standard curves and control samples 13 genes (HPRT1 (25), CCL2 (117), CCR7 (608), GZMA (758), IL8 (37), SAA (158), TNF (125), IL12A (44), IL13 (279), IL23A (195), TLR7 (164)

TLR8 (127), TLR9(81) were excluded from the skin sample analysis and 7 genes (HPRT1 (25), CCL2 (117), GZMA (758), IL13 (279), IL23A (195), IFNL3(298), IFNB (223) from the lymph node sample analysis.

\section{4. $q P C R$ data analysis}

Raw data were inspected using Fluidigm Real-Time PCR Analysis software (v. 4.1.3). GeneEx5

(v. 5.4.4.119) (MultiD) was used for data pre-processing. To compensate for variation between dynamic chips, three pooled samples were used as interplate calibrators. Out of six tested reference genes, actin beta (ACTB), glyceraldehyde-3-phosphate dehydrogenase (GAPDH), peptidylprolyl isomerase A (PPIA) and tyrosine 3-monooxygenase/tryptophan 5-monooxygenase activation protein zeta (YWHAZ), were found to have the most stable expression in porcine skin and lymph nodes using both GeNorm and NormFinder in (GeneEx5 software). Expression data were normalized following the approach of Pfaffl ${ }^{42}$ with geometric averaging of relative quantities of reference genes ${ }^{43}$. Gene expression changes were normalized against the control sample of the same animal. For this purpose, duplicated samples of the control were averaged. Relative expression of genes (foldchange) of test samples was calculated for individual replicate separately and passed to the following statistical analysis. Normalization was performed with 'in-house' script.

\section{Statistical analysis}

The data of the macroscopic changes, histology and IHC were analysed with a nonparametric Kruskal-Wallis test followed by a post-hoc Dunnett test for multiple comparisons. The data of the IF microscopy were analysed with a one-way ANOVA followed by a post-hoc Dunnett test for multiple comparisons. Statistical assessment of differences between NV samples for adults and neonates was performed with an unpaired t test (twotailed with a Welch's correction). Statistical analysis was performed with GraphPad Prism 8.2.1 software. $P$ - values less than 0.05 were considered significant statistically $\left({ }^{*} p<0.05\right.$, $\left.{ }^{* *} p<0.01,{ }^{* * *} p<0.001\right)$. 
Statistical assessment of expression changes of selected genes during the experiments was performed using one-way ANOVA test (one parameter, treatment) or two-way ANOVA (two parameters, treatment and age, and their interaction). Influence of individual treatments on gene expression was analysed with Mann-Whitney $U$ test. Holm-Bonferroni correction was used to adjust $\mathrm{p}$-value threshold for multiple testing. Statistical analysis was performed using Python 2.7.15 and modules sklearn, statsmodels (biopython version 1.70), pandas (version 0.23.4), numpy (version1.15.1) and matplotlib (version 2.2.3).

Table 2: Comparison non-vaccinated adult and neonatal skin.

\begin{tabular}{|c|c|c|}
\hline \multicolumn{3}{|c|}{ Histology normal skin (HE) } \\
\hline Thickness & adult & neonate \\
\hline Epidermis & $62 \mu \mathrm{m} \pm 11$ & $41 \mu \mathrm{m} \pm 5^{*}$ \\
\hline Dermis & $1263 \mu \mathrm{m} \pm 107$ & $698 \mu \mathrm{m} \pm 160^{* * *}$ \\
\hline \multirow[t]{3}{*}{$\# 5,6$} & TLR9a & placebo \\
\hline & \multicolumn{2}{|c|}{ Identification APCs skin } \\
\hline & IFT & $\mathrm{IHC}$ \\
\hline \multicolumn{3}{|l|}{ Epidermis } \\
\hline LC & $\mathrm{CD} \mathrm{a}^{+} \mathrm{MHClI}^{+} \mathrm{CD} 163^{-}$ & $\mathrm{CD}_{1 \mathrm{a}^{+}}$ \\
\hline \multicolumn{3}{|l|}{ Dermis } \\
\hline dDC & $\mathrm{CD}_{12}{ }^{+} \mathrm{MHClI}^{+} \mathrm{CD} 163^{+/-}$ & $\mathrm{CD}_{1} \mathrm{a}^{+}$ \\
\hline $\mathbf{M \phi}$ & $\mathrm{CD}_{1} \mathrm{a}^{-1+} \mathrm{CD}_{163^{+}}$ & Not evaluated \\
\hline
\end{tabular}

Adult $(n=6)$ and neonatal skin $(n=12)$ were compared for epidermal and dermal thickness with a haematoxylin and eosin (HE) stain. The neonatal dermis and epidermis were significantly thinner than the adult dermis and epidermis. The different antigen presenting cells (APC): Langerhans cell (LC), dermal dendritic cell (dDC) and dermal macrophages $(M \phi)$ were evaluated with immunohistochemistry (IHC) and immunofluorescence (IF) microscopy using different surface markers.

\section{Results}

\section{In-situ comparison of normal non-vaccinated adult and neonatal skin}

The skin histology and presence of different types of APCs in the skin of NV (control) adult and neonatal pigs were evaluated and compared to identify possible age-dependent differences, which could influence the effect of skin vaccination (Table 2). 

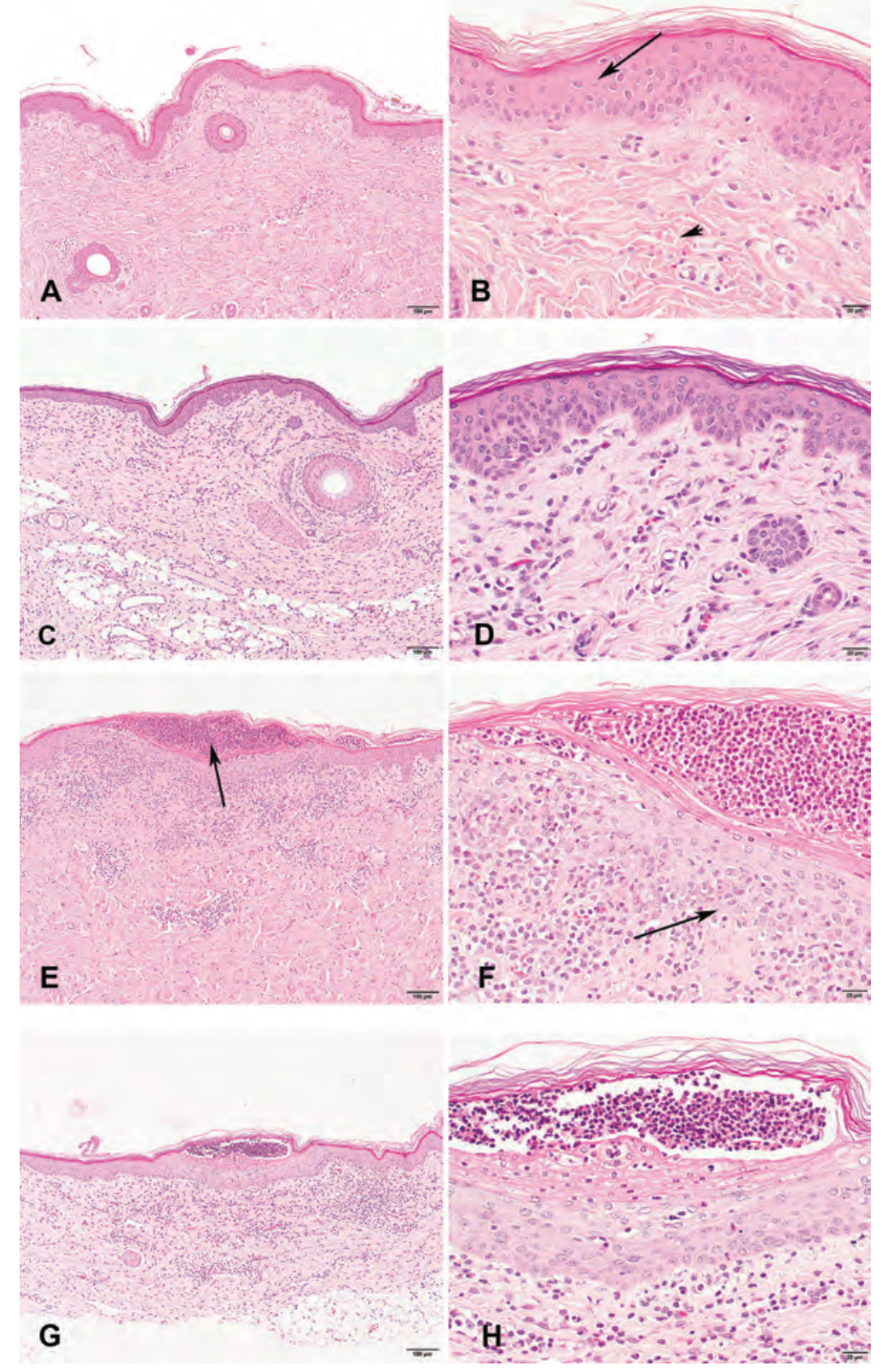

Figure 1: Skin histology in adult and neonatal pigs.

Skin biopsies were stained with haematoxylin and eosin (HE). Non-vaccinated (NV) adult control skin (A, B) with epidermis (arrow) and dermis arrowhead). The NV neonatal control skin (C, D) is more cellular in dermis; Skin vaccination with Toll-like receptor $7 / 8$ agonist (TLR7/8a) induced grade 3 changes in adult $(E, F)$ and neonatal $(G, H)$ skin characterized by a focal extensive infiltration of mononuclear inflammatory cells and lesser numbers of neutrophils in the superficial dermis extending to the epidermis with pustule formation ( $E$, arrow). Occasionally, the dermal-epidermal junction was affected ( $F$, arrow) and the dermis showed multifocal hyperaemia and oedema. 
The neonatal and adult skin had a similar structure, however the epidermis and especially the dermis were significantly thicker (1.5-fold and 1.8-fold, respectively) in the adult skin compared to the neonatal skin. For both adult and neonates, the skin thickness was larger $(>500 \mu \mathrm{m})$ than the length of the DMN. The adult and neonatal dermis contained a comparable variety of adnexal structures (i.e. hair follicle, sweat glands and sebaceous glands), however the neonatal dermis was distinctly more cellular in the HE stain (Fig.1 A and C), most likely due to a larger number of fibroblasts and immune cells.
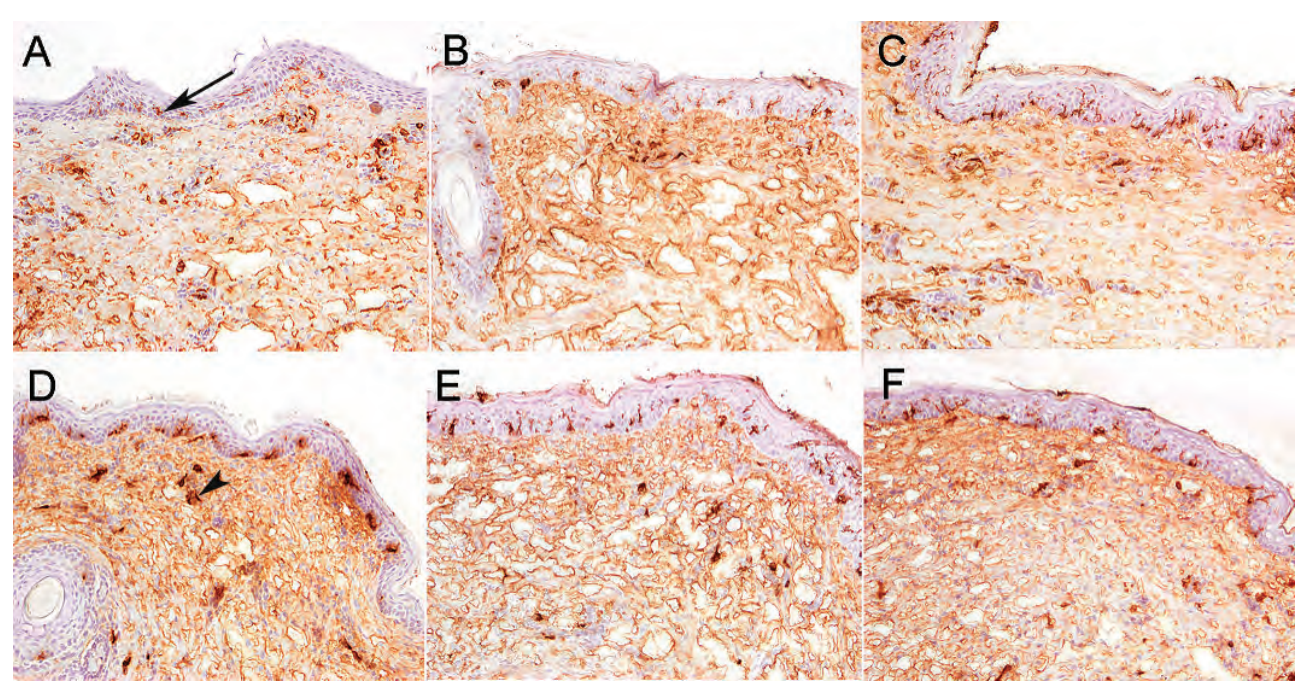

Figure 2: $C D 1$ a expression in adult and neonatal skin.

Adult ( $A, B$ and $C$ ) and neonatal (D, E and F) skin was evaluated for CD1a expression with immunohistochemistry (IHC) (objective 20x) to identify Langerhans cells (LC) in the epidermis (arrow (A)) and dermal dendritic cells (dDCs) in the dermis (arrowhead (D)). The CD1a expression is comparable in NV adult and neonatal skin ( $A$ and $D)$ and skin treatment with placebo vaccine ( $B$ and E) and or TLR7/8a vaccine (C and F) does affect the CD1a expression in epidermis or dermis. 


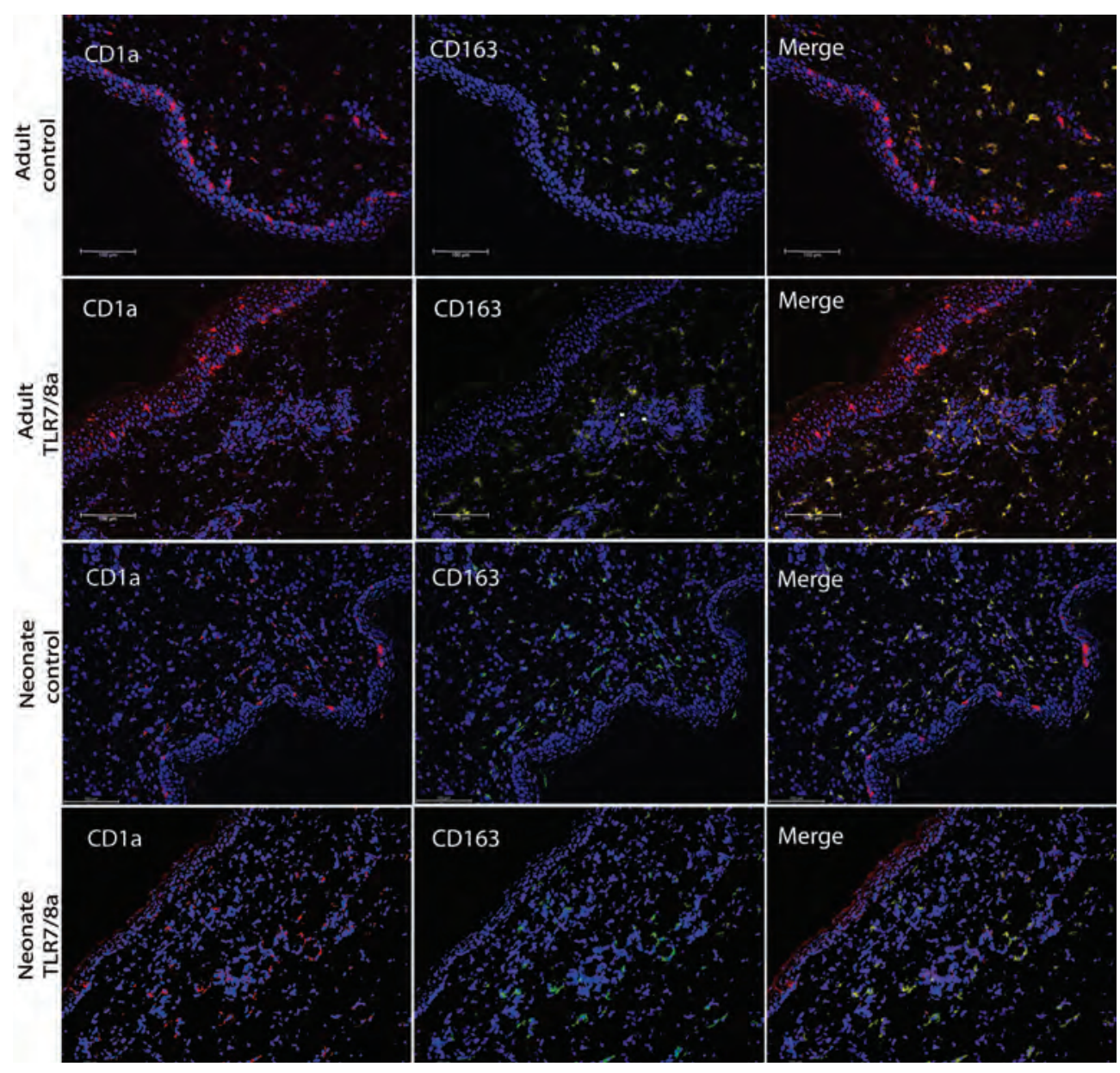

Figure 3: Antigen presenting cells (APCs) in adult and neonatal skin.

In non-vaccinated (NV) control skin and in Toll-like receptor (TLR) 7/8 agonist vaccinated skin of adult and neonatal pigs different APCs were in-situ localized with surface-markers CD1a (red) to identify dendritic cells and CD163 (green/yellow) to identify macrophages. Objective used 20x, scale bars $=100 \mu \mathrm{m}$.

To characterize and quantify the number of APCs within the epidermis and dermis, we used surface-marker CD1a in IHC (Fig. 2) and surface-markers CD1a, MHCII and CD163 in IF microscopy (Fig. 3). Using IF microscopy all CD1 ${ }^{+}$cells in the epidermis (LC) were $\mathrm{MHClI}^{+}$and $\mathrm{CD} 163^{-}$and localized near the basement membrane (Fig. 3). The number of LCs was comparable in the NV epidermis of adult and neonatal pigs (Fig. 4A, C and D). In the dermis, the $\mathrm{dDC}$ s were $\mathrm{CD} 1 \mathrm{a}^{+}$in $\mathrm{IHC}$ and $\mathrm{CD} 1 \mathrm{a}^{+} \mathrm{MHCll}^{+}$or $\mathrm{CD} 1 \mathrm{a}^{+} \mathrm{CD} 163^{+/}$in IF microscopy. All CD1 $\mathrm{a}^{+}$cells in epidermis and dermis also expressed $\mathrm{MHCll}$, in both neonates and adults (results not shown). The dDCs were mainly located in the superficial dermis near 
the epidermal basement membrane in the proximity of small blood vessels. There was no significant difference in the number of $\mathrm{dDC}$ in neonatal and adult pigs based on the results of the IHC and IF microscopy (Fig. 4B and E). The CD1 $\mathrm{a}^{+/-} \mathrm{CD} 163^{+}$macrophages (M $\left.\phi\right)$ were located in the superficial and deeper dermis and the number of macrophages was significantly higher in the dermis of NV neonatal pigs compared to NV adult pigs (Fig. 4F).

A

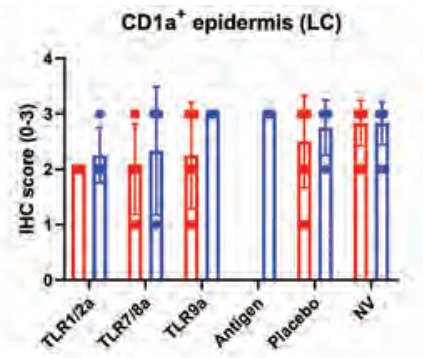

C

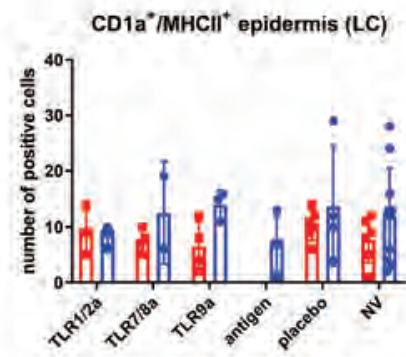

$\mathrm{E}$

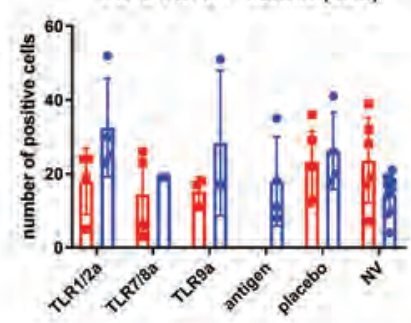

B

- adult

- neonate
CD1 $\mathrm{a}^{+}$dermis (dDC)

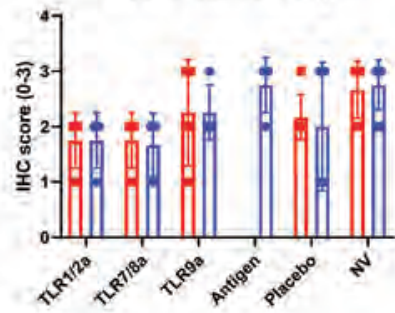

D

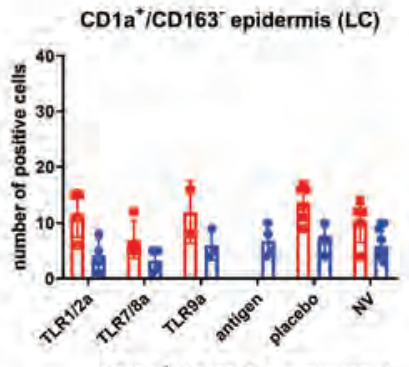

$\mathbf{F}$

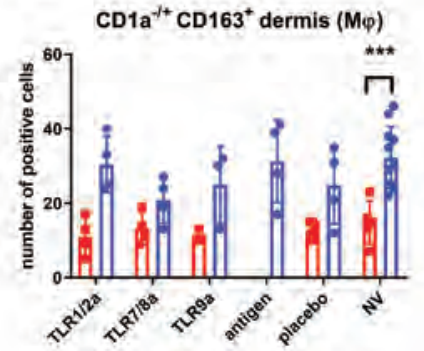

Figure 4: Quantification of antigen presenting cells (APCs) in adult and neonatal skin after dissolving microneedle (DMN)-patch vaccination.

The number of APCs was graded in the adult and neonatal skin with immunohistochemistry (0-3) (A and B) or counted with immunofluorescence microscopy (IF) microscopy (C,D, E and F), three different fields with $20 x$ objective. After vaccination (24h) with different Toll-like receptor (TLR) agonists, antigen only or with placebo patches, the skin was evaluated for Langerhans cells (LCs) in the epidermis ( $A, C$ and $D)$, dermal DCs $(d D c)$ in the dermis $(B$ and $E)$ and macrophages $(M \phi)(F)$ in the dermis. Each symbol represents one animal (adult $=$ red square and neonate $=$ blue circle). The line indicates the median with S.D. (A and B) or the mean with the $95 \% \mathrm{Cl}(\mathrm{C}, \mathrm{D}, \mathrm{E}$ and F). Statistical significance was calculated for the different vaccines compared to non-vaccinated (NV) animals for every age-group or for NV neonatal samples compared to NV adult samples. 
A
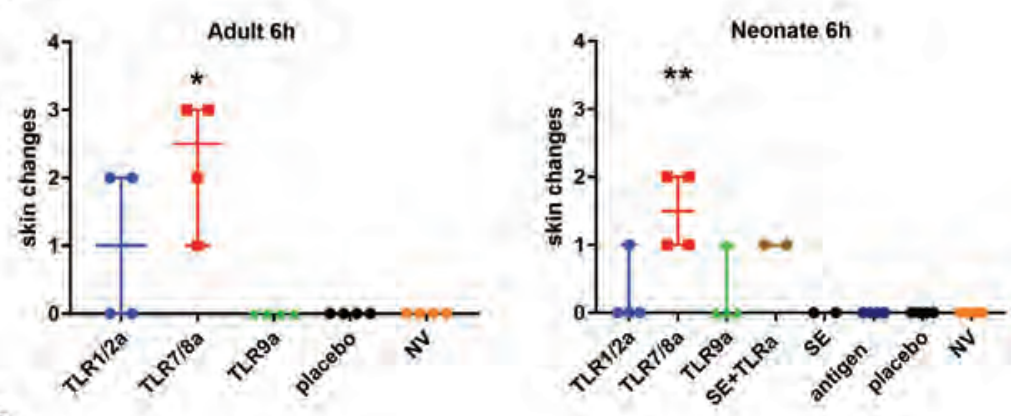

B
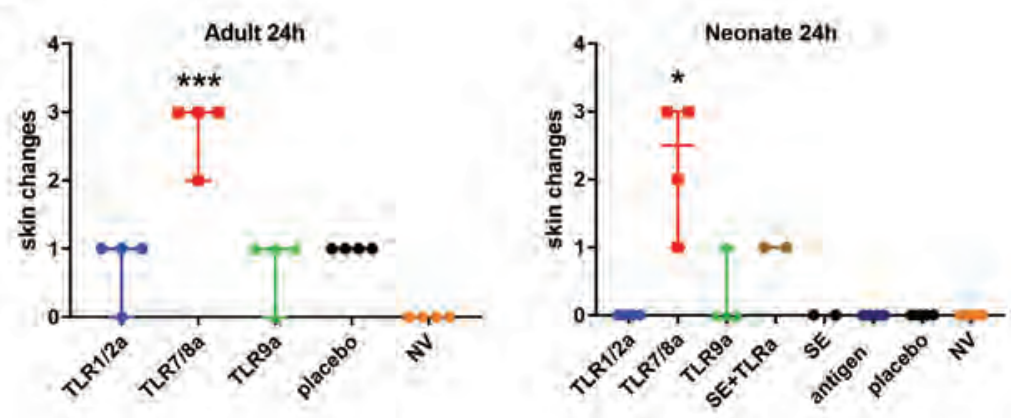

Figure 5: Macroscopic skin changes after skin vaccination using dissolving-microneedle (DMN) patches.

Skin reaction overview for adult and neonatal pigs $6 \mathrm{~h}(\mathrm{~A})$ and $24 \mathrm{~h}(\mathrm{~B})$ after skin vaccination with vaccines containing different Toll-like receptor (TLR) agonists, a squalene emulsion (SE), a mixture of TLR agonists and SE (SE+TLRa), antigen only or a placebo patch without vaccine. Each symbol represents one animal, line indicates the median of a group $(n=4)$ with the $95 \% \mathrm{Cl}$. ${ }^{*} p<0.05,{ }^{* *} p<$ $0.01,{ }^{* * *} p<0.001$ ) compared to non-vaccinated (NV) control skin).

\section{Macroscopic and histopathological changes after skin vaccination}

Six hours after application of the TLR7/8a patch, a macroscopic grade 1 to 3 skin reaction was induced in all adult and neonatal pigs, which continued with a similar intensity to 24 hours after vaccination (Fig. 5). In both age-groups the TLR1/2a and 9a patches induced only a mild and occasionally moderate skin reaction (grade 0 to 2), which was significantly less severe than the TLR7/8a patch and comparable to the placebo patch.

These findings were supported by the histopathologic changes (Fig.6), where both neonates and adults the TLR7/8a patch induced mainly grade 3 changes characterized by a focal extensive infiltration of mononuclear inflammatory cells and lesser numbers of neutrophils in the superficial dermis extending to the epidermis with pustule formation. Occasionally, the dermal-epidermal junction was affected and the dermis showed multifocal hyperaemia and oedema (Fig.1). The histopathologic changes induced by 
the TLR1/2a and 9a patches were restricted to maximum grade 2 in both neonatal and adult pigs, where adult pigs showed more skin changes for TLR1/2a (macroscopy and histology) than neonatal pigs. The placebo-patch induced macroscopically a mild brown discoloration of the skin (data not shown), which was not associated with histopathologic changes in the adult pigs only.

A

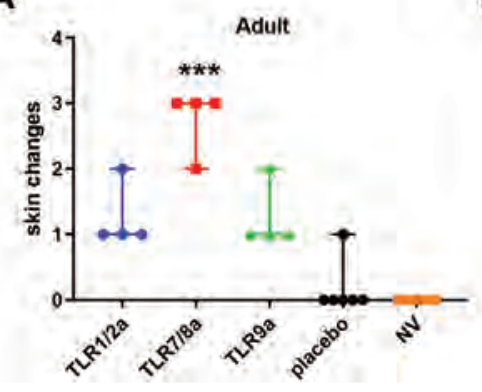

B

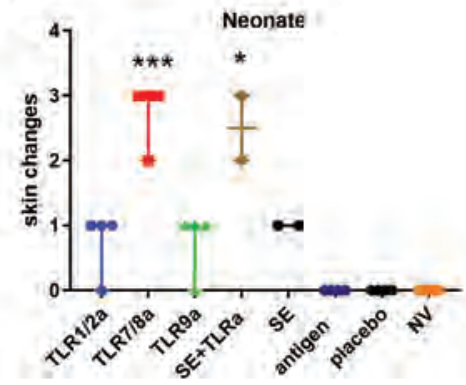

Figure 6: Skin histology after vaccination using dissolving-microneedle (DMN) patches.

The patches contained vaccines with different Toll-like receptor (TLR) agonists, a squalene emulsion (SE), a mixture of TLR agonists and SE (SE+TLRa), antigen only or a placebo patch without vaccine. Skin biopsies taken $24 \mathrm{~h}$ after vaccination were graded from 0-3 for adult (A) and neonatal (B) pigs. Each symbol represents one animal, line indicates the median of a group $(n=4)$ with the $95 \% \mathrm{Cl}$. $\left({ }^{*} \mathrm{p}\right.$ $\left.<0.05,{ }^{* *} \mathrm{p}<0.01\right)$ compared to non-vaccinated (NV) control skin.

\section{In-situ characterization of antigen presenting cells after skin vaccination}

Both IHC and IF microscopy were used to quantify the number of DCs (LC and IDC) and dermal macrophages in the skin $24 \mathrm{~h}$ after vaccine administration. The number of $\mathrm{LC}$ in the epidermis (CD1 $\left.{ }^{+}\right)$in IHC and CD1 ${ }^{+} / \mathrm{MHCll}^{+}$or CD1 $\mathrm{a}^{+} \mathrm{CD} 163^{-}$in IF microscopy (Fig. 4) did not significantly change compared to the NV skin in both the adult and neonatal pigs. However, after skin vaccination the dendrites of the LC were more clearly visible in the epidermis compared to the NV control skin with a localisation of the cell bodies directed toward the outer surface of the epidermis. This morphological change of the LCs was observed in both age-groups (Fig.2 and 3).

The number of $d D C s$ did not significantly change after vaccine administration to the skin compared to the NV skin in the adult and neonatal pigs as observed with $\mathrm{IHC}$ for expression of $\mathrm{CD} 1 \mathrm{a}$ and with IF microscopy for dermal expression of $\mathrm{CD} 1 \mathrm{a}^{+} \mathrm{MHCll}^{+}$. Also, the number of dermal $M \phi\left(C D 163^{+}\right)$did not change significantly following vaccination (Fig. 4). For both IHC and IF microscopy there was a large variation within the different samples of neonate pigs and within the different samples of adult pigs. 


\section{Gene expression in skin and lymph node}

The selected immune genes were grouped according to gene ontogeny (GO) in 6 different clusters (Table S3): (1) APC activation and migration, (2) acute phase inflammatory response; (3) anti-inflammatory response, (4) Th directed response, (5) TLR expression and activation, and (6) other/epithelial junction. After data processing and removal of duplicate primers, valid expression data from 74 genes of the skin samples and 74 genes of the LN samples were obtained. Differences in gene expression were considered relevant when a statistically significant fold change of $>2$ or $<0.5$ compared to the reference sample was measured and only the genes with such a fold change are reported below.

1. Gene expression in non-vaccinated adult and neonatal skin and lymph node

To investigate potential age-dependent differences in basal gene expression in NV, control skin and control LN, we compared the level of expression in the neonatal samples with the level of expression in the adult samples for 74 genes (Table S4). The neonatal skin showed a relevant different expression level in 9 of 74 investigated genes. The main difference was the lower expression of APC activation/migration related genes (e.g. CD86 and SLADRB1, surface-markers for antigen presentation) in the neonatal skin. The macrophage surface-marker CD163 was the only gene that was significantly higher expressed in the NV neonatal skin compared to the adult NV skin. We found no difference in TLR gene expression in the neonatal skin compared to the adult skin. Both adult and neonatal skin showed no expression of TLR9, while we did find TLR9 gene expression in the LN of both age-groups.

The neonatal control LN showed a relevant different expression level in 25 of the 72 investigated genes compared to the adult control LN. Genes related to DCs/ antigen presentation (CD1a and CCR7) were less expressed, while genes related to monocytes/ macrophages (CD14 and CD163) were more expressed in the neonatal control LN compared to the adult control LN. All data on biologically relevant genes in neonatal NV skin and control LN compared to adult control samples are provided in supplementary Table S4.

\section{Gene expression after skin vaccination in adult and neonatal pigs}

To investigate the effect of skin vaccination on expression of the selected genes in the vaccinated skin and draining LN, 24 hours after administrations, we calculated the foldchange difference in gene expression between the NV control skin and control LN and the vaccinated skin and vaccinated draining LN. For both age groups, TLR7/8a vaccinated skin showed the most prominent induction of gene expression compared to NV control skin, with the overall number of upregulated genes being higher in neonates (38 of 74 genes) compared to adults (21 of 74 genes). In both age groups, especially the genes in the clusters APC activation/migration (e.g. CCL20, CCR7, CD86 and CXCL10) and TLR expression/activation (e.g. IFNL3, IRF7, OAS1 and RNASEL) were significantly upregulated. 
Neonatal pigs showed a high upregulation (fold-change $>25$ ) of gene expression for the pro-inflammatory genes IL8, GZMB and CASP1 in the skin. No upregulation of these genes was observed in the adult skin samples after TLR7/8a vaccination. In contrast, the overall gene expression in the TLR9a vaccinated skin contained a higher number of downregulated genes in adult and neonatal samples compared to the other TLRa vaccines (Table 4A). This downregulation in gene expression was observed in all immune response clusters, except for the APC cluster (e.g. CCL20, CD86 and CXCL10) in neonatal pigs which showed an upregulation. The TLR $1 / 2$ a vaccine was the only skin vaccine which did not induce a significant change in gene expression in the Th clusters compared to the NV control skin (Table 4A). The placebo vaccination induced mainly a downregulation in gene expression particularly in the adult skin (12 of 74 genes) and to a lesser extend in the neonatal skin (4 of 74 genes), especially for the APC activation/maturation cluster (e.g. CCL3 and CXCR4) and for the acute phase inflammatory response cluster (e.g. DEFB1). The antigen-only vaccine $(\mathrm{Ag})$, only administered in neonates, induced only a regulation in a limited number of genes (3 of 74 genes).

Draining LNs of skin vaccinated with TLR7/8a regulated the expression of a larger number of genes ( 13 of 74 genes neonate and 10 of 74 genes adult) compared to the other skin vaccines in both age groups $(<9$ of 74 genes neonate and $<3$ of 74 genes adult). In both age groups, nearly all upregulated genes in the LN samples of the TLR7/8a vaccine showed a fold-change of $<5$, while a large number of upregulated genes in the TLR7/8a vaccinated skin samples showed a fold-change of $>10$. Thereby the response to vaccination was larger in the skin compared to the draining $L N$. (Table $3 \mathrm{~A}$ and $3 \mathrm{~B}$ ).

Draining LNs of the TLR7/8a vaccine showed particularly a gene upregulation in the pro-inflammatory immune response cluster (TP53, GZMB and SAA) in both age-groups. The upregulation of gene expression in the APC activation and migration cluster (e.g. CCR7, CCL19 and MMP9) was comparable for all the skin vaccines in both age-groups, including the Ag-only vaccine. Overall, the neonatal LNs contained a higher number of regulated genes compared to the adult LNs after skin vaccination, especially for the TLR9a vaccine ( 7 of 74 genes neonate versus 1 of 74 genes adult) and for the TLR1/2a vaccine (10 of 74 genes neonate versus 3 of 74 genes adult). The fold-changes of all significantly regulated genes in the vaccinated skin and draining $L N$ are presented in Table $3 \mathrm{~A}$ and Table 3B, respectively. In Supplementary Fig. S 2 and 3 the $p$-value threshold corrected for multiple testing (MTC) is shown for all analysed genes. 


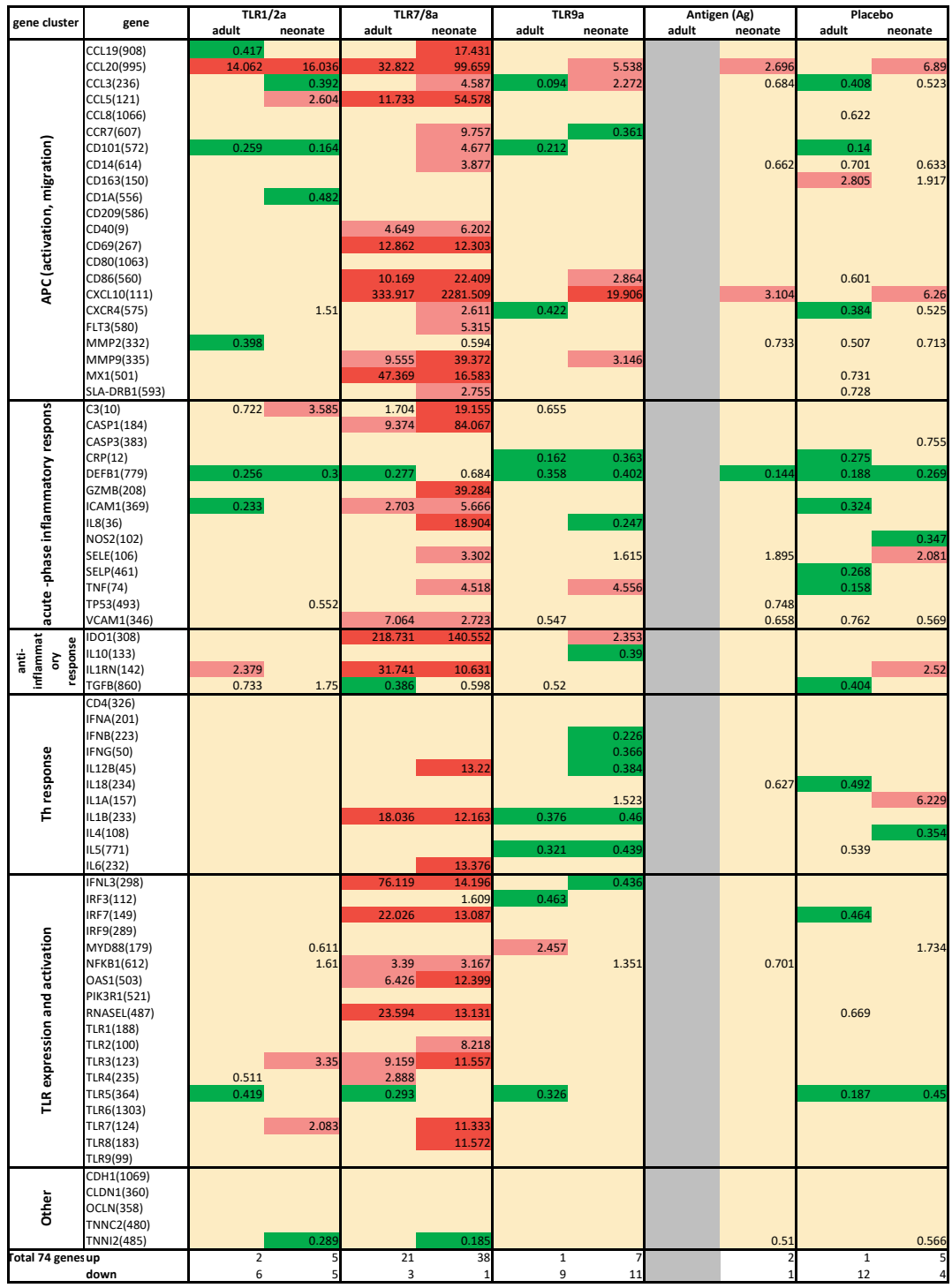

Table 3A: Heat map of relative gene expression in skin after skin vaccination.

For each vaccine only the significant relative fold-changes are presented for immune response genes $24 \mathrm{~h}$ after skin vaccination. The relative fold-change of the individual genes in the vaccinated skin $(n=4)$ compared to the non-vaccinated skin $(n=4)$ is in expressed adult and neonatal pigs. The colour scheme for the relative gene expression is as follows: the yellow results are not significantly changed after vaccination and fold-change is not indicated or have a significant fold-change between 0.5 and $2 ;<0.5$ is dark green (i.e. the respective gene is down regulated); between 2 and 10 is red (i.e. the gene is moderately upregulated) or $>10$ is dark red (i.e. the gene is strongly upregulated). 


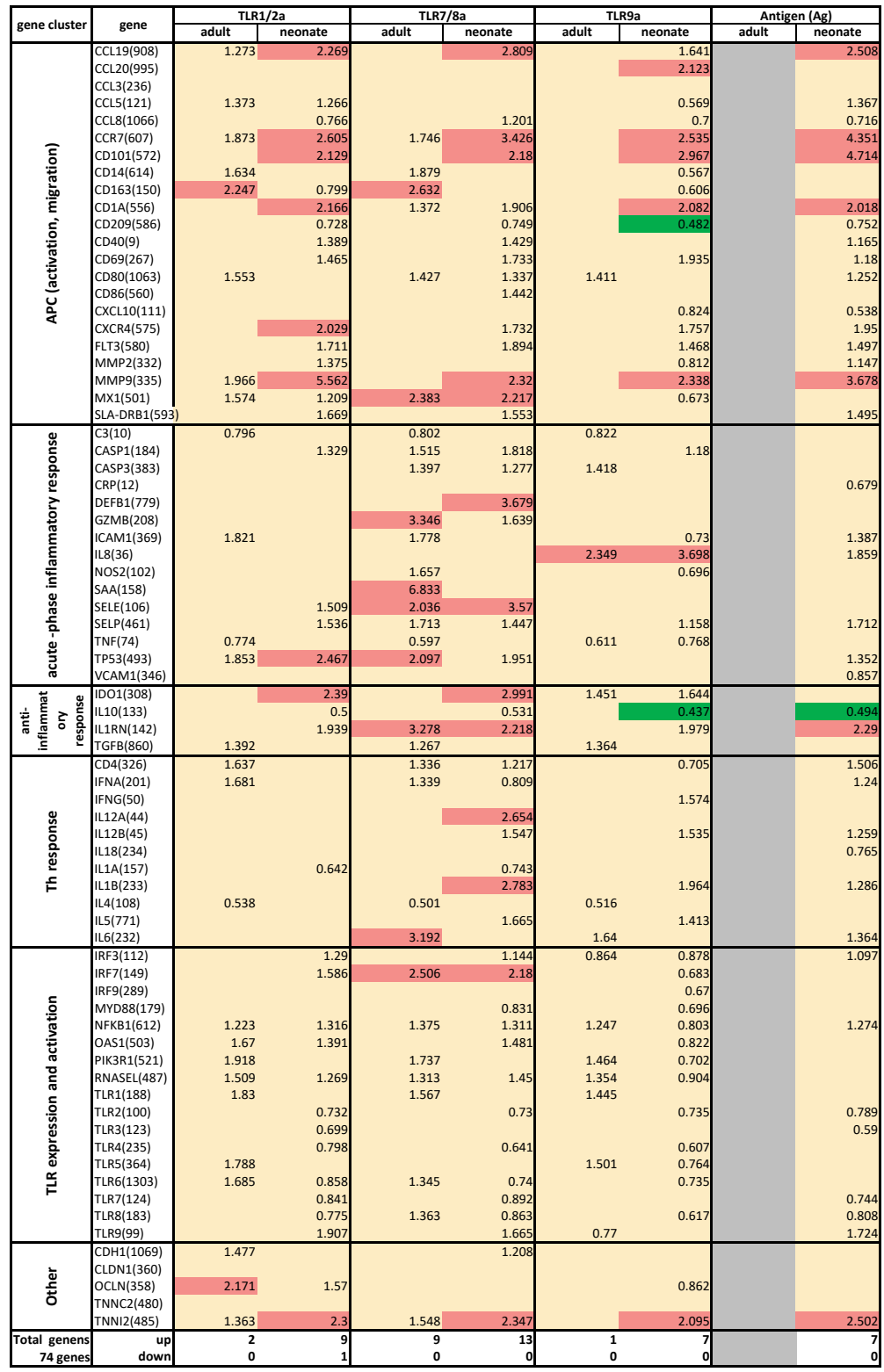

Table 3B: Heat map of relative gene expression in draining lymph node after skin vaccination.

For each vaccine only the significant relative fold-changes are presented for immune response genes $24 \mathrm{~h}$ after skin vaccination. The relative fold-change of the vaccinated draining lymph node $(\mathrm{LN})(n=4)$ compared to the non-vaccinated $L N(n=4)$ is expressed in adult and neonatal pigs. The colour scheme for the relative gene expression is as follows: The colour scheme for the relative gene expression is as follows: the yellow results are not significantly changed after vaccination and foldchange is not indicated or have a significant fold-change between 0.5 and $2 ;<0.5$ is dark green (i.e. the respective gene is down regulated); between 2 and 10 is red (i.e. the gene is moderately upregulated) or $>10$ is dark red (i.e. the gene is strongly upregulated). 


\section{Discussion}

In this study, neonatal and adult pigs were vaccinated with DMN-patches containing different TLRa in combination with iPRRSV-antigen to investigate the immune-stimulatory potential of the TLRa and whether or not these immune responses were age-dependent. We compared the NV neonatal skin to the NV adult skin to investigate if differences in skin thickness, location and quantity of APC subsets or TLR expression were associated with the higher number of up-or downregulated genes in vaccinated neonatal skin compared to adult skin. The significantly thinner skin of the neonate, mainly caused by the thinner dermis, was also observed in other porcine ${ }^{44}$ and human studies ${ }^{45}$. We consider it unlikely that the thinner skin had an impact on skin vaccination in our study, because the length of the microneedles of the DMN patch $(500 \mu \mathrm{m})$ did not exceed the thickness of the skin. We showed that the neonatal skin contained an equivalent number of DCs compared to the adult skin as was also observed in human studies ${ }^{29,46}$. However, the gene expression profile of the NV neonatal skin compared to the NV adult skin showed a lower basal expression of cell-surface markers for antigen presentation (e.g. CD86, SLA-DRB1 (MHCII)) and for DC markers (CD101 and FLT3). This indicates that in the neonatal skin DCs express fewer cell-surface markers compared to the adult skin, which could be an expression of a more immature phenotype of the neonatal skin $D C$, which could restrict $D C$ migration to the draining LN. On the other hand, the neonatal skin contained a higher number of macrophages, which was supported by the enhanced neonatal basal gene expression of CD163 compared to adults. Neonates depend more on their innate immune system ${ }^{47,48}$ and therefore the larger number of macrophages in the dermis could be consistent with a more prominent contribution to the innate immune response in the skin. This is in line with the higher percentage of monocytes in the neonatal blood compared to the adult blood ${ }^{32}$. Although dermal macrophages could be characterized as APC, they are more specialized towards phagocytosis, while DCs are more specialized in antigen presentation and activation of T-cell ${ }^{49}$. Therefore, the contribution to the enhancement of antigen presentation by the larger number of macrophages in the neonatal skin will most likely be limited. Finally, we showed that there was no difference in basal TLR gene expression within the neonatal and adult skin. Therefore, we consider it likely that the significantly larger number of dermal macrophages and differences in basal immune gene expression contributed to the age-dependent differences after skin vaccination.

In both the adult and neonatal pigs the TLR7/8a skin-vaccine induced the most prominent histopathological skin changes and skin immune response at the vaccination site. This was illustrated by the highest number of differentially expressed genes in the skin and draining LN after TLR7/8a vaccination, compared to the other TLRa-vaccines in both age-groups. We observed not only the expected upregulation of pro-inflammatory genes, which is in accordance with the leucocytes influx in the dermis, but in addition a higher expression of genes regulating APC activation and migration (CD86, CCR7 and CD69) was detected. This suggests that the local influx of leucocytes after skin vaccination 
could have contributed to APC activation after TLR7/8a vaccination. Although expression of genes associated with APCs activation was strongly induced after TLR7/8a vaccination, there was no clear indication of LC migration to the dermis. This absence of DC migration was also observed in human ex vivo skin studies ${ }^{11,50,51}$ with TLRa as an immune-stimulator. They suggested that the skin microenvironment suppressed the DC maturation/migration after TLR stimulation and that the immunosuppressive effect of IL10 interfered with the DC activation and migration ${ }^{29}$. Although no change in IL10 regulation was found in our study, the upregulation of the anti-inflammatory genes IDO1 and ILRN and downregulation of TGF $\beta$ could have contributed to the suppressive skin microenvironment after TLR7/8 vaccination and limit LC migration.

For the TLR1/2a and TLR9a vaccines the local skin responses as shown by gene expression and skin histology, were less evident than for the TLR7/8a vaccine. Interestingly, TLR9a containing vaccine resulted in more downregulated than upregulated immune response genes in the skin of both age-groups, compared to the TLR1/2a and TLR7/8 vaccines. This suggests a gene-specific profile for the TLR9a adjuvant (CpG) used in a PRRSV vaccine preparation. The gene expression by the TLR9a vaccine was more comparable to the Ag-only vaccine (without TLRa). We could therefore speculate that TLR9 stimulation was limited due to the lack of detectable TLR9 gene expression in the skin, which contributed to the observed divergent gene response from the other TLRa containing vaccines. Nonetheless, Magiri et al. showed that TLR9a (CpG) induced an upregulation of several IFN inducing genes (MX1, IRF7), chemokines (CCL5, CXCL10) and IL10 at 24h after skin vaccination in pigs ${ }^{35}$. These genes were not significantly differentially regulated in our study, except for CXCL10. This chemokine was only upregulated in neonatal pigs, but not in adult pigs. They ${ }^{35}$ suggest that CpG may promote recruitment of innate immune cells and Th1 type cytokine production, but this is not supported from our study. However, they used 4-weeks-old pigs, less CpG $(10 \mu \mathrm{g})$ and an intradermal injection as the route of administration and these differences in study design could have led to different results as compared to what we observed. In a murine study ${ }^{52}$ TLR9a (CpG) induced an upregulation of cytokine and IFN-related genes when they compared this to treatment TLR7/8a (R848) and TLR1/2a (Pam3Cys) at $6 \mathrm{~h}$ after i.m. administration of an influenza vaccine. In contrast to the skin histopathology after TLR7/8a vaccination in our study, the injected muscles in this mice study showed no clear influx of leucocytes with the TLR7/8a and TLR9a containing vaccines. However, they observed this leucocyte influx in the muscle with the TLR1/2a vaccine. Another murine study ${ }^{53}$ showed that topical and subcutaneous delivery of TLR7/8a R848, but not i.m. delivery enhanced the immune response. These results suggest that the route of delivery could be an important prerequisite for the induction of local and systemic immune responses when using TLRa as immune-stimulator in vaccines and highlights the potential differences between mice and pigs.

The early immune response in the draining $L N$, is essential to induce and modulate the subsequent adaptive immune response. In all draining LNs, except for the LNs of adult 
pigs vaccinated with TLR9a, the gene expression of CCR7 was significantly upregulated. This suggests that the draining $L N$ had an increased number of activated DCs expressing CCR7. On the other hand, the fold-change in gene induction in the draining LN was clearly lower compared to the genes that were induced in the skin after vaccination. The duration needed for $\mathrm{LN}$ activation after skin vaccination could be the cause of this lower gene induction in the draining LNs. Significant gene activation in the draining LN with CpG vaccination in goats ${ }^{54}$ required $72 \mathrm{~h}$. On the other hand, several studies with pigs ${ }^{55-57}$ and neonatal primates ${ }^{58}$ reported significant gene upregulation in the draining $\mathrm{LN}$ already within $24 \mathrm{~h}$ following skin and i.m. vaccination. The overall lower gene induction of the draining LNs in our study could suggests that the development of a specific adaptive immune response after skin vaccination was hampered as was shown in a PRRSV vaccine study where we used similar skin vaccines ${ }^{38}$.

Differences in basal gene expression in the neonatal control LN compared to adult control LNs could indicate a difference in immune development between adult and neonatal LNs contributing to age-dependent differences in gene induction after skin vaccination. Genes involved in B-cell activation (CD40/CD69) showed a lower basal expression in the neonatal LN compared to the adults, which is consistent with the limited protective B-cell responses described for neonates ${ }^{25,59}$. Additionally, lower gene expression of Th1 cytokines IFNY and IL $12{ }^{60}$ in the neonatal LN support the limited neonatal cellular immune responses. These differences could limit the development of a specific protective immune response in neonatal pigs after vaccination.

The placebo patch was used to investigate the effects of mechanical stimulation and induced predominantly downregulation of pro-inflammatory genes in both adult and neonatal pigs compared to the NV pigs. Another porcine study showed also that placebo intradermal injection did not induce genes at $24 \mathrm{~h}$ after vaccination, although at $2 \mathrm{~h}$ after vaccination upregulation of pro-inflammatory genes (IL8, IL1 $\beta$ ) and chemokines (CCL2, CCL3 and CCL20) was found ${ }^{61}$. Therefore, it is possible that the downregulation of e.g. CCL3 and DEFB1 observed in our study could have been preceded by an upregulation of these genes. Interestingly, more genes (e.g. chemokines CCL20 and CXCL10 in the APC cluster) were upregulated in the neonatal pigs compared to the adult pigs after placebo patch vaccination, indicating that the neonatal skin was more responsive to the mechanical stimulation than the adult skin.

The antigen-only $(\mathrm{Ag})$ vaccination, which was administered in neonates only, induced minimal changes in gene expression in the vaccinated skin when compared to the vaccines in conjunction with the different TLRa. However, the changes in gene expression induced by the Ag-vaccine in the draining $\mathrm{LN}$ were comparable to the vaccines containing TLRa. This suggests that the TLRa were especially important for the local skin immune responses and that the Ag had a limited contribution to the local immune response in the skin. 
This study investigated the skin as vaccine administration route in neonatal and adult pigs. We showed induction of significant immune responses in both neonatal and adult skin after application of DMN-patches loaded with iPRRSV and different TLRa. In both age-groups, skin vaccination with TLR7/8a induced the most prominent early immune responses compared to other TLRa vaccines, especially in the skin. However, agedependent differences in immune responses should be considered when developing skin vaccines for pigs in different age-groups.

\section{Conflict of interest statement}

Anne Moore is an inventor of patents that have been or may be licensed to companies developing microneedle-based products. This potential competing interest has been disclosed and is being managed by University College Cork. Dennis McDaid is Chief Operating Officer and current director and owns stock in Xeolas Pharmaceuticals Limited. Joanne McCaffrey and Damien Collins are former employees of Xeolas Pharmaceuticals Limited and had no financial or other competing interests. The other authors have no competing interest to declare.

\section{Acknowledgments}

Corry Dolstra, Rob Zwart and Anja Taverne-Thiele are acknowledged for their technical assistance with the immunohistochemistry. Lisette Ruuls and Karin Tarp are acknowledged for their assistance with the qPCR. Conor O'Mahony at the Tyndall National Institute, UCC is acknowledged for supplying PDMS moulds. This project has received funding from the European Union's Horizon 2020 Programme for research, technological development and demonstration under the Grant Agreement $n^{\circ} 633184$. This publication reflects the views only of the author, and not the European Commission (EC). The EC is not liable for any use that may be made of the information contained herein. 


\section{Supplementary data}

Table S1 A: Grading skin histology in skin biopsies.

\begin{tabular}{|c|c|c|c|c|}
\hline score & \multicolumn{4}{|c|}{ Histologic evaluation of skin biopsies } \\
\hline & \multicolumn{4}{|l|}{ Influx and distribution of inflammatory cells in skin } \\
\hline 0 & \multicolumn{4}{|l|}{ No increase } \\
\hline 1 & \multicolumn{4}{|l|}{ Mild increase only perivascular } \\
\hline 2 & \multicolumn{4}{|l|}{ Increase not restricted to perivascular, extending in the dermis } \\
\hline \multirow[t]{2}{*}{3} & \multicolumn{4}{|c|}{ More diffuse dermal distribution- occasionally effacing epidermal/dermal junction } \\
\hline & \multicolumn{4}{|l|}{ Hyperaemia blood vessels/ hemorrhage } \\
\hline 0 & \multicolumn{4}{|l|}{ No hyperaemia } \\
\hline 1 & Mild hyperaemia in superficial dermis & \multirow{2}{*}{\multicolumn{3}{|c|}{$\begin{array}{l}\text { Final histology scoring } \\
\text { including all parameters } \\
\text { (max } 7)\end{array}$}} \\
\hline 2 & Clear hyperaemia, occasional hemorrhage & & & \\
\hline & Dermal edema & $\mathbf{0}$ & 0 & 1 \\
\hline 0 & No edema & 1 & $>1$ & 3 \\
\hline 1 & Mainly perivascular edema & 2 & $>3$ & 5 \\
\hline 2 & Collagen vessels are multifocally dispersed by clear empty spaces & 3 & $>5$ & 7 \\
\hline
\end{tabular}

All skin biopsies (haematoxylin and eosin stain (HE)) stain were graded semi-quantitatively for inflammatory cells (0-3), hyperaemia (0-2) and dermal edema (0-2). The overall histology score (0-7) was translated in no changes (0), mild changes (1), moderate changes (2) and severe changes (3) after skin vaccination.

Table S1B: Grading CD1a expression in skin biopsies.

\begin{tabular}{ll}
\hline score & Evaluation CD1a expression in skin biopsies \\
\hline & Epidermis \\
\hline $\mathbf{0}$ & No staining \\
\hline $\mathbf{2}$ & Only a few cells stain positive \\
\hline $\mathbf{3}$ & Dispersed irregular staining \\
\hline $\mathbf{0}$ & Dositive staining regular dispersed over basal layer epidermis \\
\hline $\mathbf{1}$ & Do staining \\
\hline $\mathbf{2}$ & Dispersed staining in dermis \\
\hline $\mathbf{3}$ & Regular positive staining around most blood vessels \\
\hline
\end{tabular}

The number of positive CD1a staining cells in the epidermis (0-3) and dermis (0-3) were semiquantitatively evaluated in skin biopsies of non-vaccinated skin and vaccinated skin. 
Table S2: Overview cell-surface markers immunofluorescence microscopy.

\begin{tabular}{|c|c|c|c|c|}
\hline \multirow[b]{2}{*}{1} & \multicolumn{2}{|c|}{ Primary antibody (isotype) } & \multicolumn{2}{|c|}{ Secondary antibody (isotype) } \\
\hline & CD1a (lgG2a) & $1: 125$ & AF647 (IgG2a) & $1: 200$ \\
\hline & CD163 (lgG1) & $1: 200$ & AF568 $(\lg G 1)$ & $1: 400$ \\
\hline \multirow[t]{2}{*}{2} & CD1a (lgG2a) & $1: 125$ & AF647 (lgG2a) & $1: 200$ \\
\hline & MHCII (IgG1) & $1: 1000$ & AF568 $(\lg G 1)$ & $1: 400$ \\
\hline
\end{tabular}

\begin{tabular}{|c|c|c|c|c|c|c|c|}
\hline Cluster & $\begin{array}{l}\text { Gene symbol (assay } \\
\text { number) }\end{array}$ & Gene name & Forward primer sequence (5' to $\left.3^{\prime}\right)$ & Reverse primer sequence ( $\left(5^{\prime}\right.$ to $\left.3^{\prime}\right)$ & $\begin{array}{l}\text { Amp } \\
\text { length }\end{array}$ & gene ontogeny (GO) & Reference \\
\hline \multirow{6}{*}{ 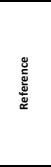 } & АСтB (6) & Actin, Beta & F: CTACGTCGCCCTGGACTTC & R: GCAGCTCGTAGCTCTTCTCC & 76 & reference gene & \\
\hline & $\operatorname{Bz} \mathrm{M}(7)$ & Beta-2-Microglobulin & F: TGAAGCACGTGACTCTCGAT & R: CTCTGTGATGCCGGTTAGTG & 70 & reference gene & \\
\hline & GAPDH (20) & Glyceraldehyde-3-phosphate dehydrogenase & F: ACCCAGAAGACTGTGGATGG & R: AAGCAGGGATGATGTTCTGG & 79 & reference gene & \\
\hline & HPRT1 (25) & Hypoxanthine phosphoribosyl-transferase 1 & F: ACACTGGCAAAACAATGCAA & R: TGCAACCTTGACCATCTTTG & 71 & reference gene & \\
\hline & PPIA (154) & peptidylprolyl isomerase A (cyclophilin A) & F: CAAGACTGAGTGGTTGGATGG & R: TGTCCACAGTCAGCAATGGT & 138 & reference gene & \\
\hline & YWHAE (156) & $\begin{array}{l}\text { Tyrosine 3-monooxygenase/tryptophan 5- } \\
\text { monooxygenase }\end{array}$ & F: GCTGCTGGTGATGATAAGAAGG & R: AGTTAAGGGCCAGACCCAAT & 124 & reference gene & \\
\hline \multirow{25}{*}{ 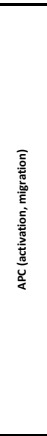 } & CCL19 (908) & C-c Motif Chemokine Ligand 19 & $\mathrm{~F}:$ CTGGACTTCTCCTGCTCTGG & R: AAAGGCTCGAACCAGATTCC & 97 & chemokine DC attraction & \\
\hline & CCL2 (117) & C-c Motif Chemokine Ligand 2 & F: GCAAGTGTCCTAAAGAAGCAGTG & R: TCCAGGTGGCTTATGGAGTC & 103 & chemokine DC attraction & Leluduec 2016 \\
\hline & CCL20 (995) & C-C Motif Chemokine Ligand 20 & F: CTGCAGCAAGTCAGAAGCAG & R:GCTGGGGAAGCCCATGATA & 95 & chemokine DC attraction & Auray 2013 \\
\hline & CCL3 (236) & C-C Motif Chemokine Ligand 3 & $\begin{array}{l}\text { F: CTCTGCAGCCAGGTCTTCTC } \\
\text { F CTCAG }\end{array}$ & R: CTACGAATTTGCGAGGAAGC & 97 & $\begin{array}{l}\text { chemokine } \mathrm{DC} \text { a attraction } \\
\text { chemokine DC }\end{array}$ & Magiri 2016 \\
\hline & $\operatorname{ccl}(121)$ & C-C Motif Chemokine Ligand 5 & F: CTCCATGGCAGCAGTCGT & R: AAGGCTTCCTCCATCCTAGC & 121 & & Leluduec 2016 \\
\hline & 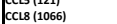 & $\begin{array}{l}\text { Chemokine ligand } 8 \\
\text { Chgand 5 }\end{array}$ & F: GCCAGATTCAGTCTCCATCC & R: AGGGGATCTTTCCATTGACC & 62 & chemokine DC attraction & $\begin{array}{l}\text { Leleluduec } 2016 \\
\text { Moreno } 2013\end{array}$ \\
\hline & CCR7 (607) & Chemokine (C-C Motif) Receptor 7 & F: TCCACGTCTGCAAACTCATC & R: GTCGATGCTGATGCAGAGAA & 83 & DC migration to draining Inn & \\
\hline & CCR7 (608) & Chemokine (C-C Motif) Receptor 7 & F: GCCATGAGCTTCTGCTACCT & R: CCTTGTTGCGCTCGAAGT & 70 & DC migration to draining Inn & \\
\hline & CD101(572) & CD101 & F: ATTGAAACTCAGGCCCACAG & R: CCTGGCTGTGTTCTGTAGCA & 80 & DC marker & \\
\hline & $\begin{array}{l}\text { CD14 (8) } \\
\text { (8) }\end{array}$ & CD14 & F: AAGCTCACCGTGCTTGATCT & R: CCTTCCAGGGTCAGGTCAT & 92 & monocyte marker & \\
\hline & CD163 (150) & CD163 & F:CACATGTGCCAACAAAATAAGAC & R: CACCACCTGAGCATCTTCAA & 130 & macrophage marker & \\
\hline & CD1A (556) & CD1a & F: TAGGAGGCCAGGACATCATC & R: GGCACAATCACTGCCAATAA & 76 & DC marker & \\
\hline & CD209 (586) & CD209 & F: CGGAGCAGAAATTCCTGAAG & R: CATTGCCAGGAACCTTCATT & 94 & DC marker & \\
\hline & CD40 (9) & $\mathrm{CD} 40$ & F: TGAGAGCCCTGGTGGTTATC & R: GCTCCTTGGTCACCTTTCTG & 90 & antigen presentation & \\
\hline & 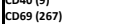 & CD69 & F: CCAAAGCTTCTGCTCCAAAC & R: GTGTTCAGCTCTCCCCACAT & 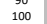 & $\begin{array}{l}\text { antigen presentation } \\
\text { antigen presentation }\end{array}$ & \\
\hline & CD80 (560) & CD80 & F: CGCACCTTCACTGATGTCAC & R: CACAGGTGTAGGTGCCATTG & 82 & antigen preentation & \\
\hline & CD86 (560) & CD86 & F: CATCGTCTGTGTCCTGCAAC & R: CACAGGTGGCTTTGCATCTA & 82 & antigen presentation & \\
\hline & CxCL10 (111) & Chemokine (C-X-C Motif) Ligand 10 & F: CCCACATGTTGAGATCATTGC & R: GCTTCTCTCTGTGTTCGAGGA & 141 & chemotraction DC & \\
\hline & CXCR4 (575) & Chemokine (C-X-C Motif) receptor 4 & F: CTGCTGGCTGCCATACTACA & $\mathrm{R}:$ TCAAACTCACACCCTTGCTG & 81 & receptor LC for migration to dermis & Forster 2012 \\
\hline & FLT3 (580) & CD135/ Fms-Related Tyrosine Kinase 3 & F: ATGGATCAGCCATTTIACGC & R: CGTTTCCTGGAGTCAAAAGC & 77 & DC marker (bone marrow derived) & \\
\hline & MMP2 (332) & Matrix Metallopeptidase 2 & F:TCGCTGGAGATAAGTTCTGGAG & R: GGCGTCTGCAATGAGCTT & 80 & migration $D C$ & Magiri 2016 \\
\hline & MMP9 (335) & Matrix Metallopeptidase 9 & F: ACACACGACATCTTCCAGTACC & R: GTCCACCTGATTCACCTCGT & 96 & migration DC & Forster 2012 \\
\hline & Mx1 (501) & Interferon-induced GTP-binding protein M×1 & F: GCCGAGATCTTTCAGCACCT & R: CGGAGGATGAAGAACTGGATGA & 95 & migration $D C$ & \\
\hline & & Major Histocompatibility Complex, Class II, DR & & & & & \\
\hline & SLA-DRB1 (593) & Beta 1 & F: TGACGGTGTATCCTGCAAAG & R: GTAGAACCCGGTCACAGAGC & 74 & antigen presenting & \\
\hline \multirow{18}{*}{ 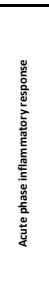 } & $c 3(10)$ & Complement Component 3 & F: ATCAAATCAGGCTCCGATGA & R: GGGCTTCTCTGCATTTGATG & 76 & acute phase protein & \\
\hline & CASP1 (184) & Caspase 1 & F: GAAGGACAAACCCAAGGTGA & R: TGGGCTTTCTTAATGGCATC & 147 & inflammasome & \\
\hline & CASP3 (383) & Caspase 3 & $\begin{array}{l}\text { F: CTGGCAAACCCAAACTTTTC } \\
\text { F }\end{array}$ & R: GTCCCACTGTCCGTCTCAAT & 79 & inflammasome & \\
\hline & CRP (12) & C-Reactive Protein & $\mathrm{F}:$ GGTGGGAGACATTGGAGATG & R: GAAGGTCCCACCAGCATAGA & 85 & acute phase protein & Forster 2012 \\
\hline & DEFB1 (779) & Defensin Beta 1 & E: ПTCCTCCTCATGGTCCTGTT & R: CATCTTTGGAGCACACTTGC & 114 & neutrophil function & Joronen 2014 \\
\hline & GZMA (758) & granzyme A & F: AAGGGGATCTTCAGCTGCTT & R: GGGGTTCGACATCTITTCCT & $\begin{array}{l}114 \\
99\end{array}$ & neutrophil function & \\
\hline & GZMB (208) & Granzyme B & F: CCAGGACCAGGATAATCGAA & R: GGGTGACGITGATTGAGCT & 101 & neutrophil function & \\
\hline & ICAM1 (369) & Intercelluluar Adhesion Molecule 1 & F: AAGCTTCTCCTGCTCTGCTG & R: GGGGTCCATACAGGACACTG & 89 & extravastion leucocytes & \\
\hline & $\mid 118(36)$ & Interleukin 8 & F: TTGCCAGAGAAATCACAGGA & R: TGCATGGGACACTGGAAATA & 78 & pro-inflammatory/chemokine CXCL8 & \\
\hline & 1118 (37) & Interleukin 8 & F: GAAGAGAACTGAGAAGCAACAACA & R: TGTGTTGGCATCTTTACTGAGA & 99 & pro-inflammatory/chemokine CXCL8 & \\
\hline & NOS2 (102) & Nitric Oxide Synthase 2 & F: CAGCCCAAGGTCTATGTTCAAG & R: ATAGAGGTGGCCTTGCTCCT & 90 & & \\
\hline & SAA (158) & Serum Amyloid A & F: GCTAAAGTGATCAGCGATGC & R: AGTGGTTGGGGTCCTTGC & 145 & acute phase protein & Sedlak 2014 \\
\hline & SELE (106) & Selectin $\mathrm{E}$ & F:GGATGCTGCCTACTTGTGAAG & R: CAGGAGCCAGAGGAGAAATG & 113 & neutrophil & \\
\hline & SELP (461) & Selectin $P$ & F: AGTATGCAGAGCTGTCAAATGC & R: GAAGCTGCAGGTIGATCCAT & 106 & neutrophil & \\
\hline & TNF (125) & Tumor Necrosis Factor & F:CACGTTGTAGCCAATGTCAAAG & R: GAGGTACAGCCCATCTGTCG & 129 & acute phase inflammation & \\
\hline & $\operatorname{TNF}_{\operatorname{TNF}}^{1}(74)$ & Tumor Necrosis Factor & F: CCCCCAGAAGGAAGAGTITC & R: CGGGCTTATCTGAGGTTTGA & 92 & acute phase inflammation & \\
\hline & TPS3 (493) & tumor protein $\mathrm{p} 53$ & F: TGAATGACGCCTTGGAGCTG & R: ITATGGGGGGAGGGAGACT & 113 & & \\
\hline & VCAM1 (346) & Vascular Cell Adhesion Molecule 1 & F: CTTGACGTGAAAGGAAGAGAAAG & R: GGATGCACAATAGAGCACGA & 72 & extravastion & Ahlberg 2012 \\
\hline \multirow{5}{*}{ 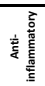 } & 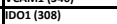 & Indoleamine 2,3-dioxygenase 1 & F: GGGCCCATGACTTACAAGAA & R: TITCCACCAATAGCGAAACC & 94 & promotes Treg & \\
\hline & $\mid 110(133)$ & Interleukin 10 & F: TACAACAGGGGCTTGCTCTT & R: GCCAGGAAGATCAGGCAATA & 110 & cytokine, anti-inflamm & Akira 2004 \\
\hline & | 1110 (39) & Interleukin 10 & $\mathrm{~F}:$ TIAAGGGTTACCTGGGTTGC & R: CTCTCTGCCTTCGGCATTAC & 80 & cytokine, anti-inflamm & \\
\hline & |LIRN (142) & Interleukin 1 Receptor Antagonist & F: TGCCTGTCCTGTGTCAAGTC & R: GTCCTGCTCGCTGTCTTTC & 90 & IL- $1 \alpha / \beta$ receptor antagonist & \\
\hline & TGFb (860) & Transforming growth factors beta & F: GCAAGGTCCTGGCTCTGTA & R: TAGTACACGATGGGCAGTGG & 97 & anti-inflammatory cytokine & Forster 2012 \\
\hline
\end{tabular}

\section{Table S3: Gene selection and primer overview.}

Overview of all selected genes divided into different immune response clusters. For each gene the primer sequence, amplicon (amp) length, and gene function, are indicated. 


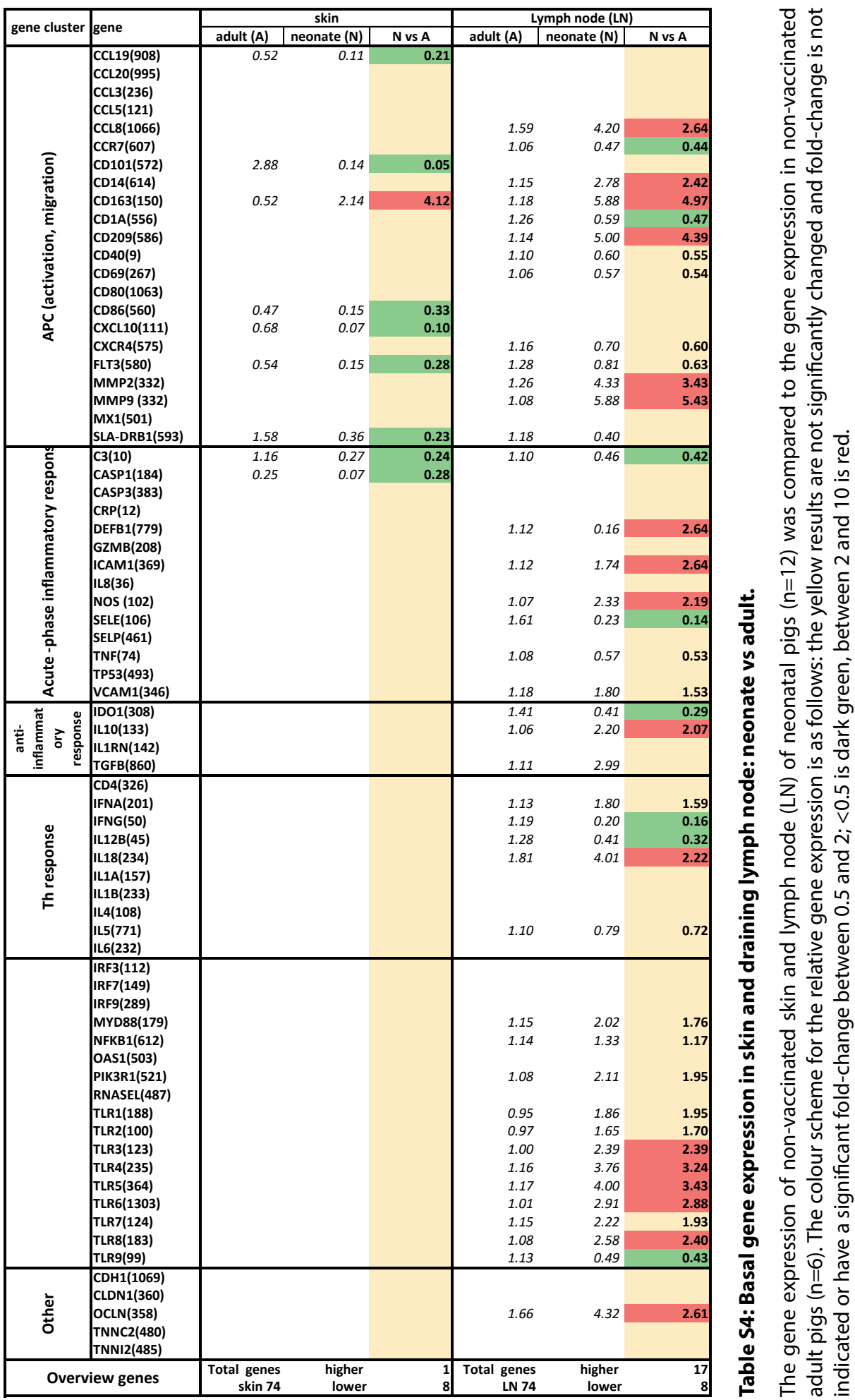




\section{Supplementary figures}

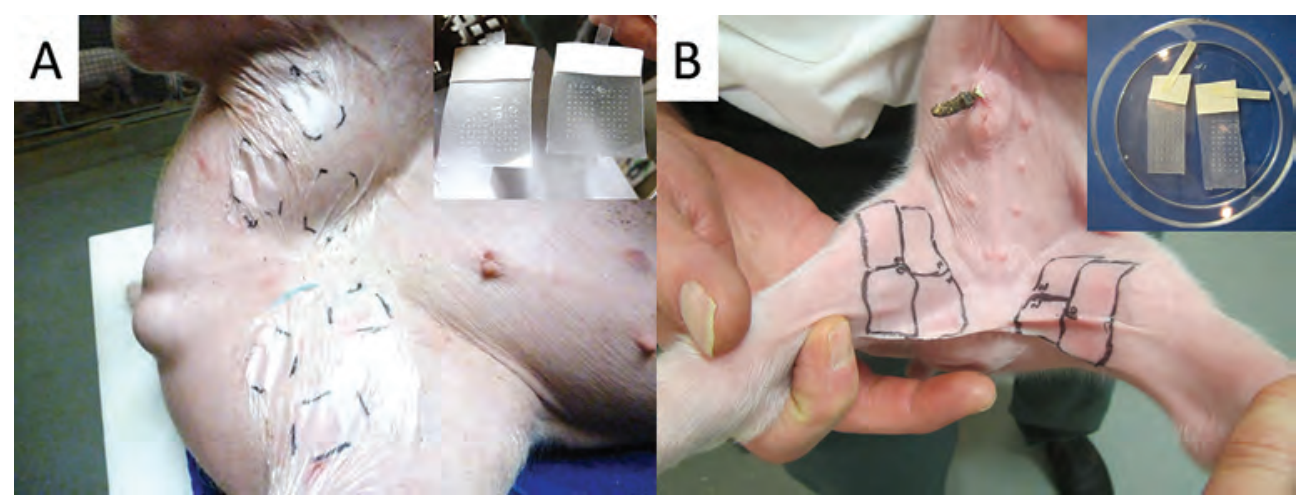

Figure S1: DMN-patch administration on adult and neonatal pigs.

(A) Adult pig, each leg contains one specific vaccine dose with 200 DMN divided over 2 separate patches and one placebo patch with 100DMN; inset DMN-patches for adult pig; (B) Neonatal pig, each leg contains one specific vaccine dose with 200 DMN divided over 4 separate patches; inset DMN-patches for neonatal pig. 


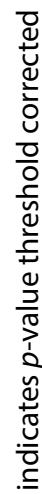
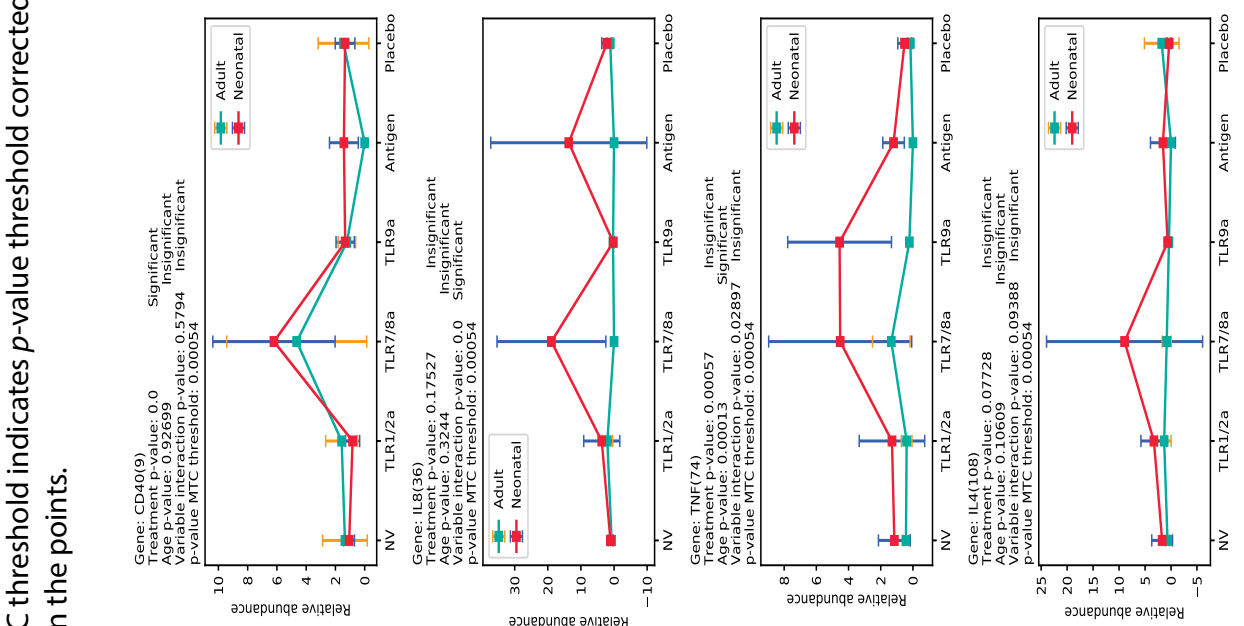

产

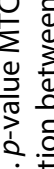
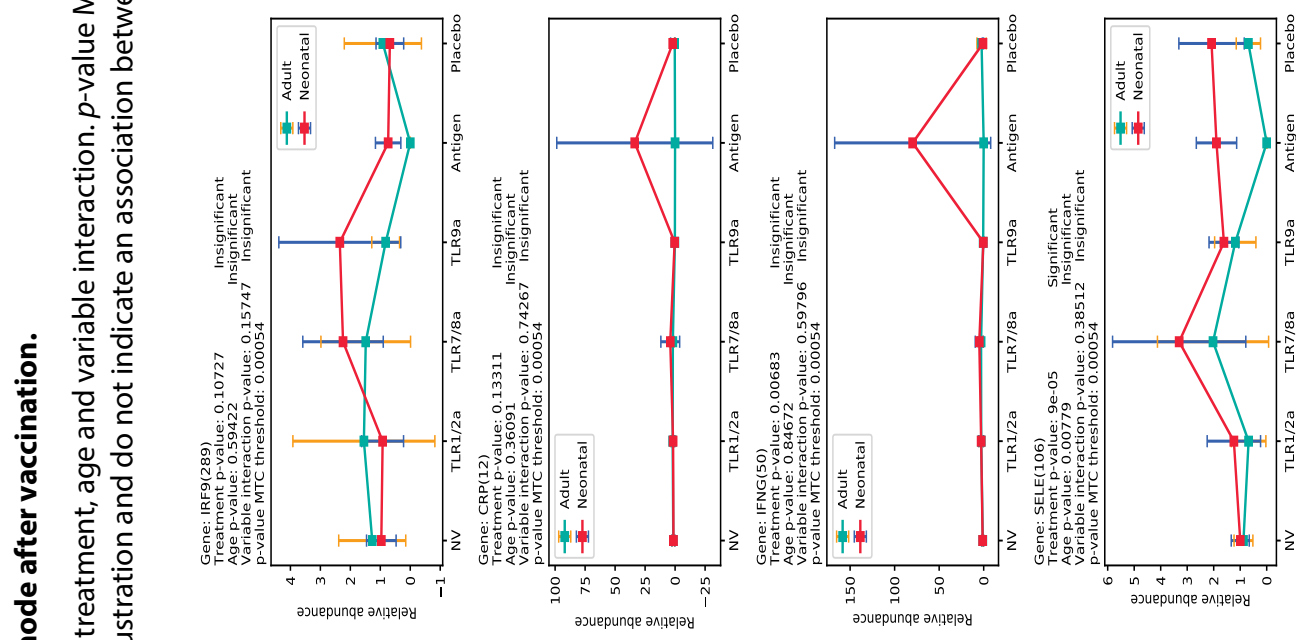

흔

은 $\frac{0}{2}$
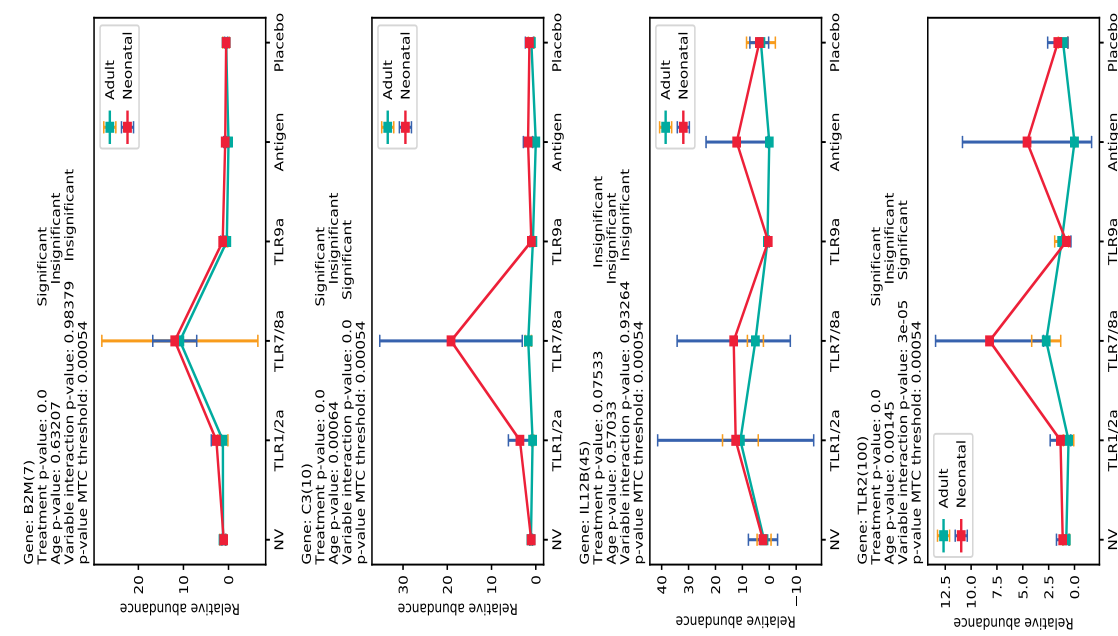

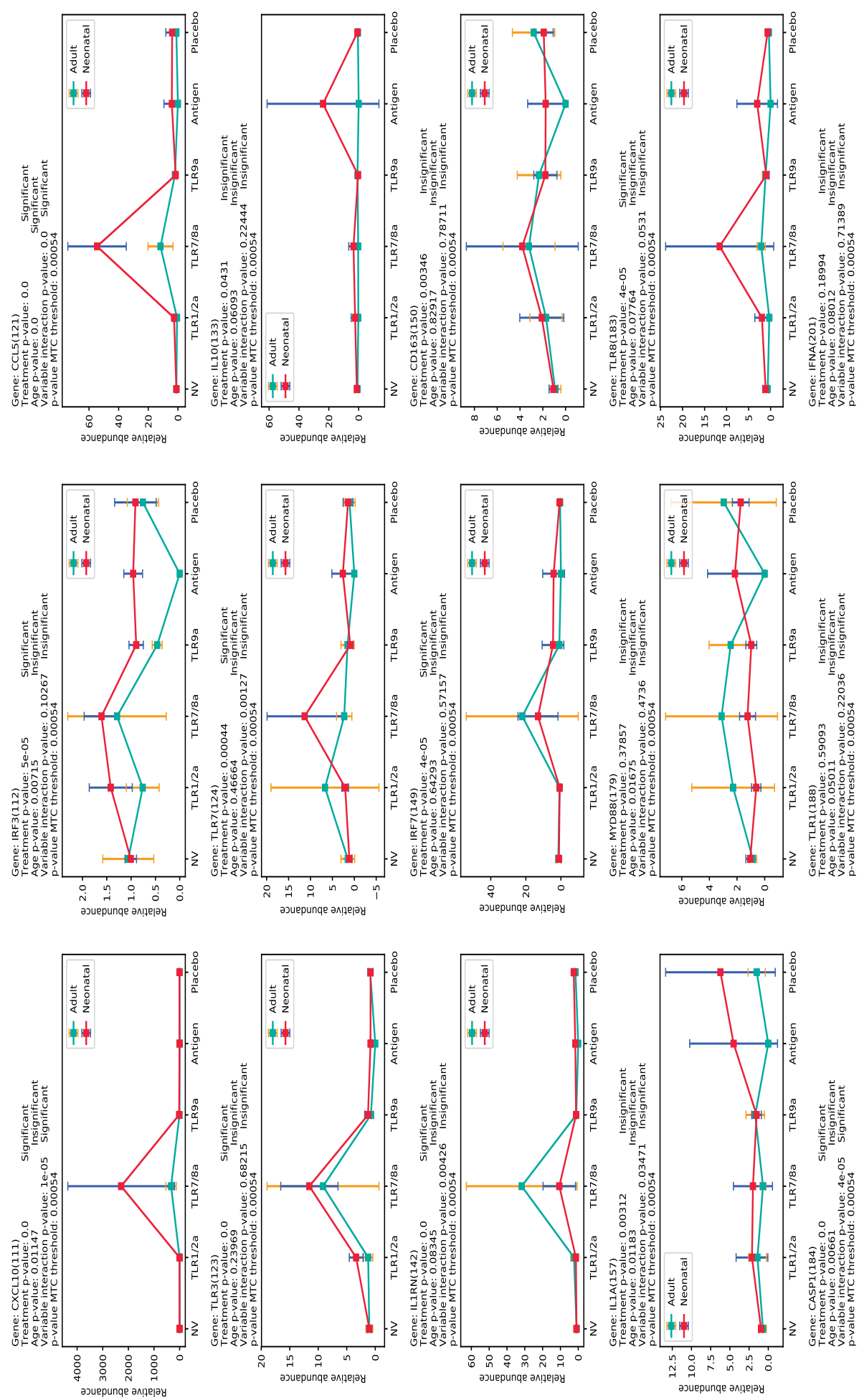

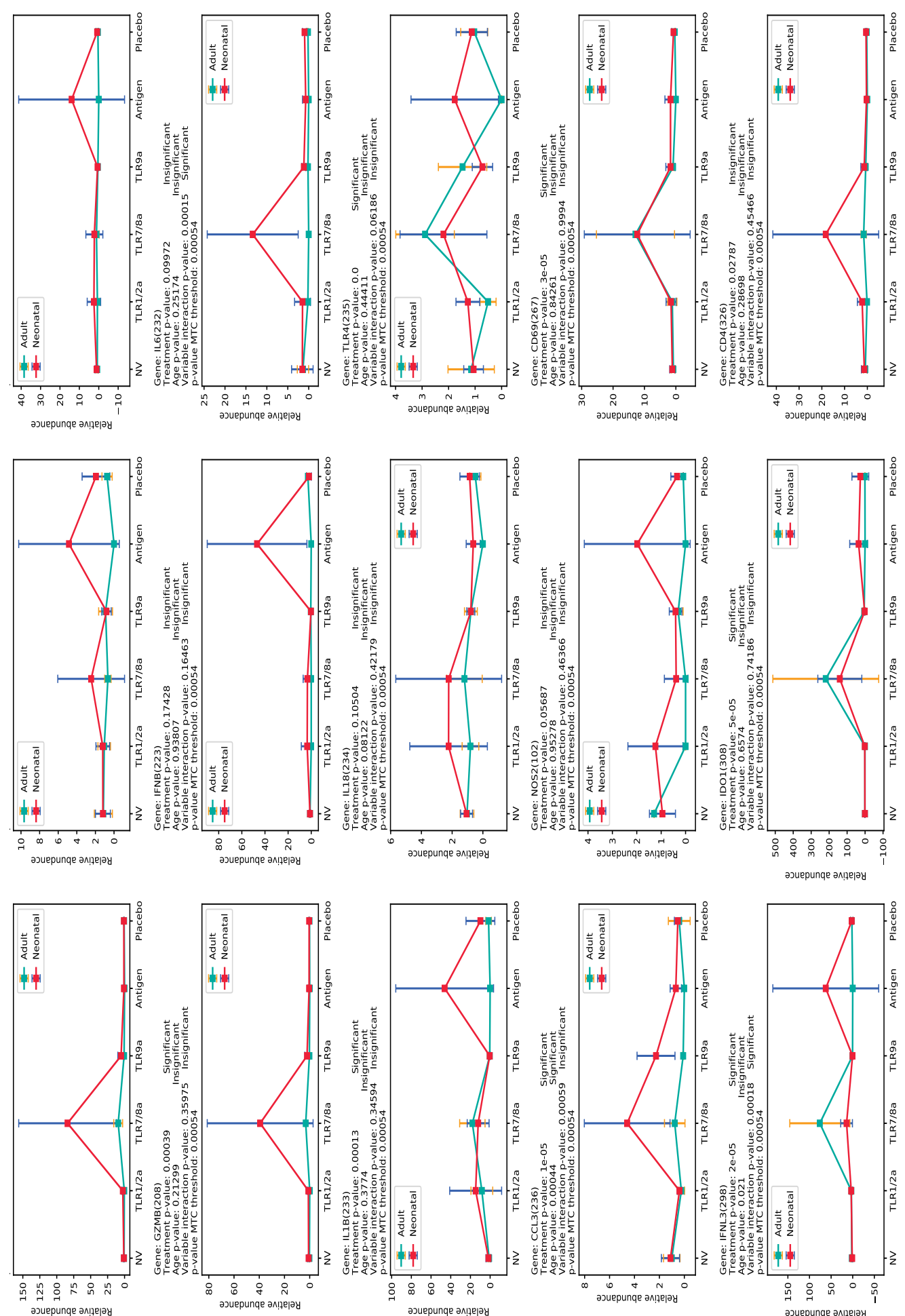

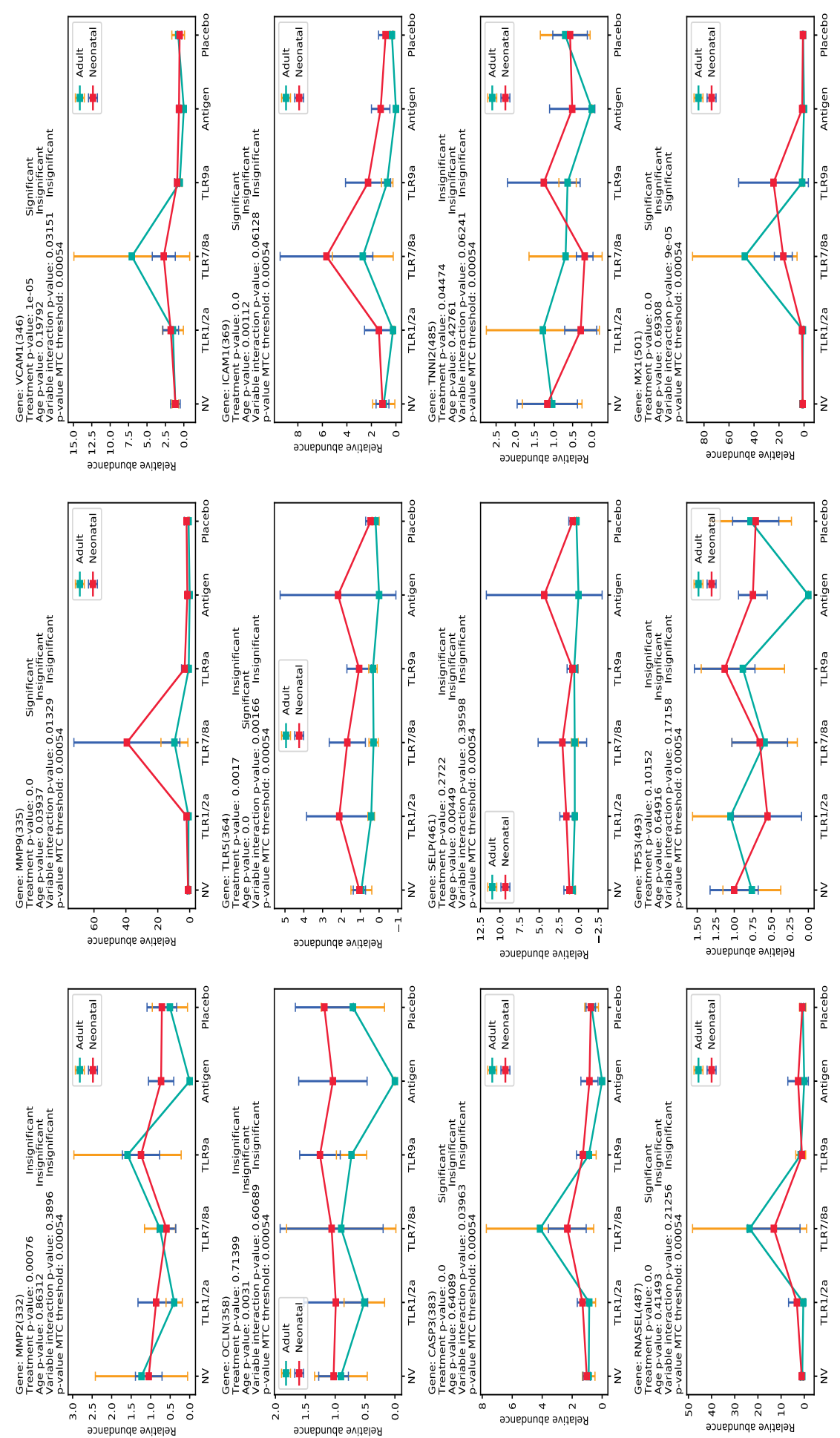

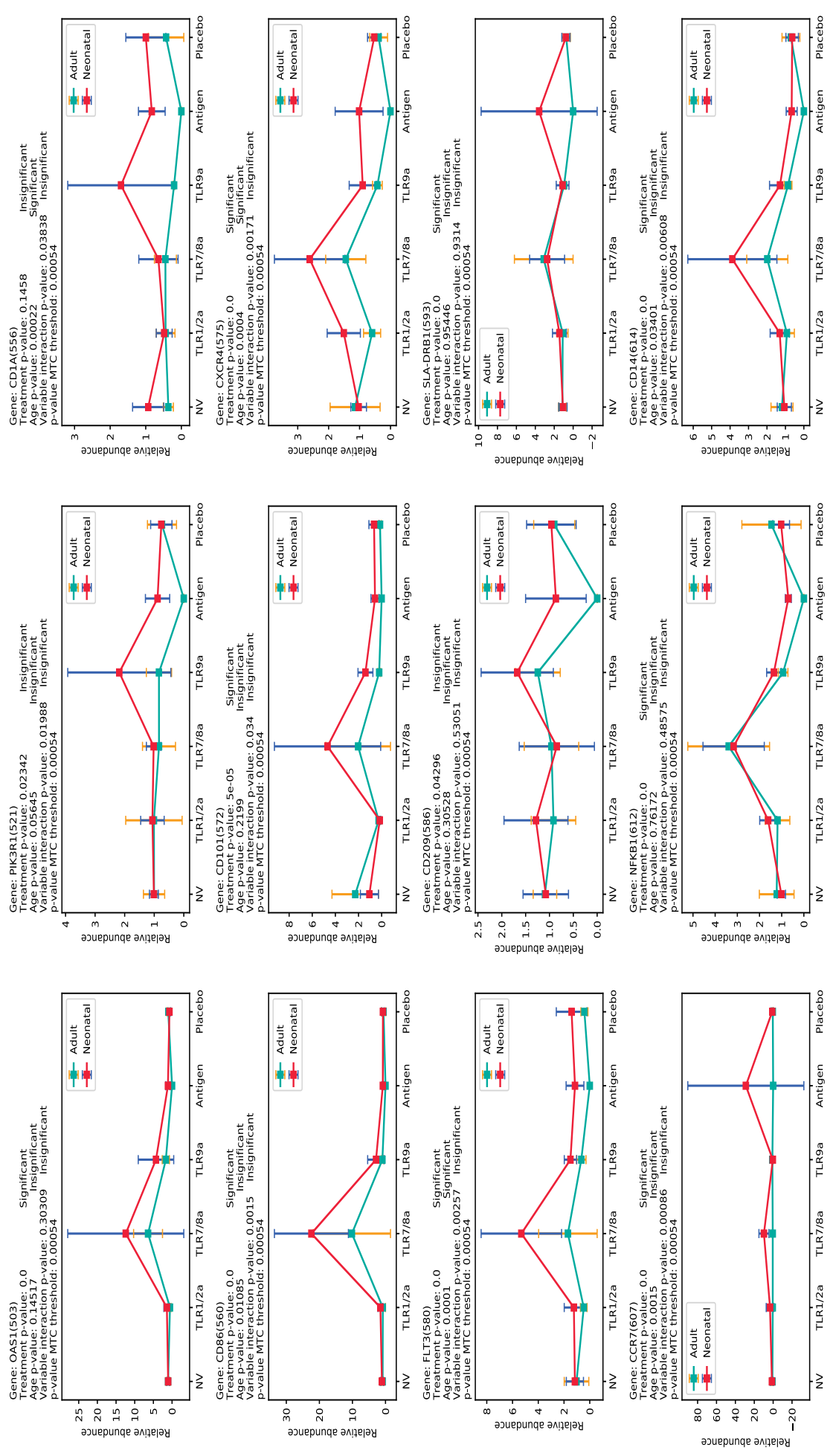

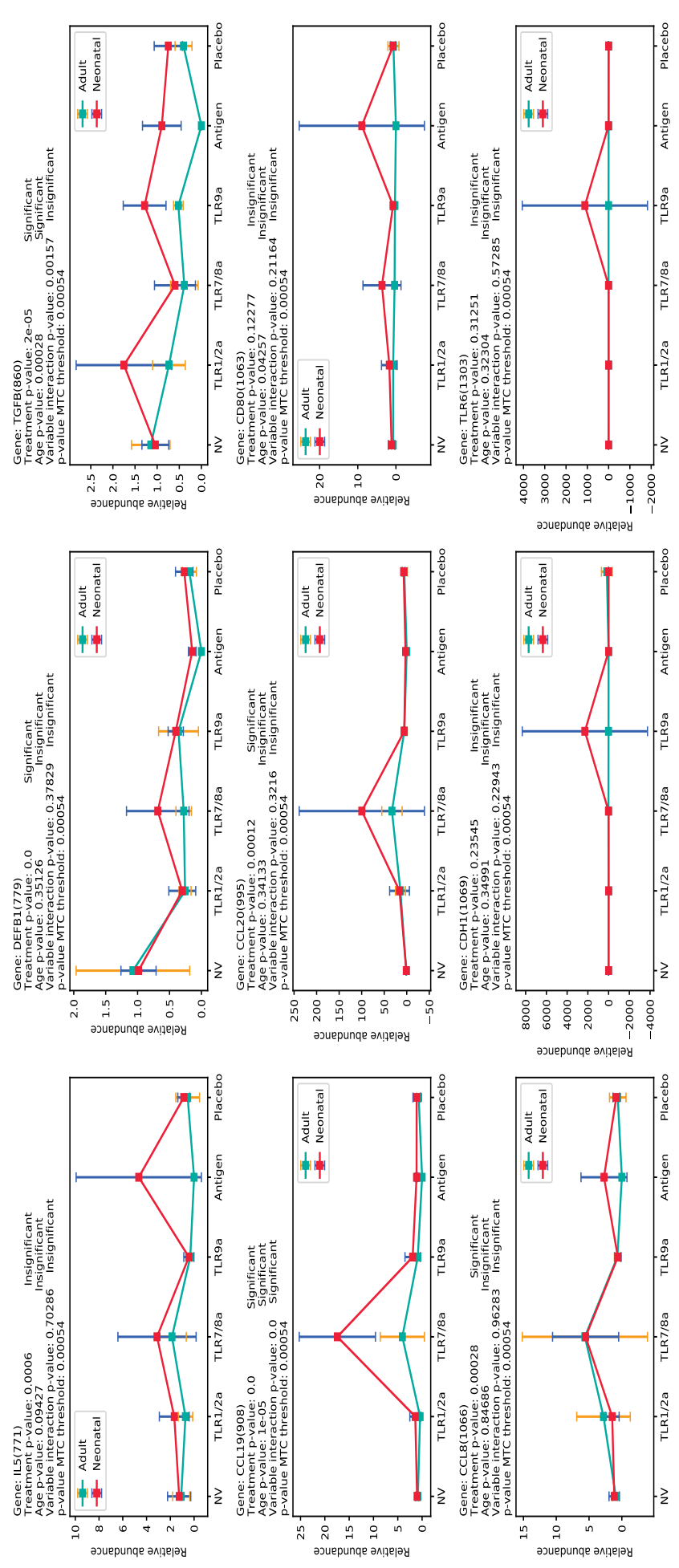


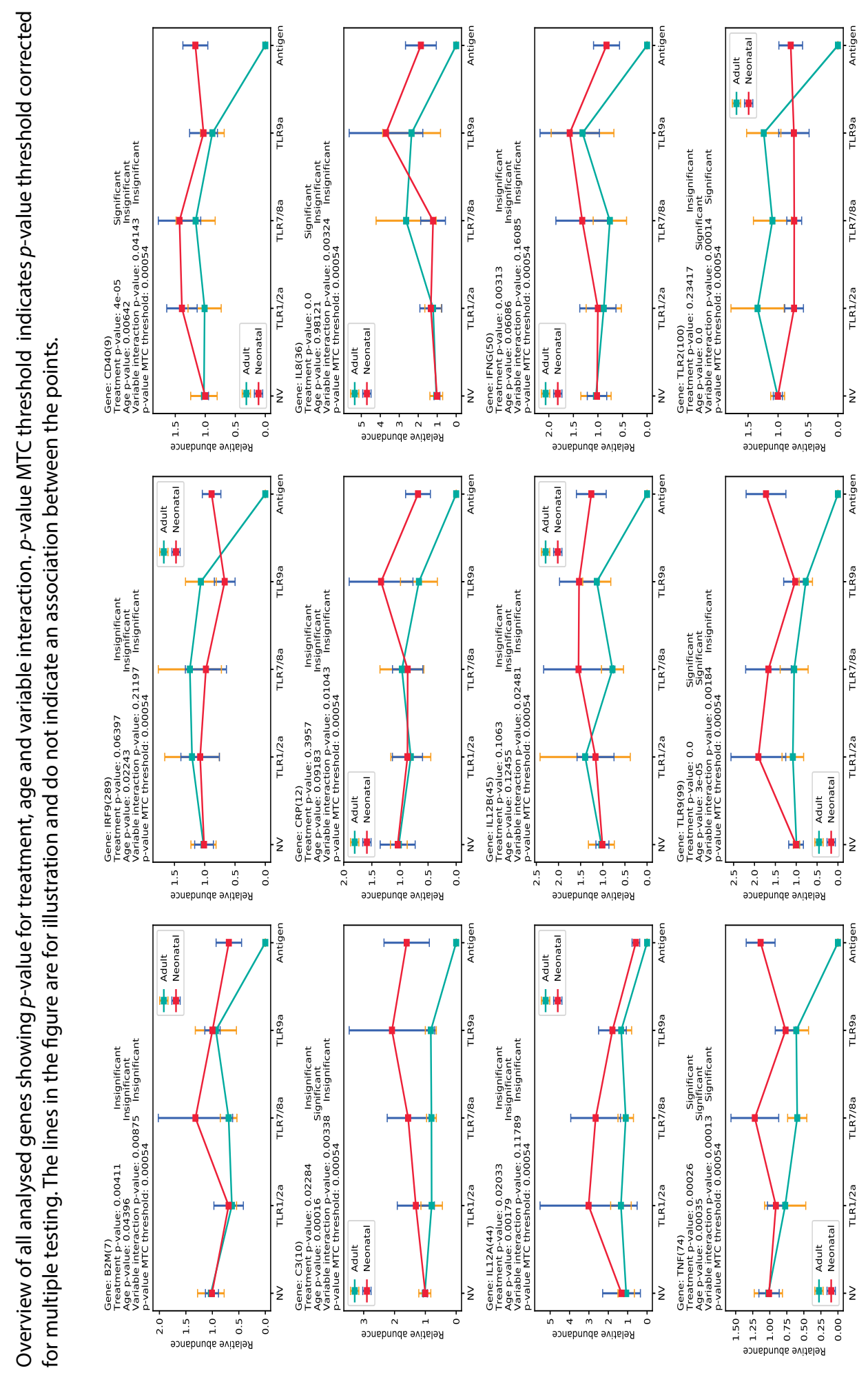



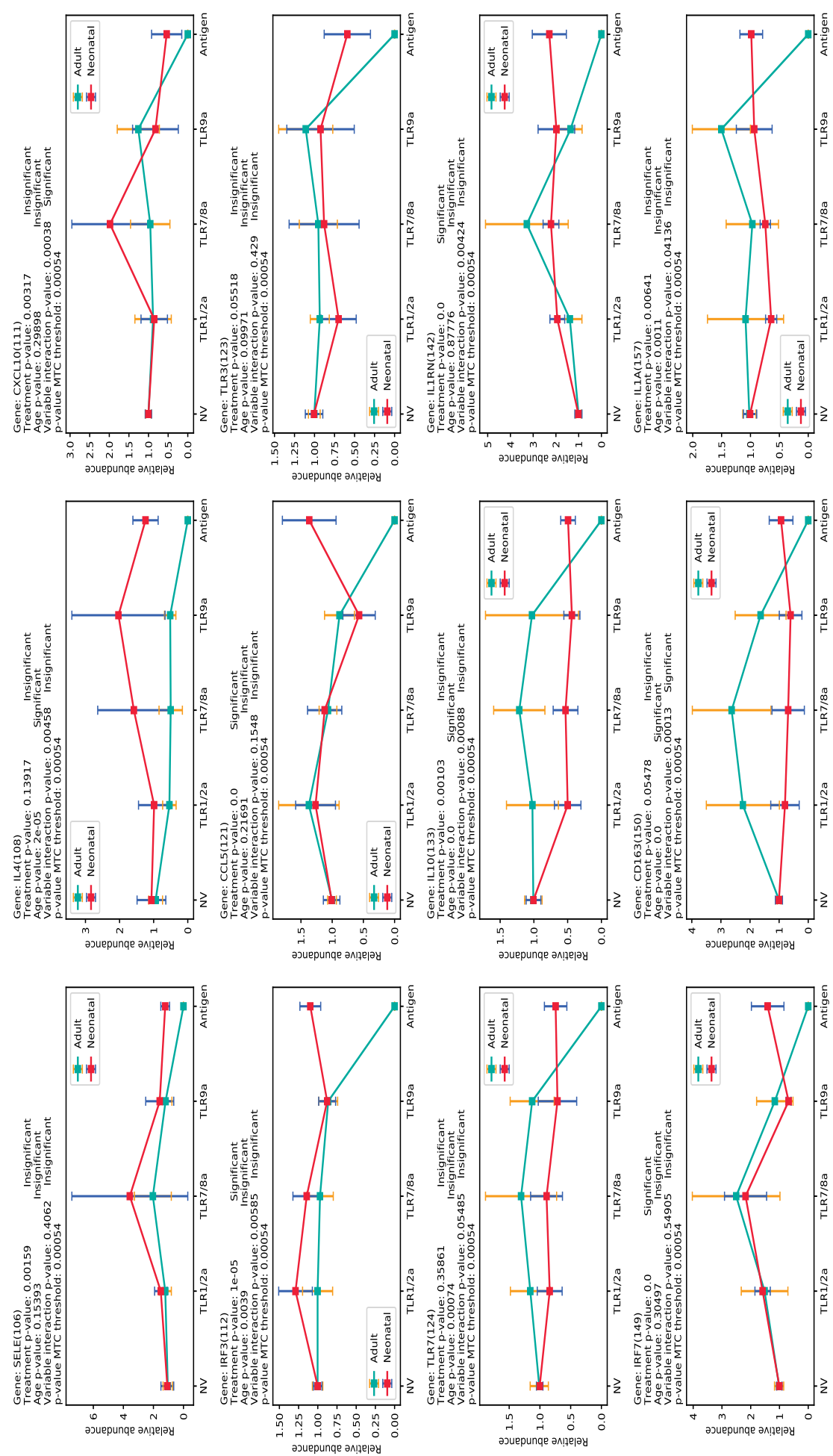

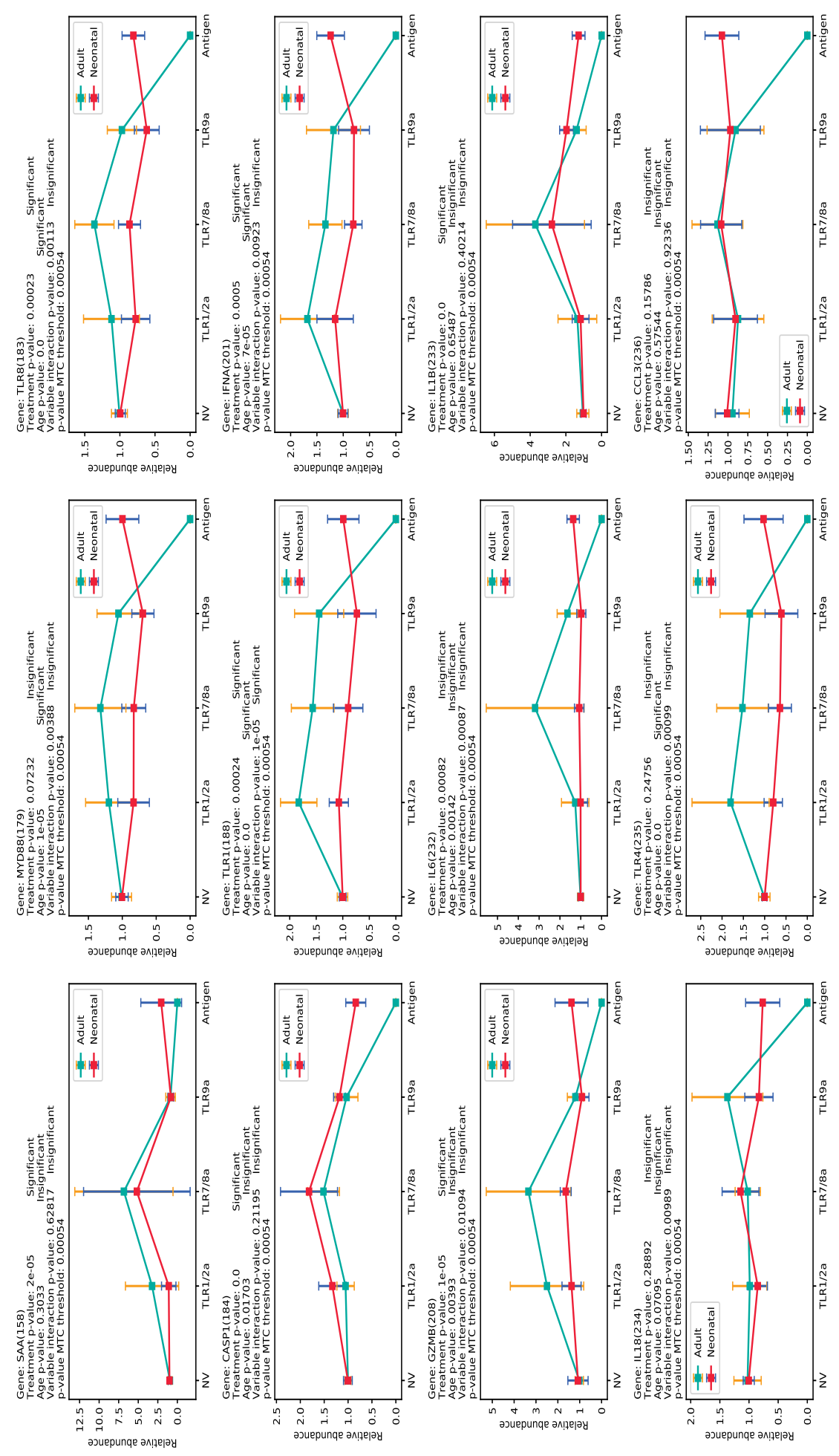

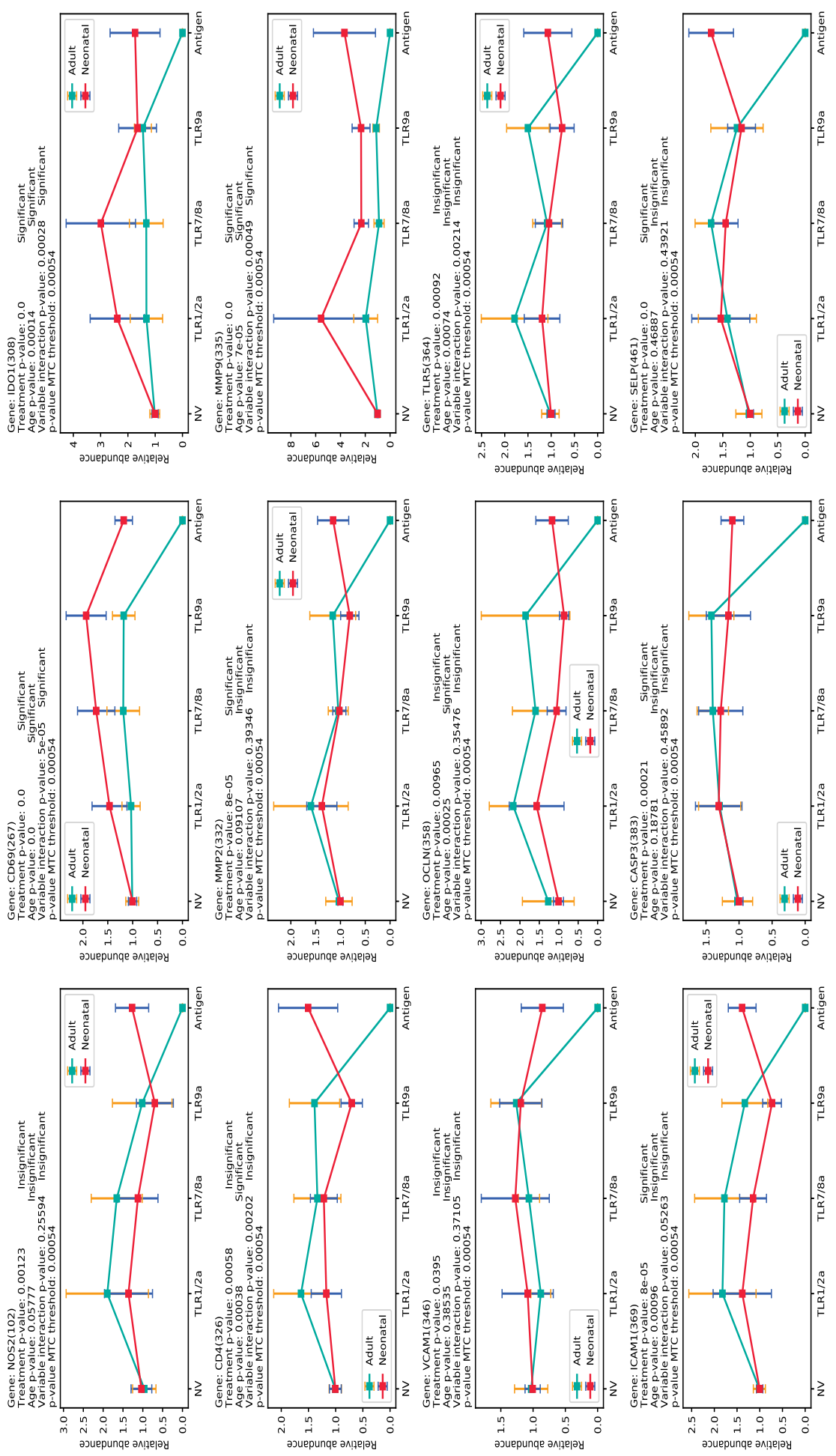

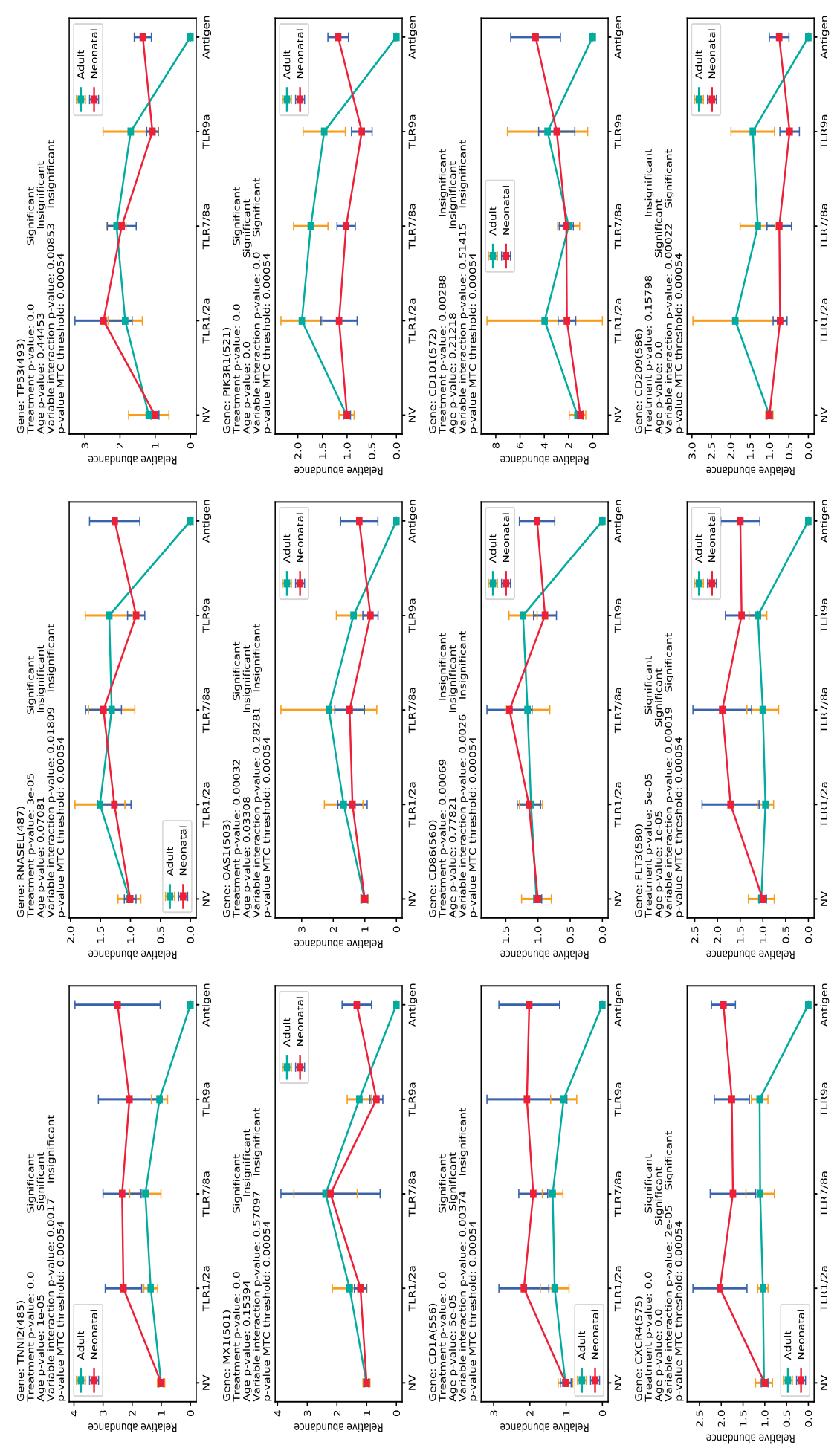

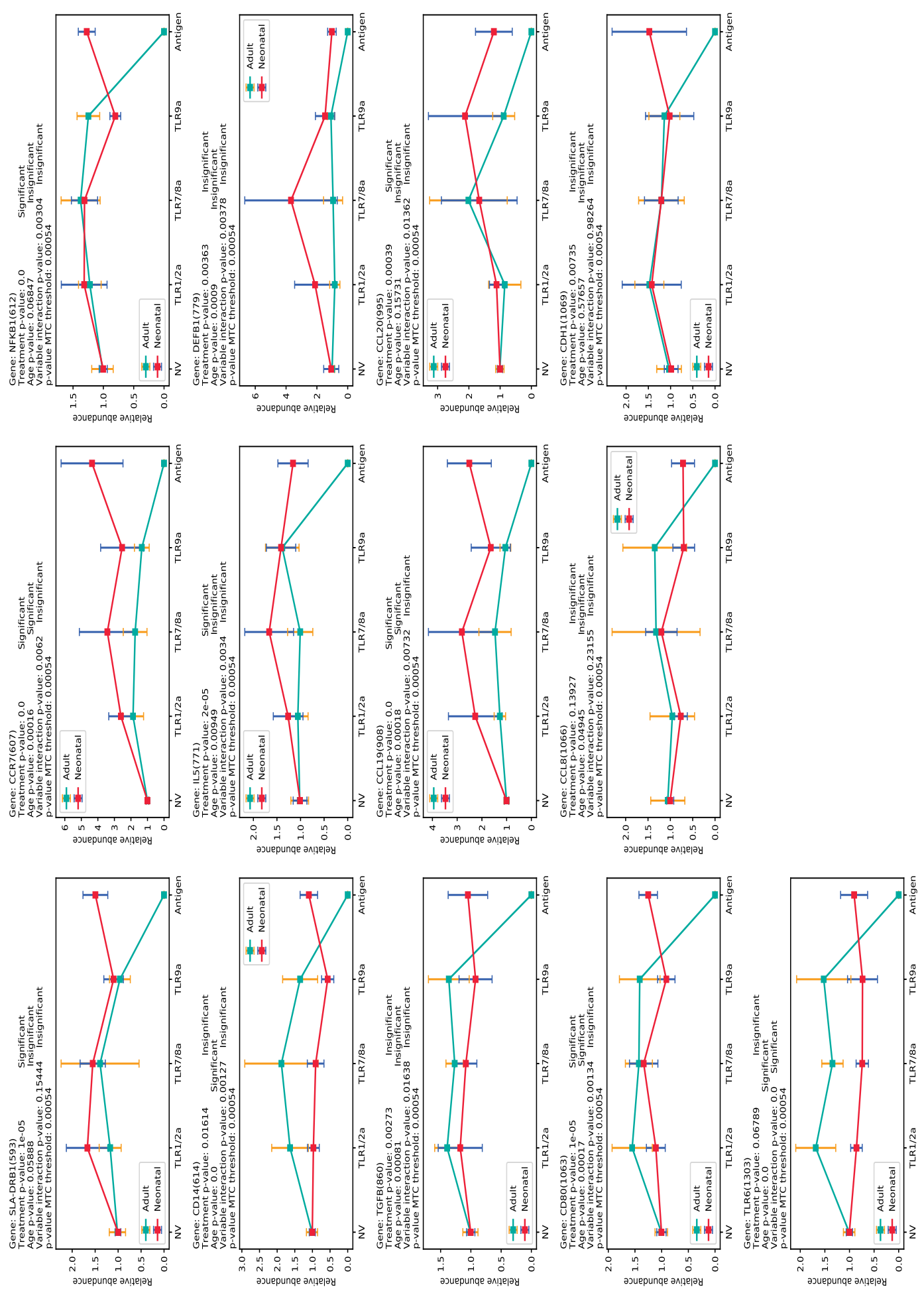


\section{References}

1 Heath, W. R. \& Carbone, F. R. The skin-resident and migratory immune system in steady state and memory: innate lymphocytes, dendritic cells and T cells. Nature immunology 14, 978-985, doi:10.1038/ni.2680 (2013).

2 Kabashima, K., Honda, T., Ginhoux, F. \& Egawa, G. The immunological anatomy of the skin. Nature reviews. Immunology 19, 19-30, doi:10.1038/s41577-018-0084-5 (2019).

3 Ferrari, L. et al. Lymphocyte activation as cytokine gene expression and secretion is related to the porcine reproductive and respiratory syndrome virus (PRRSV) isolate after in vitro homologous and heterologous recall of peripheral blood mononuclear cells (PBMC) from pigs vaccinated and exposed to natural infection. Veterinary immunology and immunopathology 151, 193-206, doi:10.1016/j.vetimm.2012.11.006 (2013).

4 Marshall, S., Sahm, L. J. \& Moore, A. C. The success of microneedle-mediated vaccine delivery into skin. Hum. Vaccin. Immunother., 0, doi:10.1080/21645515.2016.1171440 (2016).

5 Eble, P. L., Weerdmeester, K., van Hemert-Kluitenberg, F. \& Dekker, A. Intradermal vaccination of pigs against FMD with $1 / 10$ dose results in comparable vaccine efficacy as intramuscular vaccination with a full dose. Vaccine 27, 1272-1278, doi:10.1016/j.vaccine.2008.12.011 (2009).

6 Wang, Y. et al. Skin Vaccination against Rotavirus Using Microneedles: Proof of Concept in Gnotobiotic Piglets. PloS one 11, e0166038, doi:10.1371/journal.pone.0166038 (2016).

7 Summerfield, A., Meurens, F. \& Ricklin, M. E. The immunology of the porcine skin and its value as a model for human skin. Molecular immunology 66, 14-21, doi:10.1016/j.molimm.2014.10.023 (2015).

8 Pasparakis, M., Haase, I. \& Nestle, F. O. Mechanisms regulating skin immunity and inflammation. Nature reviews. Immunology 14, 289-301, doi:10.1038/nri3646 (2014).

9 Prausnitz, M. R. Engineering Microneedle Patches for Vaccination and Drug Delivery to Skin. Annu Rev Chem Biomol Eng 8, 177-200, doi:10.1146/annurev-chembioeng-060816-101514 (2017).

10 McCullough, K. C. \& Summerfield, A. Targeting the porcine immune system--particulate vaccines in the 21st century. Developmental and comparative immunology 33, 394-409, doi:10.1016/j.dci.2008.07.015 (2009).

11 Fehres, C. M., Garcia-Vallejo, J. J., Unger, W. W. \& van Kooyk, Y. Skin-resident antigen-presenting cells: instruction manual for vaccine development. Frontiers in immunology 4, 157, doi:10.3389/ fimmu.2013.00157 (2013).

12 Yazdi, A. S., Rocken, M. \& Ghoreschi, K. Cutaneous immunology: basics and new concepts. Seminars in immunopathology 38, 3-10, doi:10.1007/s00281-015-0545-x (2016).

13 Summerfield, A. \& McCullough, K. C. The porcine dendritic cell family. Developmental and comparative immunology 33, 299-309, doi:10.1016/j.dci.2008.05.005 (2009).

14 Banchereau, J. et al. Immunobiology of Dendritic Cells. Annu. Rev. Immunol. 18, 767-811, doi:10.1146/ annurev.immunol.18.1.767 (2000).

15 Marquet, F. et al. Characterization of dendritic cells subpopulations in skin and afferent lymph in the swine model. PloS one 6, e16320, doi:10.1371/journal.pone.0016320 (2011).

16 Marquet, F. et al. Pig skin includes dendritic cell subsets transcriptomically related to human CD1a and CD14 dendritic cells presenting different migrating behaviors and T cell activation capacities. Journal of immunology 193, 5883-5893, doi:10.4049/jimmunol.1303150 (2014). 
17 Buechler, C. et al. Regulation of scavenger receptor CD163 expression in human monocytes and macrophages by pro- and antiinflammatory stimuli. Journal of leukocyte biology 67, 97-103 (2000).

18 Holtkamp, D. J. et al. Assessment of the economic impact of porcine reproductive and respiratory syndrome virus on United States pork producers. Journal of Swine Health and Production 21, 72-84 (2013).

19 Nieuwenhuis, N., Duinhof, T. F. \& van Nes, A. Economic analysis of outbreaks of porcine reproductive and respiratory syndrome virus in nine sow herds. The Veterinary record 170, 225, doi:10.1136/vr.100101 (2012).

20 Nathues, H. et al. Cost of porcine reproductive and respiratory syndrome virus at individual farm level - An economic disease model. Preventive veterinary medicine 142, 16-29, doi:10.1016/j.prevetmed.2017.04.006 (2017).

21 Nan, Y. et al. Improved Vaccine against PRRSV: Current Progress and Future Perspective. Frontiers in microbiology 8, 1635, doi:10.3389/fmicb.2017.01635 (2017).

22 Renukaradhya, G. J., Meng, X. J., Calvert, J. G., Roof, M. \& Lager, K. M. Inactivated and subunit vaccines against porcine reproductive and respiratory syndrome: Current status and future direction. Vaccine 33, 3065-3072, doi:10.1016/j.vaccine.2015.04.102 (2015).

23 Jeong, J. et al. Vaccination with a porcine reproductive and respiratory syndrome virus vaccine at 1-dayold improved growth performance of piglets under field conditions. Veterinary microbiology 214, 113-124, doi:10.1016/j.vetmic.2017.12.023 (2018).

24 Saso, A. \& Kampmann, B. Vaccine responses in newborns. Seminars in immunopathology 39, 627-642, doi:10.1007/s00281-017-0654-9 (2017).

25 Siegrist, C. A. \& Aspinall, R. B-cell responses to vaccination at the extremes of age. Nature reviews. Immunology 9, 185-194, doi:10.1038/nri2508 (2009).

26 Mohr, E. \& Siegrist, C. A. Vaccination in early life: standing up to the challenges. Current opinion in immunology 41, 1-8, doi:10.1016/j.coi.2016.04.004 (2016).

27 Dewar, A. L., Doherty, K. V., Woods, G. M., Lyons, A. B. \& Muller, H. K. Acquisition of immune function during the development of the Langerhans cell network in neonatal mice. Immunology 103, 61-69, doi:10.1046/ j.1365-2567.2001.01221.x(2001).

28 Chang-Rodriguez, S., Ecker, R., Stingl, G. \& Elbe-Burger, A. Autocrine IL-10 partially prevents differentiation of neonatal dendritic epidermal leukocytes into Langerhans cells. Journal of leukocyte biology 76, 657-666, doi:10.1189/jlb.0204087 (2004).

29 Elbe-Burger, A. \& Schuster, C. Development of the prenatal cutaneous antigen-presenting cell network. Immunology and cell biology 88, 393-399, doi:10.1038/icb.2010.13 (2010).

30 Levy, O., Goriely, S. \& Kollmann, T. R. Immune response to vaccine adjuvants during the first year of life. Vaccine 31, 2500-2505, doi:10.1016/j.vaccine.2012.10.016 (2013).

31 de Brito, C. A., Goldoni, A. L. \& Sato, M. N. Immune adjuvants in early life: targeting the innate immune system to overcome impaired adaptive response. Immunotherapy 1, 883-895, doi:10.2217/imt.09.38 (2009). stimulation. Developmental and comparative immunology, doi:10.1016/j.dci.2018.03.012 (2018). 
33 Auray, G. et al. Characterization and Transcriptomic Analysis of Porcine Blood Conventional and Plasmacytoid Dendritic Cells Reveals Striking Species-Specific Differences. Journal of immunology 197, 4791-4806, doi:10.4049/jimmunol.1600672 (2016).

34 Auray, G. et al. Porcine neonatal blood dendritic cells, but not monocytes, are more responsive to TLRs stimulation than their adult counterparts. PloS one 8, e59629, doi:10.1371/journal.pone.0059629 (2013).

35 Magiri,R.B.etal.Responseofimmuneresponsegenestoadjuvantspoly[di(sodiumcarboxylatoethylphenoxy) phosphazene] (PCEP), CpG oligodeoxynucleotide and emulsigen at intradermal injection site in pigs. Veterinary immunology and immunopathology 175, 57-63, doi:10.1016/j.vetimm.2016.05.003 (2016).

36 McKay, P. F. et al. TLR4 and TLR7/8 Adjuvant Combinations Generate Different Vaccine Antigen-Specific Immune Outcomes in Minipigs when Administered via the ID or IN Routes. PloS one 11, e0148984, doi:10.1371/journal.pone.0148984 (2016).

37 van Aalst, S. et al. Routing dependent immune responses after experimental R848-adjuvated vaccination. Vaccine 36, 1405-1413, doi:10.1016/j.vaccine.2018.01.077 (2018).

38 Vreman, S. et al. Toll-like receptor agonists as adjuvants for inactivated porcine reproductive and respiratory syndrome virus (PRRSV) vaccine. Veterinary immunology and immunopathology 212, 27-37, doi:10.1016/j. vetimm.2019.04.008 (2019).

39 Shah, R. R. et al. The development of self-emulsifying oil-in-water emulsion adjuvant and an evaluation of the impact of droplet size on performance. Journal of pharmaceutical sciences 104, 1352-1361, doi:10.1002/ jps.24337 (2015).

40 Vrdoljak, A. et al. Induction of broad immunity by thermostabilised vaccines incorporated in dissolvable microneedles using novel fabrication methods. Journal of controlled release : official journal of the Controlled Release Society 225, 192-204, doi:10.1016/j.jconrel.2016.01.019 (2016).

41 Barington, K., Jensen, H. E. \& Skovgaard, K. Forensic aspects of gene expression signatures for age determination in bruises as evaluated in an experimental porcine model. Forensic Sci. Med. Pathol. 13, 151160, doi:10.1007/s12024-017-9869-2 (2017).

42 Pfaffl, M. W. A new mathematical model for relative quantification in real-time RT-PCR. Nucleic Acids Res. 29, e45, doi:10.1093/nar/29.9.e45 (2001).

43 Vandesompele, J. et al. Accurate normalization of real-time quantitative RT-PCR data by geometric averaging of multiple internal control genes. Genome Biol. 3, Research0034, doi:10.1186/gb-2002-3-7research0034 (2002).

44 Gauthier, B. E., Penard, L., Bordier, N. F., Briffaux, J. J. \& Ruty, B. M. Specificities of the Skin Morphology in Juvenile Minipigs. Toxicologic pathology 46, 821-834, doi:10.1177/0192623318804520 (2018).

45 Saitoh, A. \& Aizawa, Y. Intradermal vaccination for infants and children. Hum. Vaccin. Immunother. 12, 24472455, doi:10.1080/21645515.2016.1176652 (2016).

46 Drijkoningen, M., De Wolf-Peeters, C., Van der Steen, K., Moerman, P. \& Desmet, V. Epidermal Langerhans' cells and dermal dendritic cells in human fetal and neonatal skin: an immunohistochemical study. Pediatric dermatology 4, 11-17, doi:10.1111/j.1525-1470.1987.tb00745.x (1987).

47 Levy, O. Innate immunity of the newborn: basic mechanisms and clinical correlates. Nature reviews. Immunology 7, 379-390, doi:10.1038/nri2075 (2007). 
48 Kumar, S. K. \& Bhat, B. V. Distinct mechanisms of the newborn innate immunity. Immunol Lett 173, 42-54, doi:10.1016/j.imlet.2016.03.009 (2016).

49 de Jong, J. M. et al. Dendritic cells, but not macrophages or B cells, activate major histocompatibility complex class II-restricted CD4+ T cells upon immune-complex uptake in vivo. Immunology 119, 499-506, doi:10.1111/j.1365-2567.2006.02464.x (2006).

50 Oosterhoff, D. et al. Intradermal delivery of TLR agonists in a human explant skin model: preferential activation of migratory dendritic cells by polyribosinic-polyribocytidylic acid and peptidoglycans. Journal of immunology 190, 3338-3345, doi:10.4049/jimmunol.1200598 (2013).

51 Schneider, L. P. et al. Intradermally administered TLR4 agonist GLA-SE enhances the capacity of human skin DCs to activate T cells and promotes emigration of Langerhans cells. Vaccine 30, 4216-4224, doi:10.1016/j. vaccine.2012.04.051 (2012).

52 Caproni, E. et al. MF59 and Pam3CSK4 boost adaptive responses to influenza subunit vaccine through an IFN type I-independent mechanism of action. Journal of immunology 188, 3088-3098, doi:10.4049/ jimmunol.1101764 (2012).

53 Zhang, W. W. \& Matlashewski, G. Immunization with a Toll-like receptor 7 and/or 8 agonist vaccine adjuvant increases protective immunity against Leishmania major in BALB/c mice. Infect. Immun. 76, 3777-3783, doi:10.1128/iai.01527-07 (2008).

54 Neeland, M. R. et al. Transcriptional profile in afferent lymph cells following vaccination with liposomes incorporating CpG. Immunology 144, 518-529, doi:10.1111/imm.12401 (2015).

55 Ahlberg, V., Lovgren Bengtsson, K., Wallgren, P. \& Fossum, C. Global transcriptional response to ISCOMMatrix adjuvant at the site of administration and in the draining lymph node early after intramuscular injection in pigs. Developmental and comparative immunology 38, 17-26, doi:10.1016/j.dci.2012.03.005 (2012).

56 Fossum, C. et al. Early inflammatory response to the saponin adjuvant Matrix-M in the pig. Veterinary immunology and immunopathology 158, 53-61, doi:10.1016/j.vetimm.2013.07.007 (2014).

57 Magiri, R., Lai, K., Huang, Y., Mutwiri, G. \& Wilson, H. L. Innate immune response profiles in pigs injected with vaccine adjuvants polydi(sodium carboxylatoethylphenoxy)phosphazene (PCEP) and Emulsigen. Veterinary immunology and immunopathology 209, 7-16, doi:10.1016/j.vetimm.2019.02.004 (2019).

58 Holbrook, B. C.et al. A Novel R848-Conjugated Inactivated Influenza Virus Vaccine Is Efficacious and Safe in a Neonate Nonhuman Primate Model. Journal of immunology 197, 555-564, doi:10.4049/jimmunol.1600497 (2016).

59 Vono, M. et al. Overcoming the Neonatal Limitations of Inducing Germinal Centers through LiposomeBased Adjuvants Including C-Type Lectin Agonists Trehalose Dibehenate or Curdlan. Frontiers in immunology 9, 381, doi:10.3389/fimmu.2018.00381 (2018).

60 Basha, S., Surendran, N. \& Pichichero, M. Immune responses in neonates. Expert Rev. Clin. Immunol. 10, 1171-1184, doi:10.1586/1744666x.2014.942288 (2014).

61 Le Luduec, J. B. et al. Intradermal vaccination with un-adjuvanted sub-unit vaccines triggers skin innate immunity and confers protective respiratory immunity in domestic swine. Vaccine 34, 914-922, doi:10.1016/j.vaccine.2015.12.058 (2016). 


\title{
Toll-Like receptor agonists as adjuvants for inactivated porcine reproductive and respiratory syndrome virus (PRRSV) vaccine
}

\author{
Sandra Vreman ${ }^{1,2}$, Joanne McCaffrey ${ }^{3}$, Ditta J. Popma-de Graaf ${ }^{1}$, \\ Hans Nauwynck ${ }^{4}$, Huub F.J. Savelkoul ${ }^{2}$, Anne Moore ${ }^{5}$, Johanna M.J. Rebel ${ }^{6}$, \\ Norbert Stockhofe-Zurwieden ${ }^{1}$
}

\footnotetext{
${ }^{1}$ Wageningen Bioveterinary Research, Wageningen University \& Research, The Netherlands

${ }^{2}$ Cell Biology \& Immunology group, Wageningen University \& Research, The Netherlands

${ }^{3}$ Xeolas, Pharmaceuticals, Dublin Ireland

${ }^{4}$ Laboratory of Virology, Faculty of Veterinary Medicine, Ghent University, Belgium

${ }^{5}$ School of Pharmacy, School of Biochemistry and Cell Biology, University College Cork, Cork, Ireland.

${ }^{6}$ Wageningen Livestock Research, Wageningen University \& Research, The Netherlands
} 


\begin{abstract}
Toll-like receptor (TLR) agonists can effectively stimulate antigen-presenting cells (APCs) and are anticipated to be promising adjuvants in combination with inactivated vaccines. In this study, the adjuvant potential of three different TLR-agonists were compared with an oil-inwater (O/W) adjuvant in combination with inactivated porcine reproductive and respiratory syndrome virus (iPRRSV) applied by different administration routes: intramuscular (i.m.) or into the skin using dissolving microneedle (DMN) patches. Pigs received a prime vaccination followed by a booster vaccination four weeks later. TLR1/2 (Pam3Cys), TLR7/8 (R848) or TLR9 (CpG ODN) agonists were used as adjuvant in combination with iPRRSV strain 07V063. O/W adjuvant (Montanide ${ }^{\mathrm{TM}}$ ) was used as reference control adjuvant and one group received a placebo vaccination containing diluent only. All animals received a homologous challenge with PRRSV three weeks after the booster vaccination. Antibody and IFN- $\gamma$ production, serum cytokines and viremia were measured at several time-points after vaccination and/or challenge, and lung pathology at necropsy. Our results indicate that a TLR $1 / 2,7 / 8$ or 9 agonist as adjuvant with iPRRSV does not induce a detectable PRRSV-specific immune response, independent of the administration route. However, the i.m. TLR9 agonist group showed reduction of viremia upon challenge compared to the non-vaccinated animals, supported by a non-antigen-specific IFN- $\gamma$ level after booster vaccination and an anamnestic antibody response after challenge. Montanide ${ }^{\mathrm{TM}}$-adjuvanted iPRRSV induced antigen-specific immunity after booster combined with reduction of vireamia. Skin application of TLR7/8 agonist, but not the other agonists, induced a local skin reaction. Further research is needed to explore the potential of TLR agonists as adjuvants for inactivated porcine vaccines with a preference for TLR9 agonists.
\end{abstract}




\section{Introduction}

Porcine reproductive and respiratory syndrome (PRRS) is an important viral disease affecting swine health and welfare. The swine industry reports large economic losses due to the PRRS virus (PRRSV) ${ }^{1,2}$ and vaccination is essential to prevent PRRSV infection and transmission. The disease is widespread globally, and after infection there is a variable morbidity and mortality with reproductive disorders in sows and respiratory symptoms often with co-infections in growing and finishing pigs.

PRRSV is an enveloped, positive-stranded RNA virus of the Arteriviridae family of which two distinct viral species are known ${ }^{3}$ : PRRSV-1, most relevant in Europe and divided in three subtype ${ }^{4}$ with a different pathogenicity, and PRRSV-2, mostly occurring in North America and South East Asia ${ }^{5,6}$. PRRSV- 1 and PRRSV-2 are constantly evolving and new strains appear, which complicates the development of a protective vaccine ${ }^{7}$. Several commercial vaccines have been licenced for the different types of PRRSV ${ }^{8}$. In general, modified-live-virus vaccines induce a good protection against homologous infections, however cross protection and safety are points of concern ${ }^{9}$. Conversely, inactivated PRRSV (iPRRSV) vaccines are safe to use, but provide limited protection ${ }^{9-12}$. Safe and effective iPRRSV vaccines are needed for virus control and adjuvants such as Toll-like receptor (TLR) agonists could help to increase the vaccine efficacy.

TLRs are a family of conserved pattern recognition receptors (PRRs) that recognize specific parts of microbial pathogens and activation of these receptors links the innate and adaptive immune response by activation of APCs ${ }^{13}$. Vaccines can benefit from this property by using specific synthetic TLR agonists (TLRa) as adjuvant to increase the magnitude and the efficacy of the immune response ${ }^{14}$. TLRa have been extensively investigated in mice and humans for their immunostimulatory properties ${ }^{15,16}$ and their applications as adjuvants. However, functional differences of specific TLRs and variation between species ${ }^{17,18}$ necessitates investigation of the efficacy of individual TLRa in porcine vaccines. in vitro stimulation of various porcine TLRs demonstrated that predominantly TLR2a, 7a and 9a ${ }^{19,20}$ induced activation of APCs, which is important for antigen uptake and transport to the draining lymph nodes. Also in vivo, individual administration of TLR2a ${ }^{21}, 7 \mathrm{a}$ or $9 a^{16,22,23}$ enhanced the quality and quantity of the host adaptive immune response in several vaccine studies in humans and mice. Therefore, TLR2a, 7a and 9a are interesting candidates as adjuvants for iPRRSV vaccine development.

The search for an optimal antigen-adjuvant combination is a critical step in the development of inactivated vaccines ${ }^{24}$. TLRa have been previously studied in combination with PRRSV-2. CL097, a TLR7a, combined with iPRRSV-2 enhanced the immune response and protection against PRRSV in pigs ${ }^{25}$, and the effect could be mimicked in mice by using the TLR7a SZU101 combined with PRRSV- ${ }^{26}$. Other porcine studies have shown that iPRRSV-2 in combination with a TLR9a (CpG ODN) enhanced protection after challenge with a highly pathogenic PRRSV-2 strain ${ }^{27,28}$. To our knowledge, TLR2a have not been used in recent PRRSV vaccination research. In this study we focussed on PRRSV-1, which is most 
common in Western Europe and is known to have large genomic differences compared to PRRSV-2 ${ }^{29}$.

Additionally to the use of adjuvants, the route of administration can be crucial to achieve optimal protection after vaccination. Different delivery routes with the same vaccine can induce variation in immune response and efficacy ${ }^{30,31}$. The efficacy of TLR9a CpG ODN with live-attenuated PRRSV vaccine was route-dependent: i.m. administration reduced the viral shedding more than the intranasal route ${ }^{32}$. The magnitude of the immune response and the cytokine profile were differentially affected using intradermal or intranasal vaccination routes in a mouse model with a TLR7/8a (R848) as adjuvant 33. Dissolving microneedle (DMN) patches deliver the vaccine directly into the skin and possibly thereby target high numbers of APCs that are constitutively present in the skin. Skin-based vaccination may thus result in equivalent or stronger immune responses compared to traditional i.m or subcutaneous routes ${ }^{34,35}$, especially when combined with appropriate adjuvants ${ }^{36}$. Other reported advantages of skin vaccination are dose reduction ${ }^{37}$ and generation of mucosal immune responses, which is especially important for respiratory diseases ${ }^{38,39}$. Previous studies with TLRa showed that intradermal injection of TLR9a in pigs may promote recruitment of innate immune cells and Th1 cytokine production ${ }^{40}$, while a TLR7a can augment antigen-specific serum responses in mini-pigs ${ }^{41}$. Overall, the skin seems a promising administration site to combine with TLRa in iPRRSV vaccines. However, to our knowledge, no studies to date have evaluated the microneedlebased skin delivery of TLRa to non-mouse species ${ }^{42}$.

In this study we investigated the immunogenicity and efficacy of TLR1/2a (Pam3Cys), TLR7/8a (R848) or TLR9a (CpG ODN) as adjuvant with iPRRSV-1 administered by i.m. injection or skin-based DMN patches. The antigen-specific PRRSV antibody response, IFN- $\gamma$ production, reduction of viremia, serum cytokines and lung pathology after challenge were assessed to define the immune responses of the pigs.

\section{Material and methods}

\section{Virus}

PRRSV strain Belgium A (07V063) is a low pathogenic subtype 1 PRRSV-1 isolate. This strain was isolated from an aborted foetus from a Belgian farm, by inoculation of porcine alveolar macrophages (PAM) ${ }^{43}$. This strain was used in recent studies describing viral, clinical and pathological data ${ }^{44,45}$ and as inactivated virus for vaccination ${ }^{11}$.

For challenge, MARC-Sn grown stocks were prepared of the isolates 07V063 (4th passage on MARC-Sn cells). For vaccine preparation, MARC-Sn cell culture supernatants of 07V063 (2nd passage on PAM + 2 passages on MARC-Sn), were purified via ultracentrifugation as described previously ${ }^{12}$. 


\section{Virus inactivation and quality control}

Purified virus (07V063) was suspended in RPMI 1640 (Invitrogen) to a titer of $10^{9.7} \mathrm{TCID}_{50} /$ $\mathrm{mL}$. Subsequently, the virus was inactivated using binary ethylenimine (BEI) as described before ${ }^{12}$, and inactivated virus was stored at $-70^{\circ} \mathrm{C}$. To confirm that all virus was completely inactivated, a complete vaccine dose of 07V063 was inoculated on MARC-145 cells and subsequently passaged twice. As a positive control, MARC-145 cells were inoculated with $1 \mathrm{~mL}$ of non-inactivated 07V063. The MARC-145 cells were routinely checked for cytopathic effect (CPE) and ultimately stained for the PRRSV nucleocapsid protein via an immunoperoxidase monolayer assay (IPMA) using monoclonal antibody $13 \mathrm{E} 2{ }^{46}$. No CPE or positive nucleocapsid staining was detected in cells that were inoculated with inactivated virus, while clear CPE and nucleocapsid staining were observed in cell cultures that were inoculated with non-inactivated virus. Since conservation of entry of inactivated virus may serve as a quality control for the preservation of antigenic properties, the effect of $\mathrm{BEl}$ inactivation on virus attachment and internalization into macrophages was examined as described previously ${ }^{12}$. Non-inactivated virus suspensions were included as positive controls. The entry experiment showed that the binding and internalization kinetics of all BEl-inactivated virus stocks are similar to those observed for the non-inactivated virus stocks.

\section{Vaccines}

All the vaccines (i.m. and DMN-patches) contained the same dose of BEl-inactivated PRRSV 07V063 $\left(1.0 \times 10^{8.0} \mathrm{TCID}_{50}\right)$ with different adjuvants. Montanide ${ }^{\mathrm{m}}$ ISA 28 R VG (kindly provided by SEPPIC), an oil-in-water (O/W) emulsion compromising a blend of a mineral and non-mineral oil, was selected as reference control adjuvant, as a previous study with PRRSV 07V063 ${ }^{11}$ showed a clear antibody response with a similar O/W adjuvant. Montanide ${ }^{\mathrm{mm}}$ was administered only i.m., as $15 \%$ volume per volume (v/v) in a final volume of $1 \mathrm{ml}$ according to manufacturer's instruction. The adjuvants of the experimental groups were composed of different TLRa: TLR1/2a; Pam3Cys L2000 from EMC micro-collections, TLR7/8a; R848, Resiquimod from InvivoGen or TLR9a; CpG ODN-type A sequence D32, 5'-ggTGCGTCGACGCAGggggg-3', from Eurofins Genomics 19,20. All vaccines (i.m. and DMN-patches) contained $250 \mu \mathrm{g}$ of the individual TLRa (Figure 1b). This adjuvant dose was based on study results, which were obtained in different animal species and using different delivery routes, for Pam3Cys ${ }^{47}, \mathrm{R} 8488^{41,48}, \mathrm{CpG}$ ODN ${ }^{27,49}$ and the maximum dose which could be incorporated in the DMN- patches. The i.m. vaccines were freshly prepared on the day of vaccination and mixed with PBS to a volume of $1 \mathrm{ml}$.

Each DMN patch contained 225 microneedles, $500 \mu \mathrm{m}$ in length, in a $9 \mathrm{~cm}^{2}$ area. They were prepared as previously described ${ }^{31}$, using trehalose and polyvinyl alcohol (PVA) as excipients. The iPRRSV and TLRa was dispersed homogenously throughout the full volume of the microneedle. Briefly, formulation was delivered directly onto the waterfilled microneedle cavities in a polydimethylsiloxane (PDMS) mould at a rate of 1-3 $\mu \mathrm{L}$ / 
min. Formulation in the moulds was dried overnight at room temperature and then pulled from the mould onto medical grade adhesive tape (1525 I Poly Med tape, $3 \mathrm{M}$ ). The patches were immediately packaged and sealed in moisture-barrier bags containing desiccant and stored at $2-8^{\circ} \mathrm{C}$ prior to shipping and administration.

\section{Animals and housing}

Forty-eight ( $\mathrm{n}=48$ ) male six-week-old pigs (Topigs Norsvin Z-line, commercial breed) were purchased from a PRRSV-negative, high health status farm (van Beek SPF Varkens B.V.) in the Netherlands. The seronegative-PRRSV status of the pigs was confirmed upon arrival at the research facility with a commercial antibody ELISA. After arrival, the pigs were stratified based on their weight and family background followed by a randomisation to eight groups ( $n=6$ for each group). Before challenge the animals were housed in eight separated boxes in one stable. One week before challenge the animals were moved to 8 separate rooms in an isolation unit with HEPA-filtered air. All the stables were enriched with straw and toys. Standard feed for finishing pigs was provided twice a day and the pigs had unlimited access to water. The experiment started after one week of acclimatization. The animal experiment was conducted in accordance with the Dutch animal experimental and ethical requirements and the project license application was approved by the Dutch Central Authority for Scientific Procedures on Animals (CCD) (Permit number: ADV401002015356).

\section{Experimental design}

All the pigs except for the non-vaccinated (NV) group received a prime vaccination (D0) with iPRRSV and different adjuvants (Fig. $1 \mathrm{~A}$ and $\mathrm{B}$ ) at 7 weeks of age followed by a booster vaccination (D28) at 11 weeks of age. The NV control group received $1.0 \mathrm{ml}$ of PBS i.m. in the right hind leg. The reference control group (O/W con) group received $\mathrm{O} / \mathrm{W}$ adjuvant Montanide i.m. $(1.0 \mathrm{ml})$ in the right hind leg. The six experimental groups with the different TLR agonists were divided in three i.m groups (imTLR1/2a, 7/8a and 9a) and three groups with DMN-patches (skiTLR1/2a, 7/8a and 9a).

The lateral right hind leg was used for the i.m. injection $(1.0 \mathrm{ml})$ and the DMN-patch was applied at the medial side of the left hind leg. Before vaccination with a DMN-patch the pigs were sedated i.m. with a mixture of Zoletil ${ }^{\circ}\left(4 \mathrm{mg} / \mathrm{kg}\right.$, Virbac) and Sedamun ${ }^{\circ}(2 \mathrm{mg} /$ $\mathrm{kg}$, Dechra) to ensure correct and equal application and by this optimize standardisation of skin vaccination. Pigs receiving an i.m vaccination were not sedated. The DMN-patches were removed after $24 \mathrm{~h}$. Three weeks after the booster vaccination (D49; 14 weeks of age) all animals were challenged intranasally with PRRSV 07 V063 $\left(10^{6} \mathrm{TCID}_{50}\right)$ in PBS $(1.0 \mathrm{ml}$ per nostril). Three weeks post challenge (D70) the animals were euthanized with pentobarbital $\left(150 \mathrm{mg} / \mathrm{kg}\right.$ intravenous) after i.m. sedation with a mixture of Zoletil ${ }^{\circ}$ ( $4 \mathrm{mg} / \mathrm{kg}$, Virbac) and Sedamun ( $2 \mathrm{mg} / \mathrm{kg}$, Dechra), followed by exsanguination. During necropsy different lung lobes were collected for pathological examination. 
A Prin

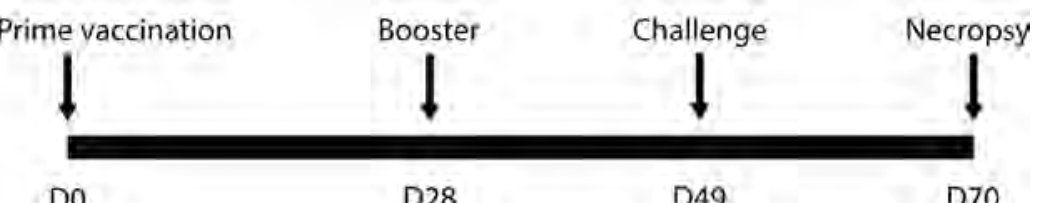

Do

D28

D49

D70

B

\begin{tabular}{|c|c|c|c|c|c|c|c|c|}
\hline Group & $\begin{array}{c}1 \\
n=6\end{array}$ & $\begin{array}{c}2 \\
n=6\end{array}$ & $\begin{array}{c}3 \\
n=6\end{array}$ & $\begin{array}{c}4 \\
n=6\end{array}$ & $\begin{array}{c}5 \\
n=6\end{array}$ & $\begin{array}{c}6 \\
n=6\end{array}$ & $\begin{array}{c}7 \\
n=6\end{array}$ & $\begin{array}{c}8 \\
n=6\end{array}$ \\
\hline Name & NV & $\begin{array}{l}\text { Orw-con } \\
\mathrm{im}\end{array}$ & skiTLRJ/2a & skiTLR7/Ba & skiTLR9a & IIITLR1/2a & inTLR7/8a & imTLR9a \\
\hline Adjuvant & - & $\begin{array}{l}150 \mu l(15 \%) \\
\text { Montanide }\end{array}$ & $\begin{array}{l}250 \mu g \\
\text { Pam3Cys }\end{array}$ & $\begin{array}{c}250 \mu g \\
R 848\end{array}$ & $\begin{array}{c}250 \mu g \\
\mathrm{CpG}\end{array}$ & $\begin{array}{l}250 \mu \mathrm{g} \\
\text { Pam3Cys }\end{array}$ & $\begin{array}{c}250 \mu \mathrm{gg} \\
\mathrm{R} 848\end{array}$ & $\begin{array}{c}250 \mu 9 \\
\mathrm{CpG}\end{array}$ \\
\hline
\end{tabular}

Figure 1: Experimental design and group overview.

(A) Study design with prime vaccination at D0, booster vaccination at D28 followed by a homologous challenge at D49 and necropsy at D70; (B) Group overview: non-vaccinated animals (NV); in all vaccinated groups iPRRSV 07V063 antigen was used either with Montanide ${ }^{\mathrm{TM}}$ ISA 28R VG (O/W con) or Toll-like receptor agonist (TLR1/2a, TLR7/8a or TLR9a); im, indicates groups with i.m. vaccination; ski indicates groups with skin vaccination with dissolving microneedle (DMN)-patch.

\section{Monitoring of post-vaccination and challenge reaction}

After vaccination the injection site or DMN-patch application area were monitored over 4 days for local effects, such as redness and swelling of skin, graded from 0 (no changes) to 3 (clear redness and swelling of skin). Rectal temperature and clinical signs were recorded daily from two days before vaccination until 4 days after vaccination, and twice a day from one week before challenge until the end of the experiment respectively. Fever was defined as body temperature higher than $40^{\circ} \mathrm{C}$. Pigs were monitored twice a day for PRRSV-related clinical signs: liveliness, coughing, breathing, skin changes ears and appetite, as previously described ${ }^{45}$. Pigs were weighed upon arrival (D-7), three weeks after booster vaccination (D48) and during the challenge (D56, D63 and D70).

\section{Blood sample collection and pre-treatment}

Serum samples were collected at D-4, D21, D28, D35, D42, D48, D52, D54, D57, D59, D63 and D66 to determine virus titres and antibody levels. Serum samples from D52 (3 days after challenge) were used for cytokine determination. The samples were stored directly at $-80^{\circ} \mathrm{C}$ until analysis.

Heparin stabilized blood samples (approximately $15 \mathrm{ml}$ ) were collected at D21 and D42 for isolation of PBMCs for IFN- $\gamma$ ELISpot assay. Collected blood was diluted 1:1 with PBS within $2 \mathrm{~h}$ of collection and transferred to a Leucosep tube using a $60 \%$ FICOLLPAQUE ${ }^{\mathrm{TM}}$ Plus density- gradient to isolate the PBMCs. Remaining red blood cells were lysed with ACK lysis buffer. PBMC were rested overnight at $4^{\circ} \mathrm{C}$ before further analysis or 
freezing. Additionally, on D-4 we collected whole blood samples in Paxgene RNA tubes for future analysis on genetic markers.

\section{PRRSV serology}

Serum samples were thawed on ice and the PRRSV-specific IgG antibodies in serum samples were tested with an indirect antibody ELISA (IDEXX PRRS X3 enzyme immunoassay) designed to detect PRRSV IgG antibodies using a recombinant nucleocapsid $(\mathrm{N})$ protein as the coating antigen and an anti-pig immunoglobulin (lg)G-HRP conjugate in a second step ${ }^{50}$. The kit was used according to the manufacturer's instruction and a sample-topositive ratio of equal or greater than 0.4 was considered positive. Samples that tested negative were consequently given a numerical value of 0.00 .

\section{IFN- $\gamma$ ELISpot assay}

The number of antigen-specific IFN- $\gamma$ secreting cells (SCs) was determined with an enzyme-linked immunospot assay) ELISpot assay kit (Porcine IFN- $\gamma$ ELISpot ${ }^{\text {PLUS }}$ (ALP) from Mabtech) according to the manufacturer's instruction. Briefly, $0.5 \times 10^{6} \mathrm{PBMC}$ were added to antibody pre-coated 96 well plates in RPMI 1640 medium (Gibco ${ }^{\circ}$ ) supplemented with $10 \% \mathrm{FBS}$ and $1 \%$ Penicillin-Streptomycin (Gibco ${ }^{\circ}$. Cells were stimulated with PRRSV 07 V063 with a multiplicity of infection (MOI) of 0.1 based on previous ELISpot results with PRSSV-1 stimulation ${ }^{45,51,52}$. Cells without stimulus (medium only) were used as negative control. ConA ( $3 \mu \mathrm{g} / \mathrm{ml}$ from Sigma-Aldrich) stimulated cultures were used as positive control. All samples were analysed in triplicate. After incubation at $37^{\circ} \mathrm{C}$ and $5 \% \mathrm{CO}_{2}$ for $24 \mathrm{~h}, \mathrm{PBMCs}$ were discarded and secondary IFN- $\gamma$ antibody and streptavidin was added according to manufacturer's instruction. Spot development was stopped by washing plates under tap water. The number of specific IFN- $\gamma \mathrm{SC}$, as determined using an Immunospot ${ }^{\circ} \mathrm{S} 4$ Analyzer (Cellular Technology Ltd.), were calculated as the average number of spots in the triplicate PBMC cultures stimulated with virus minus the average number of spots in the triplicate PBMC cultures exposed to culture medium only (non-specific response or background).

\section{Cytokine production measured by multiplex assay}

Protein concentrations of IFN- $a$, TNF, IL- 4 and IL- 6 were measured in the serum at 3 days after challenge. A custom-designed multiplex Cytometric Bead Array (PorcineProcartaplex; eBioscience) was used according to the manufacturer's instructions and read on a Luminex machine (Luminex $200^{\mathrm{TM}}$ ). Cytokine concentrations were determined using $\mathrm{XPONENT}^{\circ}$ software. The detection limits of the cytokines were $0.72 \mathrm{pg} / \mathrm{ml}$ (IFN- $\mathrm{a}), 7.57 \mathrm{pg} / \mathrm{ml}$ (TNF), $1.55 \mathrm{pg} / \mathrm{ml}$ (IL-4) and $6.32 \mathrm{pg} / \mathrm{ml}$ (IL-6), respectively.

\section{PRRSV titers in serum measured by virus titration}

Virus titers in serum were determined by virus titration on PAM, obtained from 3 to 5 week old piglets from a PRRSV and PCV2 -free herd in the Netherlands. The PAM were cultured in 24-well plates (Greiner) at a concentration of $5 \times 10^{5}$ cells/well in $1 \mathrm{ml}$ RPMI 
1640 medium $\left(\mathrm{Gibco}^{\circledast}\right.$ ) supplemented with 10\% FBS and 1\% Penicillin-Streptomycin $\left(\mathrm{Gibco}^{\circ}\right)$. 10-fold dilution series of the serum samples (four dilutions for each sample) were inoculated to the PAM (250 ul) and plates were incubated at $37^{\circ} \mathrm{C}$ in a $5 \% \mathrm{CO}_{2}$ humidified atmosphere. After 3 days, the monolayers were washed in $0.15 \mathrm{M} \mathrm{NaCl}$ solution, dried and frozen. The monolayers were stained by IPMA to visualize infection in the cells ${ }^{53}$ using a 1:500 dilution of the monoclonal antibody SDOW17-A (Rural Technologies) against the nucleocapsid protein of PRRSV. Virus titers were calculated as $\operatorname{TCID}_{50}$ as described by ${ }^{54}$. The detection limit of the virus titration was $1.0 \log _{10} \mathrm{TCID}_{50} / \mathrm{ml}$. To check the sensitivity of the PAM, all cell batches were assessed in the virus titrations with a PRRSV stock (07V063) with a known virus titer.

\section{Pathology}

A complete necropsy was performed on all pigs. An ECVP board-certified veterinary pathologist (SV) performed the macroscopic examination of the lung lesions. Macroscopic (or gross) lung lesions were scored to estimate the percentage of affected lung tissue by pneumonia according to ${ }^{55}$. For histology, samples were taken from three predefined locations of the right lung: anterior lobe, middle lobe and caudal lobe. Tissues were fixed in $4 \%$ neutral-buffered formaldehyde and routinely processed and embedded in paraffin. Consecutive 4 ųm thick sections were cut and stained with hematoxylin and eosin (HE).

HE stained slides were analysed semi-quantitively in a "blinded" manner by the same veterinary pathologist. The lung histology was scored according to ${ }^{56}$ with slight modifications. Briefly, the three lung sections of the right lobe were scored for the presence of a perivascular and peribronchiolar inflammatory infiltrate from 0 (no findings) to 5 (extended manifestation) and for the alveolar wall infiltrate (0-5). To compare the histological findings between the groups, the perivascular and peribronchiolar scores of the three sections per lung were added to an overall score, which could add up to a maximum of 30 .

\section{Statistical analysis}

Data were analysed using SPSS Statistics version 23. The distribution of the data was explored with the descriptive statistics. Data with an overall normal distribution (ELISA and virus titration; Fig. 3 and 6 respectively) were analysed with a one-way ANOVA followed by a post-hoc Dunnett test (2-sided) using non-vaccinated animals and O/W control group as reference groups. Non-parametric data (skin changes, ELISpot, cytokines, an lung pathology; Fig. 2, 4, 5 and 7 respectively) were analysed with a Kruskal-Wallis test followed by a post-hoc Mann-Whitney $U$ test for multiple comparisons. Association analysis was performed with a Pearson correlation test (2-tailed) . P-values less than 0.05 were considered statistical significant $\left({ }^{*} p<0.05\right)$. 


\section{Results}

\section{Vaccine safety and challenge reaction}

None of the vaccines induced any systemic adverse effects, such as raise in body temperature, loss of appetite and activity or reduced weight gain (data not shown). However, after application of TLRa and iPRRSV in DMN patches, a transient local reaction was observed in some animals, characterized by a variable local redness and swelling of the skin graded from 0 (no change) to 3 (swelling and redness of the skin) (Fig. 2A). The skiTLR7/8a group showed significantly more skin reactions than skiTLR1/2a or 9a group (Fig. 2B and C) up to 2 days after primary and 1 day after the booster immunization. There was no difference in severity between the prime and booster vaccination. All of the local skin reactions disappeared within 4 days post vaccination. No local reaction was observed after i.m. administration.

Following challenge, none of the pigs in any of the study groups exhibited PRRSVrelated clinical signs or raise in body temperature. Additionally, there was no significant difference in weight or weight gain between the groups (results not shown).
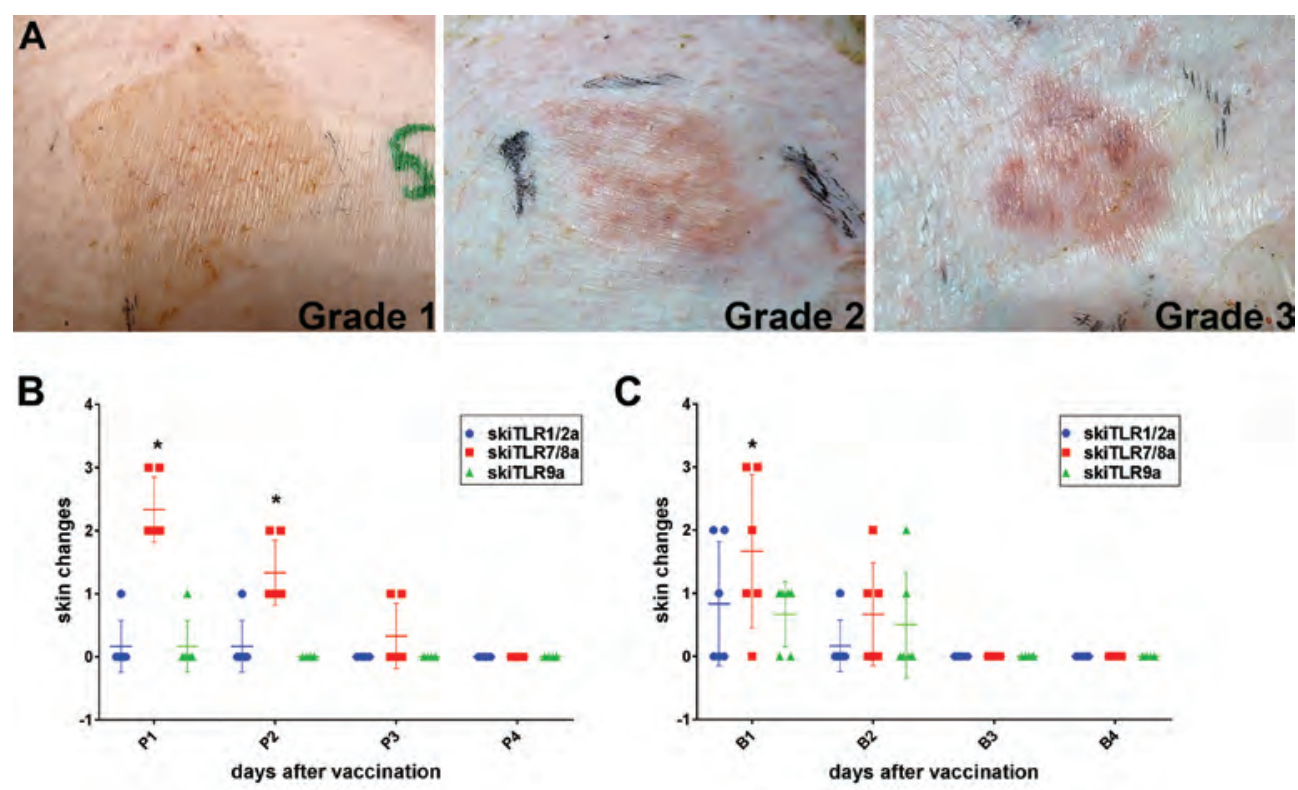

Figure 2: Skin reaction after dissolving microneedle (DMN) patch application with different Toll-like receptor (TLR) agonists.

(A) Examples of grading of skin changes (grade 1-3) based on redness and swelling of skin; normal skin (grade 0 ) is visible around the skin changes (B) Skin reaction overview from day 1 till day 4 after prime vaccination $(P)$ and after booster vaccination $(C)$. Each bar represents the median of a group of 6 animals with the $95 \% \mathrm{Cl}$. ( ${ }^{*} p<0.05$ compared to other groups). 


\section{PRRSV specific IgG antibody response}

PRRSV-specific IgG antibodies were measured in the serum to determine the humoral immune response. After booster vaccination (D35) only the O/W control group showed a detectable PRRSV antibody response (in 4 of the 6 animals) (Fig. 3A and B), while none of the animals in the experimental TLRa groups were able to develop a specific antibody response after booster vaccination.

From eight days after challenge (D57 or 8 days post infection (dpi)) the imTLR1/2a, $7 / 8$ a and $9 a$ groups showed a specific antibody response at a comparable level to the O/Wcontrol group (anamnestic antibody response) with no significant difference between the different TLRa groups (Fig. 3A). On D57 and D59 (8 and 10 dpi) the skiTLRa groups showed a significantly lower antibody response compared to the O/W control group (Fig. 3B). The skiTLRa groups developed a specific antibody response from $14 \mathrm{dpi}$ (D63) in nearly all animals, with no significant difference between the TLRa groups.

A

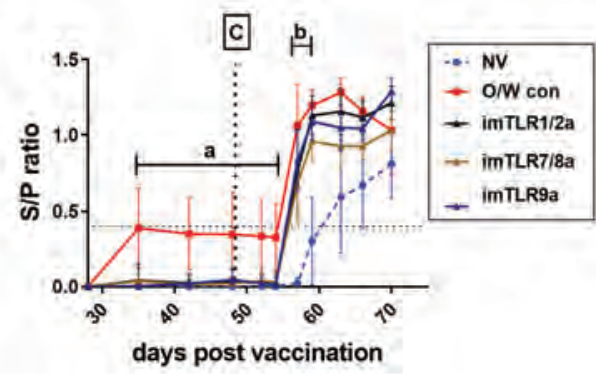

B

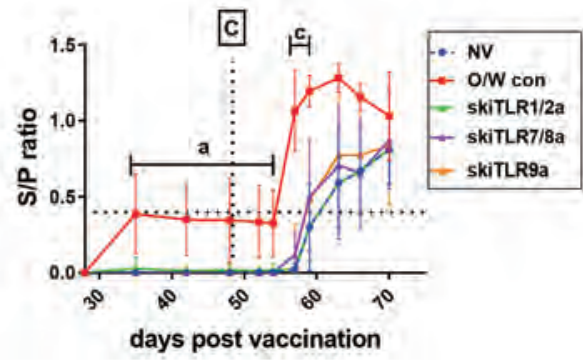

Figure 3: Kinetics of PRRSV specific antibody response after booster vaccination.

PRRSV antibody titers from booster vaccination (D28) till the end of the study determined by ELISA. (A) i.m. vaccinated Toll-like receptor agonist (imTLRa) groups compared to non-vaccinated (NV) group and oil-in-water group control (O/W con); (B) skin vaccinated skiTLRa groups compared to NV and O/W con groups; $C$ indicates challenge at D49; The dotted line marks the detection limit for the ELISA; Each data points represents the average of 6 animals \pm S.D. "a" indicates a significant difference $\left({ }^{*} p<0.05\right)$ between the O/W con group and the imTLRa/ skiTLRa/ NV groups; "b" indicates a significant difference between the NV group and the imTLRa/ O/W con groups; " $c$ " indicates a significant difference between the O/W con group and the skiTLRa/ NV groups.

\section{T-cell IFN- $\gamma$ response}

After primeand booster vaccination the cellular immune response was evaluated as number of IFN- $\gamma \mathrm{SCs}$ in the PBMCs after homologous in vitro re-stimulation with the challenge strain. Overall, in all groups there was a large individual variation between the animals in number of IFN- $\gamma$ SCs in non-stimulated and PRRSV stimulated samples. A large number of non-stimulated control samples contained IFN- $\gamma \mathrm{SC}$, indicating a non- 
antigen-specific IFN- $\gamma$ production. Only after booster vaccination (D42) the imTLR9a group showed significantly more non-specific IFN- $\gamma$ spots compared to the NV animals (Fig. 4A), additionally this was observed as a trend, albeit not significant $(0.05<p<0.10)$ in the O/W control group compared to the NV animals. This increased non-specific response compared to the NV group was not detectable in the skiTLR9a group, TLR1/2a and 7/8a experimental groups of either delivery routes.

There was no detectable antigen-specific cellular immune response on D21 (3 weeks after prime vaccination) as PBMCs produced no specifically induced IFN- $\gamma$ SCs (results not shown). On D42 (2 weeks after booster vaccination) only in the O/W control group, 3 of the 6 animals showed an increase in IFN- $\gamma$ SCs (Fig. 4B) compared to the non-stimulated control samples $(p=0.094)$, albeit not significant. None of the animals in the experimental TLRa groups showed a significant increase in IFN- $\gamma$ SCs compared to the control samples.
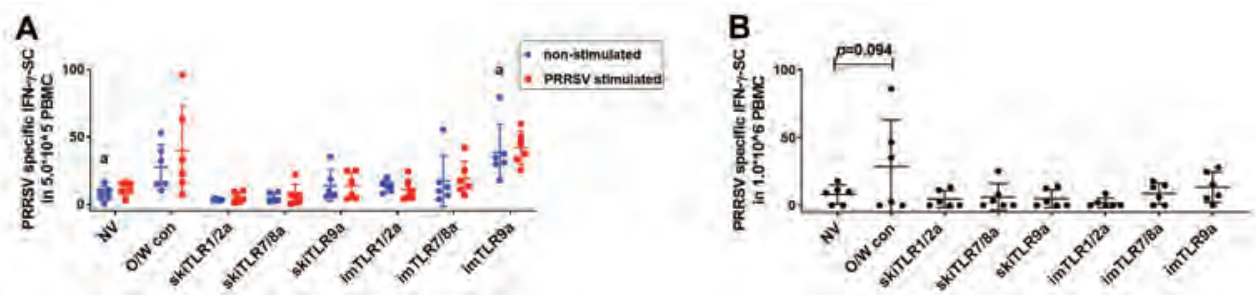

Figure 4: PRRSV specific T-cell IFN- $\gamma$ response two weeks after booster vaccination.

Number of IFN- $\gamma$ secreting cells (SC) after PBMC stimulation for $24 \mathrm{~h}$ with PBS (non-stimulated) or PRRSV(07V063). Response of non-vaccinated (NV) group and oil-in-water control (O/W con) group compared to the i.m. vaccinated Toll-like receptor agonists (imTLRa) groups and skin vaccinated skiTLRa groups measured two weeks after booster vaccination. Each symbol represents one animal (mean of triplicate wells) and the median of the group set $(n=6)$ is shown as a line; (A) Number IFN- $\gamma$ SCs in non-stimulated ( $\bullet$ background) and PRRSV stimulated samples ( $\square$ ); (B) Net IFN- $\gamma$ SCs with background subtracted from stimulated samples; "a" indicates a significant difference (* $p<0.05$ ) between non-stimulated samples of NV group and imTLR9a group.

\section{Serum cytokines 4 days after challenge}

To assess the influence of vaccination on the host response to viral challenge the serum IFN-a (anti-viral cytokine), IL-4 (Th2-type cytokine) levels and TNF and IL-6 levels (proinflammatory cytokines) were analysed 3 days after challenge. All animals showed a detectable IFN-a response with no significant difference between the groups (Fig. 5A). The O/W control group showed significant increased IL-4 level compared to the NV group with no significant difference between the two administration routes (Fig. 5B).

The median levels and number of animals producing the pro-inflammatory cytokines TNF and IL-6 were highest in the skiTLR7/8a group (Fig. 5C and D). TNF and IL-6 were equivalent to the non-vaccinated groups in all other groups. However, no significant differences in TNF and IL- 6 between groups were detected. 

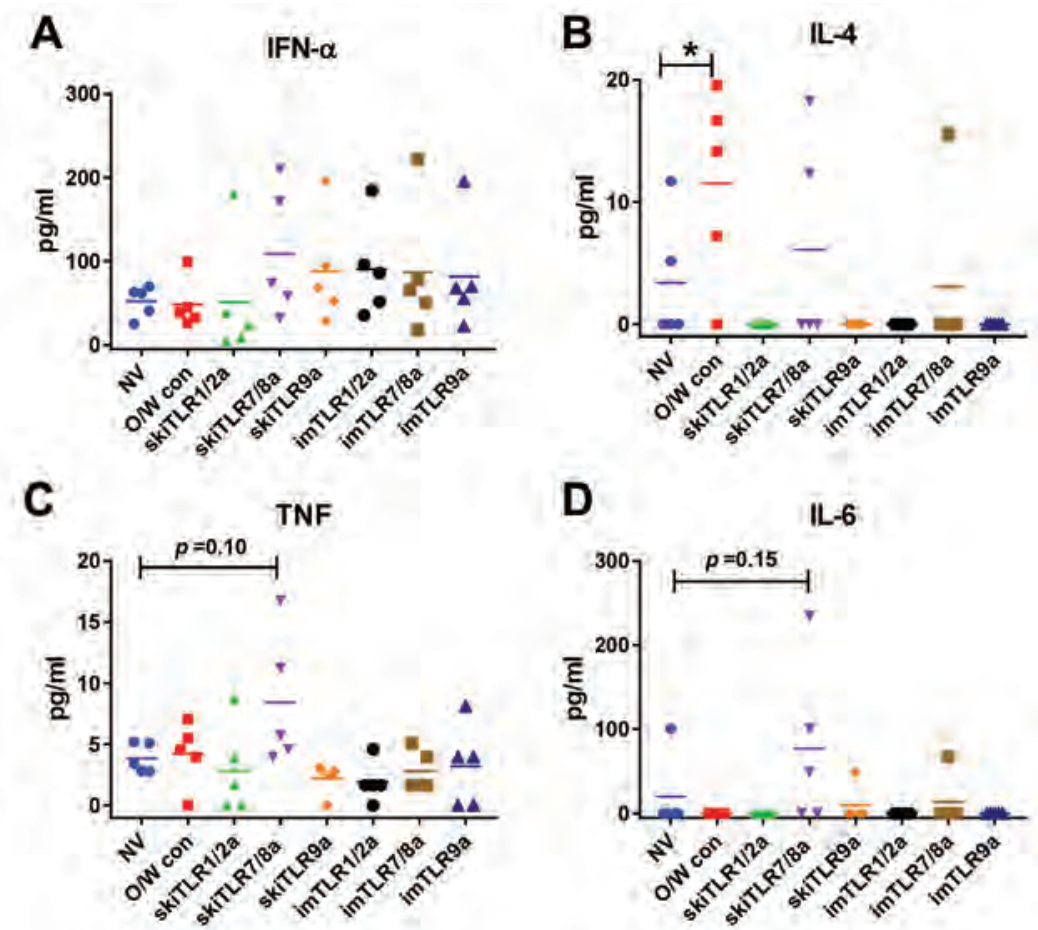

Figure 5: Serum cytokine concentrations 3 days after PRRSV challenge.

Serum cytokines $(\mathrm{pg} / \mathrm{ml})$ in non-vaccinated (NV) group and oil-in-water control (O/W con) group compared to the experimental i.m. vaccinated Toll-like receptor agonist (imTLRa) groups and skin vaccinated skiTLRa groups for IFN-a (A), IL-4 (B), TNF (C) and IL-6 (D). Each symbol represents one animal and the median is shown for the data set $(n=5) ;\left({ }^{*} p<0.05\right)$.

\section{Viremia in serum}

PRRSV 07 V063 was not detected in the serum before challenge. At D52 (3 dpi) PRRSV was detected in the serum of $92 \%$ of the animals and at 5 dpi all animals were viremic. At D54 (5dpi) the virus titer of only the skiTLR9a group was significantly lower than the NV group (Fig. 6A), this reduction of viremia was not observed for other time points in this group. At D63 (14 dpi) the O/W control and imTLR9a group showed a significantly lower virus titer compared to the NV animals (Fig. 6A and B); this decline was not observed in the other experimental TLRa groups. The imTLR9a group showed as well a significantly lower virus titer at 8 and $10 \mathrm{dpi}$.

The area under the curve (AUC) value was significantly lower for the imTLR9a and O/W control group compared to the NV animals (Fig. 6C), the other experimental TLRa groups did not show this decline. 


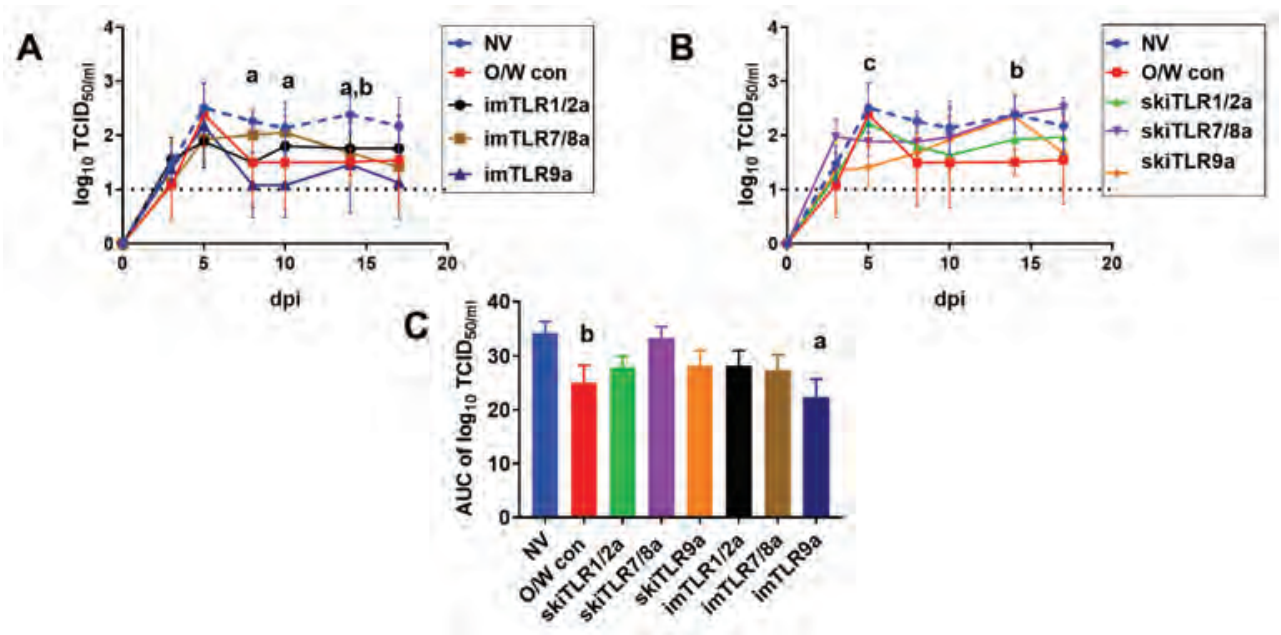

Figure 6: Clearance of PRRSV in serum after challenge.

Virus titers in serum (log10 TCID50/ml) determined by virus titration. (A) serum virus titers of i.m. vaccinated Toll-like receptor (imTLRa) groups compared to non-vaccinated (NV) group and oil-inwater control (O/W con) group (B); serum virus titers of skin vaccinated skiTLRa groups compared to non-NV group and O/W con group. Each data point represents the average of 6 animals \pm S.D. The dotted line marks the detection limit for virus titration; (C) Area under the curve (AUC) calculated for the whole group with the S.D. "a" indicates a significant difference $\left.{ }^{*} p<0.05\right)$ between imTLR9a group and NV group; "b" between O/W con group and NV group and "c" between the skiTLR9a group and the NV group.

\section{Pathology}

There were no or minimal gross pathological changes in the lungs 21 days after challenge in all groups. The O/W control group did not show any macroscopic changes in the lungs. In the other groups, individual scores ranged from $0 \%$ to $4 \%$ of affected lung surface (Fig. 7A). There was no significant difference in lung histopathology between the groups (Fig. $7 B, C$ and D). The gross lesions were predominantly located in the cranial and the middle lobe and were characterized by multifocal, irregular, slightly sunken red to tan areas. The lungs of all pigs displayed mild to moderate histopathological changes, although the gross changes were not evident. Compared to normal lung tissue histology of a pig with the same age and genetic background (Fig. 7E), we observed an interstitial pneumonia characterized by a mononuclear infiltrate of mainly macrophages and lymphocytes in the alveolar septa and around the blood vessels and bronchi and bronchioles (Fig. 7F and G). Also, dispersed the alveolar lumina contained a similar mononuclear infiltrate, which was occasionally admixed with cellular debris. 
A

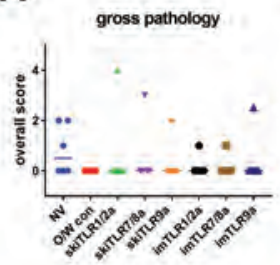

B

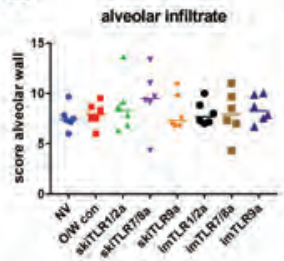

C

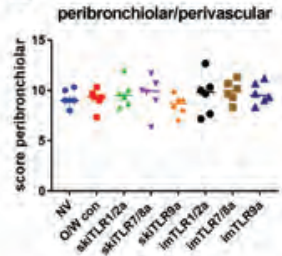

D

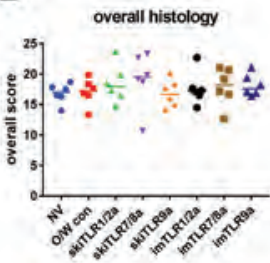

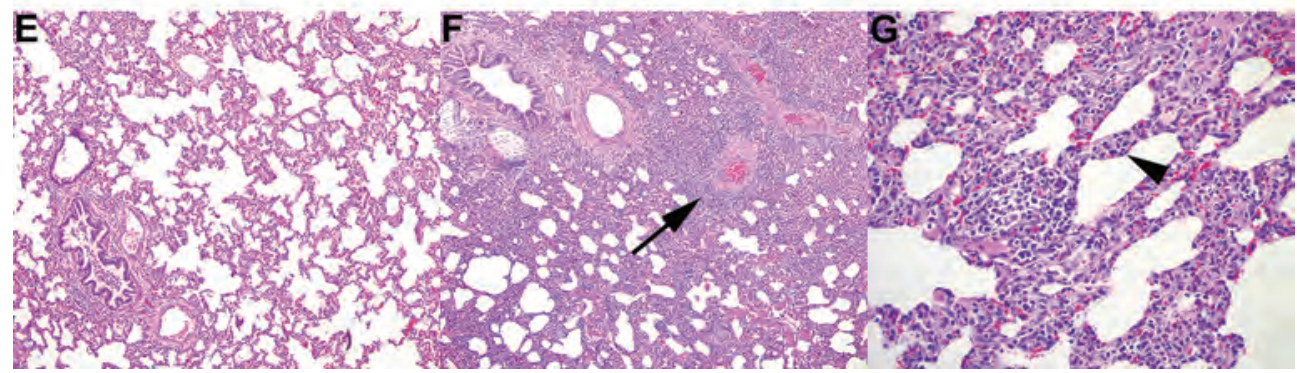

Figure 7: PRRSV lung pathology 21 days after challenge.

Lung tissue of non-vaccinated (NV) group and oil-in-water control (O/W con) group compared to the experimental i.m. vaccinated Toll-like receptor agonist (imTLRa) groups and skin vaccinated skiTLRa groups. (A) The extension of gross lung lesions calculated as proportion of total lung area; (B) Histologic lesions evaluated in the left cranial, medial and caudal lung lobe for infiltrate of mononuclear inflammatory cells in alveolar walls (score 0-5 for each lobe), (C) and around blood vessels, bronchi and bronchioli (score $0-5$ for each lobe) (D) combined to an overall histology score (score 0-30); Each symbol represents one animal and the median is shown for the data set $(n=6)$. (E) Normal lung tissue histology of a control pig with the same background and age (Haematoxylin and Eosin (HE) stain; magnification 200x); (B) interstitial pneumonia 21 days after PRRSV infection with a significant mononuclear infiltrate of mainly macrophages and lymphocytes around blood vessels and bronchi and bronchiole (arrow) (magnificantion 200x) ; (C) alveolar walls are thickened by the same type of inflammatory cells (arrowhead) (magnification 400x).

\section{Association between specific humoral and cellular immune response and viral clearance}

The time-points with the most prominent immune response (humoral D35 and D57; cellular D42) and the time-point with the most significant reduction of vireamie (D63) were used to investigate the association between specific immune response and viral clearance. This association between immunogenicity and efficacy was independent of the vaccine adjuvant and administration route (all animal were included, as well the NV group). There was a significant association of the humoral immune response one week after booster vaccination (D35) and one week after challenge (D57) with the cellular immune response two weeks after booster vaccination (D42) (Fig. 8A and B). However, no significant association was found between the viral clearance (D63) and the specific humoral or cellular immune response (Fig. $8 \mathrm{C}$ and D). 

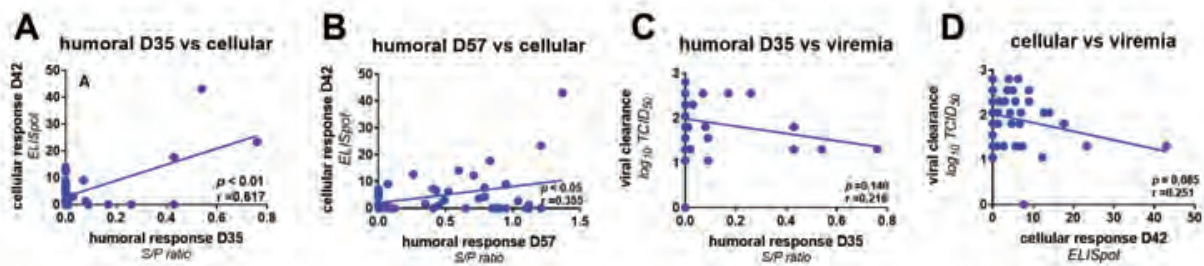

Figure 8: Association between PRRSV specific humoral and cellular immune response and viral clearance.

Scatter plots of humoral immune response on D35 (one week after booster vaccination) and D57 (one week after challenge) versus cellular immune response on D42 (two weeks after booster vaccination)(A and B); Scatter plots of viral clearance on D63 (15 days after challenge) versus cellular and humoral immune response (C and D). All animals indepent of vaccination an administration route, were included in this association.

\section{Discussion}

PRRSV is an important pathogen resulting in large scale health issues in pigs with major economic impact, therefore development of effective inactivated vaccines is highly desirable. Inadequate specific immune responses, especially cell-mediated responses, are often described for iPRRSV vaccines as a cause for limited protective efficacy 9,11,12,57. TLRa activity links the innate and adaptive immune response by activation of APCs and has a potency to enhance cell-mediated responses and therefore could be a suitable method to improve the efficacy of iPRRSV vaccines. Three different TLRa were selected based on porcine in vitro study results ${ }^{19,20}$ and this selection was supported by positive immunogenicity results from in vivo studies with inactivated vaccines in pigs, mice and humans ${ }^{21,25,28}$. The skin, which harbours a large number of APCs, was included as administration route to explore the potency of the different TLRa to a broader extent. Here, we examined the adjuvant potency of TLR1/2a (Pam3Cys), TLR7/8a (R848) or TLR9 (CpG ODN) to increase the iPRRSV vaccine response compared to an established O/W control adjuvant.

We found that none of the TLRa was able to induce a detecable specific immune response after booster vaccination for either delivery routes. However, the imTLR9a showed reduction of vireamia, indicating efficacy for $\mathrm{CpG}$ ODN as an injected adjuvant. As we only observed a reduction of the viremia in the i.m. TLR9a group, we consider that this result was most likely induced by the TLR9a as it was not observed in the other experimental groups with TLRa and the iPRRSV antigen. In the study design we did not include an iPRRSV only group as we were interested in the vaccine formulation. We anticipated that the iPRRSV antigen would be insufficient to show efficacy and/or an immune response on its own, as it is common, that inactivated viral vaccines are administered with an adjuvant.

Vaccine immunogenicity was measured by production of specific IgG antibodies in the serum and specific IFN- ${ }^{+}$T-cell response after vaccination. The ELISA showed only 
in the O/W control group a specific antibody response before challenge. These early antibodies often appear within one week after booster vaccination, however there is evidence that they play no significant role in PRRSV protection ${ }^{58}$. Virus neutralizing antibodies appear at a later time-point and their presence before challenge appears to correlate with passive protection ${ }^{59}$. In our study the experimental TLRa groups lacked the induction of any measurable specific antibody response before challenge, therefore the neutralizing antibodies were not measured.

Three days after challenge the IL-4 serum level was measured as an indicator of a Th2 or humoral immune response. Only in the O/W control group 4 of the 5 animals showed a clear IL-4 response at 3 days after challenge, supporting the specific antibody response after booster vaccination in this group as observed in other PRRSV vaccine studies ${ }^{60,61}$. However, serum IL-4 is often difficult to detect and the significance of this cytokine response for porcine B-cell stimulation is under debate ${ }^{62}$. In our study 2 of the 5 nonvaccinated animals also showed detectable IL-4 levels and in addition the serum before challenge was not analysed. Our study did not include an uninfected control group to measure baseline cytokines as our aim was to investigate response differences between vaccines. Therefore the IL-4 results should be interpreted with caution as a reflection of the humoral response.

An enhanced specific IFN- $\gamma$ response after booster vaccination and IFN-a response after challenge could contribute to more effective iPRRSV vaccines as insufficient T-cell responses and cytokine responses most likely play a pivotal role in the delayed adaptive immune response and clearance of PRRSV ${ }^{59,63}$. In our study the experimental TLR agonist groups did not enhance antigen-specific IFN- $\gamma$ cell-mediated response after booster and the anti-viral IFN- $a$ response after challenge. However, an increase in non-specific IFN- $\gamma$ production in the imTLR9a group was noticed in the ELISpot, which was most likely induced by activation of innate immune cells. This non-specific Th1-skewed response was also observed in a iPRRSV study with a different adjuvant system ${ }^{10}$, which induced no protective immunity. Interestingly, in our study only the imTLR9a group with this nonspecific IFN- $\gamma$ production also showed a reduction of viremia. This suggests that this group was able to induce a protective immune response, most likely supported by the nonspecific stimulation as measured in the ELISpot and the anamnestic antibody response after challenge. We consider this anamnestic antibody response suggestive for the added effect of the TLR agonist, although an iPRRSV only vaccination group was not included and we cannot fully exclude the contribution of iPRRSV antigen on its own.

Besides reduction of viremia, vaccine efficacy was assessed after challenge by reduction of both clinical signs and lung pathology. Within 6 days after challenge all animals were viremic. However, the PRRSV challenge did not induce any clinical signs or fever in the non-vaccinated animals. This mild clinical course has been described before for the 07V063 strain ${ }^{11,45}$. The lung pathology was determined 21 days after challenge and the histologic changes were generally mild to moderate and characteristic for PRRSV 
infection ${ }^{56}$. This mild lung pathology could be due to the time of necropsy (day 21 postinfection), as lung lesions are most prominent at 7-10 days post infection ${ }^{55}$. Overall, we can conclude that the reduction of the viremia in the O/W control and imTLR9a group did not result in reduced lung pathology compared to NV group at 21 days post-infection. However, we cannot exclude that at an earlier time-point after infection there could have been differences in lung pathology.

Skin delivery with DMN-patches did not influence the vaccine immunogenicity compared to the i.m route. However, the imTLRa groups were able to induce an anamnestic antibody response after challenge, which was lower in the skiTLRa groups and only the imTLR9a group showed significant vaccine efficacy after challenge. We did observe a transient, but significant reduction of viremia at $5 \mathrm{dpi}$ in the skiTLR9a group. However, lower viral titers were not detected at other time-points after challenge and AUC for the skiTLR9a was not significantly reduced compared to the NV group. We interpret this finding that an immune response was induced in this group that was capable of retarding early virus growth, however it was insufficient to protect the host over time from continued and increased virus proliferation. A porcine influenza study with skin vaccination demonstrated as well that TLR9a (CpG ODN) ${ }^{64}$ intradermally in pigs promoted the antigen-specific $A b$ responses, significantly but quite weakly. Overall, it is an interesting finding that TLR9a by both the i.m. and skin routes demonstrated the strongest efficacy potential.

It is unknown why the skin route was not able to benefit from the high number of accessible APCs in the skin to induce a comparable immune response and vaccine efficacy as seen in the imTLRa groups. One explanation could be a lower vaccine bioavailability by the skin route due to partially inserted DMN. It is commonly appreciated in the microneedle field that less than $100 \%$ of the cargo is delivered into skin; some reports where antigen is coated onto silicon microneedles report as little as $7 \%$ being delivered into skin ${ }^{65}$. A change in patch design, whereby the DMN contain the vaccine and adjuvant in the tips of the DMN only, compared to homogenous distribution throughout the length of the DMN could facilitate an increased dose delivery in future studies.

Another route-dependent finding was the local skin redness in skiTLR7a group, which was less prominent or absent in the skiTLR1/2a and 9a groups. This skin reaction is a strong indication for overall successful DMN-patch application and as well a side effect for topical administration of TLR7a Resiquimod (R848) as observed in other studies ${ }^{66,67}$. These side effects were considered negligible compared to the immunostimulatory properties ${ }^{33,68}$ of the TLR7/8a. In our study this difference in local immune response between the different TLR agonist did not result in a more prominent antigen-specific immune response, as assessed by antibody ELISA or IFN- $\gamma$ ELISpot or anamnestic immune response after challenge. However, other specific immune responses, such as tissue-specific $T$ and/or $B$ memory or mucosal responses, which did not contribute to protection, may have been induced but were not evaluated. 
No correlation was observed between the immune responses that were evaluated and protection against virus challenge. This indicates that other immune responses, which were not evaluated, were repsonsible for efficacy. These responses could include non-antigen specific innate immunity that may have been still active 2 weeks after the booster immunization and/or memory immune responses that were quickly re-activated after challenge but not assessed in our assays. Interestingly, we found in the serum of skiTLR7a group a trend for a higher level for the pro-inflammatory cytokines TNF and IL-6, post-challenge, which could be correlated to the skin reaction. Overall, the two different delivery routes showed only post-challenge differences in efficacy for TLR9a.

We anticipated that selected TLR agonists would have stimulated the production of pro-inflammatory cytokines/chemokines and type I IFNs supporting the development of an adaptive immune response in combination with the iPRRSV antigen. TLR7a and TLR9a combined with high pathogenic iPRRSV-2 antigen in a comparable setting 25,28 although with a different TLR7a, were able to enhance these specific immune responses and showed enhanced protection after homologous challenge. PRRSV-1 and PRRSV-2 are highly different strains (around 60\% homologous at genome level) ${ }^{69}$ and the use of iPRRSV-1 antigen might have provoked that our selected TLRa-antigen combinations were less successful. As well, we have to consider that the innate immune response directly after vaccination was not evaluated and it is unclear to what extent actual TLR activation occurred. We cannot exclude that the dose of the different TLRa was not optimal for the i.m/ and or the skin vaccination. As especially for the patches, no previous experiments have been performed and our reference dose for the different TLRa was based on different animal species and delivery routes combined with the maximum dose which could be incorporated in the DMN- patches. However, the O/W reference adjuvant was able to induce a specific immune response with iPRRSV-1 in combination with reduction of viremia. Possibly a different adjuvant system where different combinations of TLR agonists are combined with e.g. O/W adjuvant, liposomes ${ }^{70,71}$ or plasmids ${ }^{27}$ could enhance the vaccine properties and would be a direction for further research.

\section{Conclusion}

TLR 1/2a, 7/8a or 9a incorporated as adjuvants in iPRRSV $07 V 063$ antigen based vaccine were unable to induce a measurable specific immune response after booster vaccination and only the imTLR9a group induced reduction of viremia after homologous challenge, supported by non-antigen-specific IFN- $\gamma$ production after booster vaccination and an anamnestic antibody response after challenge. Further research is needed with different adjuvant systems, dosing regimens and combinations of TLR agonists and delivery routes to explore the potentials of TLRa as adjuvant for porcine vaccines with a preference for TLR9a. 


\section{Conflict of interest statement}

None of the authors have any potential conflict of interest regarding or related to this work.

\section{Acknowledgments}

The authors thank Artur Summerfield for his advice regarding adjuvant selection as partner of Horizon 2020 Programme. Ivan Trus and Lisette Ruuls are acknowledged for technical assistance. The animal caretakers are thanked for collection of all samples and providing optimal housing conditions for the pigs used in this experiment. Conor O'Mahony at the Tyndall National Institute, UCC is acknowledged for supplying PDMS moulds. This project has received funding from the European Union's Horizon 2020 Programme for research, technological development and demonstration under the Grant Agreement $n^{\circ} 633184$. This publication reflects the views only of the author, and not the European Commission (EC). The EC is not liable for any use that may be made of the information contained herein. 


\section{References}

1 Nathues, H. et al. Cost of porcine reproductive and respiratory syndrome virus at individual farm level - An economic disease model. Preventive veterinary medicine 142, 16-29, doi:10.1016/j.prevetmed.2017.04.006 (2017).

2 Nieuwenhuis, N., Duinhof, T. F. \& van Nes, A. Economic analysis of outbreaks of porcine reproductive and respiratory syndrome virus in nine sow herds. The Veterinary record 170, 225, doi:10.1136/vr.100101 (2012).

3 King, A. M. Q. et al. Changes to taxonomy and the International Code of Virus Classification and Nomenclature ratified by the International Committee on Taxonomy of Viruses (2018). Archives of virology, doi:10.1007/s00705-018-3847-1 (2018).

4 Stadejek, T. et al. Definition of subtypes in the European genotype of porcine reproductive and respiratory syndrome virus: nucleocapsid characteristics and geographical distribution in Europe. Archives of virology 153, 1479-1488, doi:10.1007/s00705-008-0146-2 (2008).

5 Nelsen, C. J., Murtaugh, M. P. \& Faaberg, K. S. Porcine reproductive and respiratory syndrome virus comparison: divergent evolution on two continents. Journal of virology 73, 270-280 (1999).

6 Brar, M. S., Shi, M., Murtaugh, M. P. \& Leung, F. C. Evolutionary diversification of type 2 porcine reproductive and respiratory syndrome virus. The Journal of general virology 96, 1570-1580, doi:10.1099/vir.0.000104 (2015).

7 Kappes, M. A. \& Faaberg, K. S. PRRSV structure, replication and recombination: Origin of phenotype and genotype diversity. Virology 479-480, 475-486, doi:10.1016/j.virol.2015.02.012 (2015).

8 Nan, Y. et al. Improved Vaccine against PRRSV: Current Progress and Future Perspective. Frontiers in microbiology 8, 1635, doi:10.3389/fmicb.2017.01635 (2017).

9 Renukaradhya, G. J., Meng, X. J., Calvert, J. G., Roof, M. \& Lager, K. M. Inactivated and subunit vaccines against porcine reproductive and respiratory syndrome: Current status and future direction. Vaccine 33, 3065-3072, doi:10.1016/j.vaccine.2015.04.102 (2015).

10 Zuckermann, F. A. et al. Assessment of the efficacy of commercial porcine reproductive and respiratory syndrome virus (PRRSV) vaccines based on measurement of serologic response, frequency of gammaIFN-producing cells and virological parameters of protection upon challenge. Veterinary microbiology 123, 69-85, doi:10.1016/j.vetmic.2007.02.009 (2007).

11 Geldhof, M. F. et al. Comparison of the efficacy of autogenous inactivated Porcine Reproductive and Respiratory Syndrome Virus (PRRSV) vaccines with that of commercial vaccines against homologous and heterologous challenges. BMC veterinary research 8, 182, doi:10.1186/1746-6148-8-182 (2012).

12 Vanhee, M., Delputte, P. L., Delrue, I., Geldhof, M. F. \& Nauwynck, H. J. Development of an experimental inactivated PRRSV vaccine that induces virus-neutralizing antibodies. Veterinary research 40, 63, doi:10.1051/vetres/2009046 (2009).

13 Takeda, K. \& Akira, S. Toll-like receptors in innate immunity. International immunology 17, 1-14, doi:10.1093/ intimm/dxh186 (2005).

14 Maisonneuve, C., Bertholet, S., Philpott, D. J. \& De Gregorio, E. Unleashing the potential of NOD- and Tolllike agonists as vaccine adjuvants. Proceedings of the National Academy of Sciences of the United States of America 111, 12294-12299, doi:10.1073/pnas.1400478111 (2014). 
15 Toussi, D. N. \& Massari, P. Immune Adjuvant Effect of Molecularly-defined Toll-Like Receptor Ligands. Vaccines 2, 323-353, doi:10.3390/vaccines2020323 (2014).

16 Steinhagen, F., Kinjo, T., Bode, C. \& Klinman, D. M. TLR-based immune adjuvants. Vaccine 29, 3341-3355, doi:10.1016/j.vaccine.2010.08.002 (2011).

17 Akira, S. \& Takeda, K. Toll-like receptor signalling. Nature reviews. Immunology 4, 499-511, doi:10.1038/ nri1391 (2004).

18 Uenishi, H. \& Shinkai, H. Porcine Toll-like receptors: the front line of pathogen monitoring and possible implications for disease resistance. Developmental and comparative immunology 33, 353-361, doi:10.1016/j. dci.2008.06.001 (2009).

19 Vreman, S. et al. Neonatal porcine blood derived dendritic cells subsets show activation after TLR2 or TLR9 stimulation. Developmental and comparative immunology, doi:10.1016/j.dci.2018.03.012 (2018).

20 Auray, G. et al. Characterization and Transcriptomic Analysis of Porcine Blood Conventional and Plasmacytoid Dendritic Cells Reveals Striking Species-Specific Differences. Journal of immunology 197, 4791-4806, doi:10.4049/jimmunol.1600672 (2016).

21 Basto, A. P. \& Leitao, A. Targeting TLR2 for vaccine development. Journal of immunology research 2014, 619410, doi:10.1155/2014/619410 (2014).

22 Scheiermann, J. \& Klinman, D. M. Clinical evaluation of CpG oligonucleotides as adjuvants for vaccines targeting infectious diseases and cancer. Vaccine 32, 6377-6389, doi:10.1016/j.vaccine.2014.06.065 (2014).

23 Van der Stede, Y., Verdonck, F., Vancaeneghem, S., Cox, E. \& Goddeeris, B. M. CpG-oligodinucleotides as an effective adjuvant in pigs for intramuscular immunizations. Veterinary immunology and immunopathology 86, 31-41 (2002).

24 Knudsen, N. P. et al. Different human vaccine adjuvants promote distinct antigen-independent immunological signatures tailored to different pathogens. Scientific reports 6, 19570, doi:10.1038/ srep19570 (2016).

25 Zhang, L. et al. Toll-like receptor ligands enhance the protective effects of vaccination against porcine reproductive and respiratory syndrome virus in swine. Veterinary microbiology 164, 253-260, doi:10.1016/j. vetmic.2013.02.016 (2013).

26 Du, Y. et al. Synthetic Toll-like receptor 7 ligand inhibits porcine reproductive and respiratory syndrome virus infection in primary porcine alveolar macrophages. Antiviral research 131, 9-18, doi:10.1016/j. antiviral.2016.04.005 (2016).

27 Quan, Z. et al. Plasmid containing CpG oligodeoxynucleotides can augment the immune responses of pigs immunized with porcine reproductive and respiratory syndrome killed virus vaccine. Veterinary immunology and immunopathology 136, 257-264, doi:10.1016/j.vetimm.2010.03.015 (2010).

Linghua, Z., Xingshan, T. \& Fengzhen, Z. Vaccination with porcine reproductive and respiratory syndrome killed virus vaccine and immunostimulatory oligodeoxynucleotides induces specific immunity in piglets. Vaccine 25, 1735-1742, doi:10.1016/j.vaccine.2006.11.033 (2007).

Stadejek, T., Stankevicius, A., Murtaugh, M. P. \& Oleksiewicz, M. B. Molecular evolution of PRRSV in Europe: current state of play. Veterinary microbiology 165, 21-28, doi:10.1016/j.vetmic.2013.02.029 (2013).

30 Zhang, L., Wang, W. \& Wang, S. Effect of vaccine administration modality on immunogenicity and efficacy. Expert review of vaccines 14, 1509-1523, doi:10.1586/14760584.2015.1081067 (2015). 
31 Vrdoljak, A. et al. Induction of broad immunity by thermostabilised vaccines incorporated in dissolvable microneedles using novel fabrication methods. Journal of controlled release : official journal of the Controlled Release Society 225, 192-204, doi:10.1016/j.jconrel.2016.01.019 (2016).

32 Ouyang, K. et al. Comparative analysis of routes of immunization of a live porcine reproductive and respiratory syndrome virus (PRRSV) vaccine in a heterologous virus challenge study. Veterinary research 47, 45, doi:10.1186/s13567-016-0331-3 (2016).

33 van Aalst, S. et al. Routing dependent immune responses after experimental R848-adjuvated vaccination. Vaccine 36, 1405-1413, doi:10.1016/j.vaccine.2018.01.077 (2018).

34 Marshall, S., Sahm, L. J. \& Moore, A. C. The success of microneedle-mediated vaccine delivery into skin. Hum. Vaccin. Immunother., 0, doi:10.1080/21645515.2016.1171440 (2016).

35 Ferrari, L. et al. Lymphocyte activation as cytokine gene expression and secretion is related to the porcine reproductive and respiratory syndrome virus (PRRSV) isolate after in vitro homologous and heterologous recall of peripheral blood mononuclear cells (PBMC) from pigs vaccinated and exposed to natural infection. Veterinary immunology and immunopathology 151, 193-206, doi:10.1016/j.vetimm.2012.11.006 (2013).

$36 \mathrm{Krejci}$, J. et al. Effects of adjuvants on the immune response of pigs after intradermal administration of antigen. Research in veterinary science 94, 73-76, doi:10.1016/j.rvsc.2012.07.021 (2013).

37 Eble, P. L., Weerdmeester, K., van Hemert-Kluitenberg, F. \& Dekker, A. Intradermal vaccination of pigs against FMD with $1 / 10$ dose results in comparable vaccine efficacy as intramuscular vaccination with a full dose. Vaccine 27, 1272-1278, doi:10.1016/j.vaccine.2008.12.011 (2009).

38 Le Luduec, J. B. et al. Intradermal vaccination with un-adjuvanted sub-unit vaccines triggers skin innate immunity and confers protective respiratory immunity in domestic swine. Vaccine 34, 914-922, doi:10.1016/j.vaccine.2015.12.058 (2016).

39 Martelli, P.et al. Systemic and local immune response in pigs intradermally and intramuscularly injected with inactivated Mycoplasma hyopneumoniae vaccines. Veterinary microbiology 168, 357-364, doi:10.1016/j. vetmic.2013.11.025 (2014).

40 Magiri,R.B.etal.Responseofimmuneresponsegenestoadjuvantspoly[di(sodiumcarboxylatoethylphenoxy) phosphazene] (PCEP), CpG oligodeoxynucleotide and emulsigen at intradermal injection site in pigs. Veterinary immunology and immunopathology 175, 57-63, doi:10.1016/j.vetimm.2016.05.003 (2016).

41 McKay, P. F. et al. TLR4 and TLR7/8 Adjuvant Combinations Generate Different Vaccine Antigen-Specific Immune Outcomes in Minipigs when Administered via the ID or IN Routes. PloS one 11, e0148984, doi:10.1371/journal.pone.0148984 (2016).

42 Weldon, W. C. et al. Effect of adjuvants on responses to skin immunization by microneedles coated with influenza subunit vaccine. PloS one 7, e41501, doi:10.1371/journal.pone.0041501 (2012).

43 Van Doorsselaere, J., Geldhof, M., Nauwynck, H. J. \& Delputte, P. L. Characterization of a circulating PRRSV strain by means of random PCR cloning and full genome sequencing. Virology journal 8, 160, doi:10.1186/1743-422x-8-160 (2011).

44 Karniychuk, U.U.etal. Pathogenesis and antigenic characterization of a new East European subtype 3 porcine reproductive and respiratory syndrome virus isolate. BMC veterinary research 6, 30, doi:10.1186/1746-61486-30 (2010). 
45 Weesendorp, E. et al. Comparative analysis of immune responses following experimental infection of pigs with European porcine reproductive and respiratory syndrome virus strains of differing virulence. Veterinary microbiology 163, 1-12, doi:10.1016/j.vetmic.2012.09.013 (2013).

46 Van Breedam, W. et al. Porcine reproductive and respiratory syndrome virus (PRRSV)-specific mAbs: supporting diagnostics and providing new insights into the antigenic properties of the virus. Veterinary immunology and immunopathology 141, 246-257, doi:10.1016/j.vetimm.2011.03.008 (2011).

47 Shakya, N., Sane, S. A., Shankar, S. \& Gupta, S. Effect of Pam3Cys induced protection on the therapeutic efficacy of miltefosine against experimental visceral leishmaniasis. Peptides 32, 2131-2133, doi:10.1016/j. peptides.2011.09.014 (2011).

48 Salabert, N. et al. Intradermal injection of an anti-Langerin-HIVGag fusion vaccine targets epidermal Langerhans cells in nonhuman primates and can be tracked in vivo. European journal of immunology 46, 689-700, doi:10.1002/eji.201545465 (2016).

49 Linghua, Z., Yong, G., Xingshan, T. \& Fengzhen, Z. Co-administration of porcine-specific CpG oligodeoxynucleotide enhances the immune responses to pseudorabies attenuated virus vaccine in newborn piglets in vivo. Developmental and comparative immunology 30, 589-596, doi:10.1016/j. dci.2005.07.005 (2006).

50 Diaz, l. et al. Comparison of two commercial enzyme-linked immunosorbent assays for the diagnosis of Porcine reproductive and respiratory syndrome virus infection. Journal of veterinary diagnostic investigation : official publication of the American Association of Veterinary Laboratory Diagnosticians, Inc 24, 344-348, doi:10.1177/1040638711435804 (2012).

51 Mair, K. H. et al. Carbopol improves the early cellular immune responses induced by the modified-life vaccine Ingelvac PRRS(R) MLV. Veterinary microbiology 176, 352-357, doi:10.1016/j.vetmic.2015.02.001 (2015).

52 Mokhtar, H. et al. The Non-structural Protein 5 and Matrix Protein Are Antigenic Targets of T Cell Immunity to Genotype 1 Porcine Reproductive and Respiratory Syndrome Viruses. Frontiers in immunology 7, 40, doi:10.3389/fimmu.2016.00040 (2016).

53 van der Linden, I. F., Voermans, J. J., van der Linde-Bril, E. M., Bianchi, A. T. \& Steverink, P. J. Virological kinetics and immunological responses to a porcine reproductive and respiratory syndrome virus infection of pigs at different ages. Vaccine 21, 1952-1957 (2003).

54 Reed, L. \& Muench, H. A simple method of estimating fifty percent endpoints. Am J Hyg 27, $493-497$ (1938).

55 Halbur, P. G. et al. Comparison of the pathogenicity of two US porcine reproductive and respiratory syndrome virus isolates with that of the Lelystad virus. Veterinary pathology 32, 648-660, doi:10.1177/030098589503200606 (1995).

56 Weesendorp, E., Rebel, J. M., Popma-De Graaf, D. J., Fijten, H.P.\& Stockhofe-Zurwieden, N. Lung pathogenicity of European genotype 3 strain porcine reproductive and respiratory syndrome virus (PRRSV) differs from that of subtype 1 strains. Veterinary microbiology 174, 127-138, doi:10.1016/j.vetmic.2014.09.010 (2014).

57 Charerntantanakul, W. Adjuvants for porcine reproductive and respiratory syndrome virus vaccines. Veterinary immunology and immunopathology 129, 1-13, doi:10.1016/j.vetimm.2008.12.018 (2009). 
58 Labarque, G. G., Nauwynck, H. J., Van Reeth, K. \& Pensaert, M. B. Effect of cellular changes and onset of humoral immunity on the replication of porcine reproductive and respiratory syndrome virus in the lungs of pigs. The Journal of general virology 81, 1327-1334, doi:10.1099/0022-1317-81-5-1327 (2000).

59 Loving, C. L., Osorio, F. A., Murtaugh, M. P. \& Zuckermann, F. A. Innate and adaptive immunity against Porcine Reproductive and Respiratory Syndrome Virus. Veterinary immunology and immunopathology 167, 1-14, doi:10.1016/j.vetimm.2015.07.003 (2015).

60 Chen, C. et al. Synthetic B- and T-cell epitope peptides of porcine reproductive and respiratory syndrome virus with Gp96 as adjuvant induced humoral and cell-mediated immunity. Vaccine 31, 1838-1847, doi:10.1016/j.vaccine.2013.01.049 (2013).

61 Wang, G. et al. Immune responses in piglets infected with highly pathogenic porcine reproductive and respiratory syndrome virus. Veterinary immunology and immunopathology 142, 170-178, doi:10.1016/j. vetimm.2011.05.004 (2011).

62 Murtaugh, M. P., Johnson, C. R., Xiao, Z., Scamurra, R. W. \& Zhou, Y. Species specialization in cytokine biology: is interleukin-4 central to the $\mathrm{T}(\mathrm{H}) 1-\mathrm{T}(\mathrm{H}) 2$ paradigm in swine? Developmental and comparative immunology 33, 344-352, doi:10.1016/j.dci.2008.06.014 (2009). van Reeth, K. \& Nauwynck, H. Proinflammatory cytokines and viral respiratory disease in pigs. Veterinary research 31, 187-213, doi:10.1051/vetres:2000113 (2000).

Bernelin-Cottet, C. et al. A Universal Influenza Vaccine Can Lead to Disease Exacerbation or Viral Control Depending on Delivery Strategies. Frontiers in immunology 7, 641, doi:10.3389/fimmu.2016.00641 (2016).

65 Crichton, M. L. et al. The effect of strain rate on the precision of penetration of short densely-packed microprojection array patches coated with vaccine. Biomaterials 31, 4562-4572, doi:10.1016/j. biomaterials.2010.02.022 (2010).

66 Meyer, T., Surber, C., French, L. E. \& Stockfleth, E. Resiquimod, a topical drug for viral skin lesions and skin cancer. Expert opinion on investigational drugs 22, 149-159, doi:10.1517/13543784.2013.749236 (2013).

67 Hengge, U. R. \& Ruzicka, T. Topical immunomodulation in dermatology: potential of toll-like receptor agonists. Dermatologic surgery : official publication for American Society for Dermatologic Surgery [et al.] 30, 1101-1112, doi:10.1111/j.1524-4725.2004.30335.x (2004).

68 Sauder, D. N. Immunomodulatory and pharmacologic properties of imiquimod. Journal of the American Academy of Dermatology 43, S6-11 (2000).

69 Van Doorsselaere, J. et al. Complete genome characterization of a East European Type 1 subtype 3 porcine reproductive and respiratory syndrome virus. Virus genes 44, 51-54, doi:10.1007/s11262-011-0665-x (2012).

70 McKee, A. S. \& Marrack, P. Old and new adjuvants. Current opinion in immunology 47, 44-51, doi:10.1016/j. coi.2017.06.005 (2017).

71 Neeland, M. R., Elhay, M. J., Nathanielsz, J., Meeusen, E. N. \& de Veer, M. J. Incorporation of CpG into a liposomal vaccine formulation increases the maturation of antigen-loaded dendritic cells and monocytes to improve local and systemic immunity. Journal of immunology 192, 3666-3675, doi:10.4049/ jimmunol.1303014 (2014). 


\section{Immune responses induced by inactivated porcine reproductive and respiratory syndrome virus (PRRSV) vaccine in neonatal pigs using different adjuvants}

Sandra Vreman', Norbert Stockhofe-Zurwieden', Ditta J. Popma-de Graaf', Huub F.J. Savelkoul'2, C. Barnier-Quer ${ }^{3}$, N. Collin ${ }^{3}$, Damien Collins ${ }^{4}$, Dennis McDaid ${ }^{4}$, Anne C. Moore ${ }^{5}$ and Johanna M.J. Rebel ${ }^{6}$

1 Wageningen Bioveterinary Research, Wageningen University \& Research, P.O. Box 29703, 2502 LS The Hague, The Netherlands

${ }^{2}$ Cell Biology \& Immunology group, Wageningen University \& Research P.O. Box 338, 6700 HA Wageningen, The Netherlands

${ }^{3}$ Vaccine Formulation Laboratory, University of Lausanne, Epalinges, Switzerland

${ }^{4}$ Xeolas, Pharmaceuticals, Dublin Ireland

${ }^{5}$ School of Biochemistry and Cell Biology, School of Pharmacy University College Cork, Cork, Ireland

${ }^{6}$ Wageningen Livestock Research, Wageningen University \& Research, The Netherlands 


\begin{abstract}
Vaccination of neonatal pigs could be supportive to prevent porcine reproductive and respiratory syndrome virus (PRRSV), which is an important porcine pathogen causing worldwide welfare and health problems in pigs of different age classes. However, neonatal immunity substantially differs to adults, thus different vaccines may be required in neonatal pigs. We examined if the immunogenicity and efficacy of inactivated PRRSV (iPRRSV) vaccines in neonatal pigs could be improved with adjuvants containing oil-in water (O/W) emulsions with or without Toll-like receptor (TLR) agonists and by altering the delivery route from intramuscular (i.m) to the skin. Three-day-old PRRSV-naïve piglets ( $n=54$, divided in 6 groups) received a prime vaccination and a booster vaccination four weeks later. The vaccine formulations consisted of different O/W emulsions (Montanide ${ }^{\text {TM }}$ ISA28RVG (ISA28), a squalene in water emulsion (SWE) for i.m. or a Stable Emulsion (SE) with squalene for skin vaccination) and/or a mixture of TLR1/2, 7/8 and 9 agonists (TLRa) combined with iPRRSV strain 07V063. These vaccines were delivered either i.m. (ISA28, SWE, TLRa or SWE+TLRa) or into the skin (skiSE+TLRa) with dissolving microneedle (DMN)-patches. All animals received a challenge with homologous PRRSV three weeks after booster vaccination. Specific antibodies, IFN- $\gamma$ production and viremia were measured at several time-points after vaccination and/or challenge, while lung pathology was studied at necropsy. After booster vaccination, only ISA28 induced a specific antibody response while a specific T-cell IFN- $\gamma$ response was generated in the SWE group, that was lower for ISA28, and absent in the other groups. This suggests that prime vaccination in neonates induced a specific immune response after booster vaccination, dependent on the emulsion formulation, but not dependent on the presence of the TLRa or delivery route. Despite the measured immune responses none of the vaccines showed any efficacy. Further research focused on the early immune response in draining lymph nodes is needed to elucidate the potential of TLRa in vaccines for neonatal pigs.
\end{abstract}




\section{Introduction}

Porcine reproductive and respiratory syndrome virus (PRRSV) is occurring globally and causes health and welfare problems with severe economic losses ${ }^{1,2}$. This positive-stranded RNA virus of the Arteriviridae causes abortions in sows, respiratory diseases and increased susceptibility to other infections in nursery and fattening pigs. Modified-live PRRSV based vaccines induces weak immune responses, however lack of cross-protection and reduced safety, because of spreading the vaccine virus strain, are points of shortcomings ${ }^{3}$.Therefore, inactivated PRRSV (iPRRSV) vaccines are preferred, however these iPRRSV vaccines induce little or no cellular and humoral immunity and induce only limited protection against infection ${ }^{4-6}$. Thus, there is a need for strong immuno-stimulators (adjuvants) to increase the efficacy of these iPRRSV vaccines ${ }^{7}$.

Vaccination of neonatal pigs ( $<1$-week-old) could be an effective strategy to increase protection at an early age and thereby decrease the number of PRRSV infections in young piglets ${ }^{8,9}$. New-born piglets depend heavily on their innate immune defence and need to develop their specific immune system after birth. The neonatal immune response in mammals is characterized by Th2 directed cytokine patterns and limited Th1 and cytotoxic T-cell responses ${ }^{10-12}$, combined with limited germinal centre B-cell and plasmacell responses ${ }^{13,14}$. Furthermore, milk-derived maternal antibodies ${ }^{15}$, could interfere with the development of an active immune response in different ways ${ }^{16-18}$. All these aspects could complicate iPRRSV vaccination of neonatal pigs. New adjuvants and formulations could enhance the immunogenicity of inactivated vaccines, but must be suitable for the neonatal immune system ${ }^{19,20}$.

Toll-like receptor (TLR) agonists and combination of TLR agonists with oil-in water $(\mathrm{O} / \mathrm{W})$ emulsions ${ }^{21}$, have shown to increase the Th1 directed immune response to improve vaccine efficacy in neonates ${ }^{22}$ and adults ${ }^{23}$. In vitro stimulation of porcine PBMCs with various TLR agonists demonstrated that TLR1/2, 7/8 and 9 agonists induced activation of antigen presenting cells (APCs), including dendritic cells (DCs), monocytes and B-cells in adult pigs ${ }^{24,25}$ and TLR1/2 and 9 agonists activated APCs in neonatal pigs ${ }^{26}$. In our previous vaccine study in 6-week-old pigs ${ }^{27}$, the iPRRSV vaccine was combined with individual TLR1/2, $7 / 8$ or 9 agonist as adjuvant. In that study, the individual TLR agonists did not generate a PRRSV-specific immune response when the vaccines were applied i.m. or by skin vaccination, however the O/W emulsion Montanide ${ }^{\mathrm{TM}}$ ISA28R VG (ISA28) induced humoral and cellular immunity and reduction of viremia. Thus, we selected ISA28 as reference O/W emulsion for this neonatal iPRSSV study. An O/W emulsion (SWE) combined with a mixture of TLR1/2, 7/8 and 9 agonists (TLRa) has been shown to elicit a clear Th 1 and innate immune response in adult pigs ${ }^{28}$ and therefore we hypothesised that this may be a candidate adjuvant for neonatal pigs.

Next to the antigen and the choice of adjuvant, the route of vaccine delivery can be important to induce an effective immune response ${ }^{29,30}$. The skin contains a large amount of APCs, which could be directly activated with skin vaccination to transport the antigen 
to the draining lymph node. This may result in an equal or enhanced immunological effectiveness compared to traditional i.m. delivery ${ }^{31-33}$. Other suggested advantages of skin vaccination are dose-sparing ${ }^{34}$, needle-free application ${ }^{35}$ and the induction of mucosal immune responses, which is preferred for respiratory diseases like PRRSV ${ }^{36,37}$.

In this study we assessed in vitro the cytokine production of porcine neonatal PBMCs that were stimulated with the individual TLR1/2 (Pam3Cys), $7 / 8$ (R848) or 9 (CPG) agonists or the TLRa mixture. We then examined the immune responses and the protection after challenge in 3-day-old PRRSV-naïve piglets that were vaccinated with iPRRSV vaccines. The vaccines contained different adjuvant formulations (O/W emulsion with or without TLRa) and were applied i.m. and through skin vaccination to overcome the potential weak immune response of neonatal pigs.

\section{Materials and methods}

\section{Animal procedures and Ethics}

All scientific procedures on animals in this study were executed according to the Dutch animal experimental and ethical requirements and the Dutch Central Authority for Scientific Procedures on Animals (CCD) has approved the project license application under Permit number: ADV401002015356.

\section{PBMC isolation and stimulation with Toll-like receptor agonists in neonatal pigs}

Heparin stabilized blood was collected from four $(n=4)$ three-day-old male piglets. Stabilized blood was diluted 1:1 with PBS within 2 hours after sampling and added to a Leucosep ${ }^{\mathrm{TM}}$ tube containing a $60 \%$ FICOLL-PAQUE $^{\mathrm{TM}}$ Plus density- gradient for PBMC isolation. Remaining red blood cells, which are often present after PBMC isolation in neonatal porcine blood, were lysed with Gibco $^{\text {TM }}$ ACK lysing buffer. Cells were inserted in 96-well plates with $0.5 \times 106$ cells/well in RPMI 1640 medium (Gibco ${ }^{\circ}$ ) with $10 \%$ foetal bovine serum. After $1 \mathrm{~h}$ of incubation, cells were stimulated with the same TLR agonists as used in the vaccine adjuvant: TLR1/2 agonist $(10 \mu \mathrm{g} / \mathrm{ml} \mathrm{Pam3Cys} \mathrm{L2000} \mathrm{from} \mathrm{EMC} \mathrm{micro-}$ collections), TLR7/8 agonist ( $5 \mu \mathrm{g} / \mathrm{ml}$ R848, Resiquimod from InvivoGen), TLR9 agonist ( $5 \mu \mathrm{g} / \mathrm{ml}$ CpG ODN-type A sequence D32, 5'-ggTGCGTCGACGCAGggggg-3', from Eurofins Genomics.), the TLR1/2a, $7 / 8$ and 9 agonist mixture (with $10 \mu \mathrm{g} / \mathrm{ml}, 5 \mu \mathrm{g} / \mathrm{ml}$ and $5 \mu \mathrm{g} / \mathrm{ml}$, respectively), the TLR1/2 and 9 agonist mixture $(10 \mu \mathrm{g} / \mathrm{ml}$ and $5 \mu \mathrm{g} / \mathrm{ml}$, respectively) or cells were left unstimulated as negative control.

\section{In vitro cytokine production measured by multiplex immunoassay}

PBMCs were stimulated for $24 \mathrm{~h}$ and supernatants of PBMC cultures were collected and frozen at $-80^{\circ} \mathrm{C}$ until analysis within 2 months after collection. Cytokine protein were measured with a multiplex Cytometric Bead Array (Cytokine \& chemokine 9-Plex Porcine ProcartaPlex ${ }^{\circ}$ Multiplex Immunoassay from eBioscience) according to the manufacturer's instructions and read on a Luminex $200^{\mathrm{TM}}$ (Luminex Corporation). Cytokine concentrations 
were determined using $x \mathrm{PONENT}^{\circ}$ software (Luminex Corporation). The detection limits of the measured cytokines were $0.72 \mathrm{pg} / \mathrm{ml}$ (IFN-a), $4.96 \mathrm{pg} / \mathrm{ml}$ (IFN- - ), $7.57 \mathrm{pg} / \mathrm{ml}$ (TNF), 8.20 $\mathrm{pg} / \mathrm{ml}$ (IL-10), $35 \mathrm{pg} / \mathrm{ml}$ (IL-12p40), $3.54 \mathrm{pg} / \mathrm{ml}$ (IL-1ß), $1.55 \mathrm{pg} / \mathrm{ml}$ (IL-4), $6.32 \mathrm{pg} / \mathrm{ml}$ (IL-6) and $12 \mathrm{pg} / \mathrm{ml}$ (IL-8).

\section{Vaccines}

All the vaccines (i.m. and DMN-patches) contained the same dose of binary ethylenimine (BEI)-inactivated PRRSV $07 V 063\left(1.0 \times 10^{8} \mathrm{TCID}_{50}\right)$ and were formulated with different adjuvants (Table 1). The iPRRSV-antigen was manufactured as described previously ${ }^{27}$. Within the study we used three different O/Wemulsions. Commercial available Montanide ${ }^{\mathrm{TM}}$ ISA 28R VG (kindly provided by SEPPIC), an O/W emulsion containing a combination of a mineral and non-mineral oil, was applied i.m. (ISA28). Two different O/W emulsion with squalene both with a similar chemical composition, however with different quantities and based on a different manufacturing process. The squalene based emulsion (SWE), used for the i.m vaccination, is developed and produced by the Vaccine Formulation Laboratory, and composed of $3.9 \%$ weight per volume $(\mathrm{w} / \mathrm{v})$ squalene, $0.5 \%(\mathrm{w} / \mathrm{v})$ Tween 80 and $0.5 \%$ $(w / v)$ Span 85$)^{38}$. The O/W stable emulsion (SE) with squalene used in the DMN-patch was made according to previously described methods ${ }^{39}$ and is composed of $3.5 \%$ volume per volume (v/v) squalene, 1\% (v/v) Tween 80 and $0.5 \%$ (v/v) Span 85).

Three groups were i.m immunised with an $\mathrm{O} / \mathrm{W}$ emulsion with or without TLR agonists mixture and iPRRSV. The ISA28 group received a solution containing 15\% volume per volume (v/v) Montanide ${ }^{\mathrm{TM}}$ ISA 28R VG with iPRRSV. The SWE group received iPRRSV in combination with $42 \%(\mathrm{v} / \mathrm{v})$ SWE. The third group received SWE with the three TLRa by the i.m. route (SWE+TLRa). For this vaccine 42\% (v/v) SWE was mixed with $80 \mu \mathrm{g}$ of each of the three TLR agonists: Pam3Cys, R848, Resiquimod and CpG ODN-type A (25\% (v/v) for the TLRa). Alternatively, animals received iPRRSV with the TLR agonist mixture only (TLRa).

For the skin vaccination group (skiSE+TLRa), the formulation used to make the DMN patches contained $29 \%(\mathrm{v} / \mathrm{v})$ of SE, $14 \%(\mathrm{v} / \mathrm{v})$ trehalose, $1.25 \%(\mathrm{v} / \mathrm{v})$ polyvinyl alcohol (PVA), along with iPRRSV and the same dose of TLRa as described above. One dose of vaccine was administered in 2 patches and each patch contained 120 microneedles and was $8 \mathrm{~cm}^{2}$ in area (240 microneedles in total). Patches were fabricated as described previously ${ }^{27}$.

\section{Animals and housing}

For this study fifty-four ( $\mathrm{n}=54)$ male three-day-old piglets (Topigs Norsvin Z-line, commercial breed) were purchased from a PRRSV-negative (confirmed with a commercial antibody ELISA) high health status farm (van Beek SPF Varkens B.V., the Netherlands). The piglets received colostrum from the sow and were weaned at 1-2 days of age. After weaning, the piglets were stratified based on their weight and family background (pigs were from 8 different sows) followed by a randomisation to six groups ( $n=9$ for each group). The different groups were housed in separate stables in an isolation unit with HEPA filtered air. Stables were enriched with straw and different toys. 


\section{Experimental design vaccination experiment and sampling}

All the pigs except for the non-vaccinated (NV) group received a prime vaccination at 3-days of age (D0) followed by a booster vaccination (D28) at 4.5-weeks of age (groups are described in Table 1). The NV animals received $1.0 \mathrm{ml}$ of PBS i.m. All i.m vaccines $(1.0 \mathrm{ml})$ were administered in the lateral side of the right hind-leg, whereas the DMNpatches were applied at the medial side of both hind legs. The DMN-patches were removed after 24 hours.

Three weeks after the booster vaccination (D49; 7.5 weeks of age) the animals from groups 1, 2, 3, 5 and 6 received an intranasal challenge with PRRSV $07 \mathrm{~V} 063\left(10^{5} \mathrm{TCID}_{50}\right)$ in PBS (1.0 ml per nostril). The challenge virus was prepared as described previously ${ }^{27}$. At this time point, the pigs from the TLRa group were dissected and lungs were used as reference representing non-PRRSV infected lungs. Three weeks post challenge (D70) the pigs of groups $1,2,3,5$ and 6 were dissected. as previously described ${ }^{27}$.

Serum was sampled at D21, D28 (post-prime), D35, D42, D48 (post-boost), D52, D54, D57, D59, D63, D66 and D70 (post-challenge) to determine antibody response and virus titres. Heparin stabilized blood samples (approximately $15 \mathrm{ml}$ ) were obtained at D21, D42, D56 and D63 for IFN- $\gamma$ ELISpot assay or flow cytometry (FCM).

Table 1: Adjuvant formulations and administration route.

\begin{tabular}{|c|c|c|c|}
\hline \multirow{2}{*}{$\begin{array}{l}\text { Vaccine } \\
\text { formulation }\end{array}$} & \multicolumn{2}{|l|}{ Adjuvant } & \multirow{2}{*}{$\begin{array}{l}\text { Delivery route } \\
\text { and Volume }(\mathrm{ml})\end{array}$} \\
\hline & Immuno-stimulator & O/W emulsion & \\
\hline \multirow[t]{2}{*}{ ISA28 } & - & $\begin{array}{l}\text { Montanide }{ }^{\mathrm{TM}} \\
\text { ISA 28R VG }\end{array}$ & i.m. \\
\hline & & $15 \% \mathrm{v} / \mathrm{v}$ & 1.0 \\
\hline \multirow[t]{2}{*}{ SWE } & - & $\begin{array}{l}\text { Squalene based emulsion (SWE) } \\
42 \% \mathrm{v} / \mathrm{v}\end{array}$ & i.m. \\
\hline & & & 1.0 \\
\hline \multirow[t]{2}{*}{ TLRa } & $\begin{array}{l}\text { 80ụg Pam3Cys } \\
80 \text { ųg R848 }\end{array}$ & - & \\
\hline & $80 u g$ CpG & & 1.0 \\
\hline \multirow[t]{3}{*}{ SWE+TLRa } & 80ug Pam3Cys & Squalene based emulsion (SWE) & i.m. \\
\hline & 80ųg R848 & $42 \% \mathrm{v} / \mathrm{v}$ & \\
\hline & 80ug CpG & & 1.0 \\
\hline \multirow[t]{3}{*}{ skiSE+TLRa } & 80ųg Pam3Cys & Stable emulsion (SE)with squalene & Skin \\
\hline & $80 u g$ R848 & $29 \% \mathrm{v} / \mathrm{v}$ & DMN-patch \\
\hline & $80 u g$ CpG & & na \\
\hline
\end{tabular}

Abbreviations: oil-in-water (O/W emulsion); volume by volume (v/v); Toll-like receptor agonist mixture (TLRa); intramuscular (i.m.); skin vaccination (ski); dissolving microneedle (DMN)-patch; not applicable (na). 


\section{Monitoring of post-vaccination and challenge reaction}

After vaccination the injection site or DMN-patch application area was monitored over 4 days for local effects. For skin vaccination we evaluated redness and swelling of skin, graded from 0 to 3 (no changes, mild, moderate or severe changes) for each hind leg (maximum total score of 6). For the i.m. vaccination we palpated and observed the injection site for increased consistency, redness and local temperature (grade 0-3). Around vaccination and challenge the body temperature and clinical signs were monitored as described before ${ }^{27}$.

\section{PRRSV-specific immune responses}

PRRSV-specific IgG antibodies in serum samples were assessed with an indirect antibody ELISA (Ingezim PRRS 2.0) according to the manufacturer's instruction. A sample-topositive $(S / P)$ ratio of equal or greater than 0.4 was considered positive.

The specific cellular immune response was evaluated with an enzyme-linked immunospot assay (ELISpot assay) kit (Porcine IFN- $\gamma$ ELISpot PLUS (ALP) from Mabtech) ${ }^{40}$ as described before ${ }^{27}$. Next to the ELISpot, we analysed the specific cellular responses against PRRSV in PBMCs by FCM. On D21, D42 and D56 percentages of intracellular TNF or IFN- $\gamma$ staining cells were determined in T-cell subsets and NK-cells. On D56 and D63, unstimulated PBMCs were analysed for relative proportions of T-cell subsets, NK-cells and B-cells within the total PBMC population. For the intracellular staining, PBMC were re-stimulated with PRRSV, as described for the ELISpot. For the last $4 \mathrm{~h}$ of stimulation, we added Brefeldin A (BD Bioscience) to each well to inhibit cytokine release and allow intracellular detection of cytokines. As positive control we added leucocyte activation cocktail (containing ionomycin and phorbol-12-myristate-13-acetate (PMA)) with BD GolgiPlug ${ }^{\mathrm{TM}}$ (BD Biosciences) according to manufacturer's instruction and as negative control we left samples unstimulated with Brefeldin A. Cells were then harvested and the cytokineproduction of T-cell subsets and NK-cells was determined using a 4-step 6-colour staining protocol. Cells were first incubated with the BD Horizon ${ }^{\mathrm{TM}}$ Fixable Viability Stain 450 (FVS450 from BD Biosciences) according to the manufacturer's instructions. The cells were then incubated with directly labelled PE-Cy ${ }^{\mathrm{TM}} 7$ mouse anti-pig CD3E (clone BB23-8E6-8C8 from BD Biosciences), PerCP-Cy ${ }^{\mathrm{TM}} 5.5$ mouse anti-pig CD4a (clone 74-12-4 from BD Biosciences) and Alexa Fluor $647^{\circledR}$ anti-CD8a (clone PG164A, WSU, Pullman, WA, USA) antibodies. Following surface staining, cells were fixed in $4 \%$ paraformaldehyde and after a wash with $0.1 \%$ saponin (Panreac Applichem), cells were incubated with directly labelled Alexa Fluor $647^{\circledR}$ anti-human TNF-a (clone MAb11 from BioLegend), PE mouse anti-pig IFN- $\gamma$ (clone P2G10 from BD Biosciences) in $0.3 \%$ saponin followed by another $0.1 \%$ saponin wash. On D56 and D73 unstimulated PBMCs were stained with the same surface markers as described for the stimulated PBMCs and directly labelled Alexa Fluor $647^{\circledR}$ mouse anti-pig CD21 (clone BB6-11C9.6 from SouthernBiotech) was added as additional surface marker for B-cells. 
PBMCs were analysed on a FACSVERSE ${ }^{\mathrm{TM}}$ (BD Biosciences) using the BD FACSsuite ${ }^{\mathrm{TM}}$ software. The flow cytometry data were analysed with Flowjo ${ }^{\mathrm{TM}}$ software version 10.0. The gating strategy is depicted in the supplementary data (Supp.Fig.3). First doublets and dead cells were excluded and cells were gated for PBMCs. After this cells were classified by expression of the following combinations of surface markers ${ }^{41,42}$ : $T$ cells $\left(C D 3^{+}\right), T$ helper (Th) cells $\left(\mathrm{CD}^{+} \mathrm{CD}^{+}\right)$, Ag-experienced T-cells (Tm) $\left(\mathrm{CD}^{+}{ }^{+} \mathrm{CD} 4^{+} \mathrm{CD} 8 \mathrm{a}^{+}\right)$, cytotoxic T cells (Tcyto) $\left(\mathrm{CD}^{+} \mathrm{CD} 4-\mathrm{CD} 8 \mathrm{a}^{+}\right)$, NK-cells $\left(\mathrm{CD} 3^{-} \mathrm{CD} 8 \mathrm{a}^{+}\right)$and $\mathrm{B}$-cells $\left(\mathrm{CD} 3^{-} \mathrm{CD} 21^{+}\right)$and this classification is used throughout this study. The proportion of the different subpopulations was measured as percentage (relative level) within the live PBMC population. For the Th, Tm, Tcyto and NK cells, we gated the percentage of TNF or IFN- $\gamma$ positive cells in these specific populations.

\section{PRRSV viremia and lung pathology}

Virus titres in serum $\left(\mathrm{TCID}_{50}\right)$ were determined by virus titration as described before ${ }^{27}$. Briefly, porcine alveolar macrophages (PAM) were cultured and ten-fold dilution series of the serum samples (six dilutions for each sample) were added to the PAM, after 3 days the monolayers were stained by immune peroxidase monolayer assay (IPMA) to visualize infection in the cells.

PRRSV-associated lung pathology was assessed by a veterinary pathologist as described previously ${ }^{27}$. Briefly, macroscopic lung lesions were scored to estimate the percentage of affected lung tissue. For histology, hematoxylin and eosin (HE) stained lung tissues (three sections per lung) were scored for the presence of perivascular and peribronchiolar inflammatory infiltrate, and for the alveolar wall infiltrate from 0 (no findings) to 5 (extended manifestation).

\section{Statistical analysis}

GraphPad Prism 8.1.1. was used for statistical analysis. The distribution of the data was investigated with descriptive statistics. Normal distributed data (in vitro cytokines, ELISA, FCM, viremia, body temperature and FCM-TNF,) were analysed with a one-way ANOVA followed by a Tukey's post hoc correction for multiple comparisons. Non-parametric data (ELISpot, skin changes, clinical signs and lung pathology,) were analysed with a Kruskal-Wallis test followed by a post-hoc Dunn's multiple comparisons test. P- values less than 0.05 were considered statistical significant and ${ }^{*} p<0.05,{ }^{* *} p<0.01$, ${ }^{* * *} p<0.001$, ${ }^{* * * *} p<0.0001$ denotes a significant difference of a vaccine formulation compared to the NV animals. Vaccine groups displaying different letters ( $a, b$ and $c)$ show a significant difference between these groups. 


\section{Results}

In vitro cytokine responses after TLR stimulation in neonatal PBMCs

Single TLR agonists (TLR1/2, 7/8 or 9) and TLR agonist combinations (TLR1/2+9 and TLR1/2+7/8+9) were used to stimulate neonatal PBMCs to determine their in vitro potency to elicit cytokine responses. IFN- $\gamma$ and IL12p40 were mainly produced after stimulation of TLR7/8 and 9 and after stimulation with both combinations (TLR1/2+9 and TLR1/2+7/8+9), where high levels of IL-12p40, but lower levels of IFN- $\gamma$ were observed compared to unstimulated control samples (Fig.1 A and B). High levels of IFN-a were only observed after stimulation of TLR9 and the TLR1/2+9 combination. Surprisingly, the response diminished when TLR7/8 was added to the TLR9 stimulation (Fig.1C).

The overall IL-4 response was low but significantly increased for all stimuli, except for stimulation of TLR1/2 (Fig.1D). Significantly elevated levels of IFN- $\gamma$, IL-12p40, IL-4, IL-1 $\beta$, IL-6 and IL-10 were induced when TLR7/8 was present (Fig.1A, B, D-G) compared to the unstimulated control samples. Notably, only TLR7/8-containing stimuli induced significant increases in IL-1 $\beta$, IL- 6 and IL-10. In contrast, the TLR1/2 agonist, Pam3Cys, was the least potent of the three TLRa to induce this panel of cytokines. Elevated levels for IL-8 and TNF were observed with a mixed pattern in all groups, albeit not significant $(\mathrm{Fig} .1 \mathrm{H}$ and I). Overall, the TLR1/2+7/8+9 agonist combination stimulated neonatal PBMC to produce significant levels of IFN- $\gamma$, IL-12p40, IL-4, IL-1 $\beta$, IL- 6 and IL-10 compared to unstimulated control samples and the TLR1/2 agonist showed minimal potential to induce cytokine production compared to the TLR7/8 and 9 agonists. 

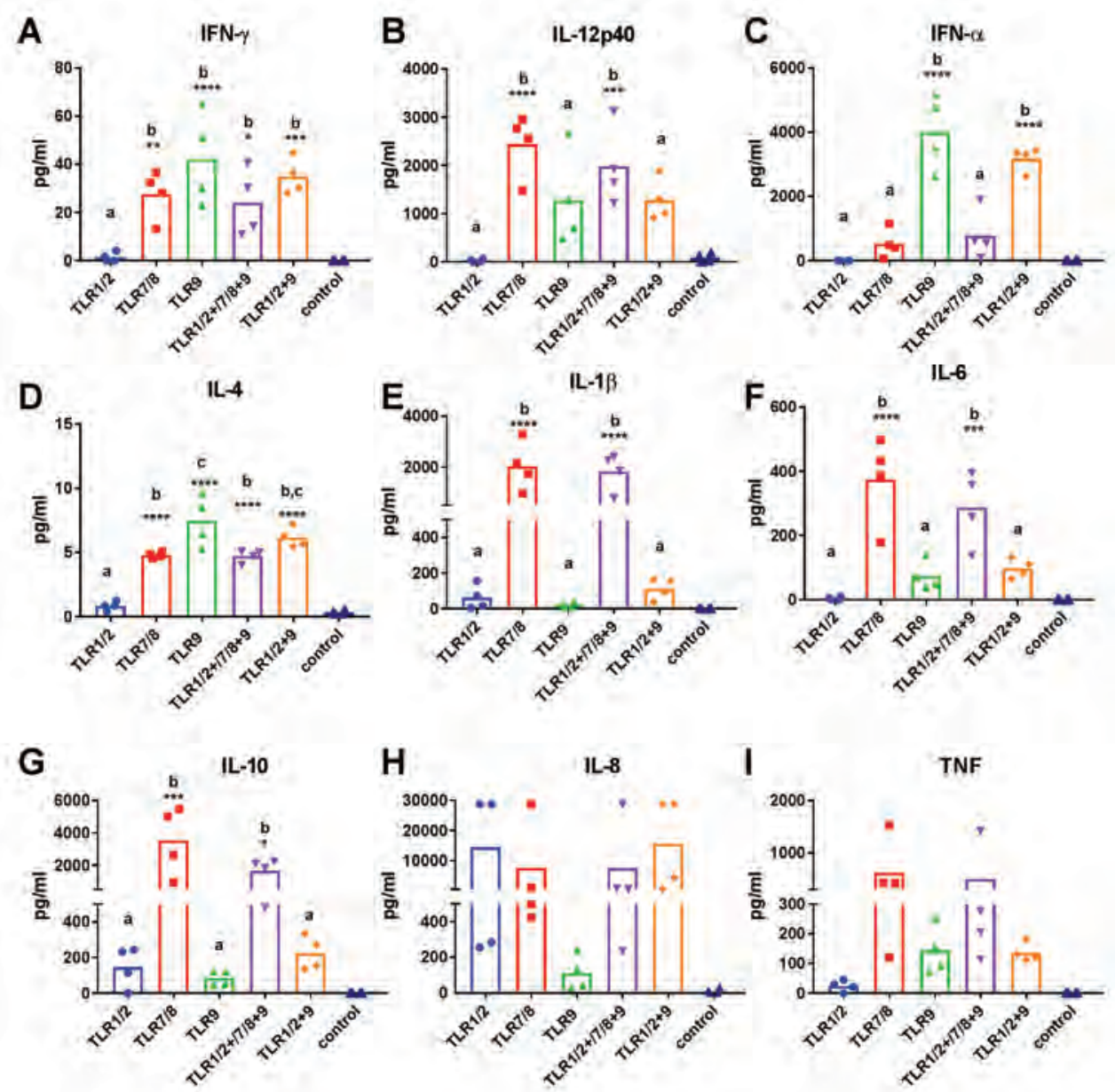

Figure 1: Cytokine response after TLR agonist stimulations in neonatal pigs.

PBMCs of 3-day-old pigs $(n=4)$ were stimulated with different Toll-like receptor agonists (TLR1/2, TLR7/8 or TLR9) and combinations of TLR agonists ( TLR1/2+7/8+9 and TLR1/2+9). Supernatants were tested for (A) IFN- $\gamma$, (B) IL-12p40, (C) IFN- $\alpha$, (D) IL-4, (E) IL-1 $\beta$, (F) IL-6, (G) IL-10, (H) IL-8 and (I) TNF by Luminex multiplex. Each symbol represents one animal and the mean of the group is indicated by the bar. 


\section{Local vaccine responses and challenge reaction}

After prime and booster vaccination, none of the tested vaccine formulations induced any systemic adverse effects, such as raise in body temperature, loss of appetite and activity, or reduced weight gain (data not shown). A mild local skin reaction (grade 1) was observed in most of the animals after prime DMN-patch application, characterized by a variable local redness (Supp.Fig.1A and D). However, after the booster vaccination (4.5 weeks of age) we observed in nearly all animals significantly more skin reaction (grade 2 and 3) (Supp.Fig.1B and D) than after prime vaccination. This moderate to severe skin reaction was still visible 4 days after vaccination (Supp.Fig.1C), but was completely disappeared two weeks after booster vaccination. No local reaction was observed after i.m. administration, as observed by palpation or macroscopic muscle changes during necropsy.

After challenge (from 3-11 days post infection (dpi)) there was a raise in body temperature in all the groups. This increased body temperature was combined with PRRSV-related clinical signs. The main observed signs were loss of appetite, reduced liveliness and a few animals were coughing or showed skin changes of the ears. There was no significant difference between the various vaccine groups, or between the vaccine groups and NV group with regard to body temperature or PRRSV-related clinical symptoms (Supp.Fig.2A and B). At the time of the necropsy (21 dpi), none of the pigs displayed any clinical symptoms. There were no significant differences in weight or weight gain between the groups after challenge (results not shown).

\section{PRRSV-specific humoral immune response}

PRRSV-specific IgG antibodies were analysed in the serum to investigate the specific humoral immune response. Two weeks after booster vaccination (D42) only the ISA28 group showed a detectable PRRSV antibody response ( $S / P>0.4)$ in 4 of the 9 animals (Fig.2A), while none of the animals in the other vaccine groups developed a detectable specific antibody before challenge.

From ten days after challenge (D59 or $10 \mathrm{dpi}$ ) onwards all vaccinated groups developed a PRRSV-specific antibody response, which was significantly higher on D59 compared to the NV animals. The ISA28 group showed a significantly higher specific antibody response compared to the other vaccine groups. From D63 (14 dpi) also the NV animals showed a specific antibody response (Fig.2A).

The area under the curve (AUC) value, representing the total antibody response after challenge (D52-D70) (Fig. 2B), was significantly higher for all vaccinated groups compared to the NV group. However, a significant higher specific antibody response was found for ISA28 as compared to the other adjuvant groups. 
A

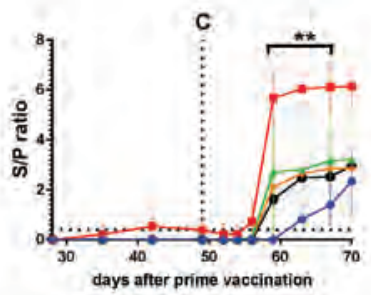

B

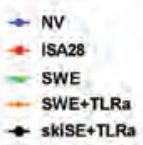

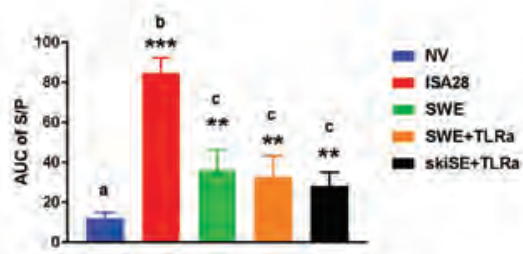

Figure 2: Kinetics of PRRSV-specific antibody response after booster vaccination and challenge.

PRRSV antibody titres were measured with ELISA. (A) PRRSV-specific lgG titres after booster vaccination (D28), in non-vaccinated (NV) or iPRRSV vaccinated animals using different adjuvants (ISA29, SWE, TLRa, SWE+TLRa) and skin vaccination (skiSE+TLRa); each data point represents the mean of 9 animals $\pm S D$; the dotted line marks the limit of detection for the S/P ratio; $C$ indicates challenge at D49; (B) Area under the curve (AUC) for specific antibody production after challenge.

\section{PRRSV-specific cellular immune response (ELISpot)}

The cellular immune response was evaluated by the number of IFN- $\gamma$ secreting cell (SCs) in the PBMCs after re-stimulation with the challenge strain. There was no detectable increase in the number of IFN- $\gamma$ SCs three weeks after prime vaccination (D21) in any of the measured groups (NV, ISA28 and SWE+TLRa) (results not shown).

Two weeks after booster vaccination (D42) the SWE group showed a significant increase in antigen-specific IFN- $\gamma \mathrm{SCs}$. This antigen-specific cellular response was lower (not significant) in the ISA28 group, but absent in the other groups (Fig.3A). One week after challenge (D56) all vaccine groups and the NV group had large numbers of antigenspecific IFN- $\gamma \mathrm{SC}$, however there was no significant difference between the NV group and the vaccine groups (Fig.3B).

\section{Lymphocyte responses measured by flow cytometry}

We also evaluated the cellular immune response after vaccination and challenge by the percentage of positive IFN- $\gamma$ or TNF staining cells in the different T-cell subsets (Th, Tm, Tcyto) and NK-cells within in vitro re-stimulated PBMCs. Three weeks after the prime vaccination (D21) we detected no significant intracellular staining for TNF and IFN- $\gamma$ after PRRSV stimulation in the different T-cell subsets and NK-cells in any of the measured groups (NV, ISA28 and SWE+TLRa) (results not shown). Two weeks after booster vaccination (D42) a significant increase in the percentage of IFN- $\gamma$ positive cells after PRRSV stimulation in all T-cell subsets and NK-cells was observed in the ISA28 and SWE groups compared to NV animals. No response was observed in the other groups (Fig 4A). The overall percentage of cells with TNF staining (average $<0.05 \%$ ) was significantly lower than for the IFN- $\gamma$ staining (average 2.0-5.0\%). Only the Th and Tm subsets in PBMCs of pigs from the ISA28 
A

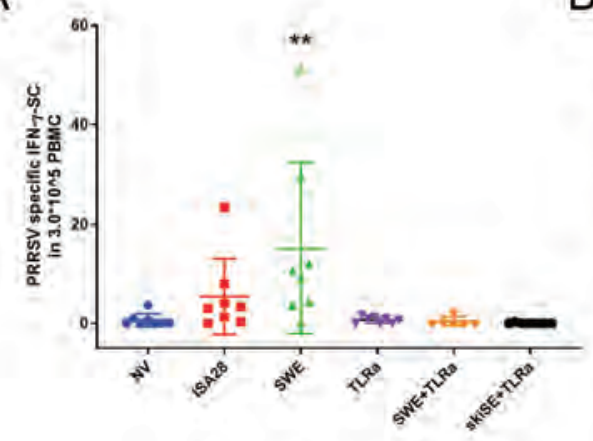

B

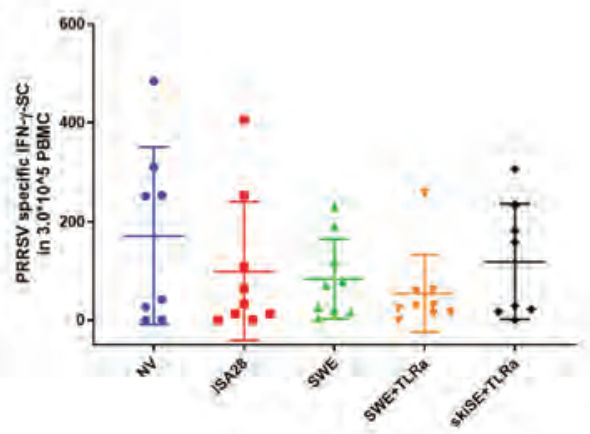

Figure 3: PRRSV-specific T-cell IFN- $\gamma$ response after booster vaccination and challenge (ELISpot).

PBMCs were stimulated with PBS (background correction) or PRRSV 07V063.(A) Number of PRRSVspecific IFN- $\gamma$ secreting cells (SC), in non-vaccinated (NV) or iPRRSV vaccinated animals using different adjuvants (ISA29, SWE, TLRa and SWE+TLRa) and skin vaccination (skiSE+TLRa); (A) two weeks after booster vaccination (D42) and (B) 7 days after challenge (D56); Each symbol represents one animal (mean of triplicate) and the median of the group set $(n=8)$ is indicated by the bar.

and SWE group showed a significant increase in the percentage of TNF positive cells compared to the NV group (Supp. Fig. 4A).

Seven days after challenge (D56) all of the vaccinated animals showed a significantly lower percentage of IFN- $\gamma$ positive cells after PRRSV stimulation in the Th, Tm and Tcyto subsets compared to the NV animals (Fig.4B). This reduction was not significant for the NK-cells. No significant intracellular TNF responses were observed in any of the groups at seven days after challenge. (Supp. Fig.4B).

We also analysed the relative percentages of the different T-cell subsets ( $\mathrm{Tm}$ and Tcyto), NK-cell and B-cells within unstimulated PBMCs 7 and 13 days after challenge (Fig.4C). The ISA28 group was the only group that showed a significant increase in the relative percentage of B-cells between 7 and 13 days after challenge (Fig.4C). The relative percentage of Tm cells significantly increased between 7 and 13 days after challenge in the ISA28, SWE and SWE+TLRa groups. Tcyto cells significantly increased in the ISA28 and SWE groups. None of the vaccine groups showed a significant increase in the relative percentage of NK-cells within the PBMCs between 7 and 13 days after challenge. 

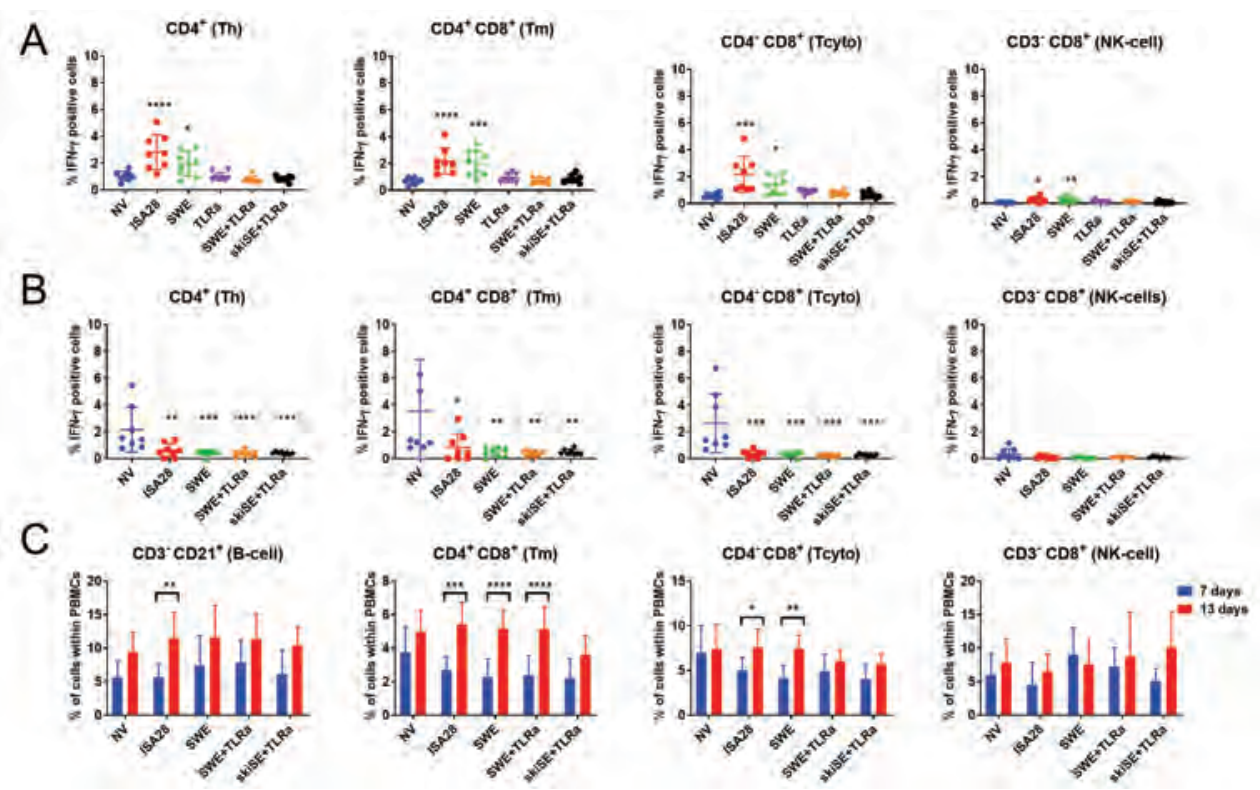

Figure 4: PRRSV-specific intracellular IFN- $\gamma$ responses and lymphocyte subsets (flow cytometry).

PRRSV-specific IFN- $\gamma$ response after in vitro re-stimulation of PBMCs in non-vaccinated (NV) or iPRRSV vaccinated animals using different adjuvants (ISA29, SWE, TLRa and SWE+TLRa) and skin vaccination (skiSE+TLRa). The percentage of positive IFN- $\gamma$ cells within different T-cell subsets (CD4+ (Th), CD4+CD8+ (Tm) CD4-CD8+ (Tcyto)), and NK-cells (CD3-CD8+) were analysed (A) 2 weeks after booster vaccination (D42) and (B) one week after challenge (D56). Each symbol represents one animal and the mean of the group set $(n=8)$ is indicated by the bar; $(C)$ the relative proportion of B-cells (CD3-CD21+), T-cell subsets and NK-cells were determined within the live PBMC population 7 (D56) and 13 (D62) days after challenge; ${ }^{*} p<0.05,{ }^{* *} p<0.01,{ }^{* * *} p<0.001,{ }^{* * * *} p<0.0001$ denotes a significant difference of the vaccine groups compared to the NV control $(A+B)$ or a significant difference between 7 and 13 days after challenge (C).

\section{PRRSV viremia}

Before challenge we detected no PRRSV in the serum as measured by virus titration (Fig.5A). The TLRa group was not challenged with PRRSV and was used as reference for non-PRRSV infected lungs. At D52 (3 dpi) PRRSV was detected in the serum of $88 \%$ of the animals and at $5 \mathrm{dpi}$ all challenged animals were viremic. From $10 \mathrm{dpi}$ onwards the virus titre declined in all the groups. None of the vaccinated groups showed a reduced viremia compared to the NV group as measured on different time-points after challenge or determined as AUC (Fig.5A and B). At the end of the study $21 \mathrm{dpi}$ (D70) 95\% of the animals were still viremic. 
A

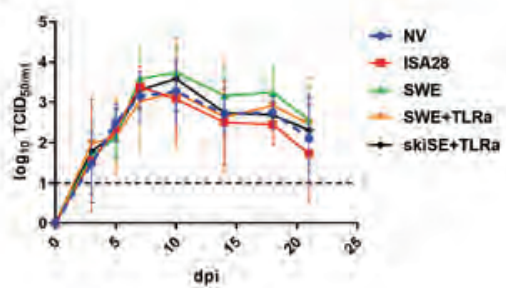

B

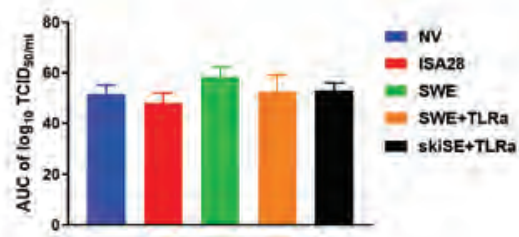

Figure 5: Clearance of PRRSV in serum after challenge.

Virus titres in serum $(\log 10 \mathrm{TCID} 50 / \mathrm{ml})$ were determined by virus titration at different time-points after challenge (days post infection (dpi)). (A) serum virus titres in non-vaccinated (NV) or iPRRSV vaccinated animals using different adjuvants (ISA29, SWE and SWE+TLRa) and skin vaccination (skiSE+TLRa). Each data point represents the average of 9 animals \pm S.D. The dotted line marks the detection limit for virus titration; (B) Area under the curve (AUC) calculated for the whole group with the S.D.

\section{PRRSV induced lung pathology}

The lungs of infected pigs showed mild to moderate macroscopic pathological changes 21 days after challenge in all groups, but there were no significant differences between the NV animals and vaccinated animals (Supp.Fig.5A). No macroscopic lesions were observed in the unchallenged TLRa group. Individual lesion extension ranged from $0 \%$ to $15 \%$ of affected lung surface. The macroscopic changes were mainly observed in the cranial and the middle lung lobe and were characterized by multifocal, irregular, slightly sunken red to tan areas.

There was no significant difference in severity of the lung histopathology between the vaccine groups and NV groups (Supp.Fig.5B, C and D). The lungs of the unchallenged pigs from the TLRa group displayed no significant changes related to PRRSV-infection (Supp.Fig.5E). The lungs of all PRRSV challenged pigs showed mild to moderate interstitial pneumonia characterized by a mononuclear infiltrate of mainly macrophages and lymphocytes in the alveolar septa and around the blood vessels and bronchi and bronchioles. Dispersed alveolar lumina were occluded by the expanded alveolar walls often combined with a similar mononuclear infiltrate occasionally admixed with cellular debris (Supp.Fig.5F and G). 


\section{Discussion}

Efficacy of inactivated and subunit vaccines strongly depends on the immuno-stimulating properties of the selected adjuvant in combination with the specific antigen and the age of the vaccine recipient ${ }^{43}$. Porcine neonates can be vaccinated effectively as studies with inactivated vaccines 33,44 showed specific cellular and humoral immune responses combined with partial or full protection after challenge, although these studies used different adjuvants and antigens. As the immunogenic potential of the iPRRSV is minimal, even in studies with adult pigs ${ }^{5,6}$, there is a strong immuno-stimulator needed for neonatal vaccines. Here, we investigated if vaccinated neonatal pigs developed a protective immune response with the weak iPRRSV antigen using specific adjuvants and different delivery routes. Porcine in vitro studies have shown that neonates can elicit an adult-like DC responses after effective stimulation with TLR1/2, 7/8 and 9 agonists ${ }^{26,45}$, potentially contributing to increased antigen-presentation and subsequent differentiation of T-cells ${ }^{46}$ and thereby increasing vaccine efficacy. A recent porcine study has used these TLR1/2, $7 / 8$ and 9 agonists in a mixture in combined with O/W emulsion (SWE) where this adjuvant formulation induced Th1 and innate immune response, although in adult pigs and with a different antigen ${ }^{28}$.

PBMC stimulation with the TLRa mixture without PRRSV-antigen induced significant levels of the Th1 directed cytokines IFN- $\gamma$ and IL12p40, where we consider that IL12p40 reflects the IL12p35/IL-12 production ${ }^{24}$. This response was accompanied by increased levels of Th2 directed IL-4 and pro-inflammatory cytokines like IL-1 $\beta$ and IL-6. When using TLR7/8 stimulation, but also the TLRa mixture, high levels of IL-10 were observed similar to what has been described after stimulation of human cord blood with TLR7/8 agonists ${ }^{13}$. Where they suggest that this IL-10 response could contribute to B-cell and antibody responses ${ }^{47}$ and could potentially avoid harmful inflammatory responses. Interestingly, the type I IFN-a cytokine response, which was measured after stimulation with a single TLR9 or TLR1/2+9 agonist, was abrogated when the TLR7/8 agonist was added to TLR9 containing stimuli, suggesting that this combination is less effective in inducing type I IFN responses. A similar decrease of IFN-a production when combining TLR7/8 and 9 agonists was observed, when using human PBMCs from adults, but not in cord blood and it was speculated that both TLR agonists would compete for endosomal signalling ${ }^{48}$. Despite the limited IFN-a response, we anticipated that the induced cytokine responses would contribute to a Th1 polarizing vaccine response in neonates as shown in adult pigs 28. Therefore, we selected the TLR $1 / 2+7 / 8+9$ agonist mixture as immuno-stimulator for our neonatal study with iPRRSV. We used PRRSV-naive piglets, to allow us to distinguish adjuvant-related effects without interference from maternal antibodies.

In the vaccine study, neonatal pigs developed a detectable specific immune response after boost vaccination in the O/W emulsion groups: ISA28 (humoral and cellular) and SWE (only cellular), but not in the vaccines containing the TLRa mixture. Surprisingly, the SWE induced IFN-y T-cell response was not initiated when TLRa were co-administered 
as observed in both FCM and ELISpot. This suggests that administration of the TLRa modulated the development of PRRSV-specific cellular immune responses, despite the fact that we anticipated that the TLRa would enhance the immune response. A similar observation was made in mice using a peptide-based vaccine where a water-in-oil (W/O) emulsion abrogated the CpG (TLR9a) induced immune response, while a squalene-based $\mathrm{O} / \mathrm{W}$ emulsion enhanced the cellular immune response ${ }^{49}$. We speculate that a TLRa induced tolerance of the innate immune system after prime vaccination, or a downregulation of TLR-expression could mitigate the booster vaccination with the TLRa mixture. Also in mouse macrophages, tolerance was induced along with a reduced expression of TLR7 and 9 upon the use of a TLR7 and 9 agonist-supplemented vaccine ${ }^{50}$. In addition, the total cytokine response and its profile induced by SWE+TLRa could inhibit the immune response similar to a neonate non-human primate study in which co-administration of a TLR5 agonist with a TLR7/8 agonist did not enhance the vaccine protection, but induced elevated C-reactive protein (CRP) levels after vaccination ${ }^{51}$. Finally, the limited type I IFN response in vitro when using the TLRa mixture could contribute to less effective induction of the adaptive immune response ${ }^{52}$ in vaccine formulations containing TLRa.

Only the ISA28 group induced a specific humoral immune response after booster vaccination, where only post-challenge a memory-antibody response was observed in the SWE, SWE+TLRa and the skiSE+TLRa groups. The absence of significant differences between the vaccine groups with a memory-antibody response indicates that the humoral immune response was not influenced by TLRa in a positive or negative manner. In general, $\mathrm{O} / \mathrm{W}$ emulsion induce a strong but short-term immune response, especially humoral ${ }^{53,54}$, but as well cellular as observed for ISA28 in pigs ${ }^{27,55}$ and SWE in pigs ${ }^{28}$ and mice ${ }^{56}$. Presumably, the B-cell response after vaccination in the SWE, SWE+TLRa and the skiSE+TLRa groups was restricted to a memory B-cell response, without detectable production of PRRSV-specific IgG by plasma cells. This could be related to the neonatal immune status, as it was shown in humans that neonates are more primed for the induction of memory B-cell formation rather than plasma cell differentiation ${ }^{14}$. Only ISA28 induced PRRSV-specific plasma cells after vaccination in neonates, supported by a significantly increased B-cell proportion after challenge, which was not observed in other vaccine groups. Differences in emulsion composition, e.g. in mineral oil or in the surfactants, or in antigen-adjuvant interaction could be related to this humoral disparity between ISA28 and SWE in neonates.

With respect to the induction of cellular immunity after challenge, we anticipated an increased IFN- $\gamma$ T-cell response in the vaccinated groups compared to the NV group. However, 7 days after challenge no significant changes between vaccinated and NV animals for IFN- SCS (ELISpot) were observed and even a reduced percentage of IFN- $\gamma$ positive-specific T-cells compared to the NV animals was observed in the FCM. This could be related to the time-point of analysis as other studies ${ }^{32,57}$ detected a maximal IFN- $\gamma$ response in PRSSV vaccinated animals more than 3 weeks after challenge. As well, we 
suggest that the T-cells of the vaccinated animals have become anergic to further in vitro stimulation. Alternatively, in vaccinated animals a more effective homing to peripheral tissues of activated antigen-specific T-cells might occur, thereby reducing the number of IFN- $\gamma$ producing cells within the PBMC population. These results also confirm that NV animals were able to develop a distinct specific cellular immune response within one week after challenge with the PRRSV strain 07V063. This is quicker than the two or three weeks that has been described in other studies $4,58,59$

The delivery route is also an important factor contributing to the induction of an effective vaccination response. We showed a lack of immunogenicity and efficacy after i.m. as well as after skin-based vaccination. However, the exacerbated skin reaction after booster vaccination with DMN-patch could indicate a delayed type hypersensitivity (DTH) reaction induced by a strong local Th1 response ${ }^{60}$ suggesting that a T-cell response was induced by skin immunisation, however these responses were restricted to the skin and/ or non-peripheral blood components. Despite the induction of a DTH reaction in the skin, the clinical signs and lung pathology after challenge were not intensified in these or any of the vaccinated groups ${ }^{61}$, which makes a PRRSV-related reaction less likely. In our previous vaccine study ${ }^{27}$ using 6-week-old pigs with similar DMN-patches we did not observe this exacerbated skin response after booster vaccination. However that study did not test the delivery of a PRRSV vaccine with a combination of a SWE and a mixture of TLR agonists and moreover used a shorter DMN (500 mm in adults, compared to $600 \mathrm{~mm}$ in neonates).

None of the vaccines used in this study showed any efficacy in vivo as measured by reduction of viremia, mitigation of lung pathology or decreased clinical signs after PRRSV challenge. However, the measured immune responses provided insights for future porcine neonatal research. Based on the PBMC stimulation and our in vivo results, we hypothesize that an O/W emulsion with only a TLR7/8 agonist could be more effective in neonates, as neonatal studies have shown that TLR7/8 agonist in mice and non-human primates ${ }^{51,62,63}$ enhanced the Th1 directed response and B-cell activation. Also, a next step would be to investigate the immune responses in the skin and draining lymph node shortly after vaccination to study in more detail, how the TLRa contribute to the development of the immune response in neonatal pigs and the DTH reaction after skin booster vaccination.

\section{Conclusion}

The O/W emulsions ISA28 or SWE combined with iPRRSV induced a vaccine-specific immune response after booster vaccination in PRRSV-naïve neonatal pigs, this specific immune response was not observed in the vaccines containing TLRa. However, none of the vaccines were able to reduce the viremia and lung pathology when prime vaccination was applied at three-days of age. Further research focussed on the early immune response is needed to elucidate the potential of TLRa in vaccines for neonatal pigs. 


\section{Conflict of interest statement}

Anne Moore is an inventor of patents that have been or may be licensed to companies developing microneedle-based products. This potential competing interest has been disclosed and is being managed by University College Cork. Dennis McDaid is Chief Operating Officer and current director and owns stock in Xeolas Pharmaceuticals Limited. Damien Collins is a former employee of Xeolas Pharmaceuticals Limited and have no financial or other competing interests. The other authors have no competing interest to declare.

\section{Acknowledgments}

The authors thank Prof.dr. Artur Summerfield (Bern, Switzerland) for his advice regarding adjuvant selection as partner of the SAPHIR Horizon 2020 Programme. Rene Achterberg and Lisette Ruuls are acknowledged for their technical assistance. The animal caretakers are thanked for collection of all samples and providing optimal housing conditions for the pigs used in this experiment. Conor O'Mahony at the Tyndall National Institute, UCC is acknowledged for supplying PDMS moulds. This project has received funding from the European Union's Horizon 2020 Programme for research, technological development and demonstration under the Grant Agreement $n^{\circ} 633184$. This publication reflects the views only of the author, and not the European Commission (EC). The EC is not liable for any use that may be made of the information contained herein. 


\section{Supplementary data}

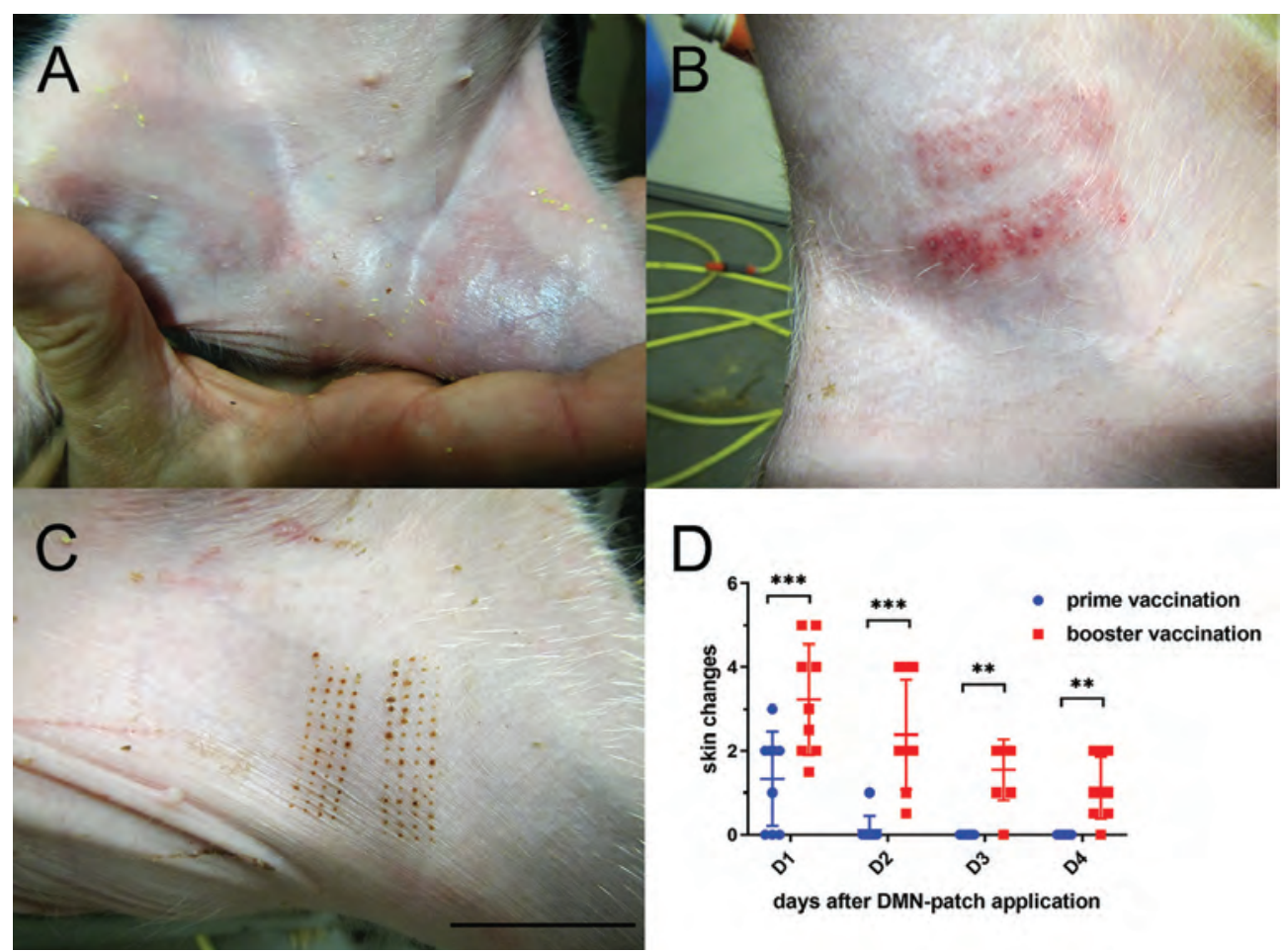

Figure 1: Skin reaction after dissolving microneedle (DMN) patch application with skiSE+TLRa.

Examples of grading of skin changes based on redness and swelling of skin on the medial side of hind-leg. (A) minimal skin changes (grade 1) one day (D1) after booster vaccination; (B) severe skin changes (grade 3) on D1 after booster vaccinations; (C) skin changes 4 days (D4) after booster vaccination; scale bar length $=4 \mathrm{~cm}$; (D) Skin reaction overview from D1 till D4 after prime vaccination (blue symbols) and after booster vaccination (red symbols), both hind legs were graded (maximum total score of 6). Each symbol represents one animal and the bar indicates the median of the group $(n=9)$. 
A

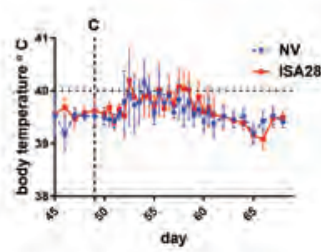

B

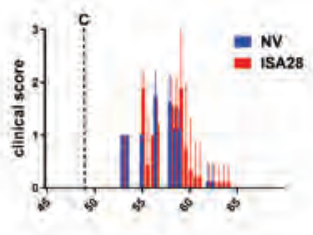

day
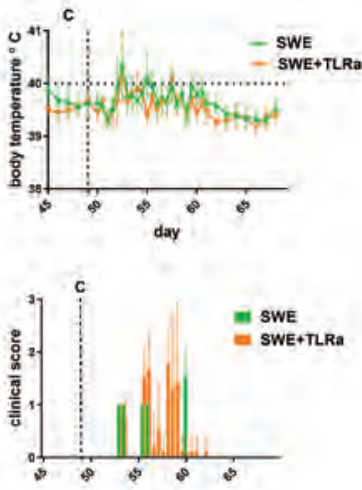

day
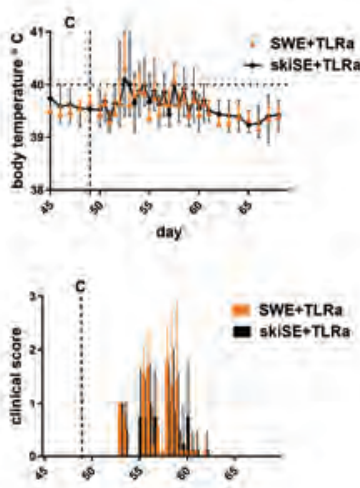

day

Figure 2: PRRSV challenge: body temperature and clinical score.

(A) The average body temperature and (B) average clinical score after PRRSV challenge in nonvaccinated (NV) or iPRRSV vaccinated animals using different adjuvants (ISA29, SWE, TLRa and SWE+TLRa) and skin vaccination (skiSE+TLRa); each symbol represents the median (clinical score) or mean (body temperature of one group ( $n=9) \pm S$.E (clinical score) or S.D. (body weight); $C=$ challenge at D49.

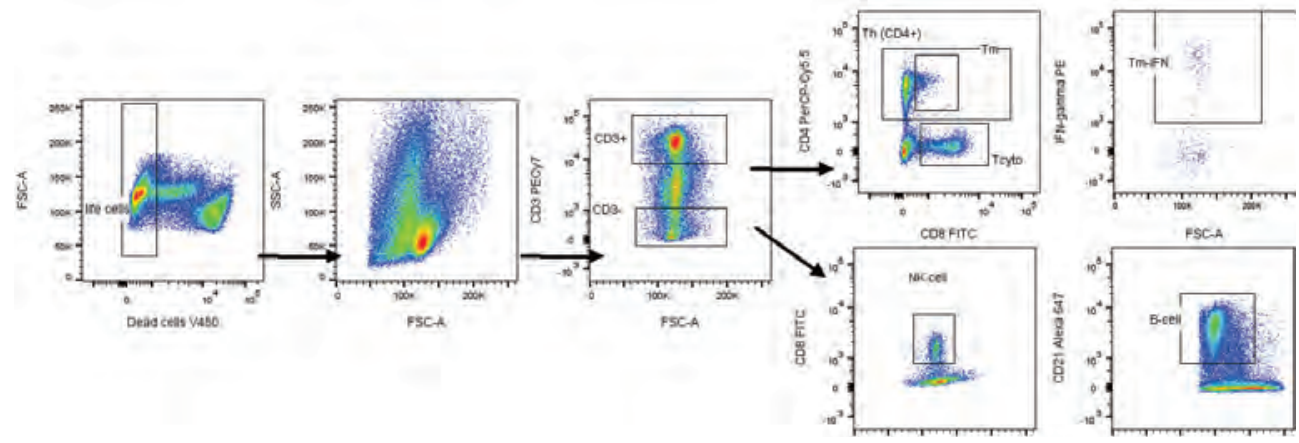

Figure 3: Flow cytometry gating strategy T-cell subsets, B-cells and NK-cells.

Dead cells and doublets (not shown) were excluded and PBMCs were gated for CD3+ (T-cells) and CD3- (NK-cells and B-cells). CD3+ cell were further specified with the surface-markers CD4 and CD8a: Thelper (Th) cells (CD4+ CD8+/-), Ag-experienced Th cells (Tm) (CD4+CD8a+) and cytotoxic T cells (Tcyto) (CD4-CD8a+). CD3- cells were stained with surface markers CD8a for NK-cells (CD3-CD8a+) and CD21 for B-cells (CD3-CD21+). Within the different T-cell subset and NK-cells the percentage of IFN- $\gamma$ positive cells or TNF positive cells (not shown) was determined on D21, D42 and D56. For the T-cell subsets, NK-cells and B-cells we determined the relative percentage of these populations within the live PBMC population on D56 and D62. 
A

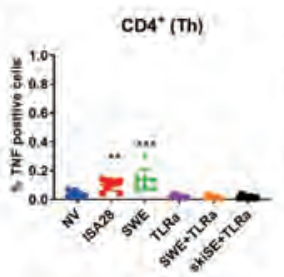

B

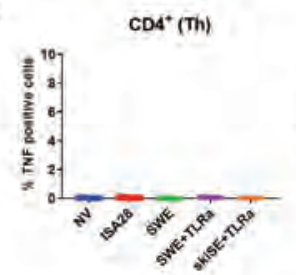

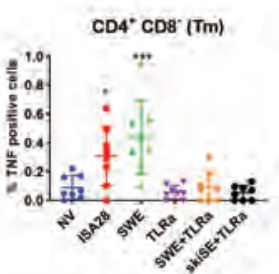

$\mathrm{CD}^{*} \mathrm{CDB}^{*}(\mathrm{Tm})$

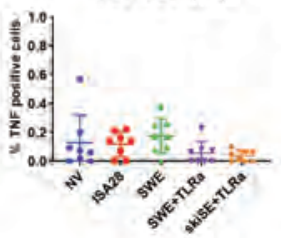

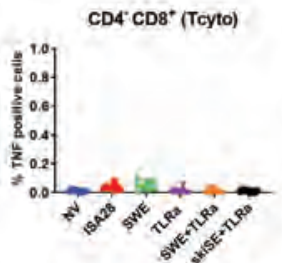

$\mathrm{CD}^{*} \mathrm{CDB}^{*}$ (Tcyto)

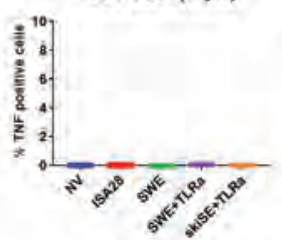

CD3 $\mathrm{CDB}^{*}$ (NK-cells)

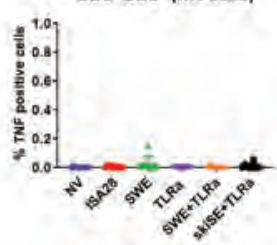

CD3' $\mathrm{CD}^{*}$ (NK-cells)

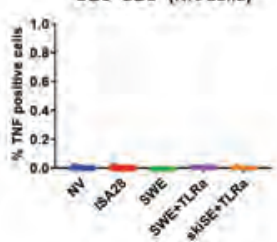

Figure 4: PRRSV specific intracellular TNF responses after booster vaccination and challenge (flow cytometry).

PRRSV-specific TNF response after in vitro re-stimulation of PBMCs in non-vaccinated (NV) or iPRRSV vaccinated animals using different adjuvants (ISA29, SWE, TLRa and SWE+TLRa) and skin vaccination (skiSE+TLRa). The percentage of positive TNF cells within different T-cell subsets $\left(\mathrm{CD}^{+}(\mathrm{Th})\right.$, $\mathrm{CD}^{+} \mathrm{CD}^{+}(\mathrm{Tm}) \mathrm{CD}^{-} \mathrm{CD} 8^{+}$(Tcyto)), and NK-cells (CD3 $\left.{ }^{-} \mathrm{CD} 8^{+}\right)$were analysed (A) 2 weeks after booster vaccination (D42) and (B) one week after challenge (D56). Each symbol represents one animal and the mean of the group set $(n=8)$ is indicated by the bar. 

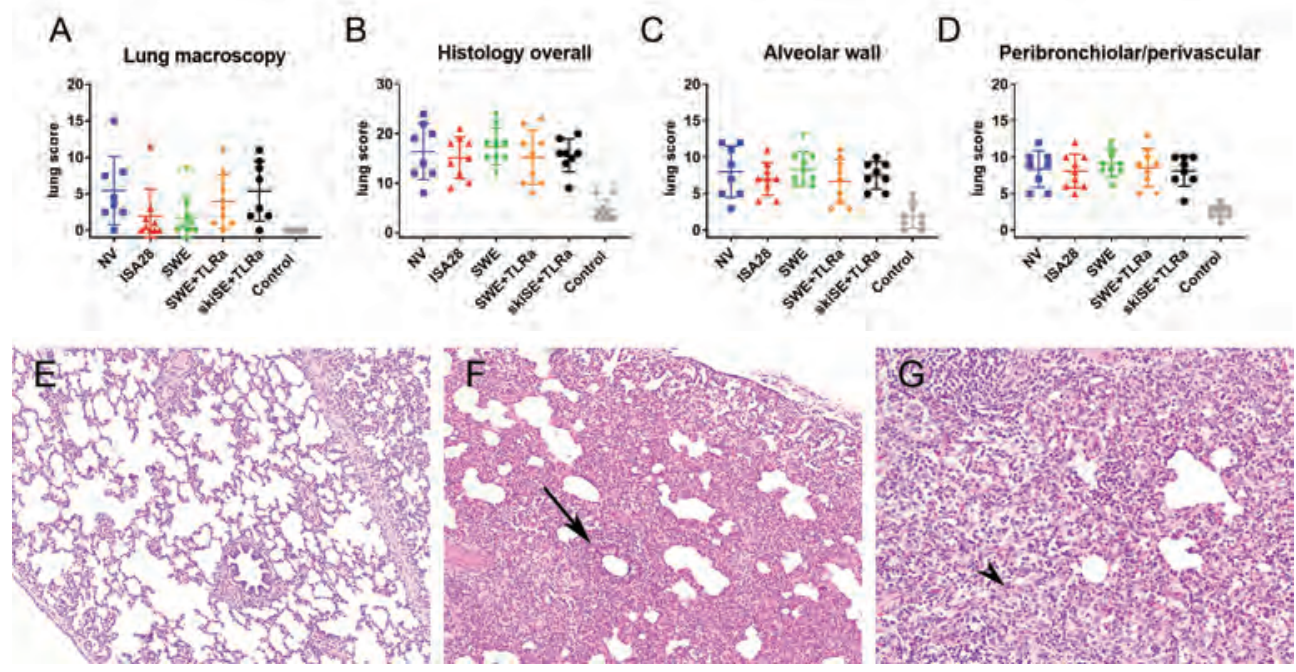

Figure 5: Lung pathology 21 days after challenge.

Macroscopic lung changes and histopathology of lung lesions after PRRSV challenge in nonvaccinated (NV) or iPRRSV vaccinated animals using different adjuvants (ISA29, SWE and SWE+TLRa) and skin vaccination (skiSE+TLRa). The lungs of the unchallenged TLRa group were used as reference (control); (A) The extension of macroscopic lung lesions was calculated as proportion of total lung area; (B) Histologic lesions evaluated in the left cranial, medial and caudal lung lobe for infiltrate of mononuclear inflammatory cells in alveolar walls (score 0-5 for each lobe), (C) and around blood vessels, bronchi and bronchioli (score $0-5$ for each lobe) (D) combined to an overall histology score (score 0-30); Each symbol represents one animal and the median is shown for the data set $(n=8),(E)$ Normal lung of unchallenged control pig (TLRa) (Haematoxylin and Eosin (HE) stain; objective 10x); (F) interstitial pneumonia 21 days after PRRSV infection with a significant mononuclear infiltrate of mainly macrophages and lymphocytes around blood vessels and bronchi and bronchiole (arrow) (objective 10x); (G) alveolar walls are thickened by the same type of inflammatory cells (arrowhead) (objective 20x). 


\section{References}

1 Holtkamp, D. J.et al. Assessment of the economic impact of porcine reproductive and respiratory syndrome virus on United States pork producers. Journal of Swine Health and Production 21, 72-84 (2013).

2 Nathues, H. et al. Cost of porcine reproductive and respiratory syndrome virus at individual farm level - An economic disease model. Preventive veterinary medicine 142, 16-29, doi:10.1016/j.prevetmed.2017.04.006 (2017).

3 Renukaradhya, G. J., Meng, X. J., Calvert, J. G., Roof, M. \& Lager, K. M. Inactivated and subunit vaccines against porcine reproductive and respiratory syndrome: Current status and future direction. Vaccine 33, 3065-3072, doi:10.1016/j.vaccine.2015.04.102 (2015).

4 Zuckermann, F. A. et al. Assessment of the efficacy of commercial porcine reproductive and respiratory syndrome virus (PRRSV) vaccines based on measurement of serologic response, frequency of gammaIFN-producing cells and virological parameters of protection upon challenge. Veterinary microbiology 123, 69-85, doi:10.1016/j.vetmic.2007.02.009 (2007).

5 Geldhof, M. F. et al. Comparison of the efficacy of autogenous inactivated Porcine Reproductive and Respiratory Syndrome Virus (PRRSV) vaccines with that of commercial vaccines against homologous and heterologous challenges. BMC veterinary research 8, 182, doi:10.1186/1746-6148-8-182 (2012).

6 Vanhee, M., Delputte, P. L., Delrue, I., Geldhof, M. F. \& Nauwynck, H. J. Development of an experimental inactivated PRRSV vaccine that induces virus-neutralizing antibodies. Veterinary research 40, 63, doi:10.1051/vetres/2009046 (2009).

7 Charerntantanakul, W. Adjuvants for porcine reproductive and respiratory syndrome virus vaccines. Veterinary immunology and immunopathology 129, 1-13, doi:10.1016/j.vetimm.2008.12.018 (2009).

8 Balasch, M., Fort, M., Taylor, L. P. \& Calvert, J. G. Vaccination of 1-day-old pigs with a porcine reproductive and respiratory syndrome virus (PRRSV) modified live attenuated virus vaccine is able to overcome maternal immunity. Porcine health management 4, 25, doi:10.1186/s40813-018-0101-x (2018).

9 Jeong, J. et al. Vaccination with a porcine reproductive and respiratory syndrome virus vaccine at 1-dayold improved growth performance of piglets under field conditions. Veterinary microbiology 214, 113-124, doi:10.1016/j.vetmic.2017.12.023 (2018).

10 Kumar, S. K. \& Bhat, B. V. Distinct mechanisms of the newborn innate immunity. Immunol Lett 173, 42-54, doi:10.1016/j.imlet.2016.03.009 (2016).

11 Levy, O. Innate immunity of the newborn: basic mechanisms and clinical correlates. Nature reviews. Immunology 7, 379-390, doi:10.1038/nri2075 (2007).

12 Saso, A. \& Kampmann, B. Vaccine responses in newborns. Seminars in immunopathology 39, 627-642, doi:10.1007/s00281-017-0654-9 (2017).

13 Kollmann, T. R. et al. Neonatal innate TLR-mediated responses are distinct from those of adults. Journal of immunology 183, 7150-7160, doi:10.4049/jimmunol.0901481 (2009).

14 Siegrist, C. A. \& Aspinall, R. B-cell responses to vaccination at the extremes of age. Nature reviews. Immunology 9, 185-194, doi:10.1038/nri2508 (2009).

15 Poonsuk, K. \& Zimmerman, J. Historical and contemporary aspects of maternal immunity in swine. Animal health research reviews, 1-15, doi:10.1017/s1466252317000123 (2017). 
16 Siegrist, C. A. Mechanisms by which maternal antibodies influence infant vaccine responses: review of hypotheses and definition of main determinants. Vaccine 21, 3406-3412 (2003).

17 Chappuis, G. Neonatal immunity and immunisation in early age: lessons from veterinary medicine. Vaccine 16, 1468-1472 (1998).

18 Zinkernagel, R. M. On natural and artificial vaccinations. Annual review of immunology 21, 515-546, doi:10.1146/annurev.immunol.21.120601.141045 (2003).

19 Kollmann, T. R. \& Marchant, A. Towards Predicting Protective Vaccine Responses in the Very Young. Trends in immunology 37, 523-534, doi:10.1016/j.it.2016.05.005 (2016).

20 Mohr, E. \& Siegrist, C. A. Vaccination in early life: standing up to the challenges. Current opinion in immunology 41, 1-8, doi:10.1016/j.coi.2016.04.004 (2016).

21 de Brito, C. A., Goldoni, A. L. \& Sato, M. N. Immune adjuvants in early life: targeting the innate immune system to overcome impaired adaptive response. Immunotherapy 1, 883-895, doi:10.2217/imt.09.38 (2009).

22 Morris, M. C. \& Surendran, N. Neonatal Vaccination: Challenges and Intervention Strategies. Neonatology 109, 161-169, doi:10.1159/000442460 (2016).

23 Maisonneuve, C., Bertholet, S., Philpott, D. J. \& De Gregorio, E. Unleashing the potential of NOD- and Tolllike agonists as vaccine adjuvants. Proceedings of the National Academy of Sciences of the United States of America 111, 12294-12299, doi:10.1073/pnas.1400478111 (2014).

24 Auray, G. et al. Characterization and Transcriptomic Analysis of Porcine Blood Conventional and Plasmacytoid Dendritic Cells Reveals Striking Species-Specific Differences. Journal of immunology 197, 4791-4806, doi:10.4049/jimmunol.1600672 (2016).

25 Braun, R. O., Python, S. \& Summerfield, A. Porcine B Cell Subset Responses to Toll-like Receptor Ligands. Frontiers in immunology 8, 1044, doi:10.3389/fimmu.2017.01044 (2017).

26 Vreman, S. et al. Neonatal porcine blood derived dendritic cells subsets show activation after TLR2 or TLR9 stimulation. Developmental and comparative immunology, doi:10.1016/j.dci.2018.03.012 (2018).

27 Vreman, S. et al. Toll-like receptor agonists as adjuvants for inactivated porcine reproductive and respiratory syndrome virus (PRRSV) vaccine. Veterinary immunology and immunopathology 212, 27-37, doi:10.1016/j. vetimm.2019.04.008 (2019).

Matthijs, A. M. F.et al. Systems Immunology Characterization of Novel Vaccine Formulations for Mycoplasma hyopneumoniae Bacterins. Frontiers in immunology 10, 1087, doi:10.3389/fimmu.2019.01087 (2019). van Aalst, S. et al. Routing dependent immune responses after experimental R848-adjuvated vaccination. Vaccine 36, 1405-1413, doi:10.1016/j.vaccine.2018.01.077 (2018).

30 McKay, P. F. et al. TLR4 and TLR7/8 Adjuvant Combinations Generate Different Vaccine Antigen-Specific Immune Outcomes in Minipigs when Administered via the ID or IN Routes. PloS one 11, e0148984, doi:10.1371/journal.pone.0148984 (2016).

31 Vrdoljak, A. et al. Induction of broad immunity by thermostabilised vaccines incorporated in dissolvable microneedles using novel fabrication methods. Journal of controlled release : official journal of the Controlled Release Society 225, 192-204, doi:10.1016/j.jconrel.2016.01.019 (2016).

32 Ferrari, L. et al. Lymphocyte activation as cytokine gene expression and secretion is related to the porcine reproductive and respiratory syndrome virus (PRRSV) isolate after in vitro homologous and heterologous 
recall of peripheral blood mononuclear cells (PBMC) from pigs vaccinated and exposed to natural infection. Veterinary immunology and immunopathology 151, 193-206, doi:10.1016/j.vetimm.2012.11.006 (2013).

33 Wang, Y. et al. Skin Vaccination against Rotavirus Using Microneedles: Proof of Concept in Gnotobiotic Piglets. PloS one 11, e0166038, doi:10.1371/journal.pone.0166038 (2016).

34 Eble, P. L., Weerdmeester, K., van Hemert-Kluitenberg, F. \& Dekker, A. Intradermal vaccination of pigs against FMD with $1 / 10$ dose results in comparable vaccine efficacy as intramuscular vaccination with a full dose. Vaccine 27, 1272-1278, doi:10.1016/j.vaccine.2008.12.011 (2009).

35 Marshall, S., Sahm, L. J. \& Moore, A. C. The success of microneedle-mediated vaccine delivery into skin. Hum. Vaccin. Immunother., 0, doi:10.1080/21645515.2016.1171440 (2016).

36 Le Luduec, J. B. et al. Intradermal vaccination with un-adjuvanted sub-unit vaccines triggers skin innate immunity and confers protective respiratory immunity in domestic swine. Vaccine 34, 914-922, doi:10.1016/j.vaccine.2015.12.058 (2016).

37 Martelli, P.et al. Systemic and local immune response in pigs intradermally and intramuscularly injected with inactivated Mycoplasma hyopneumoniae vaccines. Veterinary microbiology 168, 357-364, doi:10.1016/j. vetmic.2013.11.025 (2014).

38 Ventura, R. et al. Technology transfer of an oil-in-water vaccine-adjuvant for strengthening pandemic influenza preparedness in Indonesia. Vaccine 31, 1641-1645, doi:10.1016/j.vaccine.2012.07.074 (2013).

39 Shah, R. R. et al. The development of self-emulsifying oil-in-water emulsion adjuvant and an evaluation of the impact of droplet size on performance. Journal of pharmaceutical sciences 104, 1352-1361, doi:10.1002/ jps.24337 (2015).

40 Mateu de Antonio, E. et al. Quantitative detection of porcine interferon-gamma in response to mitogen, superantigen and recall viral antigen. Veterinary immunology and immunopathology 61, 265-277, doi:10.1016/s0165-2427(97)00141-4 (1998).

41 Gerner, W. et al. Phenotypic and functional differentiation of porcine alphabeta T cells: current knowledge and available tools. Molecular immunology 66, 3-13, doi:10.1016/j.molimm.2014.10.025 (2015).

42 Sinkora, M., Stepanova, K. \& Sinkorova, J. Different anti-CD21 antibodies can be used to discriminate developmentally and functionally different subsets of B lymphocytes in circulation of pigs. Developmental and comparative immunology 39, 409-418, doi:10.1016/j.dci.2012.10.010 (2013).

43 Leroux-Roels, G. Unmet needs in modern vaccinology: adjuvants to improve the immune response. Vaccine 28 Suppl 3, C25-36, doi:10.1016/j.vaccine.2010.07.021 (2010).

44 O'Neill, K. C. et al. Studies on porcine circovirus type 2 vaccination of 5-day-old piglets. Clinical and vaccine immunology: CVI 18, 1865-1871, doi:10.1128/cvi.05318-11 (2011).

45 Auray, G. et al. Porcine neonatal blood dendritic cells, but not monocytes, are more responsive to TLRs stimulation than their adult counterparts. PloS one 8, e59629, doi:10.1371/journal.pone.0059629 (2013).

46 Medzhitov, R. Toll-like receptors and innate immunity. Nature Reviews Immunology 1, 135-145, doi:10.1038/35100529 (2001).

47 Rojas, J. M., Avia, M., Martin, V. \& Sevilla, N. IL-10: A Multifunctional Cytokine in Viral Infections. Journal of immunology research 2017, 6104054, doi:10.1155/2017/6104054 (2017).

48 Surendran, N., Simmons, A. \& Pichichero, M. E. TLR agonist combinations that stimulate Th type I polarizing responses from human neonates. Innate immunity $\mathbf{2 4 ,}$ 240-251, doi:10.1177/1753425918771178 (2018). 
49 Makinen, S. R., Zhu, Q., Davis, H. L. \& Weeratna, R. D. CpG-mediated augmentation of CD8+ T-cell responses in mice is attenuated by a water-in-oil emulsion (Montanide ISA-51) but enhanced by an oil-in-water emulsion (IDRI SE). International immunology 28, 453-461, doi:10.1093/intimm/dxw017 (2016).

50 Lee, H. J., Kim, K. C., Han, J. A., Choi, S. S. \& Jung, Y. J. The early induction of suppressor of cytokine signaling 1 and the downregulation of toll-like receptors 7 and 9 induce tolerance in costimulated macrophages. Molecules and cells 38, 26-32, doi:10.14348/molcells.2015.2136 (2015).

51 Holbrook, B. C. et al. A Novel R848-Conjugated Inactivated Influenza Virus Vaccine Is Efficacious and Safe in a Neonate Nonhuman Primate Model. Journal of immunology 197, 555-564, doi:10.4049/jimmunol.1600497 (2016).

$52 \mathrm{Ke}, \mathrm{H}$. \& Yoo, D. The viral innate immune antagonism and an alternative vaccine design for PRRS virus. Veterinary microbiology 209, 75-89, doi:10.1016/j.vetmic.2017.03.014 (2017).

53 Aucouturier, J., Dupuis, L. \& Ganne, V. Adjuvants designed for veterinary and human vaccines. Vaccine 19, 2666-2672 (2001).

54 Martinon, S. et al. Chemical and Immunological Characteristics of Aluminum-Based, Oil-Water Emulsion, and Bacterial-Origin Adjuvants. Journal of immunology research 2019, 3974127, doi:10.1155/2019/3974127 (2019).

55 Lee, J. A. et al. Protective humoral immune response induced by an inactivated porcine reproductive and respiratory syndrome virus expressing the hypo-glycosylated glycoprotein 5. Vaccine 32, 3617-3622, doi:10.1016/j.vaccine.2014.04.083 (2014).

56 Younis, S. Y. et al. Down selecting adjuvanted vaccine formulations: a comparative method for harmonized evaluation. BMC immunology 19, 6, doi:10.1186/s12865-018-0245-0 (2018).

57 Martelli, P. et al. Efficacy of a modified live porcine reproductive and respiratory syndrome virus (PRRSV) vaccine in pigs naturally exposed to a heterologous European (Italian cluster) field strain: Clinical protection and cell-mediated immunity. Vaccine 27, 3788-3799, doi:10.1016/j.vaccine.2009.03.028 (2009).

58 Meier, W. A. et al. Gradual development of the interferon-gamma response of swine to porcine reproductive and respiratory syndrome virus infection or vaccination. Virology 309, 18-31 (2003).

59 Weesendorp, E. et al. Comparative analysis of immune responses following experimental infection of pigs with European porcine reproductive and respiratory syndrome virus strains of differing virulence. Veterinary microbiology 163, 1-12, doi:10.1016/j.vetmic.2012.09.013 (2013).

60 Black, C. A. Delayed type hypersensitivity: current theories with an historic perspective. Dermatology online journal 5, 7 (1999).

61 Heinen, P. P., Rijsewijk, F. A., de Boer-Luijtze, E. A. \& Bianchi, A. T. Vaccination of pigs with a DNA construct expressing an influenza virus M2-nucleoprotein fusion protein exacerbates disease after challenge with influenza A virus. The Journal of general virology 83, 1851-1859, doi:10.1099/0022-1317-83-8-1851 (2002).

62 Dowling, D. J.et al. TLR7/8 adjuvant overcomes newborn hyporesponsiveness to pneumococcal conjugate vaccine at birth. JCl insight 2, e91020, doi:10.1172/jci.insight.91020 (2017).

63 Ganapathi, L. et al. The Imidazoquinoline Toll-Like Receptor-7/8 Agonist Hybrid-2 Potently Induces Cytokine Production by Human Newborn and Adult Leukocytes. PloS one 10, e0134640, doi:10.1371/ journal.pone.0134640 (2015). 
General discussion 

In this thesis I investigated the immune-stimulatory effects of Toll-like receptor (TLR) agonists on local and generalized immune responses in neonatal and adult pigs. The inactivated porcine reproductive and respiratory syndrome virus (PRRSV) antigen was combined with different TLR agonists (TLRa) to enhance the immunogenic potential of the PRRSV-vaccine, which was administered intramuscularly (i.m.) or by skin vaccination. The following research questions will be addressed in order to discuss the results of this thesis in a broader perspective:

1. Are the immune responses after TLR stimulation or PRRSV vaccination age-dependent?

2. Are TLR agonists able to enhance the specific immune response of the PRRSV vaccine?

3. Does skin administration of the PRRSV vaccine provide an enhanced immune response compared to conventional i.m. vaccination?

Finally, I will discuss the analysis of the vaccine-specific immune responses (section 4) and the societal relevance of this thesis (section 5).

\section{Age-dependent immune responses after TLR-stimulation and/or PRRSV vaccination}

The different immuno-stimulators or adjuvants used in our in vitro and vaccine studies induced different innate and adaptive immune responses in neonatal pigs compared to adult pigs (chapters 2-5). Table 1 provides an overview of the immune responses induced by the different immuno-stimulators used in both age groups:TLR1/2a, TLR7/8a, TLR9a, and the reference O/W adjuvant Montanide ${ }^{\mathrm{TM}}$ ISA28 (ISA28). The level of the neonatal immune responses was compared to the adult immune responses. The most relevant adjuvanted neonatal and adult immune responses will be discussed in the next paragraphs.

\section{Toll-like receptor agonists}

TLR1/2 and TLR7/8 stimulation induces a pro-inflammatory environment, especially in neonatal pigs. After TLR1/2 stimulation, neonatal mononuclear cells produced higher levels of the pro-inflammatory IL- 6 and expressed more TLR2 compared to the adult cells (chapter 2). After skin vaccination with TLR1/2a, and especially the TLR7/8a, a higher number of pro-inflammatory genes were upregulated compared to the non-vaccinated skin in neonatal pigs than in adult pigs (chapter 3). Interestingly, only the neonatal pigs showed an upregulation of TLR2 and TLR7 gene expression in the skin after vaccination with the TLR1/2a or TLR7/8a, which was also observed for TLR2 gene expression after TLR2 stimulation (chapter 2). This TLR upregulation after TLR-specific stimulation enables neonates to maintain their pro-inflammatory environment after TLR re-stimulation, e.g. in order to control an infection. Studies in mice and humans ${ }^{1,2}$ showed that high levels of pro-inflammatory cytokines (II-6, TNF and IL-8) were more easily generated in neonates, especially after TLR1/2 and TLR7/8 stimulation ${ }^{3,4}$, and these pro-inflammatory cytokines 
can activate the adaptive immune response. However, these high cytokine levels can also work as a double-edged sword in (pre-term) neonates by inducing exaggerated inflammation ${ }^{5}$ due to enhanced neutrophil recruitment under the influence of IL- $8{ }^{6}$. That could potentially hamper effective vaccine responses at an early age ${ }^{7}$ or even be a safety issue ${ }^{2,8}$.

Besides pro-inflammatory cytokines, TLR1/2 and TLR7/8 stimulation induced high levels of the immuno-suppressive cytokine IL-10 after stimulation of neonatal peripheral blood mononuclear cells (PBMC) (chapter 5), which was also reported in human neonates ${ }^{9-11}$. Monocytes, DCs and T-cells are important producers of IL-10. In a pro-inflammatory environment, IL-10 is used to balance inflammation by suppression of antigen presentation, DC differentiation and T-cell function ${ }^{12}$. Additionally, TLR2 stimulation reduced the immuno-suppressive function of regulatory T-cells $\left(T_{R E G}\right)$, and promoted the differentiation of $T_{\text {REG }}$ in a Th17-like phenotype ${ }^{13}$, which could enhance the inflammatory conditions ${ }^{14}$. These immuno-suppressive responses after TLR1/2 and TLR7/8 stimulation could potentially mitigate the efficacy of vaccine responses in both age groups.

Both adult and neonatal pigs generated minimal Th1-directed cytokines after TLR1/2 stimulation. Only the TLR1/2a skin vaccine did not induce any regulation of gene expression in the Th response cluster in the skin of both neonatal and adult pigs, while these genes of the Th response cluster were upregulated with TR7/8a and downregulated with TLR9a adjuvanted vaccines (chapter 3). Additionally, TLR1/2 stimulation did not enhance the gene expression of Th1 cytokines IL-12 and IFN-a in either of the age groups (chapter 2), which was observed after TLR9 stimulation. In contrast, human studies ${ }^{15-17}$ suggested that a neonatal Th1 inducing adjuvant should contain a TLR2 agonists. However, Kativhu et al. (2016) used a bacterial antigen (Mycobacterium tuberculosis), which is an intracellular pathogen that already elicits a IFN-Y-mediated Th1, and IL-17 mediated Th17 immune response by itself ${ }^{18}$. Therefore, I conclude that TLR $1 / 2$ stimulation could be used to induce Th1 directed responses in neonates depending on the antigens, but not in combination with iPRRSV. 
Table 1: Adjuvanted immune responses in neonatal and adult pigs.

\begin{tabular}{|c|c|c|c|c|c|}
\hline \multicolumn{6}{|c|}{ Adjuvant formulations } \\
\hline Immune response & age & $\begin{array}{c}\text { TLR1/2a } \\
\text { Ch2,3 and } 4\end{array}$ & $\begin{array}{l}\text { TLR7/8a } \\
\text { Ch3 and } 4\end{array}$ & $\begin{array}{c}\text { TLR9a } \\
\text { Ch2,3 and } 4\end{array}$ & $\begin{array}{c}\text { ISA28 } \\
\text { Ch4 and } 5\end{array}$ \\
\hline \multirow[t]{2}{*}{ APC activation blood ${ }^{19}$} & $\mathrm{~N}$ & $++/ \uparrow$ & $+++/ \uparrow$ & $++/=$ & nd \\
\hline & $A$ & + & ++ & ++ & nd \\
\hline \multirow[t]{2}{*}{ Pro-inflammatory } & $\mathrm{N}$ & $++/ \uparrow$ & $+++/ \uparrow$ & $+/=$ & nd \\
\hline & $A$ & + & ++ & + & + \\
\hline \multirow[t]{2}{*}{ In situ skin response } & $\mathrm{N}$ & $+/=$ & $+++/=$ & $+/=$ & nd \\
\hline & $A$ & + & +++ & + & nd \\
\hline \multirow[t]{2}{*}{ Th1 response } & $\mathrm{N}$ & $\pm /=$ & $++/=$ & $++/=$ & $\pm /=$ \\
\hline & $A$ & \pm & ++ & ++ & \pm \\
\hline \multirow[t]{2}{*}{ Th2 response } & $\mathrm{N}$ & $\pm /=$ & $\pm /=$ & $\pm /=$ & $\pm / \downarrow$ \\
\hline & A & \pm & \pm & \pm & ++ \\
\hline \multirow[t]{2}{*}{ Reduction PRRSV } & $\mathrm{N}$ & nd & nd & nd & $0 / \downarrow$ \\
\hline & $A$ & 0 & 0 & ++ & ++ \\
\hline
\end{tabular}

Overview of the potency of the different adjuvants to stimulate the vaccine related immune responses in neonatal $(\mathrm{N})$ and adult pigs $(\mathrm{A})$ : absent $(0)$, minimal (-/+ not significant), minimal $(+)$, moderate $(++)$, strong $(+++)$ or not determined $(\mathrm{nd})$ ) supplemented with available porcine neonatal literature. Additionally, the neonatal immune responses were compared to the responses of the adult pigs (equal to adult pig $(=)$, higher $(\uparrow)$, lower $(\downarrow)$. Abbreviations: see appendix.

\section{TLR7/8 and TLR9 stimulation favours a Th1-directed response.}

In contrast to TLR1/2a, TLR7/8a and TLR9a activate the type I IFN stimulating IRF7 pathway ${ }^{20}$, which could play an important role in a further increase of this differential Th1 response by induction of an IFN-a response. Type I IFNs are not only important for an anti-viral response, but stimulate DCs as well as $C D 4^{+} \mathrm{T}$ cells, $\mathrm{CD} 8^{+} \mathrm{T}$ cells, $\mathrm{B}$ cells, and NK cells ${ }^{21}$. Induction of type I IFN cytokine, including IFN-a, would therefore be a useful property of vaccine adjuvants. The production and induction of Th1-directed cytokines (IFN-a, IFN-Y, and IL-12) was most prominent after administration of TLR7/8a R848 and TLR9a CpG ODN in both adults in neonates (chapter 2, $\mathbf{3}$ and 5) with comparable levels in both age groups. TLR7/8a-stimulated neonatal cord blood and TLR7/8a-stimulated neonatal primates ${ }^{22-24}$ showed at least similar levels of Th1-directed cytokines and Th1 CD4+ cells compared to their adult counterparts. Interestingly, the i.m. TLR9a-vaccine in adult pigs was the only TLRa-containing vaccine that reduced PRRSV viremia after challenge without a specific immune response after vaccination (chapter 4, this vaccine was not evaluated in neonatal pigs). A non-specific cellular immune response, measured with the IFN-Y ELISpot, was also only detected following i.m. TLR9a vaccination. Therefore this Th1-directed immune response could have contributed to the reduction of PRRSV viremia. 
Differences in basal TLR expression by immune cells of neonates and adults can influence the functional responses after TLR stimulation. Age-dependent differences in basal TLR-expression could be a prerequisite to divergent immune responses in neonatal and adult pigs. Comparable basal mRNA expression was found for TLR2 and TLR9 in peripheral blood mononuclear cells (PBMCs) of neonatal and adult pigs (chapter 2), which is similar to the expression levels of all TLRs reported in human neonatal and adult monocytes ${ }^{2,25}$. On the other hand, Auray et al. ${ }^{19}$ showed that TLR7 was higher expressed in neonatal blood derived dendritic cells (BDCs), which could enhance the neonatal response after TLR7 stimulation as we reported in our studies (chapter 3). However, caution is needed to extrapolate these results to our results, because the TLR expression was measured in different subsets of APCs and more research is needed to evaluate the protein expression in different APC subsets.

As an overall conclusion, especially TLR7/8a are promising immuno-stimulators in neonatal and adult pigs, because they induce both pro-inflammatory cytokines and a Th1 response. However, we should be aware that in neonatal pigs, TLR7/8 can induce high levels of pro- and anti-inflammatory cytokines, which could hamper an effective vaccine response or even could have a negative effect on porcine health because of the induction of an inflammatory response that could lead to unwanted tissue damage. After TLR1/2 stimulation, neonatal pigs were more responsive compared to adult pigs, however in both age groups the TLR1/2a did not induce a substantial local and systemic immune response after vaccination. Therefore, I suggest that the limited Th1 potential in both age groups combined with pro-inflammatory response in neonates makes the application of TLR1/2 agonists as an adjuvant for adult and neonatal pigs questionable. TLR9a did show potential in adult pigs to induce systemic and protective immune response, which was supported by other porcine studies using $\mathrm{CpG}$ as adjuvant ${ }^{26,27}$, but further investigation is needed for it possible use neonatal pigs.

\section{Oil-in-water emulsion Montanide ${ }^{\mathrm{TM}}$ ISA28}

\section{PRRSV vaccination with oil-in-water $(\mathrm{O} / \mathrm{W})$ adjuvant is less effective in neonates}

than in adults. For the PRRSV vaccine study in adults (chapter 4) and neonates (chapters 5), we used the same prime-boost-challenge model with the same vaccine-antigen in PRRSV-naive pigs. Based on the new insights from systemic vaccine responses in the adult vaccine study, we decided to use different adjuvant formulations with TLRs in the neonatal vaccine study. The O/W emulsion ISA28 was used as reference adjuvant in both the adult and the neonatal PRRSV-vaccination experiment. After ISA28 vaccination, a lower number of neonates developed a detectable humoral immune response compared to the adult vaccination experiment. On the other hand, 4 out of 9 animals developed a detectable antibody level after a prime vaccination with ISA28 at 3-days of age. This could indicate that neonatal pigs showed more variation in their immune response due to individual variation in the kinetics of immune system development while the immune system of 
adult pigs showed a more uniform level of the vaccination response. Additionally, the reduction of PRRSV viremia in the adult vaccine study with the ISA28 vaccine was not observed in the neonatal vaccine study. These results confirm that the antibody response most likely played a role in the reduction of the viremia after ISA28 adjuvanted vaccination in adult pigs, because we did not measure any differences in cellular immune response between adult and neonatal pigs before viral challenge. This is remarkable, because we expected that the cellular-immune response would be impaired in neonates due to a less developed Th1 response.

Maternal antibodies could make the difference. For all the studies in this thesis, we used PRRSV-naive pigs to optimize standardisation and to study the systemic effect of the different adjuvants without interference of maternal antibodies. PRRSV is a worldwide endemic disease ${ }^{28-30}$ and therefore a large number of neonatal pig receive PRRSV-specific maternal antibodies by colostrum intake ${ }^{31}$. These maternal antibodies are generally considered to mitigate the vaccine responses in neonates ${ }^{32}$ by controlling plasma cell and memory B-cell differentiation ${ }^{33}$. However, several porcine studies showed that the presence of maternal antibodies did not interfere with the vaccine responses ${ }^{34-37}$ or even positively influence the vaccine responses ${ }^{38-40}$. It was shown that the level of maternal antibodies is a major concern during vaccination of newborn piglets ${ }^{41,42}$, more than the age and the associated immaturity of the immune responsiveness. Further studies on the impact of maternally derived antibodies remain necessary.

In summary (question 1), the ISA28 adjuvant was less immunogenic when applied in neonatal pigs compared to adult pigs, indicating that neonatal pigs react differently to similar vaccination. Therefore, neonates could benefit from an additional or different immuno-stimulator to enhance the immune response to a comparable level as in adult pigs. We have shown that TLR7/8a could be a promising immuno-stimulator in an adjuvant formulation for neonatal pigs based on the induction of the innate and Th1 directed response and the early immune responses after skin vaccination (chapter $\mathbf{3}$ and 5). These findings are corroborated by several neonatal adjuvant studies with TLR7/8 agonists as discussed previously. The overall less potent results after TLR9a administration, and the more variable and less Th1-directed results with the TLR1/2a, indicate that these TLRa are less potent adjuvants and that further research is needed before including them in porcine neonatal vaccines.

For future research, I suggest to study the different polarizing Th1/Th17 versus $T_{\text {REG }}$ cytokine responses, which are especially important in neonatal immune responses and are to date not extensively investigated in neonatal pigs. Additionally, more knowledge about B-cell responses and germinal centre responses in the draining lymph node and spleen after vaccination would be valuable. Several studies showed that the less developed germinal centres in neonates, together with the low expression of B-cell receptors and impaired class-switching ${ }^{43} 44$, resulted in limited B-cell responses ${ }^{33,45}$, leading to low levels of primary lgG after infections and vaccination in neonates. Finally, further neonatal 
studies where PRRSV/antigen-specific maternal antibodies are included, are needed to elucidate the role of maternally derived antibodies in the vaccine-induced immune responses, including the vaccine-dependent cellular immune response at an early age.

\section{Are TLR agonists able to enhance the immune response of the PRRSV vaccine?}

Based on the in vitro results and the local immune responses $24 \mathrm{~h}$ after skin vaccination, we anticipated that the different TLRa would contribute to an effective immune response when applied in an iPRRSV vaccine. However, the TLR agonist in our vaccination experiments did not contribute to the induction of a specific immune response after booster vaccination at a comparable level as reached by the application of an O/W adjuvant (chapters 4 and 5). The fact that the TLRa were less successful as adjuvant in the iPRRSV vaccine may depend on several factors: the immune cells (including macrophages and dendritic cells) that were activated, their APC activation and maturation capacity, the homing ability of activated APCs to the draining lymph nodes and the subsequent antigen presentation in the context of MHC class II molecules, and finally the ability to recognize and activate naive CD4+ T-cells contributing to the development of a specific immune response. In the following paragraphs I will hypothesize why the TLRa formulations were less effective as adjuvant in the iPRRSV-vaccine than anticipated.

Immune cell recruitment at injection site drives systemic immune responses. Adjuvant mechanisms are complex and still not completely elucidated ${ }^{46}$. The action of $\mathrm{O} / \mathrm{W}$ adjuvants is driven by chemokine-induced immune cell recruitment at the injection site ${ }^{47}$. These recruited immune cells are primarily neutrophilic granulocytes, macrophages and inflammatory/monocyte-derived $\mathrm{DCs}{ }^{48}$, which potentially stimulate the development of Th1- and Th2-directed immune responses ${ }^{49,50}$. TLRa are potent immuno-stimulators, which target APCs ${ }^{51,52}$, however, the number and diversity of the recruited immune cells at the injection site with a TLRa adjuvanted vaccine are less evident compared to an O/W adjuvant. The absence of a significant immune cell recruitment at the skin vaccination site forTLR1/2a and TLR9a could have contributed to the lower generalized immune responses of these TLRa-adjuvanted vaccines compared to the O/W adjuvanted vaccine (chapter 3). Only TLR7/8a induced a significant local immune response at the skin vaccination site, which was characterized by the presence of mononuclear cells and neutrophilic granulocytes. However, this response could be restricted to skin vaccination, because we did not evaluate the injection site after i.m. vaccination, and TLR7/8a are known for their local skin responses after topical application ${ }^{53,54}$.

TLRa combined with O/W emulsion induced a pro-inflammatory environment, hampering specific immune responses. This inhibitory role on inducing a specific immune response could be especially true for the TLR combination with squalene based O/W emulsion (SWE+TLRa) (chapter 5). In this study, we measured a significant rise in body temperature at $6 \mathrm{~h}$ after the booster vaccination in the SWE+TLRa vaccine group 
compared to vaccine groups with only TLR or SWE as adjuvant (results not published). We speculate that this systemic pro-inflammatory condition resulted in a reduced cellularimmune response in the SWE+TLRa group compared to the SWE group. Only limited information is available about such a pro-inflammatory state induced shortly after vaccination where the TLRa are combined with an O/W emulsion. However, it is widely known that adjuvants in vaccines can induce a pro-inflammatory condition associated with fever or the induction of a cytokine storm, as was reported for TLR3a poly (I:C) and TLR7/8a in an influenza vaccine ${ }^{55}$. In addition, several studies showed that reducing such a systemic pro-inflammatory condition is clinically safer and resulted in a higher vaccine efficacy ${ }^{56,57}$. As previously mentioned in this discussion, $T_{\text {REG }}$ could play an important role, because a pro-inflammatory condition will induce $T_{R E G}$ to create an anti-inflammatory response, which is immunosuppressive and potentially hampers specific immune responses.

Combining different TLR agonists: less can be more. Applying a mixture of different TLRs in the vaccine could stimulate different pathways of immune cell activation and even result in synergy ${ }^{58}$. However, stimulation with multiple TLRa may also result in inhibitory effects on innate immune responses. Dual activation of the same pathway could down-regulate the innate immune response, as we (chapter 5) and other studies 59,60 observed reduced IFN-a production after dual TLR7/8 and TLR9 stimulation (IRF7 pathway) in neonatal PBMCs. The downregulation of vaccine responses by co-stimulation with closely related TLRs may thus be a regulatory mechanism by which the host prevents overstimulation of innate immune responses. This indicates that every TLR-combination can induce unique cytokine responses and that in vitro research is warranted before applying a certain TLRa combination in in vivo formulations to get more insight into/on the potential of a specific TLR combination.

PRRSV causes a delayed immune response. Besides the adjuvant, the antigen is the most important immune determining component of the vaccine formulation ${ }^{61,62}$. Infection with PRRSV can lead to a viremia with a poor production of IFN type I cytokines, resulting in a delayed and weak adaptive immune response ${ }^{63}$. Before clearing the virus with an effective immune response, pigs can carry and spread the virus up to 180 days after infection ${ }^{64}$. This delayed immune response enables the PRRSV to spread efficiently on swine farms, and at the same time makes it a difficult vaccine-antigen. This is even more true when the PRRSV virus is inactivated and viral epitopes that are possibly important for a protective immune response, are not conserved ${ }^{65}$. We selected the iPRRSV antigen throughout this thesis, and we consistently used the same antigen and antigen-dose to optimize the experimental setting to permit analyses and comparison of the immunostimulating properties of the different TLRa. The application of a more immunogenic antigen that would induce a more potent immune responses could have been used in our study to generate more discriminating results for the skin/ i.m. vaccination route 
comparison, the comparison between the neonate and adult vaccine response, and the potential differences between the individual TLRa induced vaccine responses.

In summary (question 2), in the context of this thesis it remains unknown to what extend vaccination efficacy is dependent on the induction of a pro-inflammatory environment that might provoke a compensatory immune suppression or induction of a state of tolerance. Such an environment, induced by the applied TLRa, can stimulate the migration of antigen containing DC to the draining lymph node, and subsequently induce or inhibit activation of naive CD4 ${ }^{+}$T-cells depending on the specific TLRa.

For future research, there is a need to investigate the local immune response with the possible influx of leucocytes at the site of vaccination, and to compare responses to O/W emulsion, TLRa and O/W emulsions combined with TLRa. Additionally, the extent of the pro-inflammatory environment induced shortly after TLR stimulation and the subsequently induced anti-inflammatory response by $\mathrm{T}_{\mathrm{REG}}$ and anti-inflammatory cytokines should be studied to create a better understanding of all the factors involved in the induction of the vaccine specific immune responses. Finally, vaccines with a tracerlabelled antigen could be used to identify cell populations in the draining lymph node, including DC subsets, containing this antigen. The amount of antigen and number of activated DCs are important factors determining the vaccine- induced specific CD4 ${ }^{+}$T-cell response in the draining lymph node.

\section{Does skin vaccination provide an enhanced immune response compared to traditional i.m. vaccination?}

The TLR7/8a skin vaccination induced the most prominent early immune responses in both adult and neonatal pigs (chapter 3). For both age groups the high upregulation (> 10-fold induction) for the genes in the APC activation/maturation cluster (CCL20, CD86, CXCL10,MX1), are indicative of APC activation and migration. However, no activation of LCs and dDCs was observed after TLR7/8a skin vaccination (chapter $\mathbf{3}$ ) in adult and neonatal pigs. Additionally, after repeated vaccination, the TLR7/8a skin vaccine induced no systemic specific immune responses or reduced PRRSV-viremia in adult pigs (chapter 4). Because skin vaccination and i.m vaccination induced comparable immune responses for the TLR7/8a-vaccine, the TLR1/2a vaccine (chapter 4) and the squalene O/W emulsion (SWE) combined with the TLRa mixture (SWE+TLRa) (chapter 5), we concluded that skin vaccination did not overcome the weak immunogenic potential of the iPRRSV-antigen.

The TLR9a skin vaccine (chapter 4 ) was the only skin vaccination, which did not induce a systemic immune response similar to the i.m. vaccination. This suggests that skin vaccination was less effective than i.m. vaccination with a TLR9a. I hypothesize that the absence of TLR9 gene expression in the skin (chapter 3), as was also observed in a human skin study ${ }^{66}$, limited the local immune responses in the skin. A limited number of genes in the APC activation/migration and acute phase inflammatory response cluster were mainly downregulated with the TLR9a skin vaccine, which was in contrast to the induced 
upregulation of a large number of genes by TLR7/8a. On the other hand, intradermal injection with a lower dose of TLR9a CpG ${ }^{67}$ induced clear upregulation of genes at the injection site, which may promote recruitment of innate immune cells and Th1 type cytokine production. Although, this intradermal injection was most likely applied in the subcutis, which could explain the differences in results for TLR9a skin vaccination. Also, murine studies using CpG ODN in cationic liposomes ${ }^{68,69}$ showed enhanced immune responses with DMN-patch vaccination. Therefore, I suggest that caution is needed when using TLR9a as adjuvant in porcine DMN-patches, and that encapsulation in liposomes of the vaccine could be a better alternative for the induction of a protective immune response.

Despite the limited systemic immune responses with DMN-patch vaccination, studies and reviews showed that skin vaccination induced quantitative and qualitative different immune responses compared to i.m. vaccination, even when using the same antigen and adjuvants ${ }^{70-74}$. All these studies, conducted in different species, supported the potential of skin vaccination, based on the large number of APCs and the high density of draining lymph vessels. To date the number of studies investigating the different types of APCS in neonatal porcine skin is limited. Therefore, we evaluated and compared the number APCs populations in the neonatal and adult porcine skin to identify possible quantitative difference in APCs, which could influence the effect of skin vaccination in neonates compared to adults (chapter $\mathbf{3}$ ) as addressed below.

\section{Skin vaccination in neonatal pigs}

The skin APCs are a heterogenous population of cells with functionally specialized subsets ${ }^{75,76}$. In this thesis, Langerhans cell (LCs) in the epidermis, dermal dendritic cells $(\mathrm{dDC})$, and macrophages $(M \phi)$ in the dermis were investigated. Analysis of these subsets is complicated by the absence of a single unique marker to define these subsets ${ }^{77,78}$. With the cell-surface markers applied in this thesis we showed in situ that the non-vaccinated (NV) adult and neonatal skin contained a comparable number of DCs, however the lower basal gene expression of MHCII, CD86 and CD101 in the neonatal skin compared to the adult skin suggested a lower activation status of the skin DCs in neonates.

In contrast, the neonatal dermis contained more $M \phi$ and showed a higher basal gene expression for CD163 compared to the adults. Although dermal M $\phi$ could be characterized as APC, they are more specialized towards phagocytosis, while DCs are more specialized in antigen presentation and activation of T cells ${ }^{79}$. When $M \phi$ process the vaccine-antigen at an earlier time-point than $\mathrm{dDC}$, they could even diminish the level of antigen presentation. Additionally, enhanced cytokine production (e.g. TNF) could be induced by neonatal dermal $M \phi^{80}$. Human neonatal keratinocytes showed also an enhanced gene expression of TNF, IL8 and CXCL10 (Iram et al., 2012) after TLR stimulation, which is comparable to our gene induction in whole skin samples after TLR7/8a vaccination. Higher TNF induction is 
important for LC activation and migration ${ }^{81,82}$ and could therefore be advantageous in neonatal skin vaccination.

Upregulation of expression of CCR7 on the cell-surface of DCs is associated with the ability of DCs to migrate to the draining lymph nodes by functional attraction to CCL2 ${ }^{81,83}$. This is predominantly present in $\mathrm{LCs}$ and $\mathrm{dDCs}{ }^{78}$, suggesting that mainly $\mathrm{LCs}$ and $\mathrm{dDCs}$ are important for vaccine-antigen presentation. Interestingly, with the most potent skin vaccine (TLR7/8a), only in neonates the expression of CCR7 was upregulated (10 foldinduction) and the upregulation of CCL20 was also higher in neonates compared to adults. This could indicate that neonatal DCs are more activated after TLR7/8a activation, because there was no difference in basal skin expression for CCR7 and CCL20 in adult and neonatal pigs.

Age-dependent functional differences after skin vaccination could be explained by divergence in basal gene expression in neonatal and adult control LNs. Neonatal LNs express a higher level of M $\phi$-related genes (CD163, CD14) and a lower level of DC-related genes (CD1a) compared to the adult LN. Neonatal LN expression is also more related towards a pro-inflammatory state with activated endothelium by higher expression of e.g. DEFB1, NOS and IL18 compared to adult LNs. As a result, there is a higher basal IL10 expression in the neonates, probably to counteract the pro-inflammatory condition. Moreover, it is hypothesised that the homing of immune cells to the tissues is assured by the higher expression of CCL8, ICAM and VCAM in neonatal LNs. It remains to be determined how these age-dependent differences influence the formation of systemic vaccine-specific immune responses and further research is required to identify the functional age-dependent differences of the APC subsets. Overall, the neonatal skin is a promising vaccination route, however, age-dependent immune responses at the vaccination site and within the draining $L N$ could result in divergent efficacy of skin vaccination in both age-groups.

In summary (question 3), the potential of skin vaccination to induce or enhance systemic immune responses was not proven in this thesis for both age groups. This could be due to the vaccine formulation, such as the weak iPRRSV antigen (section 2) or lack of liposome encapsulation, but also the absence of APC migration could be a limiting factor. On the other hand, the induced local immune response in the neonatal skin and the 'adult-like' habituation of DCs in the skin are encouraging results for the use of the skin as a vaccination route in neonatal pigs.

Future skin vaccination research could investigate the potential of ISA28 as adjuvant in DMN-patches with or without TLR7/8a and compare local and systemic immune responses with the i.m. vaccination route. For the local immune responses, it remains to be determined which cells infiltrated the dermis after vaccination. The number of $\mathrm{M} \phi$ or $\mathrm{CD}_{163^{+}}$cells did not increase after skin vaccination (chapter 3 ), while there was a significant influx of mainly mononuclear cells with a macrophage like morphology in the dermis. Most likely, CD163 was not expressed by these recently extravasated macrophages ${ }^{84,85}$. 
Additional cell-surface markers, besides CD14 and CD16 ${ }^{77,86}$, could provide more information about the ontogeny of the extravasated leucocytes.

\section{Measuring vaccine-specific immune responses}

The study-design and methods selected are essential to generate valid and reproducible vaccine-specific immune responses to confirm the research hypothesis. It is a complex process to design the "ideal" experiment, because many confounding variables need to be considered for both in vitro and in vivo research. A commercial PRRSV vaccine for neonatal pigs was not included in the neonatal vaccine study (chapter 5), because to date a registered commercial inactivated PRRSV vaccine is, to our knowledge, not available for neonatal pigs due to the limited immunogenic potential of such a vaccine in this age group. At the moment only two modified-live (MLV) vaccines are registered for 1-day old pigs: MLV-PRRSV-1, 1-day-old, ${ }^{87}$ and Frostera PRRS, 1-day-old, ${ }^{88}$, which can only be used in controlled stable conditions as these vaccines can induce viral shedding and influence the results of the other groups. The challenge with PRRSV and the adult and neonate comparison of the ISA28 group were used to validate the results of the neonatal vaccine study.

The challenge is an essential part of a (PRRSV) vaccination study. Especially when the correlates of protection are not well defined as holds true for PRRSV vaccines ${ }^{89}$, challenge with the relevant infectious agent is an essential part of a vaccination experiment. This was supported by the observed reduction of viremia in the i.m. TLR9avaccinated animals compared to NV animals (chapter 4), while a specific cellular and/ or humoral immune response was not detected after booster vaccination for this i.m. TLR9a vaccine. It remains unclear which immune response contributed to the reduced PRRSV viremia after i.m. TLR9a vaccination. This indicates that fundamental research of the interaction of PRRSV and the vaccine-antigen (iPRRSV) with the immune system is essential to allow further development of effective vaccines (correlates of protection). Below I give some suggestions for future research to identify relevant (PRRSV) vaccinerelated immune responses based on the knowledge obtained within the frame of this thesis.

T-cell responses: Especially memory T-cells are of importance to evaluate vaccine responses, but these memory $\mathrm{T}$ - cells are a heterogenous population and the proposed subsets are based on individual phenotypes, which may be functionally different ${ }^{90,91}$. Current techniques used to evaluate memoryT-cell responses (ELISpot and flow cytometry) are one-dimensional and evaluation of additional parameters, like different memory T-cell subsets, cytokine responses to evaluate T-cell polarisation and transcriptomics would give additional information ${ }^{92}$. Additional surface markers could be used to identify specific subsets within the memory T-cell population to permit evaluation of their intracellular cytokine expression, as has been described by the research group of Saalmuller ${ }^{91}$. Cytolytic porcine memory T-cells $\left(C D 4{ }^{+} C D 8 a^{+} C D 8 \beta^{+}\right)$can effectively kill a virus-infected cell after 
reinfection, $T$ effector memory cells $\left(C D 4^{+} \mathrm{CD} 8 \mathrm{a}^{+} \mathrm{CD} 27\right)$ rapidly produce different cytokines, while T-central memory cells $\left(\mathrm{CD} 4{ }^{+} \mathrm{CD} 8 \mathrm{a}^{+} \mathrm{CD} 27^{+}\right)$easily proliferate after reinfection. Apart from phenotypic analysis of memory T-cell subsets, functional responsiveness after in vitro or ex vivo stimulation should be evaluated, as memory is defined functionally ${ }^{93}$. These distinct memory T-cell subsets are relevant for an effective vaccine response, but further research is needed to investigate their functional contribution in vaccine immunity and especially whether they are age-dependent or not.

Additionally, the induction of regulatory T-cells $\left(\mathbf{T}_{\mathrm{REG}}\right)\left(\mathrm{CD} 4^{+} \mathrm{CD} 25^{+} \mathrm{FoxP} 3^{+}\right)$responses are important to evaluate after (PRRSV) vaccination, because this immunosuppressive T-cell subset, combined with the IL-10 they produce, has been shown to hamper T-cell proliferation and subsequent induction of protection against PRRSV ${ }^{94-96}$. Moreover, high levels of $T_{R E G}$ were associated with the induction of immunological tolerance, which could be the result of the thymic infection associated with PRRSV ${ }^{97}$. In a PRRSV review ${ }^{98}$ it was hypothesized that thymocyte development was altered after PRRSV infection, causing an acute deficiency in T-cells. This T-cell deficiency leads to'holes' in the T-cell repertoire, which could result in poor recognition of PRRSV and other pathogens in neonates. This research group stressed the importance of the thymus in PRRSV pathogenesis and suggested to compare the $L N$ germinal centres responses quantitatively and qualitatively in adult and neonatal pigs after vaccination and/or PRRSV infection. To study this, we need to expand the porcine toolbox with additional cell-surface-markers to identify follicular Th cells $\left(T_{F H}\right)$ within lymph nodes. This will contribute to a better understanding of the germinal centre response in neonatal pigs, which is most likely impaired as described for human neonates 99.

Finally, developments in the fields of systems immunology/biology/vaccinology 100,101 are of importance to evaluate vaccine responses and correlates of protection after vaccination. This is an emerging inter-disciplinary field, where multiple data derived from different "omics" analyses, such as transcriptomics, genomics, proteomics and metabolomics, are integrated to investigate the complex mechanisms of the immune responses after vaccination. In chapter $\mathbf{3}$, we used a system vaccinology approach with high throughput PCR data to investigate 74 genes within the vaccinated skin and draining lymph node. The altered gene expression levels of the different immune response clusters after vaccination provided us with novel insights in the different immune responses in adults and neonatal pigs employing skin vaccination as delivery route. The group of Summerfield started with transcriptomics-based systems biology in adult pigs ${ }^{102}$, using peripheral blood before vaccination and at several time-points after vaccination, to analyse changes within blood transcription modules (BTM). This approach was based on human peripheral blood ${ }^{103}$, and adapted for use in adult and immunocompetent pigs. Future vaccine research could adopt this approach in combination with the generated 
pathology and immunology data to evaluate PRRSV-specific immune responses and study protective immune responses in different age groups.

\section{Societal relevance of this research Human research}

The DC subsets and proportion of monocytes within adult and neonatal PBMCs and their immune responses after TLR stimulation showed many parallels with human research (chapter 2). Moreover, the adult and neonatal porcine skin contained a comparable DC subset (chapter 3) as was observed for human adult and neonatal skin ${ }^{104}$. In contrast, the DCs in neonatal mice skin differ phenotypically and functionally from human neonatal DCs 105,106 and specific genes, such as pro-inflammatory IL-8 ${ }^{107}$ and anti-inflammatory IOD1, are only expressed in human and pig skin ${ }^{108,109}$, and not in mouse skin. These similarities in structure and immune responses make the porcine neonatal ${ }^{110,111}$ and adult skin ${ }^{112,113} \mathrm{a}$ preferred models for human skin research. Besides the skin studies, pigs are an important model to study human (infectious) diseases because of the structural and functional similarities in the respiratory and digestive tracts and the systemic immune system ${ }^{76,114}$. The relevance of neonatal pigs to study human neonates has been recognized in several models for e.g. Bordetella pertussis, rotavirus, and neonatal acute respiratory distress syndrome ${ }^{115-117}$. With the upcoming systems biology approach, which generates a large amount of immune data in different species, the relevance of pigs as a human model will only increase.

\section{Porcine vaccine development}

Porcine vaccination is an important strategy to reduce the disease risk and improve the animal welfare on swine farms ${ }^{118}$. Moreover, environmental conditions and feed, which influence the stress level and susceptibility to diseases ${ }^{119-121}$, can also influence the immune responses. However, the protective effect of a vaccine against specific diseases cannot be replaced by interventions, which mainly focus on environment and/or feed. Therefore, future vaccine experiments could benefit from inclusion of additional parameters related to e.g. cage enrichment and feed additives

With the knowledge I gained during this thesis, I expect that formulating an effective iPRRSV vaccine for (newborn) pigs will continue to be a challenge for basic and applied porcine vaccinology and/or immunology. The results of this thesis are relevant for future porcine and also human vaccine development. In line with the literature, we showed that porcine neonatal immune responses are different from adult pigs. However, specific immuno-stimulants (adjuvants) induced equal or even enhanced immune responses in neonatal pigs compared to adult pigs. Therefore, further research is needed for specific vaccine adjuvants in neonatal pigs of which TLR7/8a is an interesting adjuvant candidate for both adult and neonatal vaccines. Moreover, after skin vaccination, the neonatal 
skin showed, to a certain extent, similar early immune responses at the vaccination site compared to the adult skin and therefore, in newborn pigs needle-free skin vaccination could be a promising vaccine delivery route. I suggest that skin vaccination of newborn pigs could be a practical strategy to prevent early-life infections on swine farms, when immunogenic antigens in combination with an immune-stimulator are specifically selected for neonatal pigs.

\section{Conclusion}

In this thesis different subsets of DCs were identified in neonatal blood and skin (chapters 2 and 3) that showed a high degree of similarity to their adult counterparts. After TLR stimulation, we observed age-specific immune responses, where TLR1/2a and especially TLR7/8a induced enhanced immune responses in neonatal pigs compared to adult pigs. Despite the measured in vitro immune responses in chapter $\mathbf{2}$, and the identified early immune responses in skin and draining lymph node in chapter $\mathbf{3}$, none of the TLRa containing vaccines with iPRRSV were able to induce a clearly detectable specific immune response after booster vaccination independent of the administration route (chapters 4 and 5). In the experiments reported in this thesis, vaccines with O/W adjuvants induced a stronger protective immune response compared to vaccines with TLRa only. Moreover, we also showed that addition of a combined TLRa mixture and an O/W adjuvant did not provide an added protective effect. In order for pharmaceutical companies to design novel porcine vaccines applicable in different ages, thorough research into the underlying immunological mechanisms is still required. 


\section{References}

1 Zhao, J. et al. Hyper innate responses in neonates lead to increased morbidity and mortality after infection. Proceedings of the National Academy of Sciences of the United States of America 105, 7528-7533, doi:10.1073/ pnas.0800152105 (2008).

2 Glaser, K. \& Speer, C. P. Toll-like receptor signaling in neonatal sepsis and inflammation: a matter of orchestration and conditioning. Expert Rev. Clin. Immunol. 9, 1239-1252, doi:10.1586/174466 6x.2013.857275 (2013).

3 Caron, J. E., La Pine, T. R., Augustine, N. H., Martins, T. B. \& Hill, H. R. Multiplex analysis of toll-like receptorstimulated neonatal cytokine response. Neonatology 97, 266-273, doi:10.1159/000255165 (2010).

4 Dreschers, S. et al. Reduced PICD in Monocytes Mounts Altered Neonate Immune Response to Candida albicans. PloS one 11, e0166648, doi:10.1371/journal.pone.0166648 (2016).

5 Wennekamp, J. \& Henneke, P. Induction and termination of inflammatory signaling in group B streptococcal sepsis. Immunol. Rev. 225, 114-127, doi:10.1111/j.1600-065X.2008.00673.x (2008).

6 Thornton, N. L., Cody, M. J. \& Yost, C. C. Toll-like receptor 1/2 stimulation induces elevated interleukin-8 secretion in polymorphonuclear leukocytes isolated from preterm and term newborn infants. Neonatology 101, 140-146, doi:10.1159/000330567 (2012).

7 Yang, J.et al. IL-6 Impairs Vaccine Responses in Neonatal Mice. Frontiers in immunology 9, 3049, doi:10.3389/ fimmu.2018.03049 (2018).

8 Demirjian, A. \& Levy, O. Safety and efficacy of neonatal vaccination. European journal of immunology 39, 36-46, doi:10.1002/eji.200838620 (2009).

9 Levy, O., Goriely, S. \& Kollmann, T. R. Immune response to vaccine adjuvants during the first year of life. Vaccine 31, 2500-2505, doi:10.1016/j.vaccine.2012.10.016 (2013).

10 Corbett, N. P. et al. Ontogeny of Toll-like receptor mediated cytokine responses of human blood mononuclear cells. PloS one 5, e15041, doi:10.1371/journal.pone.0015041 (2010).

11 Andrade, E. B. et al. TLR2-induced IL-10 production impairs neutrophil recruitment to infected tissues during neonatal bacterial sepsis. Journal of immunology 191, 4759-4768, doi:10.4049/jimmunol.1301752 (2013).

12 Rojas, J. M., Avia, M., Martin, V. \& Sevilla, N. IL-10: A Multifunctional Cytokine in Viral Infections. Journal of immunology research 2017, 6104054, doi:10.1155/2017/6104054 (2017).

13 Nyirenda, M. H. et al. TLR2 stimulation drives human naive and effector regulatory T cells into a Th17like phenotype with reduced suppressive function. Journal of immunology 187, 2278-2290, doi:10.4049/ jimmunol.1003715 (2011).

14 Kiros, T. G., van Kessel, J., Babiuk, L. A. \& Gerdts, V. Induction, regulation and physiological role of IL-17 secreting helper T-cells isolated from PBMC, thymus, and lung lymphocytes of young pigs. Veterinary immunology and immunopathology 144, 448-454, doi:10.1016/j.vetimm.2011.08.021 (2011).

15 Sinnott, B. D., Park, B., Boer, M. C., Lewinsohn, D. A. \& Lancioni, C. L. Direct TLR-2 Costimulation Unmasks the Proinflammatory Potential of Neonatal CD4+ T Cells. Journal of immunology 197, 68-77, doi:10.4049/ jimmunol.1501297 (2016).

16 Whittaker, E., Goldblatt, D., McIntyre, P. \& Levy, O. Neonatal Immunization: Rationale, Current State, and Future Prospects. Frontiers in immunology 9, 532, doi:10.3389/fimmu.2018.00532 (2018). 
17 Kativhu, C. L. \& Libraty, D. H. A Model to Explain How the Bacille Calmette Guerin (BCG) Vaccine Drives Interleukin-12 Production in Neonates. PloS one 11, e0162148, doi:10.1371/journal.pone.0162148 (2016).

18 Lyadova, I. V. \& Panteleev, A. V. Th1 and Th17 Cells in Tuberculosis: Protection, Pathology, and Biomarkers. Mediators Inflamm. 2015, 854507, doi:10.1155/2015/854507 (2015).

19 Auray, G. et al. Porcine neonatal blood dendritic cells, but not monocytes, are more responsive to TLRs stimulation than their adult counterparts. PloS one 8, e59629, doi:10.1371/journal.pone.0059629 (2013).

20 Uenishi, H. \& Shinkai, H. Porcine Toll-like receptors: the front line of pathogen monitoring and possible implications for disease resistance. Developmental and comparative immunology 33, 353-361, doi:10.1016/j. dci.2008.06.001 (2009).

$21 \mathrm{Ke}, \mathrm{H}$. \& Yoo, D. The viral innate immune antagonism and an alternative vaccine design for PRRS virus. Veterinary microbiology 209, 75-89, doi:10.1016/j.vetmic.2017.03.014 (2017).

22 Ganapathi, L. et al. The Imidazoquinoline Toll-Like Receptor-7/8 Agonist Hybrid-2 Potently Induces Cytokine Production by Human Newborn and Adult Leukocytes. PloS one 10, e0134640, doi:10.1371/ journal.pone.0134640 (2015).

23 Dowling, D. J.et al. TLR7/8 adjuvant overcomes newborn hyporesponsiveness to pneumococcal conjugate vaccine at birth. JCl insight 2, e91020, doi:10.1172/jci.insight.91020 (2017).

24 van Haren, S. D. et al. Age-Specific Adjuvant Synergy: Dual TLR7/8 and Mincle Activation of Human Newborn Dendritic Cells Enables Th1 Polarization. Journal of immunology 197, 4413-4424, doi:10.4049/ jimmunol.1600282 (2016).

25 Levy, O. et al. Selective impairment of TLR-mediated innate immunity in human newborns: neonatal blood plasma reduces monocyte TNF-alpha induction by bacterial lipopeptides, lipopolysaccharide, and imiquimod, but preserves the response to R-848. Journal of immunology 173, 4627-4634, doi:10.4049/ jimmunol.173.7.4627 (2004).

26 Van der Stede, Y., Verdonck, F., Vancaeneghem, S., Cox, E. \& Goddeeris, B. M. CpG-oligodinucleotides as an effective adjuvant in pigs for intramuscular immunizations. Veterinary immunology and immunopathology 86, 31-41 (2002).

27 Quan, Z. et al. Plasmid containing CpG oligodeoxynucleotides can augment the immune responses of pigs immunized with porcine reproductive and respiratory syndrome killed virus vaccine. Veterinary immunology and immunopathology 136, 257-264, doi:10.1016/j.vetimm.2010.03.015 (2010).

28 Frossard, J. P. \& Williamson, S. PRRS diagnoses in Great Britain 2016/17. The Veterinary record 182, 133-135, doi:10.1136/vr.k539 (2018).

29 Nodelijk, G., Nielen, M., De Jong, M. C. \& Verheijden, J. H. A review of porcine reproductive and respiratory syndrome virus in Dutch breeding herds: population dynamics and clinical relevance. Preventive veterinary medicine 60, 37-52, doi:10.1016/s0167-5877(03)00081-3 (2003).

30 Lopes Antunes, A. C. \& Jensen, D. Estimating time evolving cross-dependence of porcine reproduction and respiratory syndrome sero-prevalence in Danish swine herds. Preventive veterinary medicine 167, 85-89, doi:10.1016/j.prevetmed.2019.03.024 (2019).

31 Poonsuk, K. \& Zimmerman, J. Historical and contemporary aspects of maternal immunity in swine. Animal health research reviews, 1-15, doi:10.1017/s1466252317000123 (2017). 
32 Siegrist, C. A. Mechanisms by which maternal antibodies influence infant vaccine responses: review of hypotheses and definition of main determinants. Vaccine 21, 3406-3412 (2003).

33 Vono, M. et al. Maternal Antibodies Inhibit Neonatal and Infant Responses to Vaccination by Shaping the Early-Life B Cell Repertoire within Germinal Centers. Cell reports 28, 1773-1784 e1775, doi:10.1016/j. celrep.2019.07.047 (2019).

34 Wilson, S. et al. Vaccination of piglets up to 1 week of age with a single-dose Mycoplasma hyopneumoniae vaccine induces protective immunity within 2 weeks against virulent challenge in the presence of maternally derived antibodies. Clinical and vaccine immunology : CVI 20, 720-724, doi:10.1128/cvi.0007813 (2013).

35 Genzow, M., Goodell, C., Kaiser, T. J., Johnson, W. \& Eichmeyer, M. Live attenuated influenza virus vaccine reduces virus shedding of newborn piglets in the presence of maternal antibody. Influenza and other respiratory viruses 12, 353-359, doi:10.1111/irv.12531 (2018).

36 Kraft, C. et al. Evaluation of PRRSv specific, maternally derived and induced immune response in Ingelvac PRRSFLEX EU vaccinated piglets in the presence of maternally transferred immunity. PloS one 14, e0223060, doi:10.1371/journal.pone.0223060 (2019).

37 Balasch, M., Fort, M., Taylor, L. P. \& Calvert, J. G. Vaccination of 1-day-old pigs with a porcine reproductive and respiratory syndrome virus (PRRSV) modified live attenuated virus vaccine is able to overcome maternal immunity. Porcine health management 4, 25, doi:10.1186/s40813-018-0101-x (2018).

38 Guzman-Bautista, E. R. et al. Effect of age and maternal antibodies on the systemic and mucosal immune response after neonatal immunization in a porcine model. Immunology 141, 609-616, doi:10.1111/ imm.12222 (2014).

39 Bandrick, M., Theis, K. \& Molitor, T. W. Maternal immunity enhances Mycoplasma hyopneumoniae vaccination induced cell-mediated immune responses in piglets. BMC veterinary research 10, 124, doi:10.1186/1746-6148-10-124 (2014).

40 Dekker, A., Chenard, G., Stockhofe, N. \& Eble, P. L. Proper Timing of Foot-and-Mouth Disease Vaccination of Piglets with Maternally Derived Antibodies Will Maximize Expected Protection Levels. Frontiers in veterinary science 3, 52, doi:10.3389/fvets.2016.00052 (2016).

41 Hodgins, D. C., Shewen, P. E. \& Dewey, C. E. Influence of age and maternal antibodies on antibody responses of neonatal piglets vaccinated against Mycoplasma hyopneumoniae. Journal of Swine Health and Production 12, 10-16 (2004).

42 Hodgins, D. C. \& Shewen, P. E. Vaccination of neonates: problem and issues. Vaccine 30, 1541-1559, doi:10.1016/j.vaccine.2011.12.047 (2012).

43 Basha, S., Surendran, N. \& Pichichero, M. Immune responses in neonates. Expert Rev. Clin. Immunol. 10, 1171-1184, doi:10.1586/1744666x.2014.942288 (2014).

44 Glaesener, S. et al. Decreased production of class-switched antibodies in neonatal B cells is associated with increased expression of miR-181b. PloS one 13, e0192230, doi:10.1371/journal.pone.0192230 (2018).

45 Siegrist, C. A. \& Aspinall, R. B-cell responses to vaccination at the extremes of age. Nature reviews. Immunology 9, 185-194, doi:10.1038/nri2508 (2009).

46 Savelkoul, H. F., Ferro, V. A., Strioga, M. M. \& Schijns, V. E. Choice and Design of Adjuvants for Parenteral and Mucosal Vaccines. Vaccines 3, 148-171, doi:10.3390/vaccines3010148 (2015). 
47 Zhang, J. et al. Development of a novel oil-in-water emulsion and evaluation of its potential adjuvant function in a swine influenza vaccine in mice. BMC veterinary research 14, 415, doi:10.1186/s12917-0181719-2 (2018).

48 Shi, S. et al. Vaccine adjuvants: Understanding the structure and mechanism of adjuvanticity. Vaccine 37, 3167-3178, doi:10.1016/j.vaccine.2019.04.055 (2019).

49 McKee, A. S. \& Marrack, P. Old and new adjuvants. Current opinion in immunology 47, 44-51, doi:10.1016/j. coi.2017.06.005 (2017).

50 Fox, C. B. \& Haensler, J. An update on safety and immunogenicity of vaccines containing emulsion-based adjuvants. Expert review of vaccines 12, 747-758, doi:10.1586/14760584.2013.811188 (2013).

51 Steinhagen, F., Kinjo, T., Bode, C. \& Klinman, D. M. TLR-based immune adjuvants. Vaccine 29, 3341-3355, doi:10.1016/j.vaccine.2010.08.002 (2011).

52 Lahiri, A., Das, P. \& Chakravortty, D. Engagement of TLR signaling as adjuvant: towards smarter vaccine and beyond. Vaccine 26, 6777-6783, doi:10.1016/j.vaccine.2008.09.045 (2008).

53 Meyer, T., Surber, C., French, L. E. \& Stockfleth, E. Resiquimod, a topical drug for viral skin lesions and skin cancer. Expert opinion on investigational drugs 22, 149-159, doi:10.1517/13543784.2013.749236 (2013).

54 Zhang, W. W. \& Matlashewski, G. Immunization with a Toll-like receptor 7 and/or 8 agonist vaccine adjuvant increases protective immunity against Leishmania major in BALB/c mice. Infect. Immun. 76, 3777-3783, doi:10.1128/iai.01527-07 (2008).

55 Sasaki, E., Momose, H., Hiradate, Y., Mizukami, T. \& Hamaguchi, I. Establishment of a novel safety assessment method for vaccine adjuvant development. Vaccine 36, 7112-7118, doi:10.1016/j.vaccine.2018.10.009 (2018).

56 Patel, S. et al. Comparative Safety and Efficacy Profile of a Novel Oil in Water Vaccine Adjuvant Comprising Vitamins A and E and a Catechin in Protective Anti-Influenza Immunity. Nutrients 9, doi:10.3390/nu9050516 (2017).

57 Nordly, P. et al. Immunity by formulation design: induction of high CD8+ T-cell responses by poly(l:C) incorporated into the CAF01 adjuvant via a double emulsion method. Journal of controlled release : official journal of the Controlled Release Society 150, 307-317, doi:10.1016/j.jconrel.2010.11.021 (2011).

58 Tan, R. S., Ho, B., Leung, B. P. \& Ding, J. L. TLR cross-talk confers specificity to innate immunity. International reviews of immunology 33, 443-453, doi:10.3109/08830185.2014.921164 (2014).

59 Surendran, N., Simmons, A. \& Pichichero, M. E. TLR agonist combinations that stimulate Th type I polarizing responses from human neonates. Innate immunity 24, 240-251, doi:10.1177/1753425918771178 (2018).

60 Booth, J. S., Buza, J. J., Potter, A., Babiuk, L. A. \& Mutwiri, G. K. Co-stimulation with TLR7/8 and TLR9 agonists induce down-regulation of innate immune responses in sheep blood mononuclear and B cells. Dev. Comp. Immunol. 34, 572-578, doi:10.1016/j.dci.2009.12.018 (2010).

61 Slifka, M. K. \& Amanna, I. How advances in immunology provide insight into improving vaccine efficacy. Vaccine 32, 2948-2957, doi:10.1016/j.vaccine.2014.03.078 (2014).

62 Zinkernagel, R. M. On natural and artificial vaccinations. Annual review of immunology 21, 515-546, doi:10.1146/annurev.immunol.21.120601.141045 (2003). 
63 Loving, C. L., Osorio, F. A., Murtaugh, M. P. \& Zuckermann, F. A. Innate and adaptive immunity against Porcine Reproductive and Respiratory Syndrome Virus. Veterinary immunology and immunopathology 167, 1-14, doi:10.1016/j.vetimm.2015.07.003 (2015).

64 Allende, R. et al. Porcine reproductive and respiratory syndrome virus: description of persistence in individual pigs upon experimental infection. Journal of virology 74, 10834-10837, doi:10.1128/ jvi.74.22.10834-10837.2000 (2000).

65 Moreno, S. et al. Analysis of chemokine receptor CCR7 expression on porcine blood T lymphocytes using a CCL19-Fc fusion protein. Developmental and comparative immunology 39, 207-213, doi:10.1016/j. dci.2012.11.010 (2013).

66 Iram, N. et al. Age-related changes in expression and function of Toll-like receptors in human skin. Development 139, 4210-4219, doi:10.1242/dev.083477 (2012).

67 Magiri,R.B.etal.Responseofimmuneresponsegenestoadjuvantspoly[di(sodiumcarboxylatoethylphenoxy) phosphazene] (PCEP), CpG oligodeoxynucleotide and emulsigen at intradermal injection site in pigs. Veterinary immunology and immunopathology 175, 57-63, doi:10.1016/j.vetimm.2016.05.003 (2016).

68 Guo, L. et al. Enhanced transcutaneous immunization via dissolving microneedle array loaded with liposome encapsulated antigen and adjuvant. Int. J. Pharm. 447, 22-30, doi:10.1016/j.ijpharm.2013.02.006 (2013).

69 Qiu, Y. et al. DNA-based vaccination against hepatitis B virus using dissolving microneedle arrays adjuvanted by cationic liposomes and CpG ODN. Drug Deliv 23, 2391-2398, doi:10.3109/10717544.2014.9 92497 (2016).

70 Filippelli, M. et al. Hepatitis B vaccine by intradermal route in non responder patients: an update. World J. Gastroenterol. 20, 10383-10394, doi:10.3748/wjg.v20.i30.10383 (2014).

71 Tassis, P. D. et al. Clinical evaluation of intradermal vaccination against porcine enzootic pneumonia (Mycoplasma hyopneumoniae). The Veterinary record 170, 261, doi:10.1136/vr.100239 (2012).

72 Niu, L., Chu, L. Y., Burton, S. A., Hansen, K. J. \& Panyam, J. Intradermal delivery of vaccine nanoparticles using hollow microneedle array generates enhanced and balanced immune response. Journal of controlled release : official journal of the Controlled Release Society 294, 268-278, doi:10.1016/j.jconrel.2018.12.026 (2019).

73 Martelli, P. et al. Efficacy of a modified live porcine reproductive and respiratory syndrome virus (PRRSV) vaccine in pigs naturally exposed to a heterologous European (Italian cluster) field strain: Clinical protection and cell-mediated immunity. Vaccine 27, 3788-3799, doi:10.1016/j.vaccine.2009.03.028 (2009).

74 Leone, M., Monkare, J., Bouwstra, J. A. \& Kersten, G. Dissolving Microneedle Patches for Dermal Vaccination. Pharm. Res. 34, 2223-2240, doi:10.1007/s11095-017-2223-2 (2017).

75 Kashem, S. W., Haniffa, M. \& Kaplan, D. H. Antigen-Presenting Cells in the Skin. Annual review of immunology 35, 469-499, doi:10.1146/annurev-immunol-051116-052215 (2017).

76 Summerfield, A., Meurens, F. \& Ricklin, M. E. The immunology of the porcine skin and its value as a model for human skin. Molecular immunology 66, 14-21, doi:10.1016/j.molimm.2014.10.023 (2015).

77 Marquet, F. et al. Characterization of dendritic cells subpopulations in skin and afferent lymph in the swine model. PloS one 6, e16320, doi:10.1371/journal.pone.0016320 (2011). 
78 Marquet, F. et al. Pig skin includes dendritic cell subsets transcriptomically related to human CD1a and CD14 dendritic cells presenting different migrating behaviors and T cell activation capacities. Journal of immunology 193, 5883-5893, doi:10.4049/jimmunol.1303150 (2014).

79 de Jong, J. M. et al. Dendritic cells, but not macrophages or B cells, activate major histocompatibility complex class II-restricted CD4+ T cells upon immune-complex uptake in vivo. Immunology 119, 499-506, doi:10.1111/j.1365-2567.2006.02464.x (2006).

80 Parameswaran, N. \& Patial, S. Tumor necrosis factor-alpha signaling in macrophages. Crit. Rev. Eukaryot. Gene Expr. 20, 87-103, doi:10.1615/critreveukargeneexpr.v20.i2.10 (2010).

81 Forster, R., Braun, A. \& Worbs, T. Lymph node homing of T cells and dendritic cells via afferent lymphatics. Trends in immunology 33, 271-280, doi:10.1016/j.it.2012.02.007 (2012).

82 Berthier-Vergnes, O. et al. TNF-alpha enhances phenotypic and functional maturation of human epidermal Langerhans cells and induces IL-12 p40 and IP-10/CXCL-10 production. FEBS Lett. 579, 3660-3668, doi:10.1016/j.febslet.2005.04.087 (2005).

83 Banchereau, J. et al. Immunobiology of Dendritic Cells. Annu. Rev. Immunol. 18, 767-811, doi:10.1146/ annurev.immunol.18.1.767 (2000).

84 Fabriek, B. O., Dijkstra, C. D. \& van den Berg, T. K. The macrophage scavenger receptor CD163. Immunobiology 210, 153-160, doi:10.1016/j.imbio.2005.05.010 (2005).

85 Liu, Q. et al. Differential expression and predictive value of monocyte scavenger receptor CD163 in populations with different tuberculosis infection statuses. BMC Infect. Dis. 19, 1006, doi:10.1186/s12879019-4525-y (2019).

86 Collin, M. \& Bigley, V. Monocyte, Macrophage, and Dendritic Cell Development: the Human Perspective. Microbiol Spectr 4, doi:10.1128/microbiolspec.MCHD-0015-2015 (2016).

87 Balasch, M. et al. Immune response development after vaccination of 1-day-old naive pigs with a Porcine Reproductive and Respiratory Syndrome 1-based modified live virus vaccine. Porcine health management 5, 2, doi:10.1186/s40813-018-0112-7 (2019).

88 Jeong, J. et al. Vaccination with a porcine reproductive and respiratory syndrome virus vaccine at 1-dayold improved growth performance of piglets under field conditions. Veterinary microbiology 214, 113-124, doi:10.1016/j.vetmic.2017.12.023 (2018).

89 Vu, H. L. X., Pattnaik, A. K. \& Osorio, F. A. Strategies to broaden the cross-protective efficacy of vaccines against porcine reproductive and respiratory syndrome virus. Veterinary microbiology 206, 29-34, doi:10.1016/j.vetmic.2016.09.014 (2017).

90 Jameson, S. C. \& Masopust, D. Understanding Subset Diversity in T Cell Memory. Immunity 48, 214-226, doi:10.1016/j.immuni.2018.02.010 (2018).

91 Gerner, W. et al. Phenotypic and functional differentiation of porcine alphabeta T cells: current knowledge and available tools. Molecular immunology 66, 3-13, doi:10.1016/j.molimm.2014.10.025 (2015).

92 Flaxman, A. \& Ewer, K. J. Methods for Measuring T-Cell Memory to Vaccination: From Mouse to Man. Vaccines 6, doi:10.3390/vaccines6030043 (2018).

93 Albert-Vega, C. et al. Immune Functional Assays, From Custom to Standardized Tests for Precision Medicine. Frontiers in immunology 9, 2367, doi:10.3389/fimmu.2018.02367 (2018). 
94 Wongyanin, P. et al. Role of porcine reproductive and respiratory syndrome virus nucleocapsid protein in induction of interleukin-10 and regulatory T-lymphocytes (Treg). The Journal of general virology 93, 12361246, doi:10.1099/vir.0.040287-0 (2012).

95 Montaner-Tarbes, S., Del Portillo, H. A., Montoya, M. \& Fraile, L. Key Gaps in the Knowledge of the Porcine Respiratory Reproductive Syndrome Virus (PRRSV). Frontiers in veterinary science 6, 38, doi:10.3389/ fvets.2019.00038 (2019).

96 Lunney, J. K. et al. Porcine Reproductive and Respiratory Syndrome Virus (PRRSV): Pathogenesis and Interaction with the Immune System. Annual review of animal biosciences 4, 129-154, doi:10.1146/annurevanimal-022114-111025 (2016).

97 Butler, J. E. et al. Porcine reproductive and respiratory syndrome (PRRS): an immune dysregulatory pandemic. Immunol. Res. 59, 81-108, doi:10.1007/s12026-014-8549-5 (2014).

98 Butler, J. E. et al. Perturbation of Thymocyte Development Underlies the PRRS Pandemic: A Testable Hypothesis. Frontiers in immunology 10, 1077, doi:10.3389/fimmu.2019.01077 (2019).

99 Vono, M. et al. Overcoming the Neonatal Limitations of Inducing Germinal Centers through LiposomeBased Adjuvants Including C-Type Lectin Agonists Trehalose Dibehenate or Curdlan. Frontiers in immunology 9, 381, doi:10.3389/fimmu.2018.00381 (2018).

100 Olafsdottir, T., Lindqvist, M. \& Harandi, A. M. Molecular signatures of vaccine adjuvants. Vaccine 33, 53025307, doi:10.1016/j.vaccine.2015.04.099 (2015).

101 Hagan, T., Nakaya, H. I., Subramaniam, S. \& Pulendran, B. Systems vaccinology: Enabling rational vaccine design with systems biological approaches. Vaccine 33, 5294-5301, doi:10.1016/j.vaccine.2015.03.072 (2015).

102 Matthijs, A. M. F.et al. Systems Immunology Characterization of Novel Vaccine Formulations for Mycoplasma hyopneumoniae Bacterins. Frontiers in immunology 10, 1087, doi:10.3389/fimmu.2019.01087 (2019).

$103 \mathrm{Li}$, S. et al. Molecular signatures of antibody responses derived from a systems biology study of five human vaccines. Nature immunology 15, 195-204, doi:10.1038/ni.2789 (2014).

104 Drijkoningen, M., De Wolf-Peeters, C., Van der Steen, K., Moerman, P. \& Desmet, V. Epidermal Langerhans' cells and dermal dendritic cells in human fetal and neonatal skin: an immunohistochemical study. Pediatric dermatology 4, 11-17, doi:10.1111/j.1525-1470.1987.tb00745.x (1987).

105 Elbe-Burger, A. \& Schuster, C. Development of the prenatal cutaneous antigen-presenting cell network. Immunology and cell biology 88, 393-399, doi:10.1038/icb.2010.13 (2010).

106 Dewar, A. L., Doherty, K. V., Woods, G. M., Lyons, A. B. \& Muller, H. K. Acquisition of immune function during the development of the Langerhans cell network in neonatal mice. Immunology 103, 61-69, doi:10.1046/ j.1365-2567.2001.01221.x (2001).

107 Asfaha, S. et al. Mice that express human interleukin-8 have increased mobilization of immature myeloid cells, which exacerbates inflammation and accelerates colon carcinogenesis. Gastroenterology 144, 155166, doi:10.1053/j.gastro.2012.09.057 (2013).

108 Dawson, H., A Comparative Assessment of the Pig, Mouse and Human Genomes, pp. 323-342 (2011).

109 Dawson, H. D., Smith, A. D., Chen, C. \& Urban, J. F., Jr. An in-depth comparison of the porcine, murine and human inflammasomes; lessons from the porcine genome and transcriptome. Veterinary microbiology 202, 2-15, doi:10.1016/j.vetmic.2016.05.013 (2017). 
110 Gauthier, B. E., Penard, L., Bordier, N. F., Briffaux, J. J. \& Ruty, B. M. Specificities of the Skin Morphology in Juvenile Minipigs. Toxicologic pathology 46, 821-834, doi:10.1177/0192623318804520 (2018).

111 Simon, G. A. \& Maibach, H. I. The pig as an experimental animal model of percutaneous permeation in man: qualitative and quantitative observations--an overview. Skin Pharmacol. Appl. Skin Physiol. 13, 229234, doi:29928 (2000).

112 Khiao In, M. et al. Histological and functional comparisons of four anatomical regions of porcine skin with human abdominal skin. Anatomia, histologia, embryologia 48, 207-217, doi:10.1111/ahe.12425 (2019).

113 Debeer, S. et al. Comparative histology and immunohistochemistry of porcine versus human skin. Eur. J. Dermatol. 23, 456-466, doi:10.1684/ejd.2013.2060 (2013).

114 Meurens, F., Summerfield, A., Nauwynck, H., Saif, L. \& Gerdts, V. The pig: a model for human infectious diseases. Trends Microbiol. 20, 50-57, doi:10.1016/j.tim.2011.11.002 (2012).

115 Levast, B., Schulz, S., Hurk, S. \& Gerdts, V. Animal models for neonatal diseases in humans. Vaccine 31, 24892499, doi:10.1016/j.vaccine.2012.11.089 (2013).

116 Nguyen, D. N. et al. Delayed development of systemic immunity in preterm pigs as a model for preterm infants. Scientific reports 6, 36816, doi:10.1038/srep36816 (2016).

117 Spengler, D., Rintz, N. \& Krause, M. F. An Unsettled Promise: The Newborn Piglet Model of Neonatal Acute Respiratory Distress Syndrome (NARDS). Physiologic Data and Systematic Review. Front. Physiol. 10, 1345, doi:10.3389/fphys.2019.01345 (2019).

118 Rose, N. \& Andraud, M. The use of vaccines to control pathogen spread in pig populations. Porcine health management 3, 8, doi:10.1186/s40813-017-0053-6 (2017).

119 van Dixhoorn, I. D. et al. Enriched Housing Reduces Disease Susceptibility to Co-Infection with Porcine Reproductive and Respiratory Virus (PRRSV) and Actinobacillus pleuropneumoniae (A. pleuropneumoniae) in Young Pigs. PloS one 11, e0161832, doi:10.1371/journal.pone.0161832 (2016).

120 Lee, I. K. et al. Stress, Nutrition, and Intestinal Immune Responses in Pigs - A Review. Asian-Australasian journal of animal sciences 29, 1075-1082, doi:10.5713/ajas.16.0118 (2016).

121 Amadori, M. \& Zanotti, C. Immunoprophylaxis in intensive farming systems: the way forward. Veterinary immunology and immunopathology 181, 2-9, doi:10.1016/j.vetimm.2016.02.011 (2016). 


\section{Appendix}

Abbreviations

Summary

Samenvatting

About the author

Dankwoord 


\section{Abbreviations}

Ag

Ab

ANOVA

APC

AUC

B-cell

BDC

BEI

BMDC

CCD

CCL

CCR

CD

CDC

CDNA

CPE

CPG ODN

CXCL

DC

DEFB1

DMN-patch

dpi

ELISA

ELISpot

FBS

FITC

FL

FLT3L

GM-CSF

HE

HEPA

i.m.

IF

IFN
Antigen

Antibody

Analysis of variance

Antigen presenting cell

Area under the curve

Bursa cell

Blood derived dendritic cell

Binary ethylenimine

Bone marrow derived dendritic cell

Dutch central authority for scientific procedures on animals

Chemokine ( $\mathrm{C}-\mathrm{C}$ motif, a-chemokine) ligand

Chemokine ( $\mathrm{C}-\mathrm{C}$ motif, a-chemokine) receptor

Cluster of differentiation

Conventional dendritic cells

Copy Desoxyribonucleïnezuur (copyDNA)

Cytopathologic effect

Cytosine phosphodiester Guanine oligodeoxynucleotides -TLR9 agonist

Chemokine (CXC-motif, $\beta$-chemokine) ligand

Dendritic cell

Beta defensin 1

Dissolving microneedle patch

Days post infection

Enzyme-linked immune sorbent assay

Enzyme-linked immune absorbent spot

Fetal bovine serum

Fluorescein isothiocyanate

Fetal liver

Growth factor fms-like tyrosine kinase 3 ligand

Granulocyte-macrophage colony-stimulating factor

Hematoxylin and eosin

High-efficiency particulate air

Intramuscular

Immunofluorescence

Interferon 


\begin{tabular}{|c|c|}
\hline Ig & Immunoglobulin \\
\hline IHC & Immunohistochemistry \\
\hline IL & Interleukin \\
\hline imTLRa & Intramuscular administered Toll-like receptor agonist \\
\hline IPMA & Immunoperoxidase monolayer assay \\
\hline iPRRSV & Inactivated porcine reproductive and respiratory syndrome virus \\
\hline IRF & Interferon regulatory factor \\
\hline ISA28 & Montanide $^{\mathrm{TM}}$ ISA28 (oil-in-water emulsion) \\
\hline LC & Langerhans cell \\
\hline LN & Lymph node \\
\hline MACS & Magnetic-activated cell sorting \\
\hline MARC-145 & Meat Animal Research Center-145 (non-human primate cell line) \\
\hline MARC-Sn & MARC-sialoadhesin \\
\hline MD2 & Lymphocyte antigen 96 \\
\hline MHC II & Major histocompatibility complex II \\
\hline Mo & Monocyte \\
\hline MoDC & Monocyte derived DC \\
\hline Mф & Macrophage \\
\hline mRNA & Messenger RNA \\
\hline MyD88 & Myeloid differentiation primary response 88 \\
\hline NF-кB & Nuclear factor kappa-light-chain-enhancer of activated B cells \\
\hline NK-cell & Natural killer cell \\
\hline NV & Non-vaccinated \\
\hline OD & Optical density \\
\hline O/W & Oil-in-water emulsion \\
\hline O/W con & Oil-in-water reference or control adjuvant -Montanide ${ }^{\mathrm{TM}}$ ISA28 \\
\hline PAM & Pulmonary alveolar macrophage \\
\hline Pam3Cys & Pam3Cys-SKKKK-TLR1/2 agonist \\
\hline PBMC & Peripheral blood mononuclear cells \\
\hline PBMC/CD3- & Enriched innate mononuclear cell population \\
\hline pDC & Plasmacytoid dendritic cells \\
\hline PDMS & polydimethylsiloxane \\
\hline PRR & Pattern recognition receptor \\
\hline PRRSV & Porcine reproductive and respiratory syndrome virus \\
\hline PRRSV-1 & Porcine reproductive and respiratory syndrome virus type- 1 \\
\hline PRRSV-2 & Porcine reproductive and respiratory syndrome virus type- 2 \\
\hline
\end{tabular}




\begin{tabular}{|c|c|}
\hline PVA & polyvinylalcohol \\
\hline qPCR & Quantitative polymerase chain reaction \\
\hline R848 & TLR7/8 agonist Resiquimod \\
\hline $\mathbf{R I N}$ & RNA integrity number \\
\hline RNA & Ribo Nucleic Acid \\
\hline RPMI medium & Roswell Park Memorial Institute \\
\hline RT-qPCR & Real time quantitative polymerase chain reaction \\
\hline SD & Standard deviation \\
\hline SE & Stable oil-in-water emulsion with squalene \\
\hline skiTLRa & Toll-like receptor agonist administered by skin vaccination \\
\hline SPF & Specific pathogen free \\
\hline SWE & Squalene-in-water emulsion \\
\hline T-cell & Thymus cell \\
\hline TCID $_{50}$ & Fifty-percent tissue culture infective dose \\
\hline Th & Thelper \\
\hline Th1/2 & T helper cell response type $1 / 2$ \\
\hline $\mathbf{T}_{\mathrm{FH}}$ & T follicular helper cell \\
\hline TGF- $\beta$ & Transforming growth factor beta \\
\hline TLR ligand & Toll-like receptor ligand \\
\hline TLRa & Toll-like receptor agonist \\
\hline TNF & Tumor necrosis factor alpha \\
\hline $\mathbf{T}_{\text {REG }}$ & T regulatory cell \\
\hline TRIF & TIR-domain-containing adapter-inducing interferon- $\beta$ \\
\hline
\end{tabular}




\section{Summary}

Young piglets are very susceptible to infections and therefore vaccination early after birth can help to decrease the disease incidence on swine farms. However, vaccination during the neonatal period is a challenge, because vaccination of newborn piglets is in general less successful compared to adult pigs. Before birth, the development of the specific (adaptive) immune system of the foetus is limited and for protection against infections newborns rely strongly on their non-specific (innate) immune system and the presence of maternal antibodies. The innate and adaptive immune system are still developing during early-life and this results in qualitative and quantitative differences between the immune responses of newborn or neonatal piglets $(<1$-week-old) and adult or immunocompetent ( $>$ 8-week-old) pigs after vaccination. Research has indicated that effective immuno-stimulators (adjuvants) in adult vaccines may not fulfil the requirements to stimulate the neonatal immune system effectively. Therefore, age-group-adapted vaccine formulations may be necessary to enhance the success of vaccination at an early age.

Toll-like receptors (TLRs) are one of the best studied family of pattern recognitions receptors (PRR) that are required to recognize and control pathogens, such as viruses, bacteria or fungi. Synthetic TLR agonists (TLRa) can also stimulate specific TLRs to induce an innate immune response e.g. by the induction of cytokine production. Therefore, synthetic TLR-agonists are considered powerful immuno-stimulators (adjuvants), which can be used in vaccines to enhance the immune response by stimulation and /or activation of antigen presenting cells (APCs). Activated APCs are an essential link between the innate and adaptive immune system, that is needed for successful vaccine responses. For this thesis we selected three different TLR agonist (TLR1/2a (Pam3Cys), TLR7/8a (R848) and TLR9a (CpG ODN)) based on their success in human and porcine studies to stimulate neonatal and adult immune cells.

The aim of this thesis was to investigate immune responses in neonatal and adult pigs afterTLR-stimulation to increase our understanding of the porcine (neonatal) immune system and the immune responses after vaccination in pigs of different age-groups. In addition to conventional intramuscular (i.m.) vaccination, skin vaccination (cutaneous vaccination) was used as a delivery route, because skin has the potential to increase vaccine efficacy due to its abundance of APCs. Porcine reproductive and respiratory syndrome virus (PRRSV) was selected as vaccine-antigen, because PRSSV is an endemic and important disease in the swine farming. In addition, to date no effective inactivated PRRSV-vaccine has been developed yet.

In chapter 2, we studied dendritic cells (DCs), the most important APC or so-called professional APC. We showed that neonatal porcine DCs derived from blood were composed of the same DC subsets and DC proportions as their adult counterparts, namely: plasmacytoid DCs (pDC), and two types of conventional DCs ( $\mathrm{DC} 1$ and $\mathrm{CDC2}$ ). After TLR1/2 or TLR9 stimulation peripheral blood mononuclear cells (PBMCs) and DCs 
were activated in both neonatal and adult pigs in an age-dependent way. With neonatal cells, TLR1/2a induced a stronger TNF expression in monocytes and $\mathrm{pDCs}$, and a stronger CD80/86 upregulation in CDC1 cells compared to adult cells. Also in neonatal cells, TLR9a was more potent at inducing IL12p40 mRNA expression, an important cytokine for the induction of antigen-specific cellular immune responses. The results of chapter 2 indicated that TLR1/2a and TLR9a could be promising adjuvant candidates in porcine vaccines for both age-classes.

In chapter 3, neonatal and adult pigs were vaccinated in the skin using dissolving microneedle (DMN)-patches as the delivery system. The DMN-patches contained three different individual TLR-agonist-vaccine formulations in combination with inactivated PRRSV (iPRRSV)-antigen. In this study, we investigated the early immune responses in skin and draining lymph nodes $24 \mathrm{~h}$ after skin vaccination. The DMN-patches containing TLR7/8a induced the most pronounced immune and/or inflammatory response in both age-groups. In neonatal pigs a higher number of genes related to early immune responses were upregulated after TLR7/8a vaccination compared to the adult pigs in both the skin and draining LN, while no age-dependent difference for the local influx of immune cells and the number of APCs in the skin after TLR7/8a vaccination was found. Less striking local vaccine immune responses were noticed also after TLR1/2a and TLR9a application. These results suggested that neonatal and adult pigs could be effectively vaccinated in the skin, especially with TLR7/8a containing vaccines. However, for the development of a skin vaccine, local and systemic age-dependent immune responses after skin vaccination should be considered.

In the adult PRRSV vaccine-study (chapter 4) we investigated if the specific individual TLR-agonists, which have shown in vitro potential (chapter 2 ) and induced early immune responses after skin vaccination (chapter 3), generated a protective immune response after vaccination in combination with iPRRSV-antigen. To study this, we administered the TLR-agonists containing vaccines both i.m. and into the skin with the same DMN-patches as used in chapter 3. Pigs received a prime vaccination followed by a booster vaccination four weeks later. To measure vaccine efficacy the pigs were infected with PRRSV three weeks after the booster vaccination. In adult pigs TLR1/2a, TLR7/8a or TLR9a containing PRRSVvaccines did not induce a detectable PRRSV-specific immune response, independent of the administration route. However, the i.m. administered TLR9a vaccine showed reduction of PRRSV shedding after infection compared to the non-vaccinated animals without detectable specific immune responses. In contrast, iPRRSV-antigen combined with the reference oil-in water (O/W) emulsion Montanide ${ }^{\mathrm{TM}}$ ISA28 (ISA28) induced an antigenspecific humoral immune response after booster vaccination combined with reduced shedding of PRRSV. A local skin immune response was observed after skin application of TLR7/8a. This skin reaction was comparable to that observed in adult pigs in chapter and 
was not observed in the other TLR-agonist skin vaccines. These results indicated that the individual TLR-agonists were less effective in generating a specific-immune response than the $\mathrm{O} / \mathrm{W}$ reference adjuvant ISA28.

We considered it unlikely that the same TLR-agonist containing vaccine would be more effective when administered in neonatal piglets. Therefore, in chapter $\mathbf{5}$ we decided to use different adjuvant formulations for the neonatal PRRSV-vaccine-study. In this study a mixture of the previously used TLR-agonists (TLR1/2a+TLR7/8a+TLR9a) was combined with a squalene based O/W emulsion (SWE) and this vaccine formulation (SWE+TLRa) was administered i.m and into the skin with DMN-patches using a study design comparable to the adult PRRSV-study. The reference O/W adjuvant ISA28 was used in both the neonatal and adult PRRSV-vaccine study. In this neonatal study, only ISA28 induced a specific humoral immune response, while a specific cellular immune response was generated in the SWE group. This suggests that prime vaccination in neonates induced a specific immune response after booster vaccination, dependent on the $\mathrm{O} / \mathrm{W}$ emulsion formulation, but not dependent on the presence of the TLR-agonists or delivery route. In contrast to the adult pigs none of the vaccines in neonatal piglets with or without TLR-agonists were able to reduce the viral shedding after PRRSV challenge. This study demonstrated that the O/W adjuvant ISA28 was less effective in neonatal piglets compared to adult pigs and that the TLR-agonists in an adjuvant-mixture did not enhance the immune response in a PRRSV vaccine

In chapter $\mathbf{6}$ I reviewed the thesis results by addressing three major research questions: (1) Are the immune responses after TLR stimulation or PRRSV vaccination age-dependent?; (2) Are TLR agonists able to enhance the specific immune response of the PRRSV vaccine? ; (3) Does skin administration of the PRRSV vaccine provide an enhanced immune response compared to conventional i.m. vaccination? Based on the data obtained I conclude that age-dependent differential immune responses could be shown after in vitro (chapter 2) and in vivo TLR stimulation (chapter $\mathbf{3}$ ) and also by using O/W adjuvanted PRRSV vaccination (chapter 4 and 5). This suggests that neonatal vaccines require a specific adjuvant choice. Further, I conclude that traditional $\mathrm{O} / \mathrm{W}$ formulations induced a better detectable immune response than when the TLR-agonists were used as adjuvant in combination with the iPRRSVantigen. Also, we showed that skin vaccination with TLR-agonists did not overcome the weak immunogenic potential of the iPRRSV-antigen (chapter 4 and 5). Therefore, the administered vaccine formulations with TLR-agonists were less successful as expected and it can be anticipated that formulating an effective iPRRSV vaccine for (newborn) pigs will continue to be a challenge. 


\section{Samenvatting}

Jonge biggen zijn vatbaar voor infecties en daarom kan vaccinatie vroeg na de geboorte (de neonatale periode) helpen om op varkensbedrijven het optreden van ziektes te verminderen en de ziekteduur te verkorten. Vaccinatie tijdens deze neonatale periode is echter een uitdaging omdat het afweersysteem (immuunsysteem) nog volop in ontwikkeling is in deze vroege levensfase. Daarom is vaccinatie van pasgeboren biggen over het algemeen minder succesvol dan vaccinatie van volwassen varken. Voor de geboorte is de ontwikkeling van het specifieke immuunsysteem (Box 1$)$ in de foetus beperkt en voor bescherming tegen infecties vertrouwen pasgeborenen dus heel sterk op hun niet-specifieke (aangeboren) immuunsysteem en de aanwezigheid van maternale antilichamen.

\section{Box 1.}

\section{Het immuunsysteem: Niet-specifieke (innate) en specifieke (adaptieve) afweer}

De niet-specifieke afweer wordt gevormd door barrières (huid en slijmvliezen), die zich niet richten tegen een specifieke ziekteverwekker, maar deze wel buitensluiten om te voorkomen dat een organisme wordt geïnfecteerd (eerste lijn). Daar bovenop (tweede lijn) staat de aangeboren afweer (innate immuniteit), die altijd en overal aanwezig is en klaarligt om met behulp van specifieke stoffen en cellen ziekteverwekkers te herkennen en te verwijderen. Deze vorm van afweer werkt snel (binnen enkele minuten), maar er wordt geen langdurig geheugen opgebouwd.

Voor een goede afweerreactie is de hulp van de specifieke afweer nodig, die meer tijd (enkele uren tot dagen) nodig heeft om zich te ontwikkelen en zich optimaal aan te passen aan de verwijdering van de ziekteverwekker (adaptieve immuniteit). Hierbij worden vele en krachtige afweercellen en antistoffen gevormd, die specifiek werken tegen de betreffende ziekteverwekker. Na herstel wordt er een immunologisch geheugen opgebouwd in de vorm van en specifieke afweercellen en antilichamen, die bij herinfectie een snelle bescherming geven. De opbouw van deze specifieke afweer is het belangrijkste doel van een goede vaccinatie. Echter, de aangeboren afweer, die direct ontstaat na het toedienen van de vaccinatie, kan de opbouw van de vaccinatiespecifieke immuunreactie ondersteunen. Daarom kan de aangeboren afweer dus ook belangrijk bijdrage leveren aan een effectieve vaccinatie.

Na vaccinatie resulteert dit verschil in ontwikkeling van het immuunsysteem in kwalitatieve en kwantitatieve verschillen in immuunreacties tussen pasgeboren of neonatale biggen ( $<1$ week oud) en volwassen of immuun competente ( $>8$ weken oude) varkens. Uit eerder onderzoek blijkt, dat immuunstimulatoren (adjuvantia) in vaccins (Box 2) voor volwassen dieren het neonatale immuunsysteem mogelijk onvoldoende stimuleren. Daarom kunnen op diverse leeftijdsgroepen aangepaste vaccinatie formuleringen ook het succes van vaccinatie op jonge leeftijd vergroten. 


\section{Box 2.}

\section{Hulpstoffen in vaccins: Adjuvantia}

Het belangrijkste onderdeel in het vaccin is het antigeen, de ziekteverwekker, waartegen de vaccinatie in de toekomst moet beschermen door de inductie van een krachtige immuunreactie. Vaak wordt uit veiligheidsoverwegingen de ziekteverwekker in een verzwakte vorm (geïnactiveerd) in het vaccin gestopt, zodat er na vaccinatie geen ziekte kan optreden. Bij zo'n geïnactiveerd vaccin is dan een hulpstof nodig (adjuvans) om de opbouw van de immuunreactie te stimuleren. Adjuvantia veroorzaken over het algemeen een aangeboren (innate) immuunreactie, die de opbouw van de specifieke immuunreactie van het betreffende antigeen ondersteunt.

Toll-like receptoren (TLR) (Box 3) zijn aanwezig op een groot aantal typen immuuncellen en herkennen ziekteverwekkers (pathogenen), zoals virussen, bacteriën of schimmels. $\mathrm{Na}$ TLR-activatie door een pathogeen komt er een aangeboren immuunreactie op gang, bijvoorbeeld middels productie van diverse cytokines, die helpt bij de bestrijding van de betreffende pathogenen. Synthetische TLR-agonisten kunnen ook specifieke TLR's stimuleren en zijn de afgelopen jaren uitgebreid onderzocht voor mogelijke toepassing als adjuvans in vaccins. Voor dit proefschrift selecteerden we drie verschillende synthetische TLR-agonisten (TLR1/2a (Pam3Cys), TLR7/8a (R848) en TLR9a (CpG ODN)) op basis van humane- en varkensstudies, waarin neonatale en volwassen immuuncellen succesvol werden gestimuleerd door deze TLR-agonisten.

\section{Box 3.}

\section{Toll-like receptor-agonist}

Toll-like receptoren zijn ontvangers op of in de cel, die delen van ziektekiemen herkennen en hiermee belangrijk zijn voor het in gang zetten van de aangeboren of innate immuunreacties. Er zijn meerdere TLR (1-10) in het varken, die vaak specifieke delen van ziektekiemen herkennen, bijvoorbeeld het genetisch materiaal in een virus, de celwand van een bacterie of een schimmel. Kunstmatig gemaakte stoffen kunnen ook specifieke typen TLR op of in de cel stimuleren om zo een vergelijkbare reactie op te wekken als na stimulatie door een ziektekiem. Deze kunstmatige stoffen worden TLR-agonisten genoemd en kunnen worden gebruikt als hulpstoffen (adjuvantia) in een vaccin om de immuunreactie te verhogen na vaccinatie.

Door stimulatie en/of activatie van antigeen presenterende cellen (APC's) (Box 4) kunnen TLR-agonisten de immuunreactie na vaccinatie versterken. Geactiveerde APC's zijn een essentiële schakel tussen de aangeboren en adaptieve hoog-specifieke immuunreactie en deze schakel is belangrijk voor een succesvolle immuunrespons na vaccinatie. 


\section{Box 4.}

\section{Antigeen presenterende cellen (APC's)}

Meerdere soorten immuuncellen kunnen het antigeen (bijvoorbeeld de ziekteverwekker) of een deel daarvan presenteren aan andere immuuncellen, zodat via deze verbinding de specifieke afweerreactie kan worden gestart. Dit type immuuncellen wordt antigeen presenterende cellen (APC) genoemd. Grote aantallen APC's bevinden zich in (de nabijheid van) slijmvlies (bijvoorbeeld in de darm of neus) en in de huid, maar ook een kleine fractie van de witte bloedcellen fungeert als APC en ook de milt en lymfeknopen bevatten APC's. In tegenstelling tot macrofagen en B-cellen, die naast het presenteren van antigeen ook belangrijke andere taken hebben in de afweerreactie, is de hoofdtaak van dendritische cellen (DC) het presenteren van antigeen. DC's worden daarom ook vaak professionele APC's genoemd. Deze cellen hebben lange tentakels (dendrieten), die het antigeen opvangen, onder andere met hun TLR's. Vervolgens breken ze de antigenen af en presenteren onderdelen aan andere immuuncellen zoals T-cellen.

Het doel van dit proefschrift was om de opbouw en functie van het immuunsysteem en de rol in vaccinatie reacties bij pasgeboren biggen te onderzoeken na toepassing van synthetische TLR-agonisten en dit te vergelijken met volwassen varkens. Met deze resultaten willen we het begrip van de immuunreacties na vaccinatie vergroten in met name pasgeboren biggen om zo verbeterde vaccins voor diverse leeftijdsgroepen te kunnen ontwikkelen. Naast de gebruikelijke intramusculaire (i.m.) vaccinatie, werd de huid gebruikt als een alternatieve vaccinatieroute (transcutane vaccinatie). De huid bevat een groot aantal APC's en kan als toedieningsroute mogelijk de werkzaamheid van het vaccin verhogen. Het porcine reproductive and respiratory syndroom virus (PRRSV) werd geselecteerd als vaccin-antigeen, omdat PRRSV een veel voorkomend en lastig te bestrijden virus is in de varkenshouderij. Bovendien is er tot op heden nog geen effectief geïnactiveerd PRRSV-vaccin beschikbaar.

In hoofdstuk 2 hebben we dendritische cellen (DC's), de belangrijkste APC of zogenaamde professionele APC in vitro bestudeerd. We toonden aan dat het bloed van neonatale en volwassen varkens overeenkomstige typen DC's bevat en in een onderling vergelijkbare verhouding. De belangrijkste typen DC's waren plasmacytoïde DC's (pDC) en twee soorten conventionele DC's (cDC1 en cDC2). Na stimulatie met TLR1/2 of TLR9- agonisten werden de perifere mononucleaire bloedcellen (PBMC's) en DC's geactiveerd in het bloed van neonatale biggen en volwassen varkens. Echter, er waren duidelijke verschillen tussen de leeftijdsgroepen. In de neonatale cellen veroorzaakte TLR1/2 stimulatie een sterkere expressie van het cytokine TNF in monocyten en pDC's, en tevens een hogere expressie van CD80/86 op cDC1-cellen in vergelijking met dezelfde cellen uit het bloed van volwassen varkens. Ook in neonatale cellen verhoogde de TLR9 activatie meer mRNA-expressie van 
het cytokine IL12p40 in verhouding tot volwassen cellen, dit is een belangrijk cytokine voor de aansturing van specifieke cellulaire immuunreacties. De resultaten van hoofdstuk 2 laten zien dat TLR1/2 en TLR9-agonisten mogelijk veelbelovende adjuvans kandidaten zijn voor vaccins voor varkens in beide leeftijdsklassen.

In hoofdstuk 3 werden neonatale en volwassen varkens in de huid (transcutaan) gevaccineerd met oplossende micro-naalden (dissolving microneedle (DMN)-pleisters). Deze DMN-pleisters bevatten steeds één van de drie verschillende TLR-agonisten in combinatie met geïnactiveerd PRRSV als antigeen. In deze studie onderzochten we de vroege immuunreacties in de huid en drainerende lymfeknopen 24 uur na vaccinatie in de huid. De DMN-pleisters, die TLR7/8-agonisten bevatten, veroorzaakten in beide leeftijdsgroepen de meest duidelijke immuun- en/of ontstekingsreactie. In neonatale biggen werden in een TLR7/8 gestimuleerde vaccinatie respons meer immunogenen gereguleerd in vergelijking met de volwassen varkens, in zowel de huid als de drainerende lymfeknoop. Echter, we vonden geen leeftijdsafhankelijk verschil voor de lokale immuunreactie (in histologische analyse) en het aantal APC's in de huid (met immunohistochemische kleuringen) na vaccinatie gebruikmakend van TLR7/8 stimulatie. Huidvaccinatie met TLR1/2 en TLR9-agonisten genereerde minder duidelijke immuunreacties. Deze studie laat zien dat neonatale en volwassen varkens effectief in de huid kunnen worden gevaccineerd en dat met name met TLR7/8-agonist bevattende vaccins veelbelovend zijn. Echter, bij het ontwikkelen van vaccins voor in de huid, moet men rekening houden met lokale en systemische leeftijdsafhankelijke immuunreacties.

In de PRRSV-vaccinatiestudie bij volwassenen varkens (hoofdstuk 4) hebben we onderzocht of de individuele TLR-agonisten, die in vitro effectief waren (hoofdstuk 2) en vroege immuunreacties veroorzaakten na huidvaccinatie (hoofdstuk 3), ook in vivo in staat waren om een beschermende immuunreactie te genereren na vaccinatie. Om dit te onderzoeken hebben we de TLR-agonist bevattende vaccins i.m. en in de huid (transcutaan) aangebracht, waarbij we voor de huidvaccinatie dezelfde DMN-pleisters gebruikten als in hoofdstuk 3. De varkens kregen een eerste vaccinatie gevolgd door een herhalingsvaccinatie vier weken later. Om het beschermend effect na vaccinatie te meten, werden de varkens drie weken na de herhalingsvaccinatie geïnfecteerd met PRRSV. Bij volwassen varkens werd na vaccinatie met een PRRSV-vaccin met TLR1/2, TLR7/8 of TLR9agonisten geen PRRSV-specifieke immuunreactie waargenomen en dit was onafhankelijk van de toedieningsroute. Alleen huidvaccinatie met TLR7/8-agonist veroorzaakte een duidelijke lokale huidreactie, die vergelijkbaar was met de TLR7/8-agonist huidreactie in hoofdstuk 3. Het is een interessante bevinding, dat varkens met het i.m. toegediende TLR9agonist bevattende vaccin een afname van de PRRSV-uitscheiding na infectie vertoonden in vergelijking met de niet-gevaccineerde dieren. Het referentie vaccin (positieve controle vaccine), geïnactiveerd PRRSV in combinatie met de referentie olie-in-water (O/W)- 
emulsie Montanide ${ }^{\mathrm{TM}}$ ISA28 (ISA28), veroorzaakte daarentegen de vorming van een PRRSV-specifieke antilichaam reactie na herhalingsvaccinatie en tevens een verminderde PRRSV-uitscheiding na infectie. Deze resultaten tonen aan dat met betrekking tot de specifieke antistofvorming, de individuele TLR-agonisten minder effectief zijn dan het O/W-referentie-adjuvans ISA28.

We achtten het onwaarschijnlijk dat TLR-agonist bevattende vaccins gebruikt voor de volwassen varkens effectiever zouden zijn in neonatale biggen. Daarom besloten we om voor de neonatale PRRSV-vaccinatie studie in hoofdstuk 5 nieuwe adjuvans formuleringen te gebruiken. In deze studie werd een mengsel van de eerder gebruikte TLRagonisten (TLR1/2a + TLR7/8a + TLR9a) gecombineerd met een op squaleen gebaseerde O/W-emulsie (SWE) en deze vaccinformulering (SWE + TLRa) werd i.m. en in de huid met DMN-pleisters toegediend in een onderzoeksopzet vergelijkbaar met de PRRSV-studie bij volwassenen varkens in hoofdstuk 4. Het referentie-O/W-adjuvans ISA28 werd zowel in de PRRSV-vaccinatie studie bij neonatale als bij volwassen varkens gebruikt. In deze neonatale vaccinatie studie veroorzaakten alleen de $\mathrm{O} / \mathrm{W}$ emulsies een specifieke immuun reactie: ISA28 een PRRSV-specifieke antilichaam reactie en SWE zonder TLR-agonisten een specifieke cellulaire immuunreactie. Dit suggereert, dat de specifieke immuunreactie, die ontstaat na eerste vaccinatie in neonatale biggen gevolgd door een herhalingsvaccinatie, afhankelijk is van het type O/W-emulsie, maar niet afhankelijk is van de aanwezigheid van TLR-agonisten of toedieningsroute. In tegenstelling tot de volwassen varkens was geen van de vaccinformuleringen bij de neonatale biggen met of zonder TLR-agonisten in staat om de virusuitscheiding na PRRSV infectie te verminderen. Deze studie toont aan dat het O/W-emulsie ISA28 minder effectief is bij neonatale biggen in vergelijking tot volwassen varkens en dat de TLR-agonisten in een adjuvansmengsel de immuunrespons bij een PRRSV-vaccin niet versterken.

In hoofdstuk 6 heb ik de resultaten van het proefschrift besproken aan de hand van drie belangrijke onderzoeksvragen: (1) Zijn de immuunreacties na TLR-stimulatie of PRRSVvaccinatie leeftijdsafhankelijk?; (2) Kunnen TLR-agonisten de specifieke immuunrespons van het PRRSV-vaccin versterken?; (3) Levert de toediening van het PRRSV-vaccin via de huid een verbeterde immuunrespons op in vergelijking met conventionele i.m. vaccinatie? Voor vraag (1) laat dit proefschrift leeftijdsafhankelijke immuunreacties zien na in vitro (hoofdstuk 2) en in vivo TLR-stimulatie (hoofdstuk 3) en ook na vaccinatie met O/W-emulsie ISA28 in combinatie met geïnactiveerd PRRSV (hoofdstuk 4 en 5). Dit suggereert dat neonatale vaccinatie een specifieke adjuvans keuze vereist. Voor vraag (2) concludeer ik, dat in dit proefschrift vaccinformuleringen met traditionele O/W-emulsies een beter detecteerbare immuunreactie genereren, dan wanneer de TLR-agonisten als adjuvans worden gebruikt in combinatie met het geïnactiveerde PRRSV-antigeen. Ook tonen we aan voor vraag (3), dat huidvaccinatie met TLR-agonisten de zwakke immunogene potentie van 
geïnactiveerd PRRSV niet overwint (hoofdstuk 4 en 5) in vergelijking tot i.m. vaccinatie. Mogelijk door de zwakke immuun eigenschappen van het geïnactiveerde PRRSV-antigeen waren de toegediende vaccinaties met TLR-agonisten minder succesvol dan ingeschat. Mede hierom zal het ontwikkelen van een succesvol geïnactiveerd PRRSV-vaccin voor (pasgeboren) varkens een uitdaging blijven. 


\section{About the author \\ Curriculum Vitae}

Sandra was born on July 23, 1973 in SantpoortNoord as a baker's daughter. From an early age she enjoyed helping in the bakery and occasionally you can still find her in bakery "Ben Vreman", which has now been taken over by her brother. Already in primary school she knew exactly what she wanted to become: a vet. In 1991 she started her education at the veterinary faculty of Ghent and continued a year later at the veterinary faculty of Utrecht.

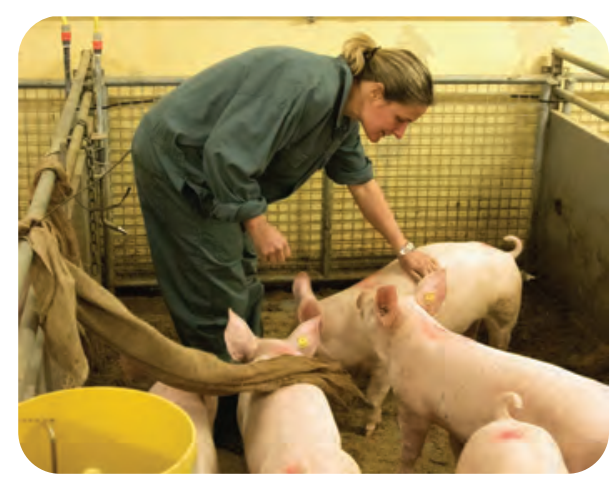
While studying veterinary medicine, there was always close contact with the home front because of the work in the bakery, combined with the tennis training / competition for the local tennis club. During the waiting period for the internships, she participated in an Erasmus exchange to conduct an anaesthesiology study at the Veterinary University in Thessaloniki (Greece). In early 2020 she obtained the degree of Doctor of Veterinary Medicine. For more than 7 years, she worked in several pet clinics with a special focus on feline medicine and small rodents. During this period, the bond with the "Poezenboot" in Amsterdam developed, where she still likes to be found. The diploma of tennis teacher was also added to extend the tennis hobby by giving tennis lessons. In this time it became apparent that the work as a small animal veterinarian was not satisfactory and a trip was made outside of veterinary medicine.

From 2007 Sandra supported human oncology research in various hospitals as a clinical trial monitor for Glaxo Smith Kline. In early 2010, Sandra followed her heart by starting a training as a veterinary pathologist at Utrecht University. An intensive training program, but the feeling that your work is your hobby, accompanied by a great group of colleagues, made this an enjoyable and memorable period. After obtaining the diploma of the European College of Veterinary Pathologists (ECVP), she worked for a short period as a veterinary pathologist at the Utrecht University. At the end of 2015, a new challenge was started at Wageningen Bioveterinary Research (WBVR) with a PhD focussed on immunopathology. This PhD was in collaboration with the cell biology and immunology (CBI) group of the Wageningen University and with partners of a large EU-project called SAPHIR (Strengthening Animal Production and Health through the Immune Response). In this period Sandra expanded her immunological knowledge and skills in Lelystad/ Wageningen, but also outside the Netherland, in Bern, London and Copenhagen, together with partners from the SAPHIR-project.

She currently works as a veterinary pathologist and researcher at the WBVR with special attention for immunopathology. Sandra lives with her partner Guy and cat Bella in Soesterberg, the Netherlands. 


\section{Levensloop werk}

Sandra werd geboren op 23 juli 1973 in Santpoort-Noord als bakkersdochter. Van jongs af aan heeft ze met veel plezier meegeholpen in de bakkerij en af en toe kun je haar nog steeds vinden in bakkerij "Ben Vreman", die nu overgenomen is door haar broer. Al op de basisschool wist ze precies wat ze wilde worden: dierenarts. In 1991 begon ze haar opleiding aan de veterinaire faculteit van Gent om een jaar later de studie te vervolgen

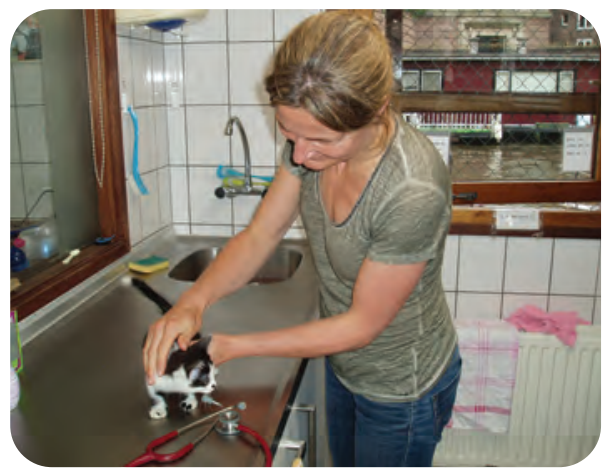
aan de veterinaire faculteit van Utrecht. Tijdens de studie diergeneeskunde was er altijd nauw contact met het thuisfront vanwege het werk in de bakkerij, gecombineerd met de tennistraining/ competitie voor de lokale tennisclub. Tijdens de wachttijd voor haar coschappen nam ze deel aan een Erasmus uitwisseling om anesthesiologie onderzoek uit te voeren aan de Veterinaire Universiteit in Thessaloniki (Griekenland). Begin 2020 studeerde ze af als dierenarts. Gedurende ruim 7 jaar werkte ze in verschillende klinieken voor gezelschapsdieren met speciale interesse voor katten en kleine knaagdieren. In deze periode ontstond de band met de "Poezenboot" in Amsterdam, waar ze nog steeds graag te vinden is. Tevens werd het diploma tennisleraar behaald om de tennishobby uit te breiden met het geven van tennislessen. Na verloop van tijd bleek, dat het werk als gezelschapsdierenarts onvoldoende voldoening gaf en er werd een uitstapje gemaakt buiten de diergeneeskunde.

Vanaf 2007 ondersteunde Sandra humaan oncologisch onderzoek in verschillende ziekenhuizen als clinical trial monitor voor Glaxo Smith Kline. Begin 2010 besloot Sandra haar hart te volgen, door een opleiding tot veterinair patholoog aan de Universiteit Utrecht te starten. Een intensief trainingsprogramma, maar het gevoel dat je werk je hobby is, vergezeld van een geweldige groep collega's, maakte dit een plezierige en gedenkwaardige periode. Na het behalen van het diploma voor het Europese College van of Veterinaire Pathologie (ECVP) werkte zij een korte tijd als veterinair patholoog aan de Universiteit Utrecht. Eind 2015 startte ze een nieuwe uitdaging bij Wageningen Bioveterinary Research (WBVR) met een promotieonderzoek (PhD). Dit PhD was in samenwerking met de celbiologie en immunologie $(\mathrm{CBI})$ groep van de Wageningen Universiteit en met partners uit het EU-project genaamd SAPHIR (Strengthening Animal Production and Health through the Immune Response). In deze periode kreeg Sandra de kans om haar immunologische kennis en vaardigheden uit te breiden in Lelystad/ Wageningen, maar ook buiten Nederland, in Bern, London en Kopenhagen, bij partners uit het SAPHIR-project

Op dit moment werkt zij als veterinair-patholoog en onderzoeker bij WBVR met aandacht voor de immunopathologie. Sandra woont samen met haar partner Guy en kat Bella in Soesterberg. 


\section{List of publications}

\section{This thesis}

Sandra Vreman, Joanne McCaffrey, Ditta J. Popma-de Graaf, Hans Nauwynck, Huub F.J. Savelkoul, Anne Moore, Johanna M.J. Rebel, Norbert Stockhofe-Zurwieden; Toll-Like receptor agonists as adjuvants for inactivated porcine reproductive and respiratory syndrome virus (PRRSV) vaccine. Veterinary. Immunology and Immunopathology (2019) June; 212; 27-37. https://doi.org/10.1016/j.vetimm.2019.04.008

Sandra Vreman, Gael Auray, Huub F.J. Savelkoul, Annemarie Rebel, Artur Summerfield, Norbert Stockhofe-Zurwieden; Neonatal porcine blood derived dendritic cell subsets show activation after TLR2 or TLR9 stimulation. Developmental and Comparative Immunology (2018) Jul;84:361-370. https://doi.org/10.1016/j.dci.2018.03.012

Sandra Vreman, Norbert Stockhofe-Zurwieden, Ditta J. Popma-de Graaf, Huub F.J. Savelkoul, C. Barnier-Quer, N. Collin, Damien Collins, Dennis McDaid, Anne C. Moore and Johanna M.J. Rebel; Immune responses induced by inactivated porcine reproductive and respiratory syndrome Virus (PRRSV) vaccine in neonatal pigs using different adjuvants. Veterinary immunology and Immunopathology, submitted (2019).

Sandra Vreman, Johanna M.J. Rebel, Joanne McCaffrey, Kristina Ledl, Ksenia Arkhipova, Damien Collins, Dennis McDaid, Huub F.J. Savelkoul, Kerstin Skovgaard, Anne C. Moore and Norbert Stockhofe-Zurwieden; Early immune responses in skin and lymph node after skin vaccination in neonatal and adult pigs. Vaccine, submitted (2020).

\section{Other publications}

Molenaar RJ, Vreman S, Hakze-van der Honing RW, Zwart R, de Rond J, Weesendorp E, Smit LAM, Koopmans M, Bouwstra R, Stegeman A, van der Poel WHM; Clinical and Pathological Findings in SARS-CoV-2 Disease Outbreaks in Farmed Mink (Neovison vison). Veterinary Pathology (2020) Jul. https://doi.org/10.1177/0300985820943535

Oreshkova Nadia, Molenaar Robert Jan, Vreman S. et al.; SARS-CoV-2 infection in farmed minks, the Netherlands, April and May 2020. Euro Surveillance (2020);25(23).

https://doi.org/10.2807/1560-7917.ES.2020.25.23.2001005

Vreman, S., Caliskan, N., Harders, F. et al.; Two novel porcine teschovirus strains as the causative agents of encephalomyelitis in the Netherlands. BMC Veterinary Research (2020) 16, 51 https://doi.org/10.1186/s12917-020-2275-0 
Otte CM, Valtolina C, Vreman S, Hubers S, van Wolferen ME, Favier RP, Rothuizen J, Penning LC; Immunohistochemical evaluation of the activation of hepatic progenitor cells and their niche in feline lymphocytic cholangitis. Journal of Feline Medicine and Surgery (2018) Jan;20(1):30-37. https://doi.org/10.1177/1098612x14520811

Otte CM, Rothuizen J, Favier RP, Penning LC, Vreman S; A morphological and immunohistochemical study of the effects of prednisolone or ursodeoxycholic acid on liver histology in feline lymphocytic cholangitis. Journal of Feline Medicine and Surgery (2014) Oct;16(10). https://doi.org/10.1177\%2F1098612X14520811

Vreman S, Wiemer $\mathrm{P}$, Keesler Rl; Bleeding in the subarachnoid space: a possible complication during laser therapy for equine progressive ethmoid haematoma. Tijdschrift voor Diergeneeskunde (2013) Oct 1;138(10):30-3.

https://pubmed.ncbi.n/m.nih.gov/24199337/

ORCID ID Sandra Vreman: https://orcid.org/0000-0003-0779-6251 


\section{Overview of completed training activities \\ According to European Credit Transfer System (ECTS)}

The Basic Package

2 ECTS

WIAS Introduction Day (mandatory)

2015

Course on philosophy of science and/or ethics (mandatory)

2015

Disciplinary Competences

16 ECTS

Basic Statistics R

2015

European Veterinary Vaccinology Workshop 2016

Advanced course immunology UMC 2016

BD multicolour FACS course $\quad 2016$

Research proposal WIAS 2016

CL Davis histology lymph node and lymphoma 2017

Kwaliteitsanalyse van real-time PCR amplificatiecurven 2018

WBVR course Study design and analysis of animal experiments 2018

Introductory Biostatistics UMCU 2018

Professional Competences

8 ECTS

Competence Assessment (WIAS) 2015

The Essentials of Scientific Writing \& Presenting 2016

Information Literacy including EndNote Introduction 2016

$\begin{array}{ll}\text { Scientific writing } & 2017\end{array}$

Organisation of SAPHIR annual meeting Lelystad $2018 \quad 2017$

Brain friendly working and writing 2018

Advanced communication in English C1-C2 2019

The final touch: writing general introduction and discussion 2019

Reviewing a Scientific Paper 2019

Societal impact of your research 2019

Writing propositions for your PhD 2019

Last stretch of PhD programme 2019

Presentation skills 4 ECTS

European College of Veterinary Pathology Congress (ECVP), Helsinki (oral) 2015

EU-project meeting Strengthening Animal Production and Health through 2016-2019

the Immune Response (SAPHIR) (oral)

WIAS science day (oral and poster) 2017-2018

Skin Vaccination Summit, Leiden (oral and poster) 2017

CVP, Lyon (oral and poster) 2017

European Veterinary Immunology Workshop (EVIW) Utrecht (poster) 2018 
ECVP, Arnhem (oral and poster)

2019

International Veterinary Immunology Symposium, Seattle (poster)

2019

Teaching competences

4 ECTS

Supervision bachelor thesis ( $2 \mathrm{x}$ )

2016-2017

Master class vaccine development

2018

Supervision master thesis

2019

Lecture porcine veterinarians (survival meeting 15mar19)

2019

Total

34 ECTS 


\section{Dankwoord}

$\mathrm{Na}$ een kleine vertraging door de COVID-19 pandemie, ben ik aangekomen bij het sluitstuk van mijn proefschrift. Vele lieve collega's, vrienden, familie en varkens hebben mij geholpen om dit prachtige boekje te maken en mezelf te ontwikkelen. Niemand vormt zichzelf alleen.

\section{Promotor}

Geachte Prof. Dr. H.J. Savelkoul, beste Huub, jouw betrokkenheid, enthousiasme en alomvattende immunologische kennis werken aanstekelijk. Bedankt voor je wijze woorden en suggesties voor het schrijven. Mijn passie voor pathologie is uitgebreid met die voor immunologie. Ik hoop dat ik in de toekomst nog eens mag aankloppen voor een privé-college.

"The more you know, the more you know you don't know"

Aristotle

\section{Copromotoren}

Geachte Prof. Dr. A. Rebel, beste Annemarie, je bent een persoon om op te bouwen. Een soms eigenwijze PhD student begeleiden is een uitdaging. Je begreep of zag aan mij, wanneer ik een luisterend oor nodig had. In de laatste fase van mijn proefschrift, moest er in een korte periode veel werk worden verzet. Voor jou was dit vanzelfsprekend. Bedankt voor je vertrouwen, punctualiteit en fijne gesprekken.

Geachte Dr. N. Stockhofe-Zurwieden, beste Norbert, jij bent een duizend poot die nooit stil zit: pathologie, immunologie, diermodellen, IvD en acquisitie. Helaas was de afgelopen periode niet eenvoudig voor jou en je gezin. Ik bewonder de kracht waarmee jij doorgaat en de manier waarop je je inzet voor de pathologie op ons instituut. Bedankt voor je positiviteit, vertrouwen en hulp bij het maken van dit proefschrift. Ik hoop de komende jaren nog veel van je te kunnen leren.

\section{Thesis committee}

I would like to thank, Prof. Andrea Gröne, Prof. Wim van de Poel, Prof. Victor Rutten and Dr Isabelle Schwartz for their evaluation of this thesis.

\section{Paranimfen}

Lieve Hanneke, na onze eerste ontmoeting in Gent ben je voor mij altijd de vriendin geweest om op te bouwen. Beiden zijn we een beetje 'stille wateren', maar als het er echt op aan komt ben je een rots in de branding. Bedankt voor je vriendschap, waarmee je mij het gevoel geeft er nooit alleen voor te staan. 
Lieve Ditta, wat had ik zonder jou gemoeten. Het was een hele klus om het PRRSV vaccin te produceren voor de dierstudies. Jij ging onverschrokken door, ondanks de strakke deadlines, en altijd met een glimlach. Je hielp mee in de stallen en met de analyse van de monsters. Als ik wat gespannen was voor een belangrijk momenten in de proef, dan zorgde jouw aanwezigheid voor ontspanning. Bedankt voor de fijne samenwerking!

\section{Pathologie groep infectie biologie}

Over deze groep kan ik een boek schrijven. Maar zonder gekheid: Pieter, Ralph, Rob, Corry, Lisette, Norbert, Lucien en Sebastiaan, jullie zijn collega's die bijna al het werk aangenaam maken. Pieter en Ralph, bedankt voor jullie hulp bij het afronden van de vaccinatie studies. Dit waren zware dagen, voor jullie met name fysiek: de varkens groeiden als kool. Voor mij met name emotioneel, want elk afscheid is lastig. Maar daarnaast heb ik enorm genoten van onze hardloop rondjes en onverwachte pathologie kadootjes uit de sectiezaal.

Corry en Lisette, de laboratorium tijgers. Jullie verwerken met groot gemak stapels monsters. Bedankt voor jullie hulp, want alleen had ik dit onmogelijk gekund. Maar zeker ook bedankt voor de gezelligheid en kletspraatjes, die ik zeker nodig had om mijn soms wat serieuze ondertoon af te vlakken. Lisette, ik nam me steeds voor om me de volgende keer beter te beheersen, maar jouw snoeppot op onze kamer gaf mij weer energie aan het einde van de dag. Bedankt voor het delen. Rob, bedankt voor jouw vriendschap en voor het delen van jouw kennis over immuunhistochemie, flow cytometrie en de natuur. Ik bewonder het gemak waarmee jij planten, bomen, vogels en insecten determineert.

\section{Dierverzorging en Biotechniek groep}

Bedankt voor de goede zorgen voor de varkentjes en de punctuele praktische uitvoering van de twee vaccinatie studies in dit proefschrift, die beiden maar liefst 70 dagen duurden. Er is ongelofelijk veel werk verzet in de stallen op de Runderweg en Houtribweg. Ik kan hier een tweede boek over schrijven. Dit waren intensieve periodes, want in een dierstudie mag je niets aan het toeval overlaten. Bijna dagelijks was ik in de stallen te vinden en dan zullen jullie wel eens hebben gedacht: 'daar heb je pietje precies weer'. Maar bovenal was er altijd een warm welkom met veel gezelligheid en ontspanning in de koffiekamer. Ik ga niet alle namen noemen, want dan ga ik zeker onterecht personen vergeten. Maar Dirk, Gerrit Jan, Gerald, Wendy, Harmen, Antonique, Johan, Patrik, Wilfred en Arnold, bedankt voor jullie vertrouwen en kundige hulp!

\section{Colleagues abroad}

The SAPHIR consortium played an important role during my whole $\mathrm{PhD}$ and gave me the opportunity to collaborate and bond with many excellent researchers and technicians from all over Europe. I would like to thank all persons involved in this project, but a few of them require special attention. Artur and Gael thank you for having me around in Bern. I 
will never forget your contribution to the first publication of this thesis. Chapter 2 was a personal mile-stone, which gave me wings. Anne and Joanne, thank you for introducing me into the world of skin vaccination and for your friendship and trust in our work with the dissolving microneedle patches. Sungwong and Ivan, you made the trips to the Royal Veterinary College in London memorable. Damer thank you for supporting this. These visits were an excellent opportunity to extend my knowledge in chicken immunology and Eimeria. Isabelle, you had the challenging task to lead this large collaboration, which created opportunities for PhD students to develop and expand their network. Thank you for your trust and personal approach.

Kerstin and Karin, thank you for the warm welcome in Copenhagen and your help with analysing the samples of Chapter 3. I am very proud that I can use our data in this thesis.

\section{Collega's WBVR}

Hetty, Astrid, Rene, Jan, Marga, Paul, Lars, Conny, Ksenia, Hélène, Helmi en Monique, bedankt voor jullie betrokkenheid en bijdrage aan dit proefschrift. Ik hoop, dat ik dat niemand in het bijzonder vergeten ben...... Uiteraard wil ik de hele IB groep en de collega's binnen en buiten gebouw 215 bedanken voor hun steun en interesse de afgelopen jaren. Wat hebben wij toch veel kennis op dit instituut! Soms was het even zoeken naar de juiste persoon, maar altijd was er wel een luisterend oor of de nodige hulp in de diverse fasen van mijn promotietraject. Bedankt WBVR!

\section{Cell Biology and Immunology group (CBI)}

It was always a pleasure to come to Wageningen and I hope that I can continue this collaboration with the group. Hilda, Edwin, Sylvia and Maria, thank you for your interest and support during my PhD. Mirelle my'porcine buddy', thank you for your friendship and discussion moments. Our trip to Seattle is still in my mind. Heidi, 'veterinair jaargenoot' and late discovery, thank you for sharing thoughts and for your critical review of the discussion.

Arifa, it was a pleasure to finalize my PhD in 'our room', a place of rest and gezelligheid.

PhD colleagues at $\mathrm{CBI}$, although I was not a constant factor in Wageningen, I always felt like part of the group. Paulina, Adria, Mojtaba, thank you for asking me around for a coffee or lunch. This were essential moments, especially in the last months of my PhD. Anja, thank you for your help with the immunohistochemistry. Ben, thank you for your help with the flow cytometry. It was a pleasure to work with both of you. Kristina, you were a special student, with many talents. Thank you for your help! 


\section{Vriendinnen}

Lieve diergeneeskunde vriendinnen, al ben ik misschien een stille, ik ben enorm blij en trots om deel uit te maken van ons groepje. Dit jaar vierden we het 20 jarige jubileum van ons jaarlijkse uitje in Gent. Ja, Claudia, Sandra, Hester, Debora, Pian en Hanneke, we worden ouder, we veranderen, maar we blijven verbonden. Bedankt voor deze bijzondere vriendschap en voor jullie hulp bij het bewandelen van het levenspad. Ik kijk uit naar de volgende 20 jaar.

Geen leven zonder tennis, maar zeker niet zonder tennisvriendinnen. Martina, Nicole, Marieke en Leanne, bedankt voor het plezier op de tennisbaan, de gezelligheid en de goede gesprekken. De afgelopen jaren was mijn deelname zeker geen $100 \%$ en zoals altijd beloof ik weer beterschap voor komend jaar. Martina, onze band gaat wat verder dan tennis. Bedankt voor het waarderen en bespreken van onze gedeelde passie voor werk en uitdagingen. Niet iedereen begrijpt deze drang. Ik soms zelf ook niet.

Lieve Marga en Linda, wat is het toch heerlijk om met jullie te eten en te borrelen. Het is altijd gezellig, ontspannen en alles is bespreekbaar. Niks is er veranderd sinds onze kennismaking ruim 20 jaar geleden in Thessaloniki. Na een avondje met z'n drietjes, ga ik altijd weer naar huis met een blij gevoel en met nuttige tips. Jullie bewonderen mij, maar ik bewonder jullie. Bedankt voor jullie onvoorwaardelijke vriendschap.

\section{Pathologie}

Het eigenlijke startschot voor de omslag naar de wetenschap werd gegeven op de afdeling pathologie in Utrecht. De opleiding tot veterinair patholoog was een bijzonder traject. De verbondenheid, ontstaan in en buiten de SIO-kamer, zal nooit verloren gaan. Daarom wil ik mijn oud-collega's graag heel hartelijk bedanken voor mijn vorming tot patholoog, wetenschapper en mens. Ik voel me nog steeds kind aan huis bij de pathologie in Utrecht.

Alan, thank you for your cheerful chats on Skype and for your help with the introduction and summary. Judith, bedankt voor je luisterend oor en advies. Erik, Ivo en Helene, bedankt voor het fijne samenzijn met wijn en spijs. Manon, bedankt voor de gezellige avondjes bij Claudia. Andrea, bedankt voor je vertrouwen. Zonder jou was dit pathologie en onderzoek avontuur nooit begonnen.

\section{Amsterdam en katten}

Lieve Els, bedankt voor jouw prachtige tekeningen, die op de kaft van dit proefschrift prijken. Ik wist het gelijk, er is maar 1 persoon die deze kaft kan ontwerpen. Ik ben trots op zo'n getalenteerde vriendin, waarmee ik een passie voor katten deel. Mijn huidige werk is uiteraard heel wat anders, dan toen we elkaar ontmoetten in de gezelschapsdieren praktijk. Ik heb altijd gewaardeerd hoe je al mijn stappen hebt gesteund. Judith, deze 
waardering geldt ook voor jou. Helaas was de tijd beperkt de afgelopen jaren, maar ik kom altijd met veel plezier naar de Poezenboot. Al pratend met jou, ga ik weer terug naar mijn wortels: dierenarts en katten liefhebber. Ook heb ik op jouw werkplek heerlijk aan de algemene discussie kunnen schrijven. Jouw stekkie gaf rust en inspiratie. Bedankt voor het delen.

\section{Sus scrofa domesticus en Felis silvestris catus}

Lieve varkentjes, zonder jullie was er geen boekje geweest. Bedankt voor jullie onbewuste bijdrage en knuffelmomenten. Nergens kon ik me beter ontspannen dan bij jullie in de stal. Onderzoeker en dierenliefhebber zijn, is een uitdagende combinatie. Als onderzoeker neem ik dan ook alle verantwoordelijkheid voor het onderzoek in mijn proefschrift en als dierenliefhebber heb ik dit naar eer en geweten uitgevoerd. Bella, wij hebben aan een blik genoeg. Je wist precies wanneer ik een kopje nodig had in dit promotietraject.

"Time spent with cats (and pigs) is never wasted."

Sigmund Freud (modified)

\section{Familie Grinwis}

Lieve schoonfamilie, bedankt voor jullie steun, betrokkenheid en liefdevolle omarming. Elke Grinwis-verjaardag/bijeenkomst is een feest van hartelijkheid en ongedwongenheid. Als 'partner van', voel ik me nooit 'koud' en er was altijd begrip voor de afwijkende agenda tijdens dit promotie-traject. Is het nu klaar? Dat antwoord mogen jullie zelf geven.

\section{Familie Vreman}

Lieve Remco, wat ben ik enorm trots op hoe jij de bakkerij van onze ouders voortzet. Een ambacht goed beheersen is net zo waardevol als verschillende 'afkortingen' rond je naam, vergeet dit nooit! Je bent een broertje om op te bouwen en om lief te hebben. Ik reken erop om met jou de langste liefdevolle relatie uit mijn leven te hebben. Ik kom graag weer helpen in de bakkerij met de feestdagen. Amber, Ben en Nathalie, 'Tante Poes' zijn is voor mij belangrijk en ik beloof dat ik na dit proefschrift meer tijd voor jullie ga vrijmaken.

Lieve ma en pa, bedankt voor jullie levenslange steun en liefde. Jullie hebben altijd achter mij gestaan, ook al was ik soms moeilijk te doorgronden. In de afgelopen jaren was het soms lastig te bevatten wat ik nu precies uitspookte in Lelystad. Maar altijd reageerden jullie vol trots op nieuwe resultaten of op een publicatie. Ook kwamen er steeds meer vragen, waaraan ik merkte dat jullie begrepen dat een dierenarts ook onderzoek kan doen. Nu dit boekje klaar is, wil ik graag zeggen, dat ik enorm trots ben op mijn lieve ouders. Ik hou van jullie.

Lieve ma, ik zie steeds meer overeenkomsten tussen ons en ik hoop dat we Ootmarsum nog vaak samen kunnen bezoeken. Lieve pa, werk kan een passie zijn waarmee je 
eindeloos kunt doorgaan. Soms mopperen we over uw dagelijkse werkzaamheden in de bakkerij. Als 75-jarige mag er meer rust zijn of misschien een hobby? Ik bewonder deze passie voor de bakkerij en ik weet dat u dit niet kunt en mag loslaten. Hopelijk kan ik ook nog achter de microscoop zitten na mijn pensioen, $u$ bent mijn voorbeeld.

"If you have passion, there is no need for excuses because your enthusiasm will trump any negative reasoning you might come up with. Enthusiasm makes excuses a nonissue"

Wayne Dyer

"Pleasure in the job puts perfection in the work"-

Aristotle

Lieve Guy, jij bent mijn grootste passie. Je bent een bijzondere pallieter, wars van pretenties, slim, meestal nuchter en soms een tikkeltje eigenwijs. Bij jou kan een volledig mezelf zijn en samen vormen we een 'pittig stel'. Bedankt voor je liefde, zorgzaamheid, steun en talrijke vorstelijke malen. Na dit proefschrift komt de kidde uit het keukenkastje en de pastamachine uit zijn doos. 
This work is part of an European collaboration called SAPHIR (Strengthening Animal Production and Health through the Immune Response) https://cordis.europa.eu/project/id/633184), which has received funding from the European Union's Horizon 2020 Programme for research, technological development and demonstration under the Grant Agreement $n^{\circ} 633184$. This publication reflects the views only of the author, and not the European Commission (EC). The EC is not liable for any use that may be made of the information contained herein.

Financial support from Wageningen University for printing this thesis is gratefully acknowledged.

Cover design: "Miss Piggy" by Els Scholten

Layout: Dennis Hendriks / ProefschriftMaken.nl

Printed: $\quad$ ProefschriftMaken on FSC-certified paper 


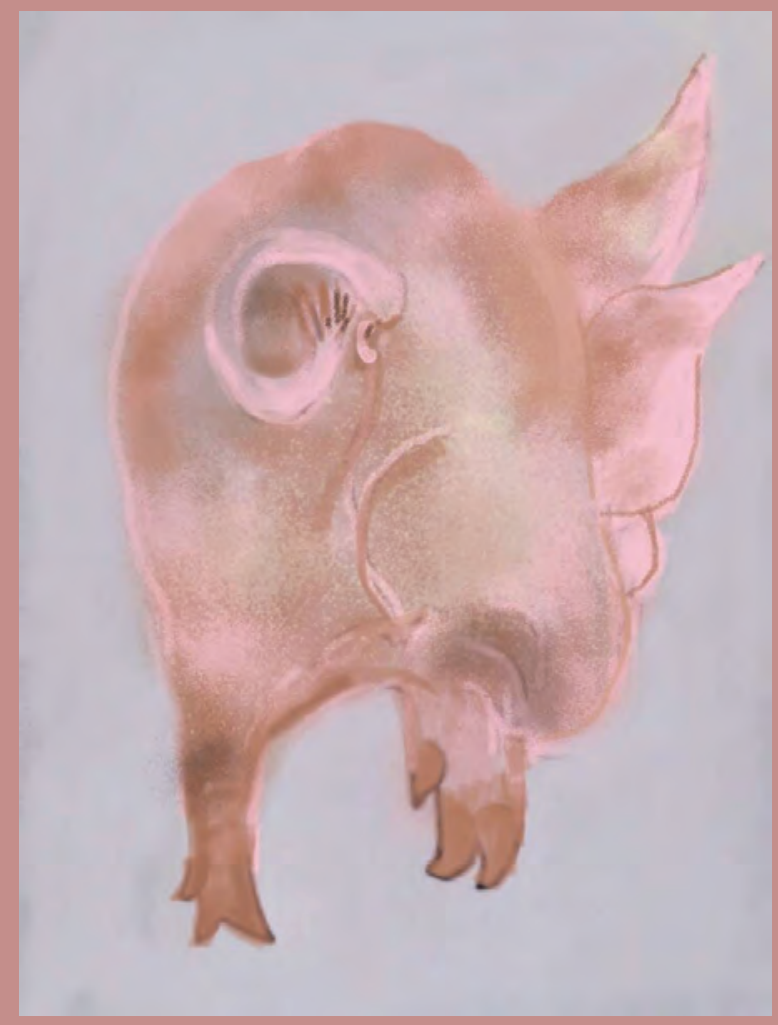

